NEW MEMBRANE STRATEGIES FOR IMPROVED ARTIFICIAL KIDNEY DEVICES

Ilaria Geremia 



\title{
NEW MEMBRANE STRATEGIES FOR IMPROVED ARTIFICIAL KIDNEY DEVICES
}

\author{
DISSERTATION
}

to obtain

the degree of doctor at the Universiteit Twente,

on the authority of the rector magnificus,

Prof. dr. ir. A. Veldkamp,

on account of the decision of the Doctorate Board

to be publicly defended

on Friday 22 January 2021 at 14.45 hours

by

\section{Ilaria Geremia}

born on the 6th of November, 1988

in San Vito al Tagliamento, Italy 
This dissertation has been approved by:

Supervisor

Prof. dr. D. Stamatialis

Cover design: Ilaria Geremia and Simone Razzano

Printed by: Gildeprint

Lay-out: Ilaria Geremia

ISBN: 978-90-365-5104-5

DOI: $10.3990 / 1.9789036551045$

(C) 2020 Ilaria Geremia, The Netherlands. All rights reserved. No parts of this thesis may be reproduced, stored in a retrieval system or transmitted in any form or by any means without permission of the author. Alle rechten voorbehouden. Niets uit deze uitgave mag worden vermenigvuldigd, in enige vorm of op enige wijze, zonder voorafgaande schriftelijke toestemming van de auteur. 


\section{Graduation Committee:}

Chair / secretary:

Supervisor:

Committee Members:
Prof. dr. J.L. Herek (University of Twente)

Prof. dr. D. Stamatialis (University of Twente)

Prof. dr. D. Stamatialis (University of Twente)

Prof. dr. D.W. Grijpma (University of Twente)

Dr. ir. W.M. de Vos (University of Twente)

Dr. K.G.F. Gerritsen (UMC Utrecht)

Prof. dr. J.P. Kooman (UMC Maastricht)

Prof. dr. J. Van Der Vlag (Radboud University)

Prof. dr. G. Catapano (University of Calabria) 



\section{Table of contents}

Chapter 1

General Introduction

Chapter 2

In vitro assessment of mixed matrix hemodialysis membrane for achieving endotoxin-free dialysate combined with high removal of uremic toxins from human plasma

Chapter 3

Ex vivo evaluation of the blood compatibility of mixed matrix hemodialysis membranes

Chapter 4

Development of hollow fiber hemodialysis membranes for outside-in filtration

\section{Chapter 5}

New mixed matrix membrane for the removal of urea from dialysate solution

Chapter 6

Development of positively charged nanofiltration hollow fiber membranes for ammonium removal

Chapter 7

Conclusions and Outlook

Summary

Nederlandse samenvatting

List of abbreviations

About the author

List of publications 



\section{Chapter 1}

\section{General Introduction}

Parts of this chapter have been published in:

O. Ter Beek, I. Geremia, D. Pavlenko, D. Stamatialis, Chapter 3: “Advanced Blood Purification Therapies", book "Biomedical membranes and (bio)artificial organs", World Scientific, 2017, 59-82.

I. Geremia and D. Stamatialis, Innovations in dialysis membranes for improved kidney replacement therapy, Nature Reviews Nephrology, 2020, 16, 550-551 


\section{The kidneys and kidney failure}

The kidneys, located in the abdominal cavity, regulate many body functions, as the excretion of metabolic products and exogenous substances, body fluid osmolarity and volume, electrolyte and acid-base balance and secretion of hormones [1]. The nephrons (approximately $1.8-2$ millions in healthy people) are the functional units of the kidney and are responsible for blood purification and urine formation [1]. In the nephron (Figure 1), blood initially passes through the glomerulus, a network of blood capillaries, where filtration of small and middle-size molecules and removal of excess fluid occur. The resulting filtrate is then transferred to the proximal tubules, which are responsible for the reabsorption of essential components and for additional removal of a wide range of toxins, including protein-bound toxins (PBTs). Removal of PBTs by the natural kidneys is an efficient, complex and sophisticated excretion process which, unfortunately, current dialysis membranes cannot reproduce. As a matter of fact, in order to allow the excretion of these PBTs with the urine, a range of transporters in the proximal tubules cooperate to shift the binding of PBTs from proteins and to allow their excretion in basolateral uptake and luminal excretion [2].

In addition, the kidneys have critical endocrine function by producing erythropoietin, vitamin D, angiotensin, prostaglandins, and other endocrine compounds [3]. Healthy people have, in general, 2 kidneys but one functioning kidney is usually enough to provide all essential kidney functions to the human body. This brief paragraph on kidney functions is meant to be only an introduction to the next sections and further details on kidney physiology are beyond the objectives of this thesis. 


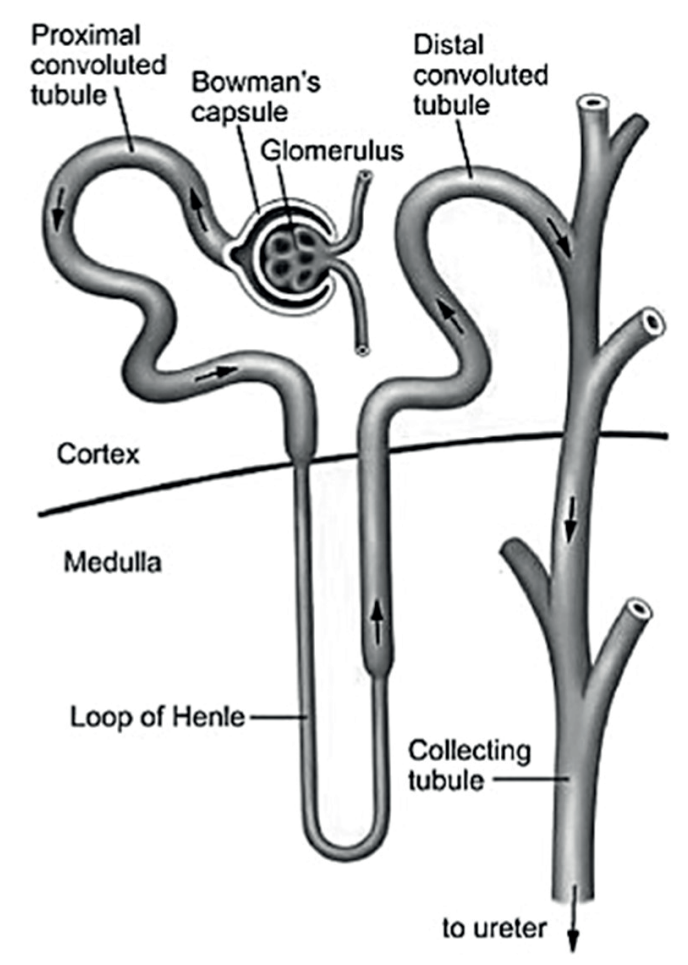

Figure 1.Schematic representation of the nephron, adapted from [4].

Many factors can cause kidney failure; among these are diabetes, hypertension, the use of medicines, as well as an unhealthy lifestyle (for example, smoking, overweight, salty diets) [5]. As a result of kidney failure, accumulation of waste products and fluids in the body occur.

Five stages have been identified for chronic kidney disease, from very mild damage, in stage 1 , to complete renal failure, in stage 5. When the GFR (Glomerular Filtration Rate) is consistently below $15 \mathrm{~mL} / \mathrm{min}$ per $1.73 \mathrm{~m}^{2}$, the patient suffers of End Stage Kidney Disease (ESKD) and has to undergo renal replacement therapies (RRT) [6]. Kidney transplantation is the best solution for patients in need of RRT. However, patients not suitable or while waiting for kidney transplantation have to undergo dialysis, either peritoneal or hemodialysis (HD) therapy. Recently, it has been estimated that 3 million people undergo HD worldwide [7]. However, given the increasing incidence of cardiovascular disease and diabetes, the number of ESKD patients is expected to rise during the next years both in industrialized and developing countries and reach 5.4 million by 2030 worldwide [5]. 


\section{Extracorporeal blood purification therapies}

\subsection{Uremic toxins}

The EUTox work group of the European Society for Artificial Organs (ESAO) divided all blood toxins into three main categories: 1) water-soluble toxins, with molecular weight (MW) smaller than $500 \mathrm{Da}$ (as creatinine and urea), 2) middle molecules, with MW larger than $500 \mathrm{Da}$ (as $\beta 2$ microglobulin, parathyroid hormone, peptides and small proteins) and 3) protein-bound uremic toxins (PBTs) (as indoxyl sulfate, hippuric acid and p-cresyl sulphate) [8].

With current therapies, the removal of small water-soluble uremic toxins from the blood is higher compared to the middle molecules and PBTs since, due to their low MW, they can easily permeate through the HD membranes. Unfortunately, the inability of current membranes to remove middle and large-sized uremic toxins and PBTs has major repercussions for patient outcomes [9].

The ability to achieve adequate clearance of middle-sized and large uremic toxins without loss of albumin requires membranes to have a large pore size and narrow pore distribution, with a molecular weight cut-off (MWCO) close to, but lower, than that of albumin $(\sim 66 \mathrm{kDa})$, thus mimicking the transport properties of the kidney glomerular filtration barrier [10]. An important middle-size uremic toxin is $\beta 2$-microglobulin, elevated concentrations of which have been associated to amyloidosis, increased morbidity and mortality of ESKD patients $[11,12]$. The removal of PBTs is still challenging due to their binding to blood proteins (especially albumin) and the fact that current HD therapies are designed to retain proteins in the blood (their MWCO is lower than $66 \mathrm{KDa}$ which is the MW of albumin). PBTs are associated to cardiovascular disease, generation of reactive oxygen species and progression on chronic kidney disease [13]. In order to improve their clearance, strategies which can maintain a high toxin concentration gradient between the blood and dialysate compartment have been proposed. Amongst these, there is the mixed matrix membrane (MMM) strategy developed earlier by Stamatialis et al with small activated carbon (AC) embedded in the polymer matrix [14- 
16] and the use of binding competitors of albumin, as for example not esterified fatty acids and tryptophan [9].

\subsection{Hemodialysis (HD)}

In HD a semi-permeable membrane is responsible for the transfer of accumulated uremic toxins from patient's blood to the dialysate. The dialysate, also called dialysis fluid, is a pure water solution containing electrolytes, most commonly sodium, potassium, calcium, magnesium, chloride and bicarbonate, and glucose or dextrose. In this thesis with the term "dialysate" we will refer to the dialysis water solution before HD therapy, while with the term "spent dialysate" we will refer to the dialysate after HD treatment and, therefore, containing the uremic toxins transported from the patient's blood.

In HD, mainly small MW water-soluble toxins diffuse through the artificial HD membranes, thanks to the osmolar gradient between blood and the dialysate while larger molecules, PBTs, platelets and blood cells are retained in the blood. In fact, as already mentioned above, HD membranes have a MWCO lower than $66 \mathrm{KDa}$, thus being able to reject albumin. Since the Kolff's rotating drum kidney introduced in 1945, HD has come a long way [17]. It has dramatically evolved in the 1960s thanks to the placement on the market of the first hollow fiber (HF) membranes, which guaranteed improved blood rheology and, thanks to the high surface area, enhanced solute mass transfer [17]. The poorly hemocompatible regenerated cellulose membranes were soon replaced by modified cellulose and nowadays the majority of the market is dominated by synthetic membranes, comprising polyethersulfone (PES) or polysulfone (PSu) polymers [18]. However, outstanding blood compatibility issues are still pending, as the adsorption of blood proteins (due to the hydrophobicity of polymers used for the fabrication of HD membranes, as PES and PSu) on the membrane leading to thrombosis [19]. In order to limit this last complication, the blending of hydrophilic additives (such as polyvinylpyrrolidone (PVP) and polyethylene glycol) to the membrane forming polymer has 
significantly helped during the last years [17]. Nevertheless, the hydrophilic additives can leak from the membranes, thus decreasing the hemocompatibility profile of the membrane over time [19].

HD is mainly performed in the clinics 4 hours - 3 times per week. This intermittence typical of HD treatment does not mimic natural kidney filtration and leads to the accumulation of toxins and of excess fluid between treatments. Several studies have shown that prolonged HD sessions would improve the removal of toxins from the blood of ESKD patients and may thereby increase patients' overall health and quality of life [20-24].

\subsection{Hemofiltration $(\mathrm{HeF})$ and hemodiafiltration (HDF)}

Beyond HD, membranes for convective therapies such as hemofiltration $(\mathrm{HeF})$ and hemodiafiltration (HDF) have also been introduced.

$\mathrm{HeF}$ is based on the convection principle; the transport of fluid and solutes across a semi-permeable membrane occurs due to transmembrane pressure difference allowing the transport of larger solutes through the membrane [18]. HDF combines diffusion with convective transport (Figure 2). 


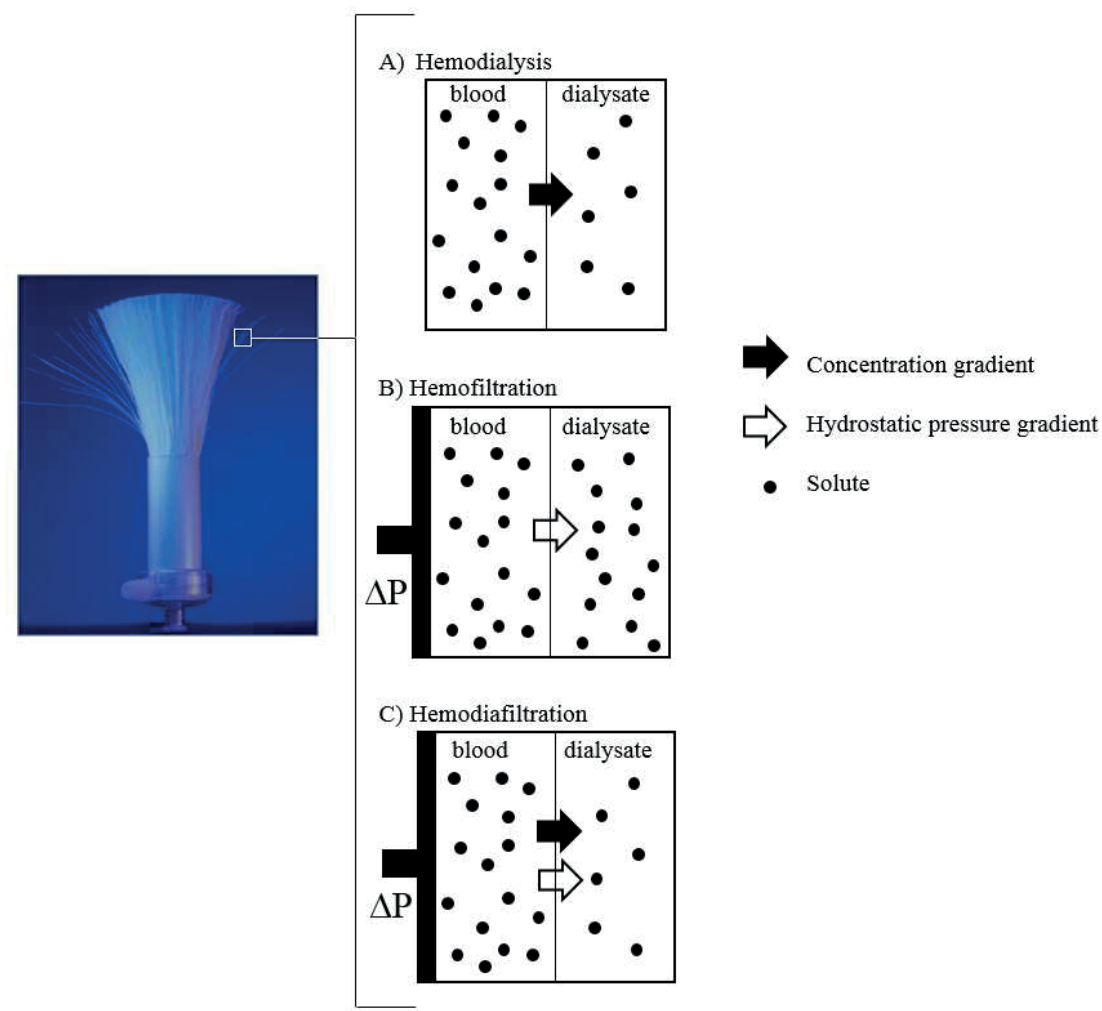

Figure 2. Schemes of the mechanisms of hemodialysis, hemofiltration and hemodiafiltration. The image is taken from [25].

In HDF, the combination of diffusion and convection leads to an increase of the filtration rate which enhances the removal of water-soluble solutes compared to what can be obtained with diffusion alone; this is reflected in an improvement of the performance of the HDF in terms of morbidity and mortality [18]. In HeF and HDF the fluid removal exceeds that gained by the patient, contrary to HD where the fluid removal is equivalent to the fluid gained by the patient between the treatments. Thus, in $\mathrm{HeF}$ and HDF a suitable substitution liquid has to be infused to the patient [18]. The electrolyte replacement solution can be delivered to the extracorporeal blood stream before the filtration process ("pre-dilution HDF"), during the filtration process ("mid-dilution HDF") or downstream from the hemofilter (“post-dilution HDF”). 


\subsection{Sorption based therapies}

Hemoperfusion (HP) was introduced for the first time by Yatzidis in early 1960s [26]. He described a new extracorporeal apparatus for the removal of toxins (as, for example, creatinine, uric acid, phenolic compounds, guanidine bases) directly from the recirculating heparinized blood of patients with ESKD by perfusion over charcoal [26]. Nowadays the number of HP therapies is exponentially increasing, not only for kidney failure but also in the treatment of other disease states, such as liver failure, sepsis, cardiopulmonary bypass, drug overdoses and multi organs failure [27]. HP, in contrast to the HD treatment, seems to be more efficient for the removal of middle molecules, cytokines and PBTs from patients' blood. However, the inability to correct the fluid balance, to remove urea and frequent hemocompatibility complications noticeably lowered its use [28].

Adsorption is solute specific rather than size-specific and the binding depends on the chemical affinity between the solute and the sorbent and on the accessible surface area of the sorbent for the adsorbate which determines the availability of binding sites. Moreover, to have effective adsorption, various other parameters have to be taken into account including pressure drop, resistance to sterilization and hemocompatibility (when the sorbent have to be in direct contact with the blood). Sorbents can be developed in different formulations (particles, resins and membranes) but the most common forms are beads and micro-particles which combine a large surface area with high porosity.

Sorbents used in HP can be natural or synthetic. Among the natural sorbents, AC is the most commonly used. It is a broad-spectrum sorbent which can remove different kinds of substances, such as organic metabolic wastes, drugs and other undesirable components from the blood. Although AC can adsorb many of the uremic toxins in the middle molecule weight range, it does not remove urea or acidic solutes. Because of this, when the blood of patients needs to be purified, HP and HD need to be combined. Despite its great adsorption capacity, AC has rather poor biocompatibility due to its high affinity to blood components, such as platelets and blood proteins. For this reason, the direct contact of AC with blood in a HP circuit should be avoided. The hemocompatibility of different kinds 
of sorbents can be improved by the addition of hemocompatible coatings or by chemical modifications of the starting materials $[29,30]$.

The great success of HP has also led to a growing interest towards new sorbents formulations. Many efforts have been and are still being focused on developing smart sorbents able to bind one or more desired target molecules. Not only the chemistry of the sorbents can be tuned, but also their porosity can be engineered.

\section{New concepts in extracorporeal blood purification therapies}

\subsection{Mixed Matrix Membrane (MMM)}

Recently, a MMM has been proposed for the removal of a range of uremic toxins, including PBTs [14-16, 31, 32]. These membranes consist of two layers: a polymeric porous layer with embedded AC (the mixed matrix membrane layer) and a porous polymeric particle-free layer (Figure 3).

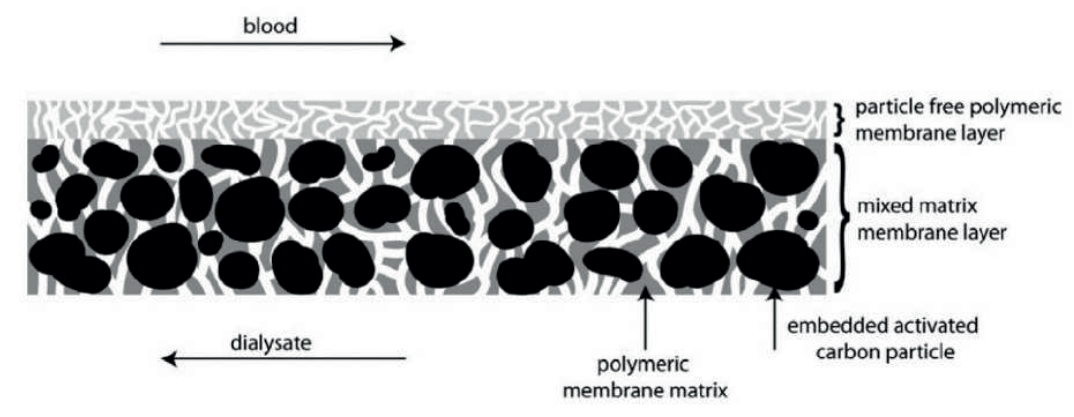

Figure 3. Concept of MMM which combines filtration and adsorption in one step. The image is taken from [25].

The incorporation of AC particles into the macroporous MMM enables the combination of filtration and adsorption in one membrane. The particle-free polymeric layer prevents direct contact between patient's blood and the AC and it is responsible for the selectivity of the whole membrane. The MMM poses a number of advantages compared to conventional therapies. Firstly, the use of relatively small adsorptive particles increases the available surface area for the adsorption of the uremic toxins without high pressure drop which is very often observed in bed adsorption column. Secondly, the use 
of adsorptive particles inside the outer layer of the membrane increases the removal of the toxins by keeping the concentration gradient of the toxins at the maximum level, as most of the toxins that reach the outer layer are adsorbed. The additive effect of the adsorption inside the membrane has been recently demonstrated for the removal of the PBTs in comparison to commercially available HD membranes in earlier studies $[15,16,31,32]$.

\subsection{Long-term / continuous treatment}

Current forms of RRT remove uremic toxins in an intermittent manner, typically for $4 \mathrm{~h}$ three times per week. Longer and/or continuous RRT (for example, with use of portable artificial kidney (PAK) and wearable artificial kidney (WAK) devices) would more closely mimic the continuous nature of kidney filtration and likely improve patient outcomes as well as enable greater patient mobility and quality of life $[23,24]$. Besides higher toxin removal, the longer operational time may lead to additional advantages, including a reduction of filter replacement costs, lower anti-coagulation requirements, lower blood loss, significant reduction of nursing time, lower disturbances in patient blood pressure and reduction of the likelihood of infection [20-22].

Challenges for membrane dialyzers in the context of continuous therapy are the need for long-term blood compatibility (owing to the prolonged contact time of the patient's blood with the membranes) and issues relating to blood clotting, which would limit the lifetime of the device. Despite significant advances on improving membrane hemocompatibility over the last decades, the maximum fiber life is typically in the range of 15 - 40 hours even with the use of complex citrate anti-coagulation regimes [33]. Most currently available synthetic membranes are composed of hydrophobic polymer blends, such as PES or PSu, with hydrophilic additives such as PVP. However, these additives can elute during sterilization processes or during filtration [19]. The elution of these additives can potentially be prevented by grafting or coating various hydrophilic additives to the membrane lumen surface [19]. Recently, new materials for preparation of membranes have been proposed. For example, Ter 
Beek et al. described a new hollow fiber PES-Slipskin (SS) polymer blend membrane for long-term filtration. SS is a random copolymer that consists of a hydrophilic block (N-vinylpyrrolidone) and a hydrophobic block (N-butylmethacrylate) [19]. It has been shown that PES-SS blend membranes have high fouling resistance, good hemocompatibility and high removal of uremic toxins, suggesting that these membranes could be suitable for prolonged or continuous RRT modalities [19]. Various approaches have also been suggested to minimize blood clotting inside the dialyzer. One interesting approach is the use of outside-in filtration (OIF) (which will be described in more detail in section $3.3)$.

Finally, continuous RRT requires prolonged circulation of the dialysate. This prolonged circulation increases the risk that bacterial pyrogens present within a contaminated dialysate might reach the patient's blood, with potential adverse consequences. It is therefore important that the dialysis membrane also acts as barrier to protect the patient's blood by blocking the transport of bacterial pyrogens [31].

\subsection{Outside-in filtration}

As described earlier, in standard HD the blood flows in the lumen of hollow fiber hemodialyzer, while the dialysate into the inter-fiber space (IFS), in the so-called "inside-out filtration" mode. In this standard configuration thrombi can be deposited and blood clots can be formed at the inlet to the fiber, blocking the blood flow through the fiber and consequently lowering blood clearance and filter life [34]. When many fibers in the filter become clotted, the filter has to be replaced. In order to prevent fiber clotting, in 1992 Catapano et al. discussed the concept of "outside-in filtration" mode (OIF) for blood oxygenators [34]. In the OIF mode the blood flows into the IFS while the dialysate flows into the intraluminal space. Thus, thrombi that are deposited in the IFS will have only a minimal effect on membrane function because blood can flow around and bypass them without any marked 
reduction in membrane surface area [34]. Recently, the concept of OIF has been applied to HD by Dukhin et al. [33], who showed that commercial dialyzers, actually designed for standard inside-out filtration, could operate for more than 100 hours when applied in the OIF mode. In fact, in the OIF mode, thrombi will deposit in the IFS at the blood entrance, having only little disturbance on the blood flow and filtrate flux due to the three-dimensional system of interconnected hydrodynamic flow channels in the IFS [33]. For broader implementation of OIF new membranes that have the hemocompatible selective layer on the outer fiber surface are required.

\subsection{Wearable Artificial Kidney (WAK)}

The WAK or PAK devices could prolong the dialysis time thus eliminating the problem of having intermittent dialysis treatments. This could improve the quality of patients' life as well as reduce hospital length stay and care unit utilization [35]. The development of a WAK is really challenging since it needs to satisfy a lot of different technical and clinical aspects [35], listed below:

- proper vascular access (easy to connect and disconnect) in order to have a continuous therapy and to reduce as much as possible the risk of infections and clotting;

- Adequate safety measures in the circuit including air detection, pressure sensors, visual and audible alarms;

- Remote control to allow the patient to accurately program and deliver the scheduled therapy together with a regular control of the physiological state of the patient;

- Independence from the electrical outlet;

- Minimal volume of dialysate (preferably $0.5 \mathrm{~L}$ ) that must be continuously regenerated;

- Lightweight and ergonomic design that would be adapted to the body.

The first idea of WAK for HD has been reported in the 1970s by the team of Kolff [36, 37]. Recently new research has been published showing interesting and promising results. Some of them use 
extracorporeal HD [38] while others use peritoneal dialysis as a treatment modality (for example, ViWAK and AWAK) $[39,40]$. One of the challenges for the implementation of the WAK is the need to dramatically reduce the amount of dialysate and to be independent on a fixed water supply.

The WAK devices have the potential to become a real practice for the treatment of chronic kidney patients. Although at the moment larger clinical trials are needed to confirm their safety and efficacy.

\section{Dialysate regeneration}

\subsection{Pyrogenic contamination of the dialysate}

The production of safe pyrogen-free dialysate for HD requires multi-step water purification treatments, being therefore very water demanding (nearly $500 \mathrm{~L}$ of tap water, with two third discharged to drain), costly and energy consuming [41-43]. However, even when ultra-pure water is obtained, microbiological biofilms may form in the HD machine and/or water purification system due to inadequate design of the tubing system and improper machine maintenance [41, 44-46]. Importantly, water scarcity and inadequate water purification facilities worsen contamination risk in developing countries [47].

As a consequence of bacterial growth in the dialysis machine and/or water purification systems, bacterial pyrogens could be released and transferred into the patients' blood, leading to adverse effects, including cardiovascular side effects, such as inflammatory amyloidosis, atherosclerosis, hypotension, but also fever, headache and muscular cramps [43-46, 48-50]. In fact, the prolonged circulation of the dialysate, required by continuous RRT worsen the risk that bacterial pyrogens might reach the patients' blood. It is therefore important that the dialysis membrane also acts as barrier to protect the patients' blood by blocking the transport of bacterial pyrogens.

Several studies in the literature have investigated the ability of commercial hemodialyzers to avoid back-transport of bacterial disintegration products from the dialysate to the blood. For example, it has 
been observed that a high grade of dialysate contamination caused breakthrough of pyrogens into the blood with high-flux DIAPES membrane (DIAPES HF800, Bellco) and polysulfone membrane (BLS 627, Bellco) [51]. Weber et al. [52] also reported significant endotoxin transfer to the blood compartment for both low- and high-flux DIAPES (Bellco) membranes, while Helixone (Fresenius) presented the highest endotoxin adsorption. Schepers et al. [50] compared the permeability of pyrogens using membranes with comparable composition but different pore-size and reported low but detectable amount of endotoxins in the blood compartment with more open membranes.

\subsection{Dialysate regeneration for Wearable Artificial Kidney (WAK) devices}

In the case of the WAK, the use of a reduced volume of dialysate (preferably $0.5 \mathrm{~L}$ ) to be continuously regenerated within the system will result in light-weight and miniaturization, essential for portability and wearability. In order to regenerate the spent dialysate, ions (as for example phosphate and potassium), small organic waste solutes (as for example creatinine and urea) and middle molecules (as $\beta 2$-microglobulin) need to be removed [53]. Ions can be removed by means of ion-exchangers, while most organic waste solutes can be efficiently removed by AC [53]. However, the biggest challenge for dialysate regeneration is the removal of urea. Strategies proposed for urea removal are discussed in the next section.

\subsection{Urea removal from dialysate}

Urea is the main waste product of nitrogen metabolism. Although urea can be easily removed by the patients' blood by means of HD, its removal from spent dialysate is very challenging due to its small molecular weight (MW $=60 \mathrm{~g} / \mathrm{mol}$ ) and its chemical similarity to water [53]. It is very important to include in PAK and WAK devices dialysate regeneration systems able to remove urea due to the fact that high plasmatic urea concentrations are harmful. High urea concentration in the dialysate can, in 
fact, lead to insulin resistance, disruption of the gastrointestinal barrier and production of radical oxygen species, amongst others [54].

Many strategies have been proposed for the removal of urea from spent dialysate. Among them are the enzymatic decomposition of urea by means of ureases (applied in the REDY device) [53, 55], electrooxidation of urea, urea-molecular imprinted polymers [53, 56-58], as well as many urea sorbents. The latter can bind urea via physisorption (hydrogen bonding, van der Waals and dipole interactions), as for activated carbon [59-61], silica [62], zeolites [63] and MXenes nanosheets [64]. Others, such as chitosan complexed with metal ions, can bind urea via coordination bonding [65-68]. Furthermore, carbonyl-type sorbents (such as aldehydes, $\alpha$-ketoaldehyde hydrates, ninhydrins, $\alpha$ ketoesters and glyoxaldehydes) can covalently bound urea [53].

Unfortunately, due to the in some cases formation of toxic byproducts, high cost or because of their inefficiency, none of the proposed strategies have found yet clinical implementation. 


\section{Scope of the thesis}

Despite recent advances in HD membranes, current HD therapies are unable to replace kidney function. They provide limited removal of uremic toxins (especially PBTs) and they do not function continuously. Moreover, on the way towards the development of WAK and PAK systems, efficient and affordable strategies for the removal of urea from spent dialysate still have to be developed. Therefore, due to the inability to regenerate and recirculate spent dialysate, current HD therapies still depend on a fixed water supply thus requiring an enormous amount of ultra-pure water and limiting mobility of dialysis patients. Furthermore, the risk of bacterial contamination of the dialysate and of the HD machine still persists in the clinics with potential adverse consequences for patients' health. The scope of this thesis is to develop new membrane-based strategies for improved artificial kidney devices. More specific, we aim to the develop new membranes which could provide better and more continuous blood purification therapies as well as membranes which could facilitate the dialysate regeneration.

In order to achieve better and safer blood purification therapies, we investigate:

1. the implementation of a MMM composed of AC embedded in a PES/PVP matrix to simultaneously purify human blood plasma and remove pyrogens from the dialysate.

2. the blood compatibility of the MMM following ISO 10993-4, which is a prerequisite for its implementation in the clinic.

3. the development of HF for OIF to facilitate long term blood filtration, by reducing the risk of fiber clotting by blood thrombi.

In order to achieve efficient strategies for dialysate regeneration, we investigate:

1. the development of a new MMM based on PES/PVP with polystyrene-ninhydrin (PS-Nin) particles. We hypothesize that the incorporation of small PS-Nin particles in the MMM could achieve faster and better urea binding compared to the same particles in suspension. 
2. the development of a new positively-charged nanofiltration HF membrane by means of the "Chemistry in the spinneret" technology for the retention of ammonium ions derived by the hydrolysis of urea by means of ureases.

\section{Outline of the thesis}

Chapter 2 describes the application of a dual layer MMM for achieving high removal of endotoxins from dialysate and preventing transfer of endotoxins to the blood compartment while at the same time achieving high removal of uremic toxins from human plasma. This MMM is composed of AC embedded in a PES/PVP polymer matrix [16]. We investigate the adsorption in vitro of lipopolysaccharide (LPS) on the MMM in both static and dynamic conditions. Dynamic adsorption of LPS on the MMM is also investigated in vitro in presence of PBTs in human plasma to study whether the adsorption of PBTs on the AC would compromise the simultaneous removal of endotoxins from the dialysate and vice versa. Diffusion experiments using dialysate solution contaminated with bacterial culture filtrates are also performed to assess the ability of the MMM to act as a safety-barrier to avoid transfer of pyrogens to the plasma.

In Chapter 3 we present a detailed study of the blood compatibility of the MMM to facilitate its clinical implementation. We perform ex vivo experiments, using freshly donated human blood, following the norm ISO 10993-4, as well as characterization of the membrane surface. We investigate two types of MMM, one low-flux (also studied in Chapter 2) and one high-flux membrane and the results are compared to those of a single layer HF based on PES/PVP (home-made) and to two dialyzers, Polysulfone ${ }^{\circledR}$ F60 and Cuprophan ${ }^{\circledR}$ F1.

In Chapter 4 we investigate the development of HF for OIF mode based on PES/PVP. We optimize the hollow fibers fabrication by tuning several spinning parameters to produce thin HF with the selective layer at the outer surface suitable for application in HD. The produced fibers are 
characterized in terms of morphology, surface chemistry, mechanical properties and transport characteristics. Fresenius F8HPS commercial HF is used as control for comparison.

In Chapter 5 we study the application of a new MMM HF for urea removal from dialysate solution. This MMM consists of a PS-Nin carbonyl-type sorbent embedded within a PES/PVP polymer matrix. PS-Nin particles have already been shown to be a good potential candidate for the removal of urea $[69,70]$. Here, we hypothesize that the optimal dispersion of small size PS-Nin sorbent particles within the MMM would minimize particle aggregation and lead to high particle accessibility and therefore high urea removal. The MMM hollow fiber is produced via dry-wet spinning technique. The effects of sorbent particle size, temperature and incorporation in the polymer matrix on urea removal are studied in static and dynamic experiments.

Chapter 6 describes fabrication of positively charged nanofiltration (PCN) HF membranes for removing ammonium ions. These ions can be generated by the hydrolysis of urea by means of urease. The fibers are synthesized by implementation of the "Chemistry in the spinneret" which achieves crosslinking of polyetherimide membrane forming polymer with the positively charged polyethyleneimine. Various spinning parameters are tailored to obtain membranes with high ammonium ions retention from aqueous solution.

In Chapter 7 we report the main conclusions of the thesis and discuss potential directions for future developments for improved blood purification membrane strategies as well as dialysate regeneration. 


\section{References}

[1] B. Koeppen, B. Stanton, Berne and Levy Physiology e-book, Elsevier, 2017.

[2] R. Masereeuw, H.A. Mutsaers, T. Toyohara, T. Abe, S. Jhawar, D.H. Sweet, J. Lowenstein, The kidney and uremic toxin removal: glomerulus or tubule? Seminars in Nephrology, 34(2) (2014) 191-208.

[3] M.C. Annesini, L. Marrelli, V. Piemonte, L. Turchetti, Artificial Organ Engineering, Springer, 2017.

[4] C.F. Matta, L. Massa, 1 - Information theory and the thermodynamic efficiency of biological sorting systems: case studies of the kidney and of mitochondrial ATP-Synthase, in: D. Bagchi (Ed.), Sustained energy for enhanced human functions and activity, Academic Press, 2017, 3-29.

[5] T. Liyanage, T. Ninomiya, V. Jha, B. Neal, H.M. Patrice, I. Okpechi, M.-h. Zhao, J. Lv, A.X. Garg, J. Knight, A. Rodgers, M. Gallagher, S. Kotwal, A. Cass, V. Perkovic, Worldwide access to treatment for endstage kidney disease: a systematic review, The Lancet 385(9981) (2015) 1975-1982.

[6] A. Ortiz, A. Covic, D. Fliser, D. Fouque, D. Goldsmith, M. Kanbay, F. Mallamaci, Z.A. Massy, P. Rossignol, R. Vanholder, A. Wiecek, C. Zoccali, G.M. London, Epidemiology, contributors to, and clinical trials of mortality risk in chronic kidney failure, The Lancet 383(9931) (2014) 1831-1843.

[7] L.A. Tomlinson, C.M. Clase, Sex and the incidence and prevalence of kidney disease, Clinical Journal of the American Society of Nephrology 14(11) (2019) 1557-1559.

[8] S. Eloot, W. Van Biesen, R. Vanholder, A sad but forgotten truth: the story of slow-moving solutes in fast hemodialysis, seminars in dialysis 25(5) (2012) 505-509.

[9] N. Florens, D. Yi, L. Juillard, C.O. Soulage, Using binding competitors of albumin to promote the removal of protein-bound uremic toxins in hemodialysis: Hope or pipe dream?, Biochimie 144 (2018) 1-8.

[10] M. Storr, R.A. Ward, Membrane innovation: closer to native kidneys, Nephrology Dialysis Transplantation 33 (2018) 22-27.

[11] A.K. Cheung, M.V. Rocco, G. Yan, J.K. Leypoldt, N.W. Levin, T. Greene, L. Agodoa, J. Bailey, G.J. Beck, W. Clark, A.S. Levey, D.B. Ornt, G. Schulman, S. Schwab, B. Teehan, G. Eknoyan, Serum Beta 2 Microglobulin levels predict mortality in dialysis patients: results of the HEMO study, Journal of the American Society of Nephrology 17(2) (2006) 546-555.

[12] T. Miyata, O. Oda, R. Inagi, Y. Iida, N. Araki, N. Yamada, S. Horiuchi, N. Taniguchi, K. Maeda, T. Kinoshita, Beta 2 - Microglobulin modified with advanced glycation end products is a major component of hemodialysis-associated amyloidosis, Journal of Clinical Investigation 92(3) (1993) 1243-1252. 
[13] C.J. Lin, C.F. Pan, H.L. Liu, C.K. Chuang, T. Jayakumar, T.J. Wang, H.H. Chen, C.J. Wu, The role of protein-bound uremic toxins on peripheral artery disease and vascular access failure in patients on hemodialysis, Atherosclerosis 225(1) (2012) 173-179.

[14] M.S.L. Tijink, M. Wester, J. Sun, A. Saris, L.A.M. Bolhuis-Versteeg, S. Saiful, J.A. Joles, Z. Borneman, M. Wessling, D.F. Stamatialis, A novel approach for blood purification: Mixed-matrix membranes combining diffusion and adsorption in one step, Acta Biomaterialia 8(6) (2012) 2279-2287.

[15] M.S.L. Tijink, M. Wester, G. Glorieux, K.G.F. Gerritsen, J. Sun, P.C. Swart, Z. Borneman, M. Wessling, R. Vanholder, J.A. Joles, D. Stamatialis, Mixed matrix hollow fiber membranes for removal of protein-bound toxins from human plasma, Biomaterials 34(32) (2013) 7819-7828.

[16] D. Pavlenko, E. van Geffen, M.J. van Steenbergen, G. Glorieux, R. Vanholder, K.G.F. Gerritsen, D. Stamatialis, New low-flux mixed matrix membranes that offer superior removal of protein-bound toxins from human plasma, Scientific Reports 6(1) (2016) 34429.

[17] C. Ronco, W.R. Clark, Haemodialysis membranes, Nature Reviews Nephrology 14(6) (2018) 394-410.

[18] C. Legallais, D. Kim, S.M. Mihaila, M. Mihajlovic, M. Figliuzzi, B. Bonandrini, S. Salerno, F.A. Yousef Yengej, M.B. Rookmaaker, N. Sanchez Romero, P. Sainz-Arnal, U. Pereira, M. Pasqua, K.G.F. Gerritsen, M.C. Verhaar, A. Remuzzi, P.M. Baptista, L. De Bartolo, R. Masereeuw, D. Stamatialis, Bioengineering organs for blood detoxification, advanced healthcare materials 7(21) (2018) 1800430.

[19] O.E.M. ter Beek, D. Pavlenko, D. Stamatialis, Hollow fiber membranes for long-term hemodialysis based on polyethersulfone-SlipSkin ${ }^{\mathrm{TM}}$ polymer blends, Journal of Membrane Science 604 (2020) 118068.

[20] M.P.M. Graham-Brown, D.R. Churchward, A.C. Smith, R.J. Baines, J.O. Burton, A 4-month programme of in-centre nocturnal haemodialysis was associated with improvements in patient outcomes, Clinical Kidney Journal 8(6) (2015) 1-7.

[21] M. Dam, P.J.M. Weijs, F.J. van Ittersum, B.C. van Jaarsveld, Physical performance in patients treated with nocturnal hemodialysis - A systematic review of the evidence, BMC Nephrology 20(1) (2019) 317.

[22] T. Cornelis, S. Eloot, R. Vanholder, G. Glorieux, F.M. van der Sande, J.L. Scheijen, K.M. Leunissen, J.P. Kooman, C.G. Schalkwijk, Protein-bound uraemic toxins, dicarbonyl stress and advanced glycation end products in conventional and extended haemodialysis and haemodiafiltration, Nephrology Dialysis Transplantation 30(8) (2015) 1395-1402.

[23] A. Davenport, V. Gura, C. Ronco, M. Beizai, C. Ezon, E. Rambod, A wearable haemodialysis device for patients with end-stage renal failure: a pilot study, The Lancet 370(9604) (2007) 2005-2010. 
[24] A. Davenport, Portable and wearable dialysis devices for the treatment of patients with end-stage kidney failure: Wishful thinking or just over the horizon?, Pediatric nephrology 30(12) (2015) 2053-2060.

[25] O.E.M. Ter Beek, D. Pavlenko, D. Stamatialis, Advanced blood purification therapies, Biomedical Membranes and (Bio)Artificial Organs, World Scientific 2017, 59-82.

[26] H. Yatzidis, A convenient haemoperfusion microapparatus over charcoal for the treatment of endogenous and exogenous intoxications. Its use as an artificial kidney, Proceedings of the European Dialysis and Transplantation Association 1 (1964) 83-87.

[27] S.V. Mikhalovsky, Emerging technologies in extracorporeal treatment: focus on adsorption, Perfusion 18 (Suppl 1) (2003) 47-54.

[28] P.K. Tyagi, J.F. Winchester, D.A. Feinfeld, Extracorporeal removal of toxins, Kidney international 74(10) (2008) 1231-1233.

[29] N. Cai, Q. Li, J. Zhang, T. Xu, W. Zhao, J. Yang, L. Zhang, Antifouling zwitterionic hydrogel coating improves hemocompatibility of activated carbon hemoadsorbent, Journal of Colloid and Interface Science 503 (2017) 168-177.

[30] C.A. Howell, S.R. Sandeman, Y. Zheng, S.V. Mikhalovsky, V.G. Nikolaev, L.A. Sakhno, E.A. Snezhkova, New dextran coated activated carbons for medical use, Carbon 97 (2015) 134-146.

[31] I. Geremia, R. Bansal, D. Stamatialis, In vitro assessment of mixed matrix hemodialysis membrane for achieving endotoxin-free dialysate combined with high removal of uremic toxins from human plasma, Acta Biomaterialia 90 (2019) 100-111.

[32] D. Kim, D. Stamatialis, High flux mixed matrix membrane with low albumin leakage for blood plasma detoxification, Journal of Membrane Science (2020) 118187.

[33] S.S. Dukhin, Y. Tabani, R. Lai, O.A. Labib, A.L. Zydney, M.E. Labib, Outside-in hemofiltration for prolonged operation without clogging, Journal of Membrane Science 464 (2014) 173-178.

[34] G. Catapano, A. Wodetzki, U. Baurmeister, Blood flow outside regularly spaced hollow fibers: the future concept of membrane devices?, The International Journal of Artificial Organs 15(6) (1992) 327-330.

[35] W.H. Fissell, S. Roy, A. Davenport, Achieving more frequent and longer dialysis for the majority: wearable dialysis and implantable artificial kidney devices, Kidney International 84(2) (2013) 256-264.

[36] E.A. Friedman, Will nephrologists use a wearable artificial kidney?, Clinical Journal of the American Society of Nephrology 4(9) (2009) 1401-1402. 
[37] R.L. Stephens, S.C. Jacobsen, E. Atkin-thor, W. Kolff, Portable/wearable artificial kidney (WAK) - initial evaluation, Proceedings of the European Dialysis and Transplantation Association 12 (1976) 511-518.

[38] V. Gura, M. Beizai, C. Ezon, H.D. Polaschegg, Continuous renal replacement therapy for end-stage renal disease. The wearable artificial kidney (WAK), Contributions to Nephrology 149 (2005) 325-333.

[39] D.B. Lee, M. Roberts, A peritoneal-based automated wearable artificial kidney, Clinical and Experimental Nephrology 12(3) (2008) 171-180.

[40] C. Ronco, L. Fecondini, The Vicenza wearable artificial kidney for peritoneal dialysis (ViWAK PD), Blood purification 25(4) (2007) 383-388.

[41] E.L. Penne, L. Visser, M.A. van den Dorpel, N.C. van der Weerd, A.H. Mazairac, B.C. van Jaarsveld, M.G. Koopman, P. Vos, G.W. Feith, T.K. Kremer Hovinga, H.W. van Hamersvelt, I.M. Wauters, M.L. Bots, M.J. Nubé, P.M. Ter Wee, P.J. Blankestijn, M.P. Grooteman, Microbiological quality and quality control of purified water and ultrapure dialysis fluids for online hemodiafiltration in routine clinical practice, Kidney international 76(6) (2009) 665-672.

[42] J.W.M. Agar, Green dialysis: the environmental challenges ahead, Seminars in Dialysis 28(2) (2015) 186192.

[43] C. Boccato, R. Lucena, J. Vienken, Water and Dialysis Fluids: A Quality Management Guide, Pabst Science Publishers, 2015.

[44] R. Bambauer, J. Walther, S. Meyer, S. Ost, M. Schauer, W.K. Jung, H. Göhl, J. Vienken, Bacteria - and endotoxin-free dialysis fluid for use in chronic hemodialysis, Artificial Organs 18(3) (1994) 188-192.

[45] B. Madsen, D.W. Britt, C.H. Ho, M. Henrie, C. Ford, E. Stroup, B. Maltby, D. Olmstead, M. Andersen, Hemodialysis membrane surface chemistry as a barrier to lipopolysaccharide transfer, Journal of Applied Polymer Science 132(21) (2015).

[46] G. Lonnemann, The quality of dialysate: An integrated approach, Kidney International 58 (2000) S112S119.

[47] A. Sinha, A. Bagga, Maintenance dialysis in developing countries, Pediatric Nephrology 30(2) (2015) 211-219.

[48] P. Susantitaphong, C. Riella, B.L. Jaber, Effect of ultrapure dialysate on markers of inflammation, oxidative stress, nutrition and anemia parameters: a meta-analysis, Nephrology Dialysis Transplantation 28(2) (2013) 438-446.

[49] M.B. Gorbet, M.V. Sefton, Endotoxin: the uninvited guest, Biomaterials 26(34) (2005) 6811-6817. 
[50] E. Schepers, G. Glorieux, S. Eloot, M. Hulko, A. Boschetti-de-Fierro, W. Beck, B. Krause, W. Van Biesen, Assessment of the association between increasing membrane pore size and endotoxin permeability using a novel experimental dialysis simulation set-up, BMC Nephrology 19(1) (2018) 1.

[51] G. Lonnemann, L. Sereni, H.D. Lemke, C. Tetta, Pyrogen retention by highly permeable synthetic membranes during in vitro dialysis, Artificial Organs 25(12) (2001) 951-960.

[52] V. Weber, I. Linsberger, E. Rossmanith, C. Weber, D. Falkenhagen, Pyrogen transfer across high- and low-flux hemodialysis membranes, Artificial Organs 28(2) (2004) 210-217.

[53] M.K. van Gelder, J.A.W. Jong, L. Folkertsma, Y. Guo, C. Blüchel, M.C. Verhaar, M. Odijk, C.F. Van Nostrum, W.E. Hennink, K.G.F. Gerritsen, Urea removal strategies for dialysate regeneration in a wearable artificial kidney, Biomaterials 234 (2020) 119735.

[54] R. Vanholder, T. Gryp, G. Glorieux, Urea and chronic kidney disease: the comeback of the century? (in uraemia research), Nephrology Dialysis Transplantation 33(1) (2018) 4-12.

[55] M. Roberts, The regenerative dialysis (REDY) sorbent system, Nephrology 4(4) (1998) 275-278.

[56] T. Alizadeh, M. Akhoundian, Z. Movahed, Indirectly determination of urea using chloride ion selective electrode after its extraction and preconcentration by molecularly imprinted polymer, 1 (2009) 246-256.

[57] T. Alizadeh, Preparation of molecularly imprinted polymer containing selective cavities for urea molecule and its application for urea extraction, Analytica Chimica Acta 669(1) (2010) 94-101.

[58] B. Khadro, C. Sanglar, A. Bonhomme, A. Errachid, N. Jaffrezic-Renault, Molecularly imprinted polymers (MIP) based electrochemical sensor for detection of urea and creatinine, Procedia Engineering 5 (2010) 371374.

[59] C.H. Ooi, W.K. Cheah, Y.L. Sim, S.Y. Pung, F.Y. Yeoh, Conversion and characterization of activated carbon fiber derived from palm empty fruit bunch waste and its kinetic study on urea adsorption, Journal of Environmental Management 197 (2017) 199-205.

[60] C. Giordano, R. Esposito, P. Bello, E. Quarto, F.M. Gonzalez, Cold carbon apparatus for hemodialysis, Journal of Dialysis 1(2) (1976) 165-179.

[61] J. Kim, J. Kim, J.H. Moon, J. Park, K. Lee, E. Kang, H.C. Kim, B. Min, C. Ronco, Development of a cold dialysate regeneration system for home hemodialysis, Blood Purification 28 (2009) 84-92.

[62] W.K. Cheah, Y.L. Sim, F.Y. Yeoh, Amine-functionalized mesoporous silica for urea adsorption, Materials Chemistry and Physics 175 (2016) 151-157. 
[63] V. Wernert, O. Schäf, H. Ghobarkar, R. Denoyel, Adsorption properties of zeolites for artificial kidney applications, Microporous and Mesoporous Materials 83(1) (2005) 101-113.

[64] F. Meng, M. Seredych, C. Chen, V. Gura, S. Mikhalovsky, S. Sandeman, G. Ingavle, T. Ozulumba, L. Miao, B. Anasori, Y. Gogotsi, MXene sorbents for removal of urea from dialysate: a step toward the wearable artificial kidney, ACS Nano 12(10) (2018) 10518-10528.

[65] J. Liu, X. Chen, Z. Shao, P. Zhou, Preparation and characterization of chitosan/Cu(II) affinity membrane for urea adsorption, Journal of Applied Polymer Science 90(4) (2003) 1108-1112.

[66] Y.G. Zhou, Y.D. Yang, X.M. Guo, G.R. Chen, Effect of molecular weight and degree of deacetylation of chitosan on urea adsorption properties of copper chitosan, Journal of Applied Polymer Science 89(6) (2003) $1520-1523$.

[67] A. Pathak, S.K. Bajpai, Preparation of Cu(II)-Immobilized Chitosan (CIC) and preliminary urea uptake study, Polymer-Plastics Technology and Engineering 47(9) (2008) 925-930.

[68] L.D. Wilson, C. Xue, Macromolecular sorbent materials for urea capture, Journal of Applied Polymer Science 128(1) (2013) 667-675.

[69] R. Smakman, A.W. van Doorn, Urea removal by means of direct binding, Clinical Nephrology 26 (Suppl. 1) (1986) $58-62$.

[70] J.A.W. Jong, Y. Guo, D. Hazenbrink, S. Douka, D. Verdijk, J. van der Zwan, K. Houben, M. Baldus, K.C. Scheiner, R. Dalebout, M.C. Verhaar, R. Smakman, W.E. Hennink, K.G.F. Gerritsen, C.F. van Nostrum, A ninhydrin-type urea sorbent for the development of a wearable artificial kidney, Macromolecular Bioscience 20(3) (2020) 1900396. 



\title{
Chapter 2
}

\section{In vitro assessment of mixed matrix hemodialysis membrane for achieving endotoxin-free dialysate combined with high removal of uremic toxins from human plasma}

\author{
I. Geremia ${ }^{a}$, R. Bansal ${ }^{b}$, D. Stamatialis ${ }^{a}$
}

${ }^{a}$ (Bio)artificial organs and ${ }^{b}$ Targeted therapeutics, Department of Biomaterials Science and Technology, TechMed Centre, Faculty of Science and Technology, University of Twente, P.O. Box 217, 7500 AE Enschede, The Netherlands

This chapter has been published:

I. Geremia, R. Bansal, D. Stamatialis, In vitro assessment of mixed matrix hemodialysis membrane for achieving endotoxin-free dialysate combined with high removal of uremic toxins from human plasma, Acta Biomaterialia, 2019, 90, 100-111 


\section{Abstract}

For a single hemodialysis session nearly 500 liters of water are consumed for obtaining pyrogenfree dialysate. However, many efforts are required to avoid biofilm formation in the system and risk of contamination can persist. Water scarcity and inadequate water purification facilities worsen contamination risk in developing countries. Here, we investigated the application of an activated carbon (AC)/polyethersulfone/polyvinylpyrrolidone mixed matrix membrane (MMM) for achieving for the first time endotoxin-free dialysate and high removal of uremic toxins from human plasma with a single membrane. The MMM, thanks to sorbent AC, can remove approximately 10 times more endotoxins from the dialysate compared to commercial fibers. Pyrogens transport through the MMM was investigated analyzing inflammation in THP-1 monocytes incubated with samples from the dialysis circuit, revealing safety-barrier properties of the MMM. Importantly, endotoxins from dialysate and protein-bound toxins from human plasma can be removed simultaneously without compromising AC adsorption capacity. We estimate that only $0.15 \mathrm{~m}^{2}$ of MMM is needed to totally remove the daily production of the protein-bound toxins indoxyl sulfate and hippuric acid and to completely remove endotoxins in a wearable artificial kidney (WAK) device. Our results could open up new possibilities for dialysis therapy with low water consumption including WAK and where purity and scarcity of water are limiting factors for hemodialysis treatment. 


\section{Introduction}

End-stage kidney disease (ESKD) is characterized by a prominent loss of kidney function and renal replacement therapies (RRT) are the only solution for patients' survival. In fact, ESKD patients waiting for or not suitable for kidney transplantation need to undergo dialysis therapy, which is life-sustaining, but very costly and affects their quality of life significantly. The prevalence of ESKD is expected to rise over the next few decades, driven by population ageing and increased prevalence of diabetes and hypertension [1]. As a consequence of this, more affordable therapies are demanded [1].

During dialysis, a semi-permeable membrane is responsible for the transfer of accumulated uremic solutes from blood compartment to the dialysate compartment. To ensure quality and safety of the dialysate (also called dialysis fluid), dialysis clinics need to fulfill strict hygienic rules and to operate expensive multi-step water treatments [2-4]. However, high costs, high energy consumption and, mostly, a large consumption of water are required $[5,6]$. Indeed, for each patient treatment nearly 500 liters of tap water are needed to obtain pure dialysis water, with two thirds of it discharged to the drain $[5,6]$. Moreover, even when ultra-pure water is obtained, inadequate design of the tubing system and improper machine maintenance might lead to water stagnation resulting in microbiological biofilm formation $[2,7-9]$. The situation could be more dramatic in developing countries where the dialysis units have inadequate facilities for water purification [10]. As a consequence of bacterial growth and lysis in the water purification systems, bacterial pyrogens could be released and transferred into the patients' blood [5, 7, 11-13]. A continued exposure to low levels of endotoxins contributes to the micro-inflammatory status of the hemodialysis patients leading to cardiovascular side effects, such as inflammatory amyloidosis and atherosclerosis, while exposure to higher level of endotoxins results in acute side effects as fever, hypotension, headache and muscular cramps [4, 5, 7-9, 11, 14]. 
To minimize the risks of dialysate contamination, additional filters can be inserted at the end of the purification cascade to avoid bacterial components, still present in the system, to reach the dialysis membrane [15-17]. Additionally, several sorbent systems have been studied for removing endotoxins from dialysate and water solution, such as functionalized nanoparticles, plasma exchange, immobilized polymyxin B, poly-L-lysine, di-ethyl aminoethane, histamine, histidine and activated carbon (AC) [8, 18-21]. Besides, several studies have also investigated the ability of the dialysis filter itself to act as protective barrier for pyrogens during the therapy [14, 22]. All the above studies, however, do not discuss the impact of endotoxins removal on the simultaneous removal of uremic toxins from the patients' blood by the membrane.

In the next years, the application of a continuous dialysis therapy outside the hospital, for example portable (PAK) and/or wearable (WAK) artificial kidney devices, together with an improvement of the quality of life of ESKD patients, would need to include a reduction of water requirements for dialysis, leading to a more affordable and eco-friendly therapy [23-27]. In the WAK systems, a small volume of spent dialysate (dialysate containing uremic toxins transferred from blood) needs to be continuously regenerated and reused [27-29]. The continuous recirculation of the dialysate for prolonged time demands additional efforts and technical developments to avoid microbial contamination, without extra water consumption and without affecting the overall weight of the system, to guarantee portability and wearability. For these systems, the development of strategies for achieving endotoxin-free dialysate and for preventing transfer of endotoxins to the blood side would be very important. Preferably, these strategies should not compromise the uremic toxins removal by the system.

Recently, we have successfully developed mixed matrix membrane (MMM) for achieving high removal of a broad range of uremic toxins from human plasma, including protein-bound toxins (PBTs) which cannot be removed effectively by the current dialysis membranes [30-32]. The 
MMM is a dual layer polymeric hollow fiber (HF) membrane consisting of an inner selective layer composed of polyethersulfone (PES) and polyvinylpyrrolidone (PVP) blend and an outer layer composed of AC microparticles embedded in PES/PVP matrix. The MMM combines the benefits of diffusion and convection, provided by the membrane structure, and adsorption, achieved by AC particles dispersed through the membrane. The inner particle-free layer avoids direct contact of the AC with the patient blood and/or plasma providing excellent membrane blood compatibility, as was proven by performing extensive blood compatibility study following the ISO protocol 10993-4 (see Chapter 3). This study revealed that the MMM has low drop in white blood cells and platelet count, low thrombin-antithrombin III-complex (TAT) and complement activation (C5a generation) and causes no hemolysis. In fact, the MMM blood compatibility is comparable to reference commercial dialysis fibers used currently in the clinic (see chapter 3 ). In this study, we investigate the application of the MMM for achieving complete removal of endotoxins from dialysate and preventing transfer of endotoxins to the blood compartment while at the same time achieving high removal of uremic toxins from human plasma. In fact, we investigate the adsorption in vitro of lipopolysaccharide (LPS) from E. coli and P. aeruginosa on the MMM in both static and dynamic conditions. Dynamic adsorption of LPS (P. aeruginosa) on the MMM is also investigated in vitro in presence of PBTs in human plasma to study whether the adsorption of PBTs on the AC would compromise the simultaneous removal of endotoxins from the dialysate and vice versa. Diffusion experiments using dialysate model solution contaminated with bacterial culture filtrates from $P$. aeruginosa and S. maltophilia are also performed to assess the ability of the MMM to act as a safety-barrier to avoid transfer of pyrogens to the plasma. A single layer PES/PVP HF membrane without AC particles is used as control and the obtained results are compared with literature studies using current dialysis membranes. To the best of our knowledge, 
In vitro assessment of mixed matrix hemodialysis membrane for achieving endotoxin-free dialysate combined with

high removal of uremic toxins from human plasma

this is the first study which investigates the combined removal of endotoxins and PBTs using a single hemodialysis membrane. 


\section{Materials and methods}

\subsection{Membrane fabrication}

We prepared dual layer hollow fiber (HF) mixed matrix membrane (MMM) with activated carbon (AC) particles and single layer polyethersulfone (PES)/polyvinylpyrrolidone (PVP) HF membrane without particles, as control, via dry-wet spinning technique, as described earlier by Pavlenko et al [30]. Briefly, the polymer dope solutions were prepared by dissolving Ultrason E6020 PES (BASF, Ludwigshafen, Germany) and PVP K90 (molecular weight $\approx 360 \mathrm{kDa}$, Sigma-Aldrich Chemie GmbH, Munchen, Germany) in ultrapure N-methylpyrrolidone (NMP) (Acros Organics, Geel, Belgium). Norit A Supra AC (Norit Netherlands BV, Amersfoort, The Netherlands) was used as sorbent material. It has a BET (Brunauer-Emmett-Teller) surface area of $1700 \mathrm{~m}^{2} / \mathrm{g}$ and it consists of micropores of approximately $0.5 \mathrm{~nm}(<0.7 \mathrm{~nm})$ and $0.9 \mathrm{~nm}$ and small mesopores of about $3 \mathrm{~nm}$ [33]. Prior to use, AC was sieved through $45 \mu \mathrm{m}$ sieve and then was added to the dope solution for preparing the outer layer of the MMM. The concentrations of PES, PVP and AC and the spinning parameters used in the study are specified in Table 1 of Supplementary Information. All polymer solutions were mixed on a roller bench for 3 days, then they were transferred in stainless-steel syringes and left to degas for 24 hours. Afterwards, the syringes were connected to high-pressure syringe pumps and to a designed spinneret for preparing the HF [30]. Ultrapure water was used as bore forming solution. The air-gap between the spinneret and the coagulation bath was adjusted to $10 \mathrm{~cm}$. A collecting wheel was used for the collection of the produced HF. The fabricated membranes were washed with demineralized-water and stored for further use. The single layer PES/PVP HF was produced in the same way as the MMM without the outer layer. 


\subsection{Depyrogenation treatment}

All the glassware, tubing and membranes used for endotoxins removal experiments and for bacterial culture filtrate experiments were subjected to a depyrogenation treatment. The glassware was cyclically washed for 15 minutes with $1 \mathrm{M} \mathrm{NaOH}$ (Sigma-Aldrich Chemie GmbH, Munchen, Germany), $1 \mathrm{M} \mathrm{HCl}$ (Sigma-Aldrich Chemie GmbH, Munchen, Germany) and 95\% EtOH (Boom BV, Meppel, The Netherlands) in ultrasonic bath. Rinsing with endotoxin-free water (Charles River Microbial Solutions, Dublin, Ireland) was performed in between each solution treatment and at the end. Afterwards, the cleaned glassware was left in oven at $180{ }^{\circ} \mathrm{C}$ for at least 3.5 hours and stored in closed containers at $-20{ }^{\circ} \mathrm{C}$ till use. The membranes and the tubing were cyclically flushed for 30 minutes with $1 \mathrm{M} \mathrm{NaOH}, 1 \mathrm{M} \mathrm{HCl}$ and 95\% EtOH. Between each cleaning solution agent and at the end the membranes were flushed with endotoxin-free water (for 15 minutes). Prior to endotoxins adsorption experiments, the water of the last cleaning step (for 30 minutes) was analyzed to confirm that no endotoxins were present in the membranes, tubing or module. After the cleaning treatment, the fibers were immediately used to avoid contamination.

\subsection{Membrane characterization}

\subsubsection{Scanning Electron Microscopy (SEM)}

The morphology of the HF membranes was analyzed by SEM (JEOL JSM-IT 100, Tokyo, Japan). For the imaging of the cross-sections, the membranes were dried in air and fractured in liquid nitrogen. Prior to SEM imaging, the samples were gold sputtered using the Cressington 108 auto sputter (Cressington Scientific Instruments, Watford, UK). 


\subsubsection{Water transport experiments}

Membrane modules composed of $3 \mathrm{HF}$ with total surface area of $2.5 \mathrm{~cm}^{2}$ for the MMM and 3.7 $\mathrm{cm}^{2}$ for the PES/PVP HF were used. A 2-compontent epoxy glue (Griffon Combi Snel-Rapide, Bison International, Goes, The Netherlands) was used for the preparation of the modules. Before water transport experiments, the HF modules $(n=3)$ were depyrogenated and pre-compacted with ultra-pure water at a trans-membrane pressure (TMP) of 2 Bar for 30 minutes. Afterwards, the amount of permeated water was measured over time at TMP of 1, 1.5 and 2 Bar. The resulting water permeance was calculated as the slope of the linear fit of the flux $\left(\mathrm{L} /\left(\mathrm{m}^{2} \cdot \mathrm{h}\right)\right)$ versus the TMP (Bar).

\subsection{Lipopolysaccharide (LPS) adsorption experiments}

\subsubsection{LPS static adsorption}

After the depyrogenation treatment of the samples, $3 \mathrm{mg}$ of the dual layer MMM, $1.5 \mathrm{mg}$ of AC and $1.5 \mathrm{mg}$ of the PES/PVP HF were immersed in $3 \mathrm{~mL}$ of dialysate model solution contaminated with LPS from E. coli 0111:B4 (Sigma Aldrich Chemie GmbH, Schnelldorf, Germany) at a concentration of $405 \pm 249 \mathrm{EU} / \mathrm{mL}$. To prepare the dialysate model solution, $2 \mathrm{mM} \mathrm{KCl,} 140 \mathrm{mM}$ $\mathrm{NaCl}, 1.5 \mathrm{mM} \mathrm{CaCl}_{2}, 0.25 \mathrm{mM} \mathrm{MgCl}, 35 \mathrm{mM} \mathrm{NaHCO}_{3}$ (all from Sigma-Aldrich Chemie GmbH, Schnelldorf, Germany) and $5.5 \mathrm{mM}$ glucose (Life Technologies Europe BV, Bleiswijk, The Netherlands) were dissolved in endotoxin-free water. The samples were left on horizontal shaker at $37^{\circ} \mathrm{C}$ for 3 hours. After 3 hours, samples were collected for LPS quantification. Supernatants of the $\mathrm{AC}$ resuspended in contaminated dialysate model solution were collected via centrifugation. Experiments were performed in triplicate. 


\subsubsection{LPS dynamic adsorption}

After the depyrogenation treatment of the membranes modules and of the tubing system, dynamic adsorption experiments of LPS on the dual layer MMM and on PES/PVP HF were performed in diffusion mode (TMP $=0$ ) with the solutions (see details below) recirculated in counter-current configuration, using a dedicated set up (Convergence, Enschede, The Netherlands). Membrane modules composed of 3 fibers with total surface area of $2.6 \mathrm{~cm}^{2}$ for the MMM and $3.3 \mathrm{~cm}^{2}$ for the PES/PVP HF were used. Phosphate Buffer Saline (PBS, $\mathrm{pH} 7.4)$ was recirculated at a flow rate of $1 \mathrm{~mL} / \mathrm{min}$ in the lumen of the fibers (corresponding to the blood plasma compartment). On the outside of the fibers (corresponding to the dialysate compartment), dialysate model solution contaminated with LPS (E. coli 0111:B4; $590 \pm 103 \mathrm{EU} / \mathrm{mL}$ ) was recirculated at a flow rate of 10 $\mathrm{mL} / \mathrm{min}$ ("outside-in configuration"). To estimate the selectivity of the inner membrane layer for LPS removal, we also performed experiments using the "inside-out configuration". In this case, the PBS was recirculated at $10 \mathrm{~mL} / \mathrm{min}$ flow rate in the dialysate compartment, while the LPS contaminated dialysate model solution was recirculated at $1 \mathrm{~mL} / \mathrm{min}$ in the lumen of the fibers. The experiments were performed for 4 hours and then samples from the dialysate model solution contaminated with LPS were collected for LPS quantification. All the parts of the set-up were connected via PTFE (Polytetrafluoroethylene) tubes and LPS adsorption on the tubing was negligible. Experiments were performed in duplicate. All the adsorption results were normalized to the internal surface areas of the fiber modules. 


\subsection{Bacterial culture filtrate permeability and adsorption using MMM}

2.5.1 In vitro dialysis using dialysate model solution contaminated with bacterial culture filtrate For the preparation of the bacterial culture filtrate, P. aeruginosa (ATCC® 27853 ${ }^{\mathrm{TM}}$, LGC Standards GmbH, Wesel Germany) and S. maltophilia (ATCC ${ }^{\circledR}$ 13637 ${ }^{\mathrm{TM}}$, LGC Standards GmbH, Wesel Germany) were cultured at $37^{\circ} \mathrm{C}$ in tryptic soy broth until the log phase of bacteria growth. The bacteria were ultra-sonicated ( 2 minutes, RT) to induce bacterial disintegration. The obtained lysates were then filtered using decreasing pore sizes filters with the final filter with $0.2 \mu \mathrm{m}$ cutoff (Sartorius, Göttingen, Germany). Equal volumes of the bacterial filtrates were then pooled together and diluted using endotoxin-free dialysate model solution to a final concentration of endotoxins of $50 \mathrm{EU} / \mathrm{mL}$. After depyrogenation of the MMM modules and of the tubing system, in vitro dialysis experiments were performed in diffusion mode $(\mathrm{TMP}=0)$ and in counter-current configuration using a dedicated set up (Convergence, Enschede, The Netherlands). In the lumen of the fibers, 50 $\mathrm{mL}$ of endotoxin-free water was recirculated at a flow rate of $1 \mathrm{~mL} / \mathrm{min}$. On the outside of the fibers, $50 \mathrm{~mL}$ of dialysate model solution challenged with the bacterial filtrate was recirculated at a flow rate of $10 \mathrm{~mL} / \mathrm{min}$. Membrane modules composed of 3 fibers with total surface area of 2.7 $\mathrm{cm}^{2}$ were used. The experiments $(\mathrm{n}=3)$ were performed for 4 hours and samples were taken every hour from the two compartments. The samples were freeze-dried and kept at $-80^{\circ} \mathrm{C}$ till further use.

\subsubsection{Cell culture experiments}

Monocytic human THP-1 cells (ATCC, Rockville, MD, USA), which are a leukemia-derived macrophages cell-line, were used for the cell culture experiments. The cells were cultured in suspension in RPMI-1640 medium supplemented with 10\% FBS (fetal bovine serum) (Lonza, Basel, Switzerland), $100 \mathrm{U} / \mathrm{mL}$ penicillin, $0.1 \mathrm{mg} / \mathrm{mL}$ streptomycin (Sigma-Aldrich, St Louis, 
MO) and $2 \mathrm{mM}$ L-glutamine (GE Healthcare, Little Chalfont, UK) in $\mathrm{CO}_{2}$ incubator at $37{ }^{\circ} \mathrm{C} .5 \mathrm{x}$ $10^{5}$ cells/well were seeded in 24-well plates and cultured overnight with $100 \mathrm{ng} / \mathrm{mL}$ of phorbol $12-$ myristate 13-acetate (PMA) (Cayman Chemicals, Ann Arbor, MI, USA). Afterwards, freeze-dried samples from the lumen and dialysate compartments obtained from the dialysis experiments with bacterial culture filtrate were dissolved in culture media and added to the cells. After 24 hours of incubation, the cells were lysed for quantitative PCR.

\subsubsection{RNA extraction, reverse transcription, quantitative real-time PCR}

In order to investigate whether bacterial material could be transferred from dialysate model solution contaminated with bacterial culture filtrates to the lumen "blood compartment" through the MMM, the induction of inflammatory cytokines/markers i.e. interleukin 1 beta (IL-1 $\beta$ ), tumor necrosis factor alpha (TNF- $\alpha$ ), C-C motif chemokine ligand 2 (CCL2) or macrophage chemotactic protein 1 (MCP1), interleukin 6 (IL-6), inducible nitric oxide synthase (iNOS) and high affinity immunoglobulin gamma Fc receptor 1 (FCGR1) was analyzed in human THP1 monocytes incubated with samples from the in vitro dialysis circuit. Total RNA from THP1 cells was isolated using GenElute Total RNA Miniprep Kit (Sigma-Aldrich Chemie GmbH, Schnelldorf, Germany) according to manufacturer's instructions. The RNA concentration was quantitated by a UV spectrophotometer (NanoDrop Technologies, Wilmington, DE). Total RNA (0.5 $\mu \mathrm{g})$ was reversetranscribed using iScript cDNA Synthesis Kit (Bio-Rad, Hercules, CA). Real-time PCR was performed using 2x SensiMix SYBR and Fluorescein Kit (Bioline, QT615-05, Luckenwalde, Germany), 20 ng cDNA and pre-tested gene-specific primer sets. The cycling conditions for the BioRad CFX384 Real-Time PCR detection system were $95{ }^{\circ} \mathrm{C}$ for $10 \mathrm{~min}, 40$ cycles of $95{ }^{\circ} \mathrm{C} / 15$ sec, $72{ }^{\circ} \mathrm{C} / 15 \mathrm{sec}$ and $58{ }^{\circ} \mathrm{C} / 15$ sec. Finally, cycle threshold $(\mathrm{Ct})$ values were normalized to reference gene GAPDH and fold changes in expression were calculated using the 2- $\Delta \Delta \mathrm{Ct}$ method. 
All the primers were purchased from Sigma-Genosys (The Woodlands, TX, USA). The primer sequences are given in the Supplementary Information Table 2.

\subsection{Combined removal of LPS from dialysate model solution and protein-bound toxins (PBTs)}

\section{from human plasma}

The combined removal of LPS from the dialysate model solution and PBTs from human plasma by the MMM was investigated in diffusion mode $(\mathrm{TMP}=0)$ and in counter-current configuration using a dedicated set up (Convergence, Enschede, The Netherlands). After depyrogenation of the membrane modules and of the tubing system, $50 \mathrm{~mL}$ of human plasma (obtained by healthy donors in compliance with local ethical guidelines - Sanquin, Amsterdam, The Netherlands) spiked with indoxyl sulfate (IS) (Sigma-Aldrich Chemie GmbH, Schnelldorf, Germany) (37 $\pm 3 \mathrm{mg} / \mathrm{L}$ ) and hippuric acid (HA) (Sigma-Aldrich Chemie GmbH, Schnelldorf, Germany) (109 \pm 9 mg/L) was recirculated at a flow rate of $1 \mathrm{~mL} / \mathrm{min}$ in the blood compartment. $50 \mathrm{~mL}$ of dialysate model solution challenged with purified LPS (P. aeruginosa 10, Sigma-Aldrich Chemie GmbH, Schnelldorf, Germany) $(10.2 \pm 1.3 \mathrm{EU} / \mathrm{mL})$ was recirculated at a flow rate of $10 \mathrm{~mL} / \mathrm{min}$ in the dialysate compartment. Membrane modules composed of 3 fibers with a total surface area of 2.6 $\mathrm{cm}^{2}$ for the MMM and $3.5 \mathrm{~cm}^{2}$ for the PES/PVP HF were used. As controls, the diffusion experiments were performed also by recirculating dialysate model solution not contaminated with LPS and healthy plasma not spiked with PBTs, using both MMM and single layer PES/PVP. The experiments were performed for 4 hours and samples were taken every hour from the blood plasma and dialysate compartments for quantification of LPS, IS and HA. Experiments were performed in triplicate, except for IS and HA removal using PES/PVP HF where experiments were performed 


\subsection{LPS and PBTs quantification}

For the quantification of LPS, end-point chromogenic Limulus Amebocyte Lysate (LAL) assay (Charles River Microbial Solutions, Dublin, Ireland), with detection limit of $0.015 \mathrm{EU} / \mathrm{mL}$, was performed according to the manufacturer's guidelines. The concentration of IS and HA were analyzed using reverse-phase high-performance liquid chromatography (HPLC, JASCO, Tokyo, Japan). The concentration of IS was measured by fluorescence $\left(\lambda_{\mathrm{ex}}=272 \mathrm{~nm}, \lambda_{\mathrm{em}}=374 \mathrm{~nm}\right)$. The concentration of HA was measured by UV detection at $245 \mathrm{~nm}$. Before IS and HA quantification, plasma and dialysate samples were deproteinized via heat treatment at $95{ }^{\circ} \mathrm{C}$ for 30 minutes and subsequently filtered through $30 \mathrm{KDa}$ filter (AmiconUltracel-30K, Sigma Aldrich Chemie GmbH, Schnelldorf, Germany).

\subsection{Statistical methods}

All the data are presented as mean \pm SD (standard deviation). Statistical analyses were performed using GraphPad Prism version 5.02 (GraphPad Prism Software, La Jolla, CA, USA). Statistical differences for the experiments i.e. water transport and combined removal of LPS from dialysate model solution and PBTs from human plasma were determined using unpaired students' $t$ test. Multiple comparisons between different groups were performed using one-way analysis of variance (ANOVA) with Bonferroni post-hoc test in order to determine statistical differences for LPS static adsorption and LPS dynamic adsorption experiments. Quantitative real-time PCR results were analyzed for statistical differences using one-way analysis of variance (ANOVA) with Dunnett post-hoc test. Differences were considered significant at $\mathrm{p}<0.05$. 


\section{Results and discussion}

\subsection{Membrane characterization}

\subsubsection{Membrane morphology}

The morphology of the dual layer mixed matrix membrane (MMM) and single layer polyethersulfone (PES)/polyvinylpyrrolidone (PVP) hollow fiber (HF) was investigated using scanning electron microscopy (SEM) imaging (Figure 1).

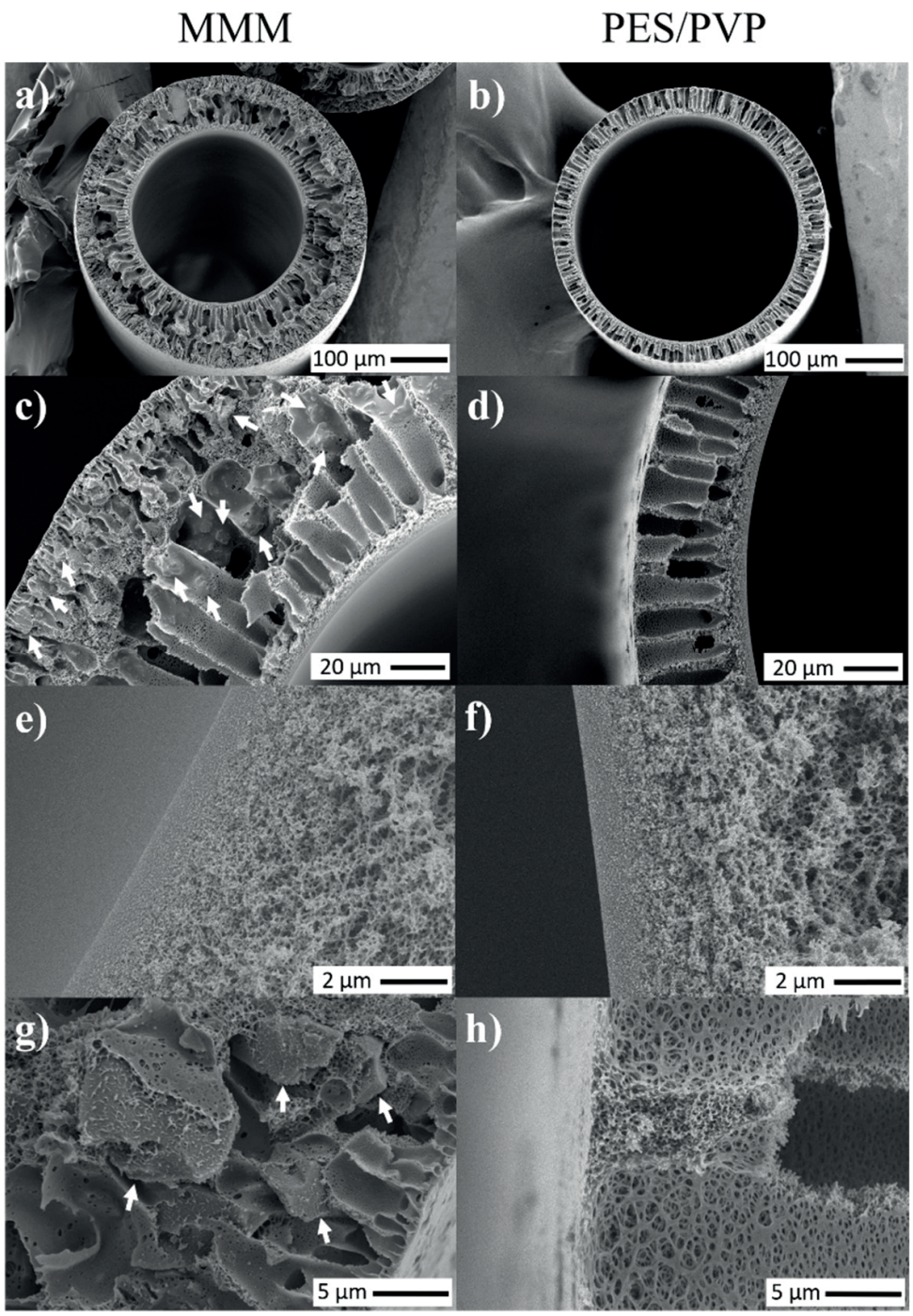

Figure 1. SEM images of the dual layer MMM and PES/PVP HF (a, b: overall cross section; $c$, d: magnification of the wall; e, f: magnification of the inner lumen layer; $g$, h: magnification of the outer layer). White arrows highlight some of the activated carbon (AC) in Fig. 1c and 1g. 
Figure 1 (left) presents the cross-sectional SEM images of the MMM which is a double layer HF composed of an inner particle-free layer and an outer adsorptive layer where activated carbon (AC) particles are dispersed in the polymer matrix. The PES/PVP control membrane is a particle-free single layer HF (Fig. 1, right). The selective layer, responsible for molecule transport via size exclusion, is located on the lumen side (Fig. 1E and 1F) and it looks very similar in morphology and has similar thickness for both membranes, suggesting that similar size-exclusion profiles might be expected for the two membranes. For the MMM, the outer layer (Fig. 1G) (in contact with the dialysate during therapy) contains the AC particles that are dispersed in the polymer matrix (some of the $\mathrm{AC}$ are highlighted in Fig. 1C and $1 \mathrm{G}$ with white arrows). The outer layer of the MMM containing the AC porous particles has larger pore size to allow accessibility and adsorption of the molecules to the sorbent particles and it presents a finger-like macrovoids structure typical of PES membrane. In order to have a low mass transfer resistance and a high adsorption capacity, the MMM has a thinner inner layer compared to the outer MMM layer. Moreover, the two layers are well interconnected, without delamination and visible interface between them. Figures 1B and 1D illustrate that the single layer PES/PVP fiber has also fingerlike macrovoids structure similar to the inner layer of the MMM. The dimensions of the two HF are summarized in Table 1. Overall, due to the presence of the outer adsorptive layer in the MMM, the wall thickness of the MMM is more than double the thickness of the PES/PVP HF wall. However, the transport selectivity is determined by the inner (particle free) layer while the outer layer containing the $\mathrm{AC}$ particles has large pore size and imposes actually very low transport resistance. Moreover, the dimensions of the inner diameter of the two HF are very different, with the PES/PVP HF having an inner diameter 25\% bigger than the MMM. This results in a bigger surface area of PES/PVP hollow fibers when preparing modules for transport and adsorption 
experiments. For this reason, throughout the paper, if not differently indicated, all data have been normalized for the surface area of the membranes in the modules.

Table 1. Dimensions of MMM and PES/PVP HF. The data are expressed as mean $\pm \mathrm{SD}$.

\begin{tabular}{|l|c|c|}
\hline & MMM & PES/PVP \\
\hline Outer diameter $(\mu \mathrm{m})$ & $519 \pm 6$ & $487 \pm 7$ \\
\hline Inner diameter $(\mu \mathrm{m})$ & $306 \pm 2$ & $402 \pm 5$ \\
\hline Inner layer thickness $(\mu \mathrm{m})$ & $46 \pm 7$ & $44 \pm 4$ \\
\hline Outer layer thickness $(\mu \mathrm{m})$ & $64 \pm 4$ & $/$ \\
\hline
\end{tabular}

\subsubsection{Water transport experiments}

The water permeance of the dual layer MMM was found to be equal to $11.4 \mathrm{~L} /\left(\mathrm{m}^{2} \cdot \mathrm{h} \cdot \mathrm{Bar}\right.$ ) (Fig. 2). PES/PVP membrane has higher water permeance $\left(19.5 \mathrm{~L} /\left(\mathrm{m}^{2} \cdot \mathrm{h} \cdot\right.\right.$ Bar $\left.)\right)$ compared to the MMM (Fig. 2). The difference in the water permeance of the two fibers could be due to slightly thinner selective layer and/or surface porosity of the PES/PVP HF. The ultrafiltration coefficients (KUf) of the MMM and PES/PVP membrane are $14.8 \mathrm{~mL} /\left(\mathrm{m}^{2} \cdot \mathrm{h} \cdot \mathrm{mmHg}\right)$ and $25.4 \mathrm{~mL} /\left(\mathrm{m}^{2} \cdot \mathrm{h} \cdot \mathrm{mmHg}\right)$ respectively and they can be both classified as high-flux membranes. Based on the US Food and Drug Administration, $12 \mathrm{~mL} /(\mathrm{h} \cdot \mathrm{mmHg})$ is the value which differentiates low and high-flux dialyzers, whereas in the HEMO study, high-flux dialyzers are defined as having KUf $>14$ $\mathrm{mL} /(\mathrm{h} \cdot \mathrm{mmHg})$; the European Dialysis working group (EUDIAL) defines high-flux dialyzers as having KUf $>20 \mathrm{~mL} /\left(\mathrm{m}^{2} \cdot \mathrm{h} \cdot \mathrm{mmHg}\right)$ [34]. Overall, these results suggest that the MMM has proper morphology characteristics and filtration properties to be used as hemodialysis membrane. 


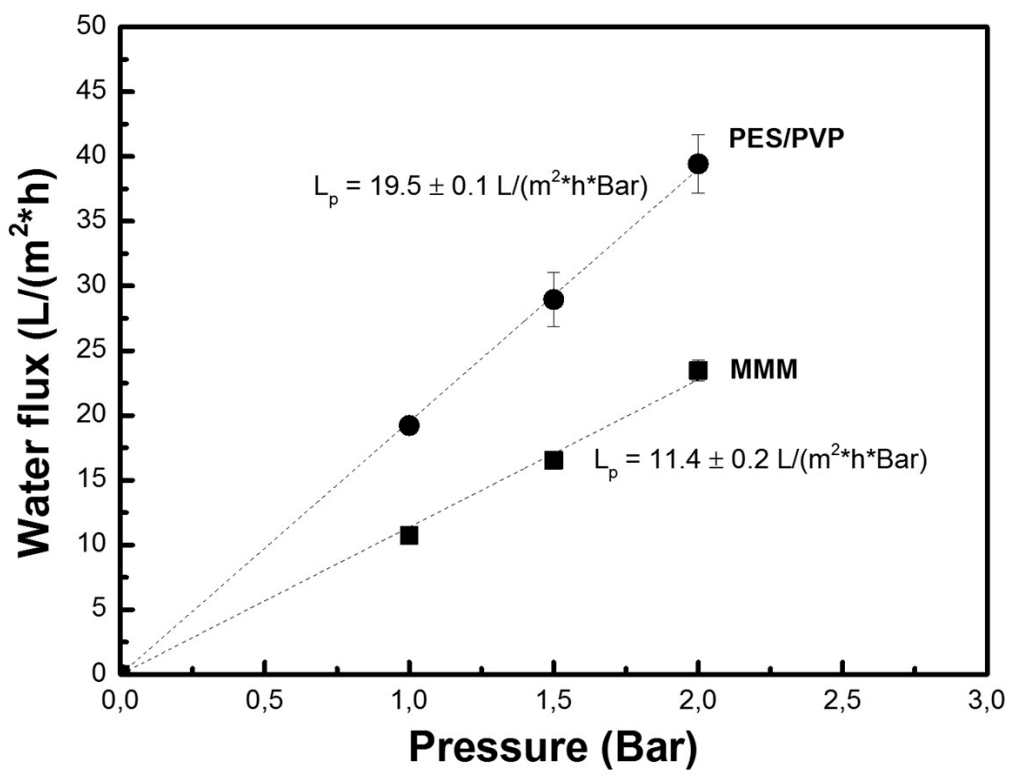

Figure 2. Water permeance results of MMM and PES/PVP HF. The data are expressed as mean $\pm \mathrm{SD}, \mathrm{n}=3$.

\subsection{Lipopolysaccharide (LPS) adsorption experiments}

\subsubsection{Static LPS adsorption}

Figure 3 presents the results of the static adsorption of LPS (E. coli 0111:B4) on MMM, PES/PVP HF and AC particles alone. Table 2 also summarizes the adsorption data for all membranes studied. The adsorption of LPS was found to be significantly higher on the MMM $\left(80 \pm 7 \cdot 10^{3} \mathrm{EU} / \mathrm{g}\right)$ compared to PES/PVP HF $\left(22 \pm 16 \cdot 10^{3} \mathrm{EU} / \mathrm{g}\right)$ due to the presence of AC embedded in the outer layer. By normalizing the amount of LPS adsorbed on the MMM with the mass (in grams) of the $\mathrm{AC}$ embedded in the outer layer of the MMM (52\% of the overall weight of the MMM) and subtracting the average adsorption of LPS on the PES/PVP matrix $\left(22 \cdot 10^{3} \mathrm{EU} / \mathrm{g}\right)$, it is possible to calculate the adsorption of LPS to the AC particles. The AC embedded in the outer layer of the MMM is able to adsorb $131 \pm 13 \cdot 10^{3} \mathrm{EU} / \mathrm{g}$ over a period of 3 hours. No statistical differences were observed between LPS adsorption on the AC embedded in the membrane and the adsorption on 
the AC suspended in solution $\left(158 \pm 0.2 \cdot 10^{3} \mathrm{EU} / \mathrm{g}\right)$ suggesting that the $\mathrm{AC}$ incorporation in the MMM does not compromise its accessibility to LPS.

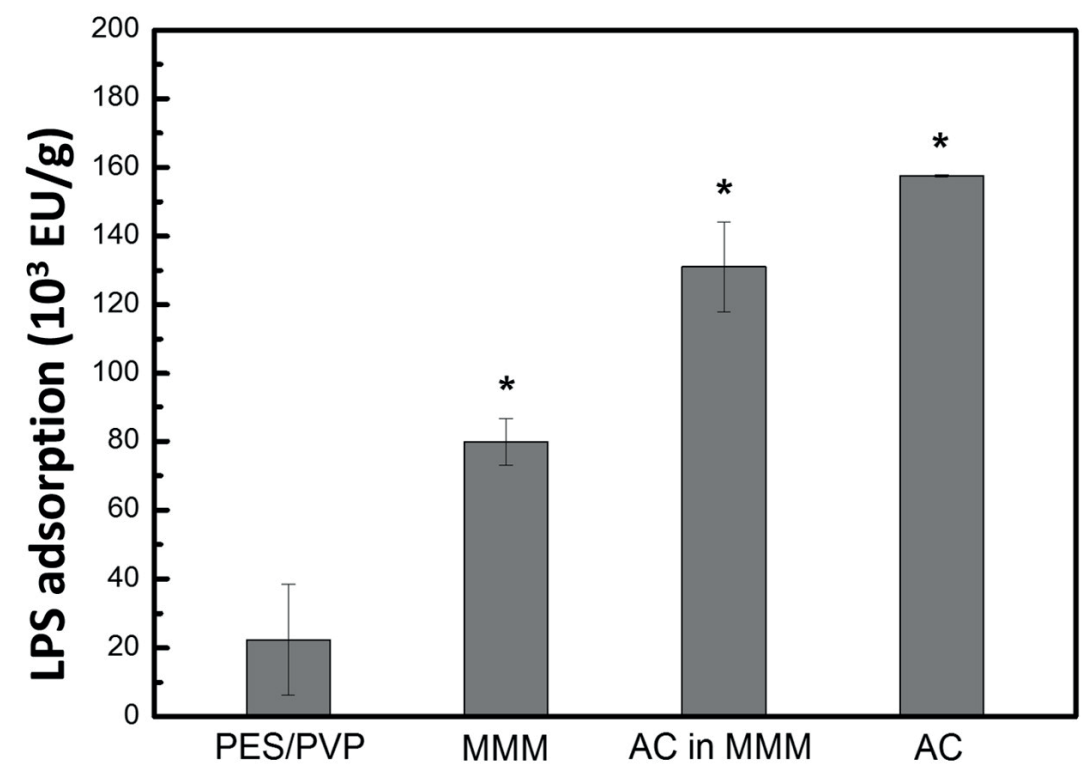

Figure 3. Static adsorption of LPS on PES/PVP HF, on the dual layer MMM, on the AC embedded in the MMM and on $\mathrm{AC}$ alone. Bars represent mean $\pm \mathrm{SD}, \mathrm{n}=3 . * \mathrm{p}<0.05$ denotes significant difference versus PES/PVP. 


\subsubsection{Dynamic LPS adsorption}

a)

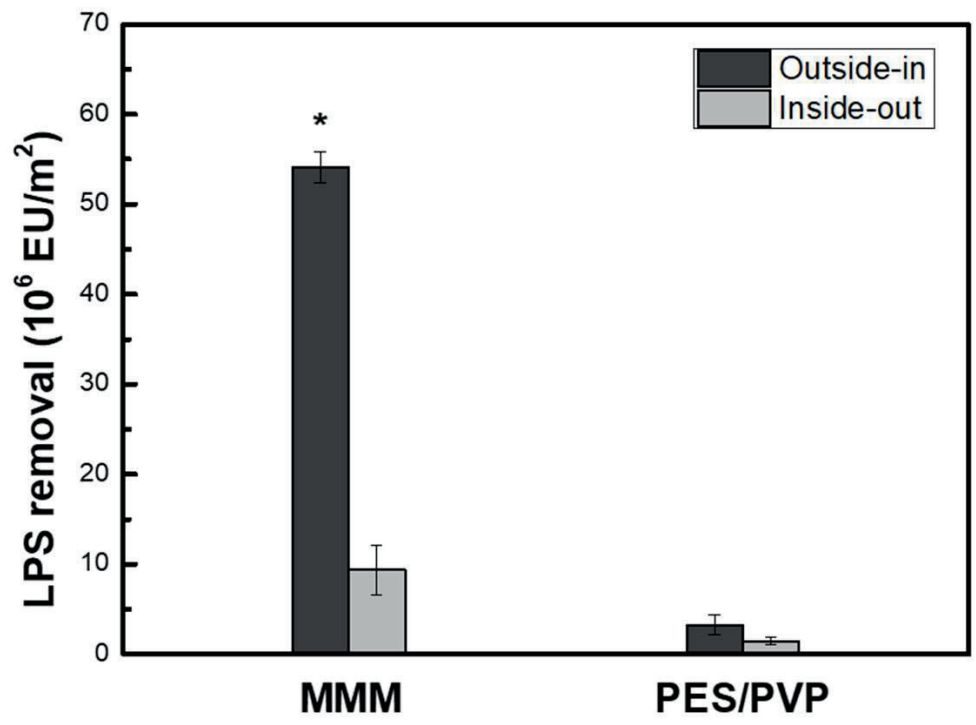

b)
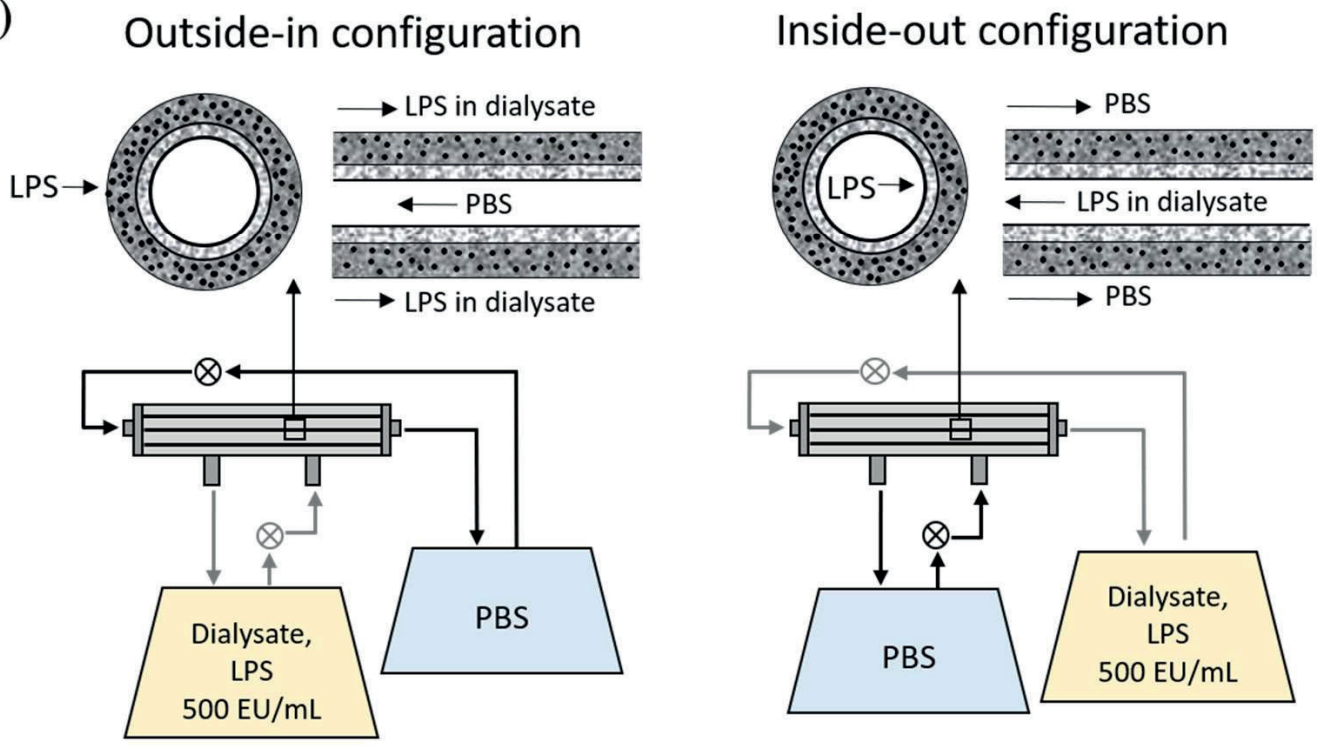

Figure 4. a) Removal of LPS from the dialysate model solution using the dual layer MMM and PES/PVP HF in crossflow diffusion experiment (performed in duplicate) after 4 hours. Bars represent mean $\pm \mathrm{SD},{ }^{*} \mathrm{p}<0.05$ denotes significant difference versus PES/PVP outside-in. b) Experimental set-ups used for LPS dynamic adsorption experiments in outside-in configuration (left) and inside-out configuration (right), see details in Materials and Methods.

Diffusion experiments in outside-in (contaminated dialysate model solution recirculating in the dialysate compartment) and inside-out (contaminated dialysate model solution recirculating in the lumen of the fibers) configurations (Fig. 4b) were performed to study the dynamic adsorption of 
LPS (E. coli 0111:B4) using MMM and PES/PVP HF, and to investigate the contributions of sizeexclusion and adsorption on the overall LPS removal. When the MMM and PES/PVP HF were used in the outside-in configuration, we observed a significantly higher removal of LPS with the $\operatorname{MMM}\left(54.1 \pm 1.8 \cdot 10^{6} \mathrm{EU} / \mathrm{m}^{2}\right)$ compared to the PES/PVP HF $\left(3.6 \pm 1.1 \cdot 10^{6} \mathrm{EU} / \mathrm{m}^{2}\right)($ Fig. $4 \mathrm{a})$ Moreover, the removal of LPS using MMM in outside-in configuration is approximately 6 times higher compared to the MMM in inside-out configuration $\left(9.4 \pm 2.7 \cdot 10^{6} \mathrm{EU} / \mathrm{m}^{2}\right)$ (Fig. $\left.4 \mathrm{a}\right)$. These results indicate that when LPS is recirculated in the dialysate compartment, it can easily access the $\mathrm{AC}$ in the outer layer of the MMM and be highly adsorbed. On the contrary, when LPS is recirculated in the lumen of the MMM (inside-out configuration), LPS is retained by the inner selective layer due to size-exclusion and cannot access the AC dispersed in the outer layer. In fact, the removal of LPS in inside-out configuration using the MMM is comparable to the removal of LPS using the PES/PVP HF in inside-out and outside-in configuration. This indicates that, even though the water permeance of the PES/PVP HF is slightly higher compared to the MMM, the two membranes have similar selectivity, as suggested by the resemblance in the morphology of the selective lumen layers of the two membranes (Fig. 1e and 1f). The LPS removal by the single layer PES/PVP HF in the inside-out $\left(1.5 \pm 0.4 \cdot 10^{6} \mathrm{EU} / \mathrm{m}^{2}\right)$ and outside-in configurations $\left(3.6 \pm 1.1 \cdot 10^{6}\right.$ $\mathrm{EU} / \mathrm{m}^{2}$ ) are also comparable indicating that in the case of the PES/PVP HF LPS is removed via size-exclusion.

For mimicking the clinical situation, LPS removal diffusion experiments in outside-in configuration were also performed using LPS from $P$. aeruginosa 10 (one of the common bacteria found in contaminated dialysate) at a concentration of $10 \mathrm{EU} / \mathrm{mL}$ (similar to the concentrations of endotoxins found in contaminated dialysate in the clinics [9, 35-41]) and recirculating blood 
plasma in the lumen of the fibers. We observed that the MMM removed approximately 390000 EU $/ \mathrm{m}^{2}$ LPS from contaminated dialysate model solution over a period of 2 hours.

It is rather difficult to directly compare our results to other studies because of differences in experimental design, mainly different LPS concentrations and LPS serotype, use of purified LPS or bacteria culture filtrate, as well as operation settings (diffusion and/or convection). In Figure 5, we present a comparative analysis of endotoxins removal at 2 hours by the MMM and commercial fibers $[22,42]$ (all adsorption data were normalized to the membrane surface area). Polyflux $140 \mathrm{H}$, Nephral ST 400, FX 60 and Diacap HI PS18 [42] were studied with an initial concentration of endotoxin (P. aeruginosa) of 50 EU/mL. BLS814G, F60S and Polyflux 14S [22] were tested with endotoxins concentration of $20 \mathrm{EU} / \mathrm{mL}$ (bacterial culture filtrates of $P$. aeruginosa and $S$. maltophilia). LPS concentrations in these studies are higher compared to LPS concentration we used $(10 \mathrm{EU} / \mathrm{mL})$, but still in the same order of magnitude. Nevertheless, it is important to underline that the adsorption capacity is directly related to the concentration. For this reason, we expect higher adsorption of LPS on the MMM if the same LPS concentrations would have been used in our study as for the commercial fibers. Moreover, the commercial fibers used for comparison were all tested for 2 hours, with the first hour in diffusion mode and the second hour in convection mode [22, 42], while the MMM and PES/PVP HF were studied only in diffusion mode. When convection (pressure from the dialysate compartment toward the lumen of the fibers) is used, endotoxins are forced towards the membrane and we would expect higher adsorption of LPS. Independently from the KUf, commercial membranes remove lower than $40000 \mathrm{EU} / \mathrm{m}^{2}$, which is comparable to the removal by our PES/PVP control HF. With this comparative analysis, we conclude that the removal of endotoxins with the MMM is much higher compared to 
commercial fibers. FX60 presents the highest endotoxins removal amongst the commercial hemodialyzers and the MMM can remove about 10 times more endotoxins than FX60 at 2 hours.

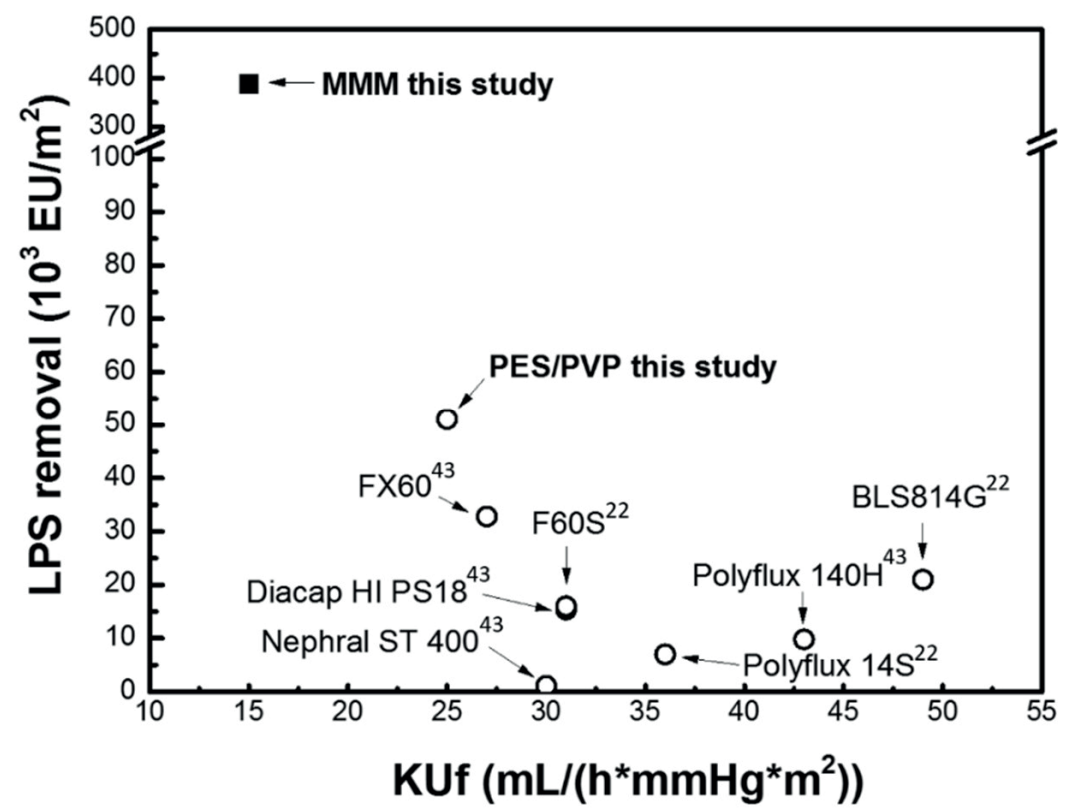

Figure 5. Comparison of endotoxins removal using the MMM and commercial hemodialyzers at 2 hours. References from which the data have been taken are indicated in subscript.

\subsection{Bacterial culture filtrate permeability and adsorption using $M M M$}

Culture bacteria filtrates contain a wide variety of endotoxins fragments with different molecular weights and other bacterial fragments obtained from the disintegration of bacteria cells (such as bacterial DNA, muramylpeptides, peptidoglycans) which are not detected by Limulus Amebocyte Lysate (LAL) test but possess cytokine-inducing activity [9]. P. aeruginosa and S. maltophilia were used for the preparation of the bacterial culture filtrate. These bacteria are amongst the most common bacteria found in water and dialysate in dialysis clinics $[9,22]$. For these tests, endotoxinfree water was recirculated in the "blood compartment" instead of human plasma since in plasma endotoxins and other cytokine-inducing substances may adsorb to different components (such as lipids, hemoglobin, LPS-binding protein and other proteins), thus hampering the detection of their 
trans-membrane flux $[22,36]$. We observed the highly significant upregulation of IL-1 $\beta$, TNF- $\alpha$, CCL2, IL-6, iNOS and FCGR1 mRNA expression when the cells were incubated with the dialysate model solution challenged with bacterial culture filtrate at the start of the experiment (D0h), proving the pronounced ability of the contaminated dialysate model solution to trigger inflammation (Fig. 6). IL-1 $\beta$, TNF- $\alpha$, CCL2, IL-6, iNOS and FCGR1 are the major mediators of inflammation and they are produced by activated monocytes as a consequence of stimulation with pyrogens $[11,12,38]$. IL-1 $\beta$, TNF- $\alpha$, CCL2, IL-6, iNOS and FCGR1 mRNA expression was significantly lower $(\mathrm{p}<0.05)$ in cells incubated with samples from the "blood compartment" (B0h, $\mathrm{B} 1 \mathrm{~h}, \mathrm{~B} 2 \mathrm{~h}, \mathrm{~B} 3 \mathrm{~h}, \mathrm{~B} 4 \mathrm{~h}$ ) and the negative control (medium) compared to the mRNA expression induced by the challenged dialysate model solution at time $0 \mathrm{~h}$ (D0h). Moreover, no significant differences were observed in IL-1 $\beta$, TNF- $\alpha$, CCL2, IL-6, iNOS and FCGR1 mRNA induction when the cells were incubated with samples from the "blood compartment" and the negative control. All these results clearly suggest that no bacterial components could be transferred from dialysate contaminated solution (contaminated with bacterial culture filtrates) to the blood compartment. Therefore, the MMM potentially acts as a safety barrier that is able to avoid the transport of pyrogenic components from the dialysate to the "blood compartment". 

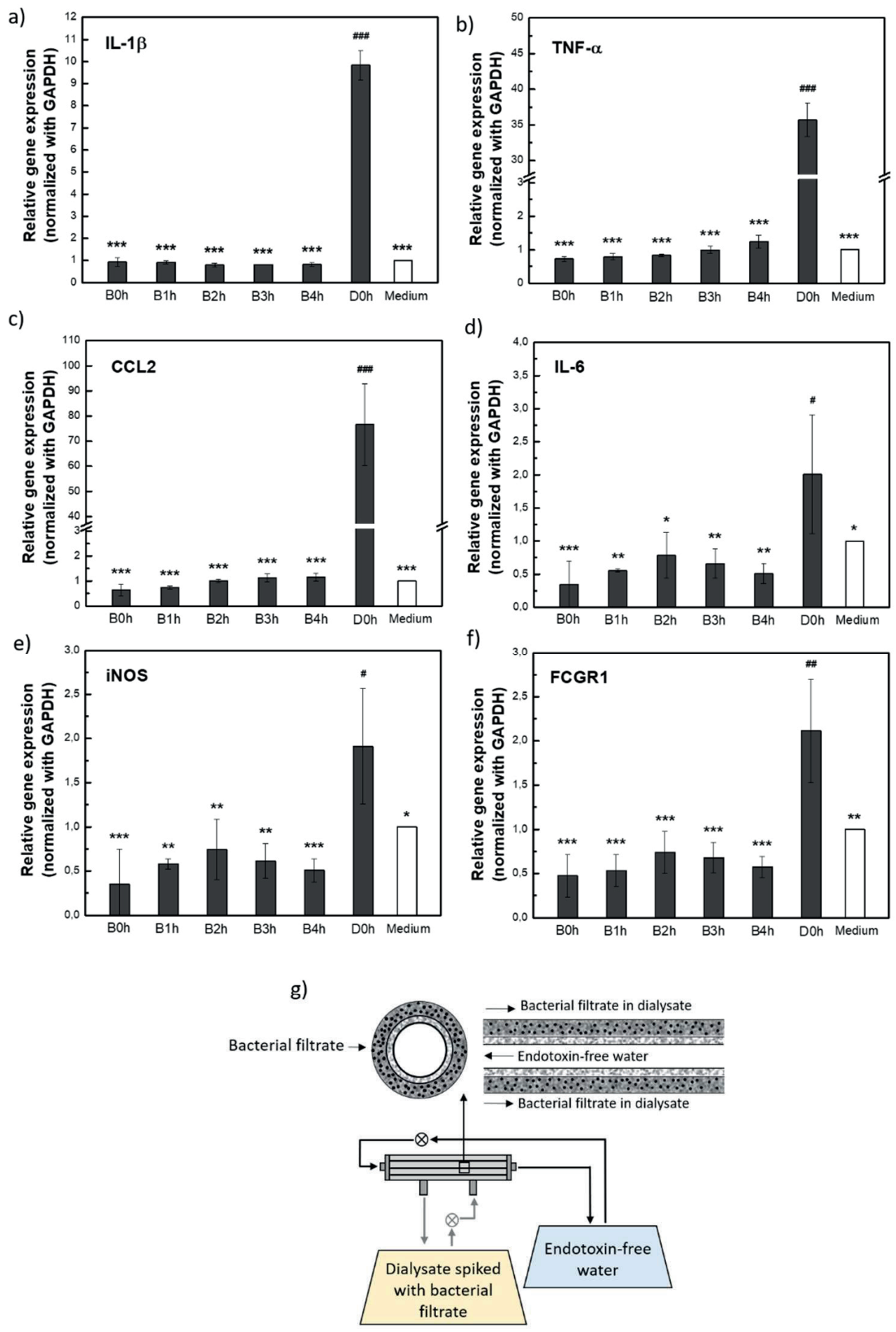

Figure 6. Quantitative mRNA analysis (normalized with GAPDH) of a) IL-1 $\beta$, b) TNF- $\alpha$, c) CCL2, d) IL-6, e) iNOS, f) FCGR1 in THP-1 cells incubated with medium (control) or samples from blood compartment at different time points (B0h, B1h, B2h, B3h, B4h) and with dialysate model solution challenged with the bacterial filtrate at the start of the experiment (D0h). Medium served as the negative control. Bars represent mean $\pm \mathrm{SD}, \mathrm{n}=3 . * \mathrm{p}<0.05, * * \mathrm{p}<$ $0.01, * * * \mathrm{p}<0.001$ denote significant difference versus D0h. \#p $<0.05, \# \# \mathrm{p}<0.01, \# \# \mathrm{p}<0.001$ denote significant difference versus medium control. g) Experimental set-up used to test the permeability and adsorption of bacteria filtrate using the MMM, see details in Materials and Methods. 
Several studies in the literature have investigated the ability of commercial hemodialyzers to avoid back-transport of bacterial disintegration products from the dialysate to the blood. For example, Lonnemann et al. [35] compared the pyrogens permeability of highly permeable DIAPES membrane (DIAPES HF800, Bellco) to polysulfone membrane (BLS 627, Bellco). They observed that a high grade of dialysis fluid contamination caused breakthrough of pyrogens into the blood with both dialyzers, but the highest was found with DIAPES. Henrie et al. [36] tested the endotoxin transfer through various polysulfone and PES synthetic fiber membranes displaying differences in their geometry and KUf. Endotoxin was found in all the blood compartment samples, except for Optiflux F200NR (Fresenius) and for a thick-wall experimental membrane. Madsen et al. [8] investigated the LPS transfer and adsorption of native and modified (varied PVP content, PS-PEG copolymer addition, bleach sterilization) Optiflux F200NR (Fresenius) HF. LPS was not detected in the lumen of the native fibers, but was detected to varying degrees in the lumen of modified fibers. Weber et al. [22] reported significant endotoxin transfer to the blood compartment for both low- and high-flux DIAPES (Bellco) membranes, while Helixone (Fresenius) presented the highest endotoxin adsorption. Schepers et al. [14] compared the permeability of bacterial degradation products using membranes with comparable composition but different pore-size (Poly-flux ${ }^{\circledR} 17 \mathrm{~L}$, Revaclear 400, Theranova 400 and Theralite ${ }^{\mathrm{TM}} 2100$, Baxter). They reported low but detectable amount of endotoxins in the blood compartment with more open membranes [14].

Quantitative mRNA analysis of IL-1 $\beta$, TNF- $\alpha$, CCL2, IL-6, iNOS and FCGR1 was also performed after incubation of THP-1 cells with samples from the dialysate compartment at different time points (Fig. 7). The mRNA induction of IL-1 $\beta$, TNF- $\alpha$ and CCL2 decreases progressively over 
time, being reduced to approximately $60 \%$ after 4 hours for all the three cytokines. In the case of IL-6, iNOS and FCGR1, the mRNA induction is already silenced at 1 h (no statistical difference with control medium). In order to mimic the wearable/portable artificial systems, the contaminated dialysate model solution was continuously recirculated in our in vitro dialysis set-up. In this setting, pyrogens concentration drops on time, since they are adsorbed on the MMM every time the dialysate flows in the module and gets in contact with the MMM. Based on the obtained results (Fig. 7), it is clear that the MMM is able to significantly decrease the pyrogenicity of the dialysate model solution. It also appears that in this experimental setting the MMM is not able to completely eliminate the monocyte activating substances within 4 hours since the expression of IL-1 $\beta$, TNF$\alpha$ and CCL2 mRNA is still significantly higher compared to the negative control condition (medium). Since the removal of the activating substances increases linearly within the 4 hours with no signs of sorbent saturation, we think that by applying longer filtration time and/or by using larger membrane surface area, we could be able to achieve complete elimination of the activating substances. Moreover, the endotoxin concentration in the culture bacterial dialysate model solution was much higher $(50 \mathrm{EU} / \mathrm{mL})$ compared to the endotoxin concentrations usually found in contaminated dialysate in the clinics $(<10 \mathrm{EU} / \mathrm{ml})[9,35,38,40,43]$. 
a)
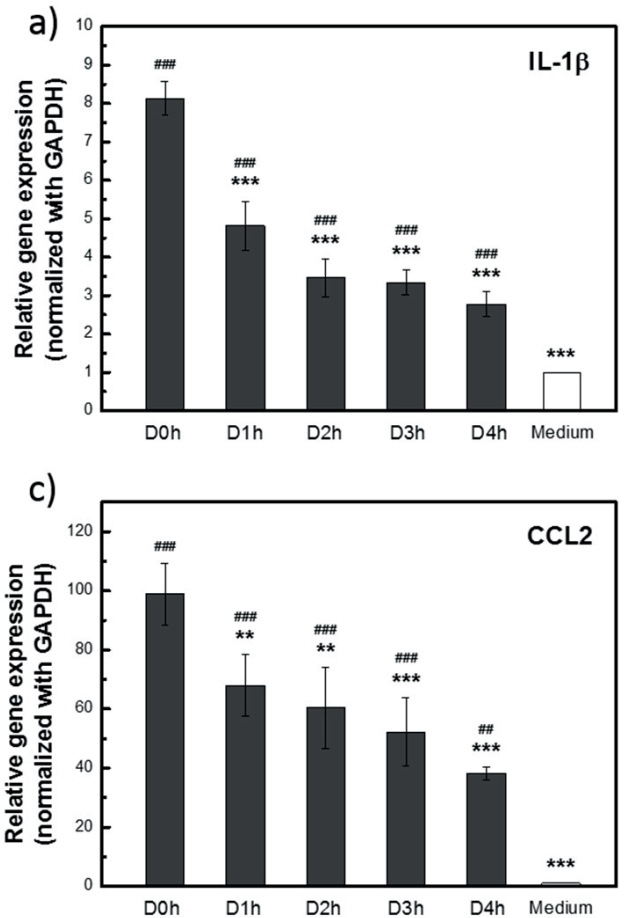

$$
\text { e) }
$$

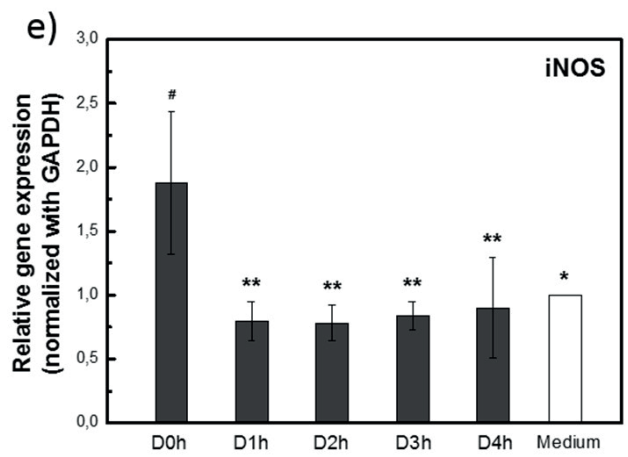

b)

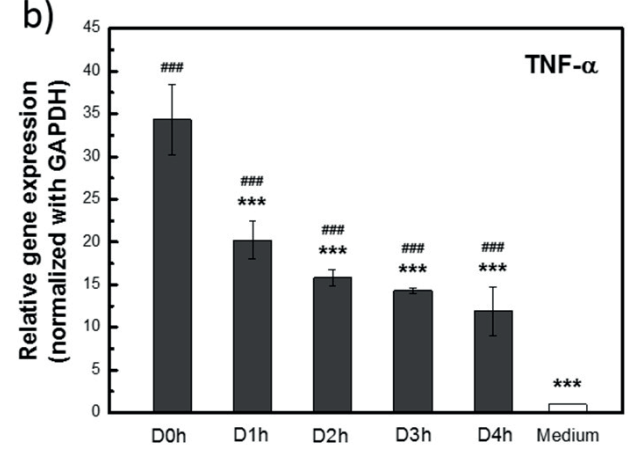

d)

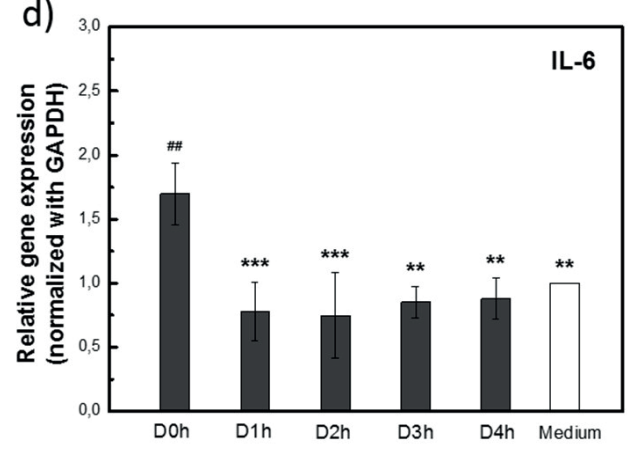

f)

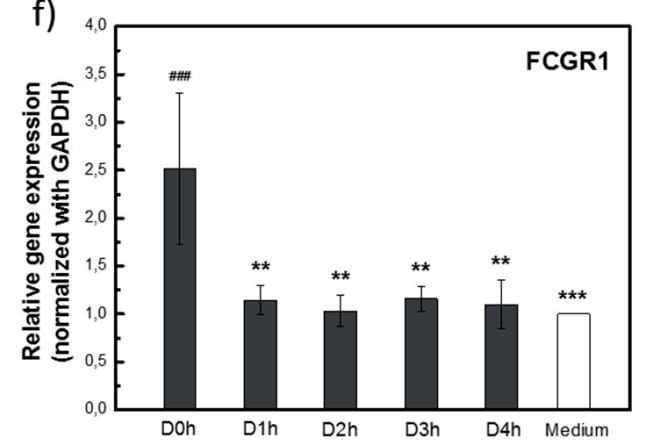

Figure 7. Quantitative mRNA analysis (normalized with GAPDH) of a) IL-1 $\beta$, b) TNF- $\alpha$, c) CCL2, d) IL-6, e) iNOS and f) FCGR1 in THP-1 cells incubated with medium alone (control) or samples from dialysate compartment at different time points (D0h, D1h, D2h, D3h and D4h). Medium served as the negative control. Bars represent mean \pm $\mathrm{SD}, \mathrm{n}=3 .{ }^{*} \mathrm{p}<0.05,{ }^{* *} \mathrm{p}<0.01,{ }^{* * *} \mathrm{p}<0.001$ denote significant difference versus D0h. \#p $<0.05, \# \# \mathrm{p}<0.01, \# \# \mathrm{p}$ $<0.001$ denote significant difference versus medium control. 
a)

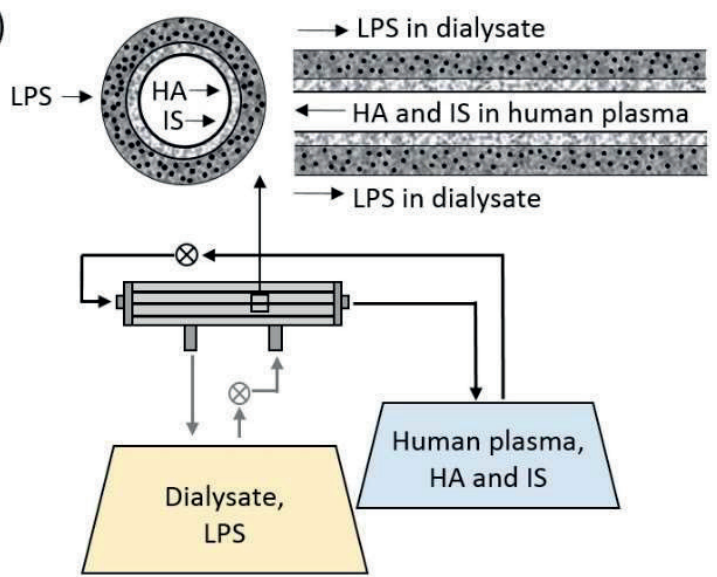

c)

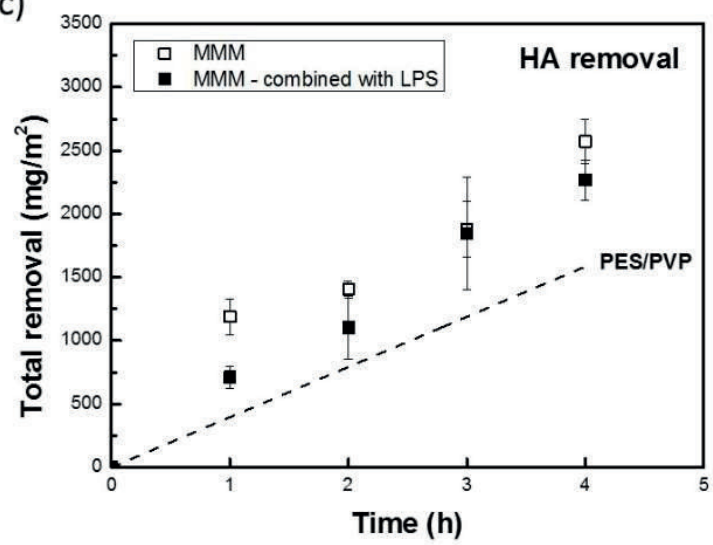

b)

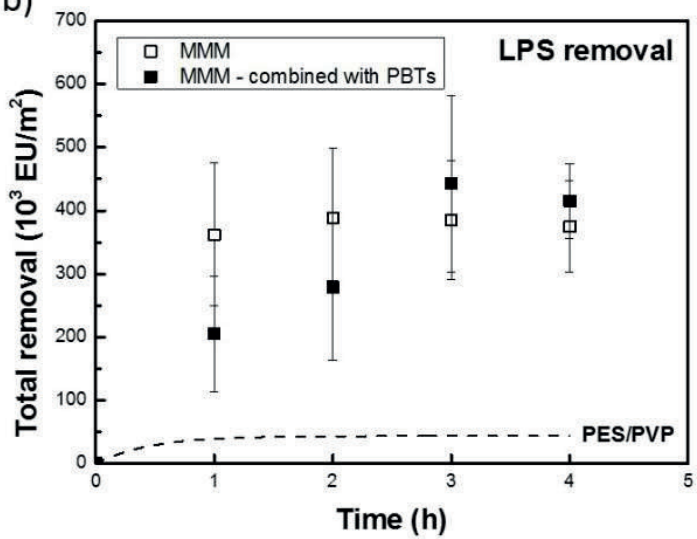

d)

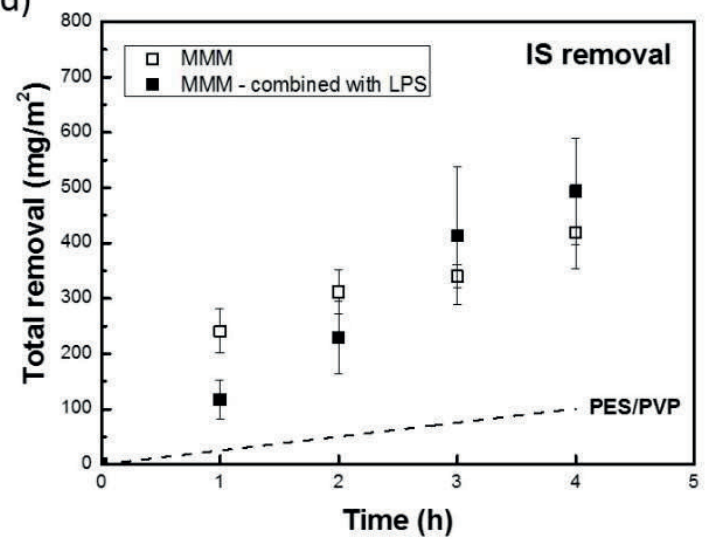

Figure 8. a) Experimental set-up used to test the simultaneous removal of LPS from dialysate model solution and PBTs from human plasma, see details in Materials and Methods. b) LPS removal from dialysate model solution using MMM without HA and IS and combined with HA and IS in the blood compartment and comparison with PES/PVP HF (data fitting); HA (c) and IS (d) total removal using MMM without LPS and combined with LPS in the dialysate model solution and comparison with PES/PVP (data fitting). Data are expressed as mean $\pm \mathrm{SD}, \mathrm{MMM} n=3, \mathrm{PES} / \mathrm{PVP}$ LPS removal $n=3$, PES/PVP HA and IS removal $n=2$.

Due to the high adsorption of LPS on the AC of the MMM, we studied the combined removal of PBTs from human plasma and of LPS ( $P$. aeruginosa 10) from the dialysate to investigate whether the adsorption and retention of LPS from the dialysate can be combined with blood purification. Figure $8 \mathrm{~b}$ presents the LPS removal by the MMM combined or not with the removal of PBTs from human plasma. The LPS removal using the PES/PVP HF is also shown (dotted line, removal at $4 \mathrm{~h}$ 
in Table 2). The MMM is able to remove in both cases approximately $400000 \mathrm{EU} / \mathrm{m}^{2}$ at $4 \mathrm{~h}$ showing that the LPS removal by the MMM is not affected by the simultaneous adsorption of PBTs on the AC. During the first 2 hours, the kinetics of the LPS removal is slower in the presence of indoxyl sulfate (IS) and hippuric acid (HA) in human plasma but with no impact on the total removal in 4h. In the dynamic adsorption experiments (Fig. 4) LPS from E. coli 0111:B4 at a concentration of approximately $500 \mathrm{EU} / \mathrm{mL}$ was used, while the data in Fig. 8b are obtained contaminating the dialysate model solution with LPS from $P$. aeruginosa 10 at a concentration of $10 \mathrm{EU} / \mathrm{mL}$. In both cases, independently of the LPS source, LPS concentration and of the simultaneous removal of PBTs from human plasma, the MMM have 10 times higher removal compared to the PES/PVP HF (Fig. 8b, Table 2).

Figures $8 \mathrm{c}$ and $8 \mathrm{~d}$ show that the removal of IS and HA can be successfully combined to the simultaneous adsorption of LPS on the MMM (no significant difference at 4 h per $\mathrm{p}<0.05$ for HA and IS without and combined with LPS). AC Norit A Supra presents a large BET (BrunauerEmmett-Teller) surface area of $1700 \mathrm{~m}^{2} / \mathrm{g}$ and consists of micropores of approximately $0.5 \mathrm{~nm}$ $(<0.7 \mathrm{~nm})$, micropores of about $0.9 \mathrm{~nm}$ and mesopores of around $3 \mathrm{~nm} \mathrm{[33].} \mathrm{The} \mathrm{molecular} \mathrm{size}$ of LPS can widely differ depending on the bacteria strain and serotype, nevertheless a single LPS molecule (for a $10 \mathrm{KDa}$ unit) has been reported as a cylinder of $9 \mathrm{~nm}$ length and $1 \mathrm{~nm}$ in diameter $[19,44]$. Based on this, we expect that its adsorption on the AC could mainly be attributed to adsorption in the mesopores of AC, while bigger LPS aggregates could adsorb on the external surface of the particles. On the other hand, the smaller micropores would be totally accessible to the smaller IS $(213 \mathrm{~g} / \mathrm{mol})$ and HA $(179 \mathrm{~g} / \mathrm{mol})$ molecules. Importantly, whether or not the dialysate model solution was contaminated with LPS, the removal of the two PBTs does not reach saturation at 4 hours and in both cases the MMM removes more IS and HA compared to the PES/PVP HF (Table 2), which removes the two PBTs only via diffusion. Moreover, due to the fact 
that the two PBTs are bound at different extents to albumin (IS free fraction approximately $9 \%$, HA free-fraction approximately 68\%), adsorption and diffusion contributions on their total removal were found to be different and they were also not altered by the simultaneous removal of LPS by the MMM. In fact, at $4 \mathrm{~h}$ the adsorption contribution on the total removal of IS was about $93 \%$ (7\% diffusion) when pure dialysate was used and $92 \%$ ( $8 \%$ diffusion $)$ when the dialysate was contaminated with LPS. In the case of HA removal, whether or not the dialysate was contaminated with LPS, adsorption and diffusion contributions were equal (approximately $50 \%$ adsorption and $50 \%$ diffusion) on the total removal of HA. These results are consistent with previous studies concerning removal of IS and HA with the MMM [30, 31]. However, our results are achieved in combination to the simultaneous depyrogenation of the dialysate model solution. Assuming similar removal by the MMM in the clinical situation and approximating the daily urinary excretion of IS to $50 \mathrm{mg}[31,45,46]$ and HA to $330 \mathrm{mg}$ [47-49] in omnivorous people, we could estimate that $0.15 \mathrm{~m}^{2}$ surface area of the MMM would be required for the daily removal of IS and HA and to simultaneously totally remove endotoxins from 2 liters (as for WAK systems) of contaminated dialysate (endotoxins concentration $10 \mathrm{EU} / \mathrm{mL}$ ). Comparing to commercial hemodialyzers, which contain 1-2 $\mathrm{m}^{2}$ of membrane, our results show the advantage of applying the MMM in portable and WAK systems. Finally, the endotoxins concentrations found in tap water are reported in the range of 1-30 EU/mL, with 7-8 EU/mL the most common concentrations [50-52], therefore one could anticipate that the MMM could be suitable for artificial kidneys and WAK using tap water. 


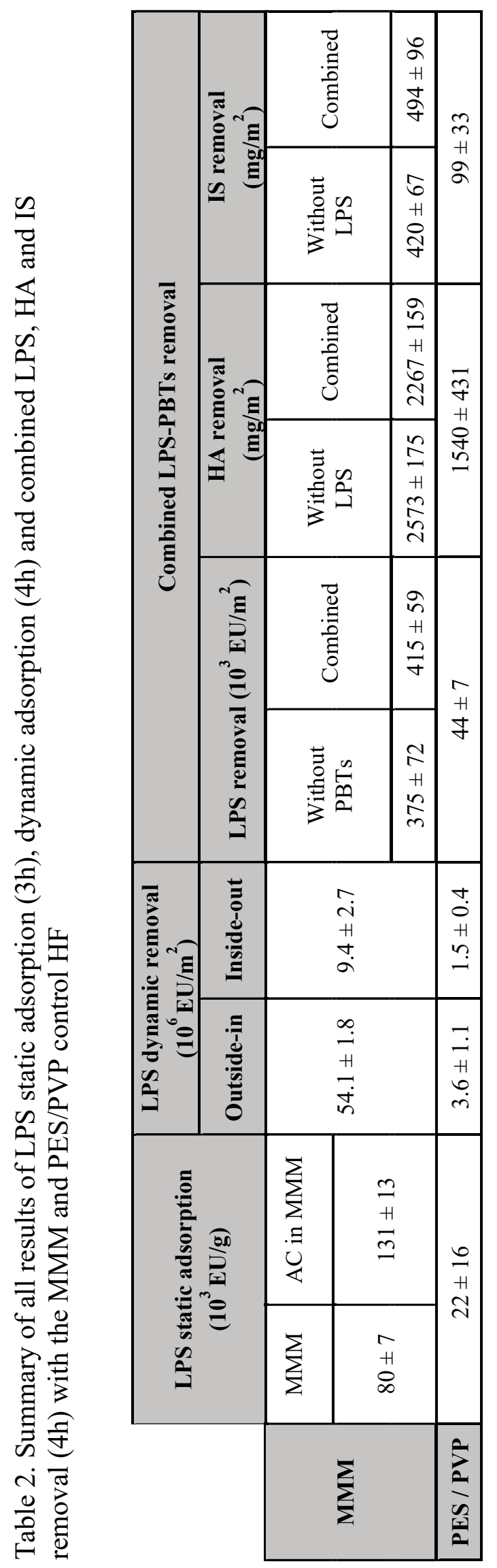




\section{Conclusion and outlook}

In this study, we investigated the application of the MMM for the removal of endotoxins from dialysate combined with high removal of uremic toxins from human plasma.

Our results showed that the MMM offers superior endotoxin removal compared to commercial hemodialyzers and could act as a safe barrier avoiding inflammatory response in case of bacterial contamination of the dialysis system. In fact, based on our results, $0.15 \mathrm{~m}^{2}$ of MMM would be necessary for removing the daily production of IS and HA from human plasma and for simultaneously depyrogenating contaminated dialysate in WAK devices.

Future studies would be focused on the application of the MMM in wearable/portable artificial kidneys and in general for home-hemodialysis treatments, where dialysate should be continuously regenerated and recirculated for long time, thus increasing the risk of bacterial contamination. Moreover, future studies would investigate the application of the MMM for hemodialysis when pure dialysis water (LPS $<0.5 \mathrm{EU} / \mathrm{mL})([53])$ cannot be obtained and used, as for several developing countries affected by water scarcity and having inadequate water purification facilities. Estimation of reduction of waste of water, energy consumption and costs using the MMM would also be assessed.

\section{Acknowledgments}

This work was supported by the European Union's Horizon 2020 research and innovation program under the Marie Skłodowska-Curie grant agreement no. 642890 (http://thelink-project.eu/), and it was partially supported by Health-Holland TKI project NOVAMEM (project no: LSHM16059SGF). 


\section{References}

[1] T. Liyanage, T. Ninomiya, V. Jha, B. Neal, H.M. Patrice, I. Okpechi, M.H. Zhao, J. Lv, A.X. Garg, J. Knight, A. Rodgers, M. Gallagher, S. Kotwal, A. Cass, V. Perkovic, Worldwide access to treatment for end-stage kidney disease: a systematic review, Lancet 385(9981) (2015) 1975-82.

[2] E.L. Penne, L. Visser, M.A. van den Dorpel, N.C. van der Weerd, A.H. Mazairac, B.C. van Jaarsveld, M.G. Koopman, P. Vos, G.W. Feith, T.K. Kremer Hovinga, H.W. van Hamersvelt, I.M. Wauters, M.L. Bots, M.J. Nube, P.M. Ter Wee, P.J. Blankestijn, M.P. Grooteman, Microbiological quality and quality control of purified water and ultrapure dialysis fluids for online hemodiafiltration in routine clinical practice, Kidney International 76(6) (2009) 665-72.

[3] S. Eloot, W. Van Biesen, M. Axelsen, G. Glorieux, R.S. Pedersen, J.G. Heaf, Protein-bound solute removal during extended multipass versus standard hemodialysis, BMC Nephrology 16(1) (2015) 57.

[4] P. Susantitaphong, C. Riella, B.L. Jaber, Effect of ultrapure dialysate on markers of inflammation, oxidative stress, nutrition and anemia parameters: a meta-analysis, Nephrology Dialysis Transplantation 28(2) (2013) 438-446.

[5] C. Boccato, D. Evans, R. Lucena, J. Vienken, Water and Dialysis Fluids, A Quality Management Guide, Pabst Science Publishers, Lengerich, Germany, 2015.

[6] J.W. Agar, Green dialysis: the environmental challenges ahead, Seminars in Dialysis 28(2) (2015) 18692.

[7] R. Bambauer, J. Walther, S. Meyer, S. Ost, M. Schauer, W.K. Jung, H. Gohl, J. Vienken, Bacteria- and endotoxin-free dialysis fluid for use in chronic hemodialysis, Artificial Organs 18(3) (1994) 188-92.

[8] B. Madsen, D.W. Britt, C.-H. Ho, M. Henrie, C. Ford, E. Stroup, B. Maltby, D. Olmstead, M. Andersen, Hemodialysis membrane surface chemistry as a barrier to lipopolysaccharide transfer, Journal of Applied Polymer Science 132(21) (2015) 41550.

[9] G. Lonnemann, The quality of dialysate: an integrated approach, Kidney International Supplements 76 (2000) S112-9.

[10] A. Sinha, A. Bagga, Maintenance dialysis in developing countries, Pediatric Nephrology 30(2) (2015) 211-9. 
[11] M.B. Gorbet, M.V. Sefton, Endotoxin: the uninvited guest, Biomaterials 26(34) (2005) 6811-7.

[12] G. Glorieux, M. Hulko, R. Speidel, K. Brodbeck, B. Krause, R. Vanholder, Looking beyond endotoxin: a comparative study of pyrogen retention by ultrafilters used for the preparation of sterile dialyis fluid, Scientific Reports 4 (2014) 6390.

[13] A. van Tellingen, M.P. Grooteman, M. Schoorl, P.C. Bartels, M. Schoorl, T. van der Ploeg, P.M. ter Wee, M.J. Nube, Intercurrent clinical events are predictive of plasma C-reactive protein levels in hemodialysis patients, Kidney International 62(2) (2002) 632-8.

[14] E. Schepers, G. Glorieux, S. Eloot, M. Hulko, A. Boschetti-de-Fierro, W. Beck, B. Krause, W. Van Biesen, Assessment of the association between increasing membrane pore size and endotoxin permeability using a novel experimental dialysis simulation set-up, BMC Nephrology 19(1) (2018) 1.

[15] C. Weber, W. Groetsch, S. Schlotter, R. Mitteregger, D. Falkenhagen, Novel online infusate-assisted dialysis system performs microbiologically safely, Artificial Organs 24(5) (2000) 323-8.

[16] S. Frinak, H.D. Polaschegg, N.W. Levin, D.J. Pohlod, F. Dumler, L.D. Saravolatz, Filtration of dialysate using an on-line dialysate filter, International Journal of Artificial Organs 14(11) (1991) 691-7.

[17] R. Ouseph, S. Jones, N. Dhananjaya, R.A. Ward, Use of ultrafiltered dialysate is associated with improvements in haemodialysis-associated morbidity in patients treated with reused dialysers, Nephrology Dialysis Transplantation 22(8) (2007) 2269-75.

[18] M.C. Murphy, S. Patel, G.J. Phillips, J.G. Davies, A.W. Lloyd, V.M. Gun'ko, S.V. Mikhalovsky, Adsorption of inflammatory cytokines and endotoxin by mesoporous polymers and activated carbons, in: F. Rodriguez-Reinoso, B. McEnaney, J. Rouquerol, K. Unger (Eds.), Studies in Surface Science and Catalysis, Elsevier 2002, 515-520.

[19] V.M. Gun'ko, W.R. Betz, S. Patel, M.C. Murphy, S.V. Mikhalovsky, Adsorption of lipopolysaccharide on carbon sieves, Carbon 44(7) (2006) 1258-1262.

[20] F.B. Anspach, O. Hilbeck, Removal of endotoxins by affinity sorbents, Journal of Chromatography A 711(1) (1995) 81-92.

[21] R. Darkow, T. Groth, W. Albrecht, K. Lützow, D. Paul, Functionalized nanoparticles for endotoxin binding in aqueous solutions, Biomaterials 20(14) (1999) 1277-1283. 
[22] V. Weber, I. Linsberger, E. Rossmanith, C. Weber, D. Falkenhagen, Pyrogen transfer across high- and low-flux hemodialysis membranes, Artificial Organs 28(2) (2004) 210-7.

[23] A. Davenport, V. Gura, C. Ronco, M. Beizai, C. Ezon, E. Rambod, A wearable haemodialysis device for patients with end-stage renal failure: a pilot study, Lancet 370(9604) (2007) 2005-10.

[24] W.H. Fissell, S. Roy, A. Davenport, Achieving more frequent and longer dialysis for the majority: wearable dialysis and implantable artificial kidney devices, Kidney International 84(2) (2013) 256-64.

[25] V. Gura, C. Ronco, F. Nalesso, A. Brendolan, M. Beizai, C. Ezon, A. Davenport, E. Rambod, A wearable hemofilter for continuous ambulatory ultrafiltration, Kidney International 73(4) (2008) 497-502.

[26] A. Davenport, Portable and wearable dialysis devices for the treatment of patients with end-stage kidney failure: Wishful thinking or just over the horizon?, Pediatric Nephrology 30(12) (2015) 2053-60.

[27] J.P. Kooman, J.A. Joles, K.G. Gerritsen, Creating a wearable artificial kidney: where are we now?, Expert Review of Medical Devices 12(4) (2015) 373-6.

[28] P. Armignacco, A. Lorenzin, M. Neri, F. Nalesso, F. Garzotto, C. Ronco, Wearable devices for blood purification: principles, miniaturization, and technical challenges, Seminars in Dialysis 28(2) (2015) 12530.

[29] P. Armignacco, F. Garzotto, M. Neri, A. Lorenzin, C. Ronco, Wak engineering evolution, Blood Purification 39(1-3) (2015) 110-4.

[30] D. Pavlenko, E. van Geffen, M.J. van Steenbergen, G. Glorieux, R. Vanholder, K.G.F. Gerritsen, D. Stamatialis, New low-flux mixed matrix membranes that offer superior removal of protein-bound toxins from human plasma, Scientific Reports 6 (2016) 34429.

[31] M.S. Tijink, M. Wester, G. Glorieux, K.G. Gerritsen, J. Sun, P.C. Swart, Z. Borneman, M. Wessling, R. Vanholder, J.A. Joles, D. Stamatialis, Mixed matrix hollow fiber membranes for removal of proteinbound toxins from human plasma, Biomaterials 34(32) (2013) 7819-28.

[32] M.S.L. Tijink, M. Wester, J. Sun, A. Saris, L.A.M. Bolhuis-Versteeg, S. Saiful, J.A. Joles, Z. Borneman, M. Wessling, D.F. Stamatialis, A novel approach for blood purification: Mixed-matrix membranes combining diffusion and adsorption in one step, Acta Biomaterialia 8(6) (2012) 2279-2287. 
[33] D. Pavlenko, D. Giasafaki, G. Charalambopoulou, E. van Geffen, K.G.F. Gerritsen, T. Steriotis, D. Stamatialis, Carbon Adsorbents With Dual Porosity for Efficient Removal of Uremic Toxins and Cytokines from Human Plasma, Scientific Reports 7(1) (2017) 14914.

[34] C. Ronco, W.R. Clark, Haemodialysis membranes, Nature Reviews Nephrology 14(6) (2018) 394410.

[35] G. Lonnemann, L. Sereni, H.D. Lemke, C. Tetta, Pyrogen retention by highly permeable synthetic membranes during in vitro dialysis, Artificial Organs 25(12) (2001) 951-60.

[36] M. Henrie, C. Ford, M. Andersen, E. Stroup, J. Diaz-Buxo, B. Madsen, D. Britt, C.H. Ho, In vitro assessment of dialysis membrane as an endotoxin transfer barrier: geometry, morphology, and permeability, Artificial Organs 32(9) (2008) 701-10.

[37] S. Szathmary, E. Hegyi, M.C. Amoureux, N. Rajapakse, L. Chicorka, G. Szalai, K. Reszegi, Z. Derbyshire, J. Paluh, B. Dodson, P. Grandics, Characterization of the DialGuard ${ }^{\mathrm{TM}}$ Device for Endotoxin Removal in Hemodialysis, Blood Purification 22(5) (2004) 409-415.

[38] G. Glorieux, N. Neirynck, N. Veys, R. Vanholder, Dialysis water and fluid purity: more than endotoxin, Nephrology Dialysis Transplantation 27(11) (2012) 4010-21.

[39] R. Vanholder, E. Van Haecke, N. Veys, S. Ringoir, Endotoxin transfer through dialysis membranes: small- versus large-pore membranes, Nephrology Dialysis Transplantation 7(4) (1992) 333-9.

[40] H. Bender, A. Pflazel, N. Saunders, P. Czermak, G. Catapano, J. Vienken, Membranes for endotoxin removal from dialysate: considerations on feasibility of commercial ceramic membranes, Artificial Organs 24(10) (2000) 826-9.

[41] P. Czermak, M. Ebrahimi, G. Catapano, New generation ceramic membranes have the potential of removing endotoxins from dialysis water and dialysate, The International Journal of Artificial Organs 28(7) (2005) 694-700.

[42] H.C. Weber V., Bowry S.K., Endotoxin Permeability oh High-Flux Hemodialysis Membranes Fabricated from Different Polymers, 21st Annual Meeting of the International Society for Blood Purification (ISBP), Blood Purification, 2003, 365-366.

[43] R. Bambauer, M. Schauer, W.K. Jung, V. Daum, J. Vienken, Contamination of dialysis water and dialysate. A survey of 30 centers, ASAIO journal 40(4) (1994) 1012-6. 
[44] M. Kastowsky, T. Gutberlet, H. Bradaczek, Molecular modelling of the three-dimensional structure and conformational flexibility of bacterial lipopolysaccharide, Journal of Bacteriology 174(14) (1992) 4798-806.

[45] E. Cassani, M. Barichella, R. Cancello, F. Cavanna, L. Iorio, E. Cereda, C. Bolliri, P. Zampella Maria, F. Bianchi, B. Cestaro, G. Pezzoli, Increased urinary indoxyl sulfate (indican): New insights into gut dysbiosis in Parkinson's disease, Parkinsonism \& Related Disorders 21(4) (2015) 389-393.

[46] K.P. Patel, F.J. Luo, N.S. Plummer, T.H. Hostetter, T.W. Meyer, The production of p-cresol sulfate and indoxyl sulfate in vegetarians versus omnivores, Clinical Journal of the American Society of Nephrology 7(6) (2012) 982-8.

[47] H.F.N. Kvitvang, T. Andreassen, T. Adam, S.G. Villas-Bôas, P. Bruheim, Highly Sensitive GC/MS/MS Method for Quantitation of Amino and Nonamino Organic Acids, Analytical Chemistry 83(7) (2011) 2705-2711.

[48] S. Bouatra, F. Aziat, R. Mandal, A.C. Guo, M.R. Wilson, C. Knox, T.C. Bjorndahl, R. Krishnamurthy, F. Saleem, P. Liu, Z.T. Dame, J. Poelzer, J. Huynh, F.S. Yallou, N. Psychogios, E. Dong, R. Bogumil, C. Roehring, D.S. Wishart, The Human Urine Metabolome, PLOS ONE 8(9) (2013) e73076.

[49] D. Krupp, N. Doberstein, L. Shi, T. Remer, Hippuric Acid in 24-Hour Urine Collections Is a Potential Biomarker for Fruit and Vegetable Consumption in Healthy Children and Adolescents, The Journal of Nutrition 142(7) (2012) 1314-1320.

[50] D. Simazaki, M. Hirose, H. Hashimoto, S. Yamanaka, M. Takamura, J. Watanabe, M. Akiba, Occurrence and fate of endotoxin activity at drinking water purification plants and healthcare facilities in Japan, Water Research 145 (2018) 1-11.

[51] W.B. Anderson, C.I. Mayfield, D.G. Dixon, P.M. Huck, Endotoxin inactivation by selected drinking water treatment oxidants, Water Research 37(19) (2003) 4553-60.

[52] J. Rapala, K. Lahti, L.A. Rasanen, A.L. Esala, S.I. Niemela, K. Sivonen, Endotoxins associated with cyanobacteria and their removal during drinking water treatment, Water Research 36(10) (2002) 2627-35.

[53] I.O.f. Standardization, ISO 23500: Guidance for the preparation and quality management of fluids for haemodialysis and related therapies, 2014. 


\section{Supplementary Information}

Table SI 1. Spinning conditions of the dual layer MMM

\begin{tabular}{|l|c|}
\hline & Dual layer MMM \\
\hline Inner layer composition & $15 / 7 \mathrm{wt} \%$ \\
\hline Outer layer composition & $14 / 1.4 / 60 \mathrm{wt} \%$ \\
\hline Inner layer pumping speed & $0.4 \mathrm{~mL} / \mathrm{min}$ \\
\hline Outer layer pumping speed & $1.6 \mathrm{~mL} / \mathrm{min}$ \\
\hline Bore liquid & Ultra-pure water \\
\hline Bore liquid pumping speed & $1.2 \mathrm{~mL} / \mathrm{min}$ \\
\hline Air gap & $10 \mathrm{~cm}$ \\
\hline Collecting wheel speed & $8.3 \mathrm{~m} / \mathrm{min}$ \\
\hline
\end{tabular}

Table SI 2. Primer sequences of selected human genes

\begin{tabular}{|c|c|c|}
\hline Gene & Forward $\left(\mathbf{5}^{\prime} \rightarrow \mathbf{3}\right.$ ') & Reverse $\left(\mathbf{3}^{\prime} \boldsymbol{\rightarrow} \mathbf{5}\right.$ ') \\
\hline GAPDH & TCCAAAATCAAGTGGGGCGA & TGATGACCCTTTTGGCTCCC \\
\hline IL-1 $\boldsymbol{\beta}$ & CAGAAGTACCTGAGCTCGCC & AGATTCGTAGCTGGATGCCG \\
\hline TNF- $\boldsymbol{\alpha}$ & CTTCTGCCTGCTGCACTTTG & GTCACTCGGGGTTCGAGAAG \\
\hline CCL2 & GATCTCAGTGCAGAGGCTCG & TTTGCTTGTCCAGGTGGTCC \\
\hline IL-6 & TGCAATAACCACCCCTGACC & ATTTGCCGAAGAGCCCTCAG \\
\hline iNOS & CGCAGAGAACTCAGCCTCAT & CAGGGCTTCAAGAGTTCCGT \\
\hline FCGR1 & AGTCAAGCCTAGCCTGATAATCC & ACATATGCACCCACTCACCTT \\
\hline
\end{tabular}





\title{
Chapter 3
}

\section{Ex vivo evaluation of the blood compatibility of mixed matrix hemodialysis membranes}

I. Geremia ${ }^{\mathrm{a}}$, D. Pavlenko ${ }^{\mathrm{a}}$, K. Maksymow ${ }^{\mathrm{b}}$, M. Rüth ${ }^{\mathrm{b}}$, H.D. Lemke ${ }^{\mathrm{b}}$, D. Stamatialis ${ }^{\mathrm{a}}$

\begin{abstract}
a(Bio)artificial organs, Department of Biomaterials Science and Technology, TechMed Centre, Faculty of Science and Technology, University of Twente, P.O. Box 217, 7500 AE Enschede, The Netherlands

beXcorLab GmbH, Industrie Center Obernburg, Obernburg, Germany.
\end{abstract}

This chapter has been published:

I. Geremia, D. Pavlenko, K. Maksymow, M. Rüth, H.D. Lemke, D. Stamatialis, Ex vivo evaluation of the blood compatibility of mixed matrix hemodialysis membranes, Acta Biomaterialia, 2020, 111, 118-128 


\begin{abstract}
The patients with end stage kidney disease need hemodialysis therapies, using an artificial kidney. Nevertheless, the current therapies cannot remove a broad range of uremic toxins compared to the natural kidney. Adsorption therapies, using sorbent-based columns, can improve the clearance of uremic toxins, but the sorbent particles often require polymeric coatings to improve their hemocompatibility leading to mass transfer limitations and to lowering of their performance.

Earlier, we have developed a dual layer mixed matrix fiber membrane (MMM) based on polyethersulfone/polyvinylpyrrolidone (PES/PVP) polymer blends. There, the sorbent activated carbon particles are embedded in the outer membrane layer for achieving higher removal whereas the inner blood contacting selective membrane layer should achieve optimal blood compatibility. In this work, we evaluate in detail the hemocompatibility of the MMM following the norm ISO 10993-4. We study two generations of MMM having different dimensions and transport characteristics; one with low flux and no albumin leakage and another with high flux but some albumin leakage. The results are compared to those of home-made PES/PVP single layer hollow fiber and to various control fibers already applied in the clinic. Our results show that the low flux MMM successfully avoids contact of blood with the activated carbon and has good hemocompatibility, comparable to membranes currently used in the clinic.
\end{abstract}




\section{Introduction}

A prominent loss of kidney function occurs in end-stage kidney disease (ESKD) patients. Currently, the only solution for patients' survival are renal replacement therapies (RRT) and patients waiting for or not being suitable for kidney transplantation need to undergo dialysis therapy. During hemodialysis, a semi-permeable hollow fiber (HF) membrane is used for the transfer of uremic solutes from the blood compartment to the dialysate compartment of the dialyser. However, the separation profile of hemodialysis membranes has to be improved in order to guarantee the removal of mediumsized and high molecular weight uremic toxins and protein-bound uremic toxins (PBTs) without the leakage of blood proteins through the membrane $[1,2]$.

As an alternative to hemodialysis (HD), hemoperfusion (HP) is an efficient method to remove high quantities of various uremic toxins from blood [3]. Hemoperfusion has been proposed not only for the treatment of ESKD but also for the removal of toxins in poisonings and for the removal of cytokines in septic patients [4-8]. There, the blood can be purified by passing through a column filled with porous sorbent particles [9-11]. However, HP is not designed to correct the fluid balance and the use of small size adsorbent particles may result in high pressure drop across the column which could consequently lead to protein denaturation and/or blood cells damage. Moreover, unless additional filtration is applied, small fragments of the sorbent particles can be leached out of the column and end up in patient blood stream [12,13].

Activated carbon (AC) has a long lasting story of applications in blood purification via HP since it was firstly introduced in 1963 [9, 13]. Nevertheless, its poor hemocompatibility is well known and hemocompatible coatings have to be applied to reduce the side effects of direct AC-blood contact. For example, Cai and co-workers developed a novel antifouling zwitterionic adsorbent to improve hemocompatibility of AC [14]. Sternkopf et al studied the hemocompatibility of activated charcoal coated with polyvinylpyrrolidone for use in extracorporeal blood purification device [15]. Howell and collaborators investigated the hemocompatibility of dextran coated mesoporous AC [16]. These 
methods can improve the hemocompatibility profile of the AC, but often introduce mass transfer limitations which hamper AC adsorption capacity [16].

Recently, we developed the concept of double layer mixed matrix membrane (MMM) based on polyethersulfone/polyvinylpyrrolidone (PES/PVP) polymer blends [17-19]. This is characterized by an inner porous polymeric particle free layer and an outer layer composed of sorbent AC embedded in the polymer matrix. The inner particle-free layer is responsible for the membrane selectivity and prevents contact of the blood with the AC. AC is selected as adsorptive particle because it adsorbs a wide range of solutes, including PBTs, and it has been already widely studied in blood purification $[9,13,20,21]$. Thanks to the presence of the AC in the outer layer of the MMM, diffusion and adsorption can be combined in one step, thus improving the overall toxins removal [17-19]. In fact, due to the presence of $\mathrm{AC}$ in the adsorptive layer, the concentration gradient of the uremic toxins across the membrane is sharp leading to almost 2 times higher toxins removal, ex vivo, in comparison to industrial benchmark membranes [19]. Especially, the MMM is successful in removing PBTs which, although associated with cardiovascular disease, progression of ESKD and mortality [22-29], cannot be removed effectively by the current dialysis membranes [1, 30]. Moreover, in vitro removal of PBTs from blood plasma by the MMM can be combined with high removal of endotoxins from dialysate. Therefore, the MMM could also act as a safe barrier for reducing an inflammatory response within the patients at least in case of bacterial contamination of the dialysate system [31].

The objective of this work is a detailed study of the blood compatibility of the MMM in order to facilitate its clinical implementation. We perform ex vivo experiments, using freshly donated human blood, following the norm ISO 10993-4, as well as characterization of the membrane surface by means of x-ray photoelectron spectroscopy (XPS) and attenuated total reflectance-fourier transform infrared spectroscopy (ATR - FTIR). We investigate two types of MMM, one low-flux and one highflux membrane (indicated as LF-MMM and HF-MMM, respectively) and the results are compared to those of a single layer HF based on PES/PVP (home-made) and to two dialysers, Polysulfone ${ }^{\circledR}$ F60 and Cuprophan ${ }^{\circledR}$ F1. The Polysulfone ${ }^{\circledR}$ F60 membrane is used as negative control due to its excellent 
Chapter 3

track record in clinic, while Cuprophan ${ }^{\circledR} \mathrm{F} 1$ membrane is used as positive control because of its poor hemocompatibility as a consequence of complement activation [32-36]. 


\section{Materials and methods}

\subsection{Membrane fabrication}

We prepared dual layer high-flux mixed matrix membrane (HF-MMM) [17] and low-flux MMM (LF-MMM) [19] hollow fibers (HF) with AC particles and single layer PES/PVP HF control membrane (without particles) via dry-wet spinning technique, as described earlier by Tijink et al and Pavlenko et al [17, 19]. Briefly, the polymer dope solutions were prepared by dissolving polyethersulfone Ultrason E6020 (PES) (BASF, Ludwigshafen, Germany) and polyvinylpyrrolidone (PVP) K90 (molecular weight $\approx 360 \mathrm{kDa}$, Sigma-Aldrich Chemie GmbH, München, Germany) in ultrapure N-methylpyrrolidone (NMP) (Acros Organics, Geel, Belgium). Norit A Supra AC (Norit Netherlands BV, Amersfoort, The Netherlands) was used as sorbent material. It has a BET (BrunauerEmmett-Teller) surface area of $1700 \mathrm{~m}^{2} / \mathrm{g}$ and it consists of micropores of approximately $0.5 \mathrm{~nm}(<$ $0.7 \mathrm{~nm}$ ) and $0.9 \mathrm{~nm}$ and small mesopores of about $3 \mathrm{~nm}$ [37]. Prior to use, AC was sieved through 45 $\mu \mathrm{m}$ sieve and then it was added to the dope solution for preparing the outer layer of the MMM. The concentrations of PES, PVP and AC and the spinning parameters used in the study are specified in Table 1. All polymer solutions were mixed on a roller bench for at least 48 hours, then they were transferred in stainless-steel syringes and left to degas for 24 hours. Afterwards, the syringes were connected to high-pressure syringe pumps and to a double layer spinneret that allows simultaneous co-extrusion of two polymer layers $[17,19]$. Table 2 presents the dimensions of the two spinnerets used for the production of HF-MMM and LF-MMM. Ultrapure water was used as bore forming solution. The air-gap between the spinneret and the coagulation bath was adjusted (Table 1) and a take-up wheel was used for the collection of the produced fibers. The fabricated membranes were washed with demineralized-water and stored for further use. The single layer PES/PVP fiber was produced in the same way and with the same spinneret as the LF-MMM without the outer layer. 
Table 1. Spinning conditions of the dual layer HF-MMM and LF-MMM.

\begin{tabular}{|l|c|c|}
\hline & HF-MMM [17] & LF-MMM [19] \\
\hline Inner layer composition (PES/PVP) & $15 / 10 \mathrm{wt} \%$ & $15 / 7 \mathrm{wt} \%$ \\
\hline Outer layer composition (PES/PVP/AC) & $14 / 1.4 / 60 \mathrm{wt} \%$ & $14 / 1.4 / 60 \mathrm{wt} \%$ \\
\hline Inner layer pumping speed & $0.9 \mathrm{~mL} / \mathrm{min}$ & $0.4 \mathrm{~mL} / \mathrm{min}$ \\
\hline Outer layer pumping speed & $3.2 \mathrm{~mL} / \mathrm{min}$ & $1.6 \mathrm{~mL} / \mathrm{min}$ \\
\hline Bore liquid & $5 \mathrm{wt} \% \mathrm{PVP} / 60 \mathrm{wt} \% \mathrm{NMP} / 35 \mathrm{wt} \%$ ultra-pure water & Ultra-pure water \\
\hline Bore liquid pumping speed & $2.7 \mathrm{~mL} / \mathrm{min}$ & $1.2 \mathrm{~mL} / \mathrm{min}$ \\
\hline Air gap & $3 \mathrm{~cm}$ & $10 \mathrm{~cm}$ \\
\hline Collecting wheel speed & $7 \mathrm{~m} / \mathrm{min}$ & $3.5 \mathrm{~m} / \mathrm{min}$ \\
\hline
\end{tabular}

Table 2. Spinnerets dimensions.

\begin{tabular}{|l|c|c|}
\hline & HF-MMM & LF-MMM \\
\hline Inner diameter needle $(\mathrm{mm})$ & 0.26 & 0.16 \\
\hline Outer diameter needle $(\mathrm{mm})$ & 0.46 & 0.26 \\
\hline Inner diameter first orifice $(\mathrm{mm})$ & 0.66 & 0.46 \\
\hline Outer diameter first orifice $(\mathrm{mm})$ & 0.96 & 0.66 \\
\hline Inner diameter second orifice $(\mathrm{mm})$ & 1.66 & 0.86 \\
\hline
\end{tabular}

\subsection{Membrane characterization}

\subsubsection{Scanning Electron Microscopy (SEM)}

The morphology of the MMM and of the PES/PVP membranes was analyzed by SEM (JEOL, Tokyo, Japan). For the imaging of the cross-sections, the membranes were dried in air and fractured in liquid nitrogen. Prior to SEM imaging, the samples were gold sputtered using the Cressington 108 auto sputter (Cressington Scientific Instruments, Watford, UK).

\subsubsection{Water transport experiments}

The membranes were dried in air and membrane modules having known surface areas were used. Before water transport experiments, the membrane modules $(\mathrm{HF}-\mathrm{MMM} \mathrm{n}=6, \mathrm{LF}-\mathrm{MMM} \mathrm{n}=3$, PES/PVP $n=3$ ) were pre-compacted with ultra-pure water at a trans-membrane pressure (TMP) of 2 Bar for at least 1 hour. Afterwards, the amount of permeated water was measured over time at TMP 
of $0.5,1,1.5$ and 2 Bar. The resulting water permeance was calculated as the slope of the linear fit of the flux $\left(\mathrm{L} /\left(\mathrm{m}^{2} \cdot \mathrm{h}\right)\right)$ versus the TMP (Bar).

\subsubsection{Albumin leakage experiments and Molecular Weight Cut-Off (MWCO)}

The protein leakage for the PES/PVP HF was studied in dialysis simulating expriments in vitro using human blood plasma (obtained by healthy donors in compliance with local ethical guidelines Sanquin, Amsterdam, The Netherlands). Albumin leakage of the HF-MMM and LF-MMM were taken from literature $[17,19]$. The MWCO for all membranes were taken from literature $[17,19,38$, 39], except for PES/PVP, for which the MWCO was not measured.

\subsubsection{Attenuated Total Reflectance-Fourier Transform Infrared Spectroscopy (ATR - FTIR)}

Analysis of the inner surface chemistry of the HF-MMM, LF-MMM and Polysulfone ${ }^{\circledR}$ F60 (Fresenius Medical Care, Bad Homburg, Germany) was performed by ATR-FTIR spectroscopy (Spectrum Two, PerkinElmer) and Spectrum Quant software. All scans were performed at room temperature in triplicate on various parts of the membrane surface at a resolution of $4 \mathrm{~cm}^{-1}$ and were compared to FTIR scans of pure PES and pure PVP materials.

\subsubsection{X-ray photoelectron spectroscopy (XPS)}

XPS analysis of LF-MMM and Polysulfone ${ }^{\circledR}$ F60 membrane surface chemistry was performed using Quantera scanning XPS microprobe (Physical Electronics, Chanhassen, MN, USA) with Al K $\alpha$ excitation radiation $(h v=1486.6 \mathrm{eV})$. The given elemental atomic percentages were averaged from 3 different samples. Data analysis was performed using Compass for XPS control, Multipak v 9.4.0.7. 


\subsection{Mini-modules preparation for hemocompatibility tests}

A predefined surface area of the membranes was inserted into the modules' housings and potted with polyurethane glue by MAT Adsorption Technologies GmbH (Obernburg, Germany). In total 5 different types of modules were prepared: 1. HF-MMM; 2. Cuprophan ${ }^{\circledR}$ F1 (3M Germany GmbH, Wuppertal, Germany); 3. Fresenius Polysulfone ${ }^{\circledR}$ F60; 4. Single layer PES/PVP membrane (PES/PVP); 5. LF-MMM. Module characteristics can be found in the Table 3.

Table 3. Parameters of the membrane modules used in the study.

\begin{tabular}{|c|c|c|c|c|}
\hline Mini-modules materials & $\begin{array}{c}\text { Reference } \\
\text { name }\end{array}$ & $\begin{array}{l}\text { Effective surface } \\
\text { area }\left(\mathrm{cm}^{2}\right)\end{array}$ & $\begin{array}{c}\text { Effective } \\
\text { length }(\mathrm{cm})\end{array}$ & $\begin{array}{l}\text { Number } \\
\text { of fibers }\end{array}$ \\
\hline High-flux MMM - PES/PVP/AC & HF-MMM & 125 & 16 & 36 \\
\hline Cuprophan ${ }^{\circledR}$ F1 - Cellulose & $\mathrm{F} 1$ & 250 & 23 & 174 \\
\hline Polysulfone ${ }^{\circledR}$ F60 - Polysulfone & F60 & 250 & 14 & 284 \\
\hline Single layer membrane - PES/PVP & PES/PVP & 56 & 16 & 36 \\
\hline Low-flux MMM - PES/PVP/AC & LF-MMM & 58 & 16 & 36 \\
\hline
\end{tabular}

\subsection{Hemocompatibility tests}

The hemocompatibility of the tested modules was assessed according to ISO 10993-4 and following standard operation protocols, developed by eXcorLab GmbH (Industrie Center Obernburg, Obernburg, Germany). All experiments were performed on a miniaturized set-up with freshly donated human blood. Blood flow rate was linearly downsized from a membrane surface area used clinically (1.5 $\mathrm{m}^{2}$ and $\mathrm{Qb}_{\mathrm{b}}=300 \mathrm{~mL} / \mathrm{min}$ to $250 \mathrm{~cm}^{2}$ of the respective Cuprophan ${ }^{\circledR}$ mini-module), resulting in an experimental blood flow rate of $5 \mathrm{~mL} / \mathrm{min}$. As a preparation step, all modules were rinsed with saline solution in single pass ( 30 minutes with flow rates of $5 \mathrm{~mL} / \mathrm{min}$ ). At the start of the experiment, saline was replaced by heparinized $(5 \mathrm{IU} / \mathrm{ml})$ human blood $(20 \mathrm{~mL})$, which was recirculated in each circuit for 180 minutes with intraluminal flow $(5 \mathrm{~mL} / \mathrm{min})$. During the experiment, the modules were kept in a water bath at $37^{\circ} \mathrm{C}$, whereas the medium reservoirs were kept at room temperature on a shaker 
to avoid sedimentation of blood cells. Blood samples were collected at time $0,30,120$ and 180 minutes. Total leukocytes (WBC), red blood cells (RBC) and platelets (PLT) counts were measured at each experimental time point. Thrombin-antithrombin III complex (TAT) (as indication of the activation of the coagulation pathway), C5a (as indication for complement activation), and hemolysis were measured at the start and at the end of the experiment (180 minutes). The pressures at the inlet and outlet of the modules were recorded with a multichannel pressure transducer (DPT-6300, Codan pvb Medical GmbH, Forstinning, Germany) and using a laboratory data acquisition system (MSRmanager, HITEC Zang, Herzogenrath, Germany). WBC, RBC and PLT were counted in an ABX Pentra 60 counter (Axon Lab AG, Reichenbach an der Fils, Germany). C5a and TAT were determined by ELISA (DRG Diagnostics, Marburg, Germany; Roche, Mannheim, Germany). Hemolysis was measured by photometry of plasma samples using three wavelengths to correct for (mainly bilirubin) background (OD560nm, OD576nm, OD592nm, photometer UV1650PC, Shimadzu Deutschland $\mathrm{GmbH}$ ). Equation 1 was used to calculate hemolysis (plasma free [Hb]) [40]:

$$
[\mathrm{Hb}]=\frac{\left(2 \mathrm{OD}_{576}-\left(\mathrm{OD}_{560 \mathrm{~nm}}+\mathrm{OD}_{592 \mathrm{~nm}}\right)\right) 99.82+0.36}{1000}
$$

Equation 1

Total $[\mathrm{Hb}]$ at the start of the experiment was measured after complete red cell lysis to express hemolysis as \%. Each minimodule experiment was repeated three times with different donors.

\subsection{Statistics}

All the data are presented as mean \pm SD (standard deviation). Statistical analyses were performed using GraphPad Prism version 5.02 (GraphPad Prism Software, La Jolla, CA, USA). Multiple comparisons between different groups were performed using one-way ANOVA with Bonferroni posthoc test $(\mathrm{p}<0.05)$ in order to determine statistical differences for the pilot experiment (performed to assess the effect of the wall shear rate on C5a and TAT generation) and for the hemocompatibility test. 


\section{Results and discussion}

\subsection{Membrane characterization}

\subsubsection{Membrane morphology}

The morphology of the dual layer HF-MMM, LF-MMM and single layer PES/PVP hollow fiber was investigated using SEM imaging (Figure 1). The LF-MMM [19, 31] (Fig. 1b, e, h, k) is a double layer membrane composed by an inner particle-free PES/PVP layer and an outer layer where AC particles are embedded in the PES/PVP polymer matrix (some of the AC are highlighted with white arrows in Fig. 1 e). In order to guarantee fast diffusion and high adsorption, LF-MMM has a thinner inner layer compared to the outer layer. The two layers are well interconnected and AC particles are well dispersed in the outer layer without aggregation. The membrane has anisotropic morphology, with the selective layer at the inner lumen side of the membrane, in contact with blood, and with finger-like macrovoids throughout the membrane wall (Fig. 1e). The selective layer, responsible for transport selectivity via size exclusion, has lower porosity, with a thickness of approximately $1 \mu \mathrm{m}$ (Fig. 1h) and no visible pores at the lumen surface (Fig. 1k). Given its unique morphology, LF-MMM can guarantee high adsorption of uremic toxins and, thanks to the inner selective layer, good transport properties without leakage of albumin [19] but also barrier properties to avoid transfer of pyrogens to the blood compartment in case of contamination of the dialysate [31]. 


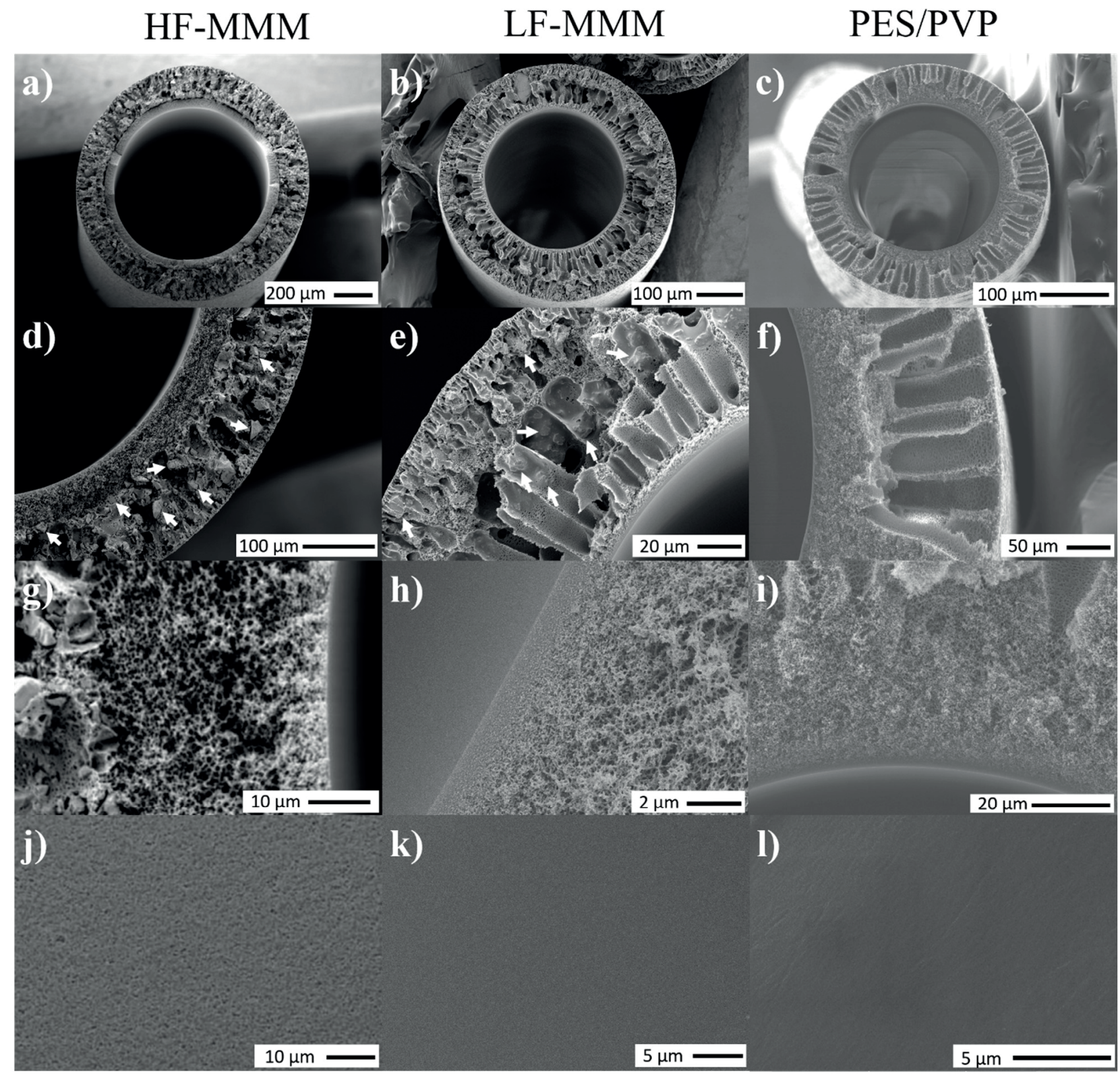

Figure 1. SEM images of the dual layer HF-MMM (a, d, g, j), LF-MMM (b, e, h, k) and PES/PVP membrane (c, f, i, l). Overall cross-sections: a, b, c; magnification of the wall: $d$, e, f; magnification of the inner selective layer: g, h, i; magnification of the inner surface: j, k, l. White arrows highlight some of the AC in Fig. 1d and 1e.

We produced PES/PVP single layer hollow fiber membrane (Fig. 1c, f, i, 1) using the same spinning parameters as for the inner particle-free layer of the LF-MMM. PES/PVP hollow fiber presents similar morphology as the inner layer of the LF-MMM, having finger-like macrovoids (Fig. 1c and 1f), comparable selective layer (approximately $1 \mu \mathrm{m}$ ) at the blood-contacting side of the membrane (Fig. 1i) and no visible pores at the inner surface as LF-MMM (Fig. 11). 
The HF-MMM [17] (Fig. 1a, d, g, j), is a double-layer MMM having, as the LF-MMM, an inner particle-free PES/PVP layer in contact with blood and an outer layer with AC embedded in the polymer matrix (some of the AC are highlighted in Fig. 1d with white arrows). Its selective layer is also present at the lumen side of the fiber and the two layers are well interconnected (Fig. 1d and 1g). Nevertheless, important morphological differences can be observed in comparison to LF-MMM. The HF-MMM [17] has finger-like macrovoids at the outer layer while the inner layer has spongy structure and, since it was manufactured with a different spinneret (Table 2), it has larger dimensions (Fig. 1a and 1d) (Table 4). Another important difference to LF-MMM is that the selective layer of HF-MMM is very thin (Fig, 1g) with visible pores at the lumen surface (Fig. 1j).

The SEM images of Cuprophan ${ }^{\circledR}$ F1 and Polysulfone ${ }^{\circledR}$ F60 (kindly provided by Prof. Dr. J. Vienken) are presented in the Supplementary Information. The dimensions of all membranes are summarized in Table 4.

Table 4. Dimensions of the LF-MMM, HF-MMM [17] and of all the control fibers (Cuprophan ${ }^{\circledR}$ F1, Polysulfone ${ }^{\circledR}$ F60 and PES/PVP hollow fiber).

\begin{tabular}{|l|c|c|}
\hline & Inner diameter $(\boldsymbol{\mu m})$ & Wall thickness $(\boldsymbol{\mu m})$ \\
\hline HF-MMM & 669 & 160 \\
\hline Cuprophan $^{\circledR}$ F1 & 200 & 8 \\
\hline Polysulfone $^{\circledR}$ F60 & 200 & 40 \\
\hline PES/PVP & 301 & 92 \\
\hline LF-MMM & 312 & 105 \\
\hline
\end{tabular}

\subsubsection{Membrane transport experiments}

The ultrafiltration coefficients (KUf) of the produced LF-MMM and PES/PVP membranes were measured and compared to the KUf of HF-MMM, Cuprophan ${ }^{\circledR}$ F1 and Polysulfone ${ }^{\circledR}$ F60 control fibers. The KUf of LF-MMM and PES/PVP is $4 \pm 1 \mathrm{~mL} /\left(\mathrm{m}^{2} \cdot \mathrm{h} \cdot \mathrm{mmHg}\right)$ and $10 \pm 1 \mathrm{~mL} /\left(\mathrm{m}^{2} \cdot \mathrm{h} \cdot \mathrm{mmHg}\right)$, 
respectively, and therefore both can be classified as low-flux dialysis membranes [2]. Also, the stability of operation under transmembrane pressure (TMP) up to 2 Bar was studied and revealed linear fit of the water flux versus pressure. The difference in the KUf of the two fibers could be due to the thinner selective layer and/or to higher surface porosity of the PES/PVP hollow fiber which cannot be appreciated by SEM imaging. It is important to highlight that in our previous work [31] we have shown that the water permeance of the LF-MMM can be improved by washing the membrane with pure ethanol, still maintaining retention properties of the selective layer against plasma proteins (only $0.6 \pm 0.1 \%$ plasma proteins leaked to the dialysate compartment at 4 hours during dialysis experiment compared to the initial amount of proteins in the blood plasma). Cuprophan ${ }^{\circledR} \mathrm{F} 1$ has comparable KUf as the LF-MMM [41], whereas Polysulfone ${ }^{\circledR}$ F60 is a high-flux dialysis membrane $[2,42]$. The HF-MMM has a KUf equal to $76 \pm 12 \mathrm{~mL} /\left(\mathrm{m}^{2} \cdot \mathrm{h} \cdot \mathrm{mmHg}\right)$ and, differently from all the other fibers in this work, has albumin leakage $[2,17]$.

Table 5. Ultrafiltration coefficient (KUf), molecular weight cut-off (MWCO) and albumin leakage of experimental and commercial fibers used for the hemocompatibility test.

\begin{tabular}{|l|c|c|c|}
\hline & $\begin{array}{c}\text { Ultrafiltration coefficient } \\
(\text { KUf })\left(\mathbf{m L} /\left(\mathbf{h} \cdot \mathbf{m}^{2} \cdot \mathbf{m m H g}\right)\right.\end{array}$ & MWCO (KDa) & Albumin leakage \\
\hline HF-MMM & $76 \pm 12[17]$ & $>67[17]$ & YES [17] \\
\hline Cuprophan $^{\circledR}$ F1 & $6[41]$ & $10[38]$ & NO [38] \\
\hline Polysulfone $^{\circledR}$ F60 & $24[42]$ & $30[39]$ & NO [39] \\
\hline PES/PVP & $10 \pm 1$ & Not measured & NO \\
\hline LF-MMM & $4 \pm 1$ & $12[19]$ & NO [19] \\
\hline
\end{tabular}

\subsubsection{Surface characterization}

The membrane lumen surface characterization was performed by means of ATR - FTIR and XPS measurements. Figure 2a compares the ATR - FTIR spectra of LF-MMM, HF-MMM, Polysulfone ${ }^{\circledR}$ F60 membrane, pure PES and pure PVP. The peak at $1677 \mathrm{~cm}^{-1}$, corresponding to the carbonyl groups 
of PVP, has noticeably higher intensity for HF-MMM and LF-MMM in comparison to the Polysulfone ${ }^{\circledR}$ F60, indicating higher amount of PVP at the lumen surface of the two MMM. PES/PVP blends were used for the production of HF-MMM and LF-MMM and therefore the characteristic FTIR peaks of the aromatic bonds of PES, at $1486 \mathrm{~cm}^{-1}$ and $1578 \mathrm{~cm}^{-1}[43,44]$, can be identified in Figure 2a. The F60 is a polysulfone-based membrane, as confirmed also by the peaks at $1488 \mathrm{~cm}^{-1}$ and $1586 \mathrm{~cm}^{-1}$ which are assigned to the aromatic bonds of polysulfone. Moreover, the weak bands at $1386 \mathrm{~cm}^{-1}$ and $1364 \mathrm{~cm}^{-1}$ are typical of methyl groups, exclusively present in the spectrum of polysulfone $[43,44]$. Figure $2 \mathrm{~b}$ shows that nitrogen elemental molar percentage (exclusively part of PVP) is higher at the inner surface of LF-MMM (5\%) compared to Polysulfone ${ }^{\circledR}$ F60 (4\%), consistent to the findings of ATR-FTIR. The carbon content is higher for the F60 compared to LF-MMM. This is due to the presence of the methyl groups in the backbone of polysulfone. Furthermore, as expected, the sulphur content is higher for LF-MMM because of the higher amount of sulfonyl groups in the polymer chain of PES compared to polysulfone.
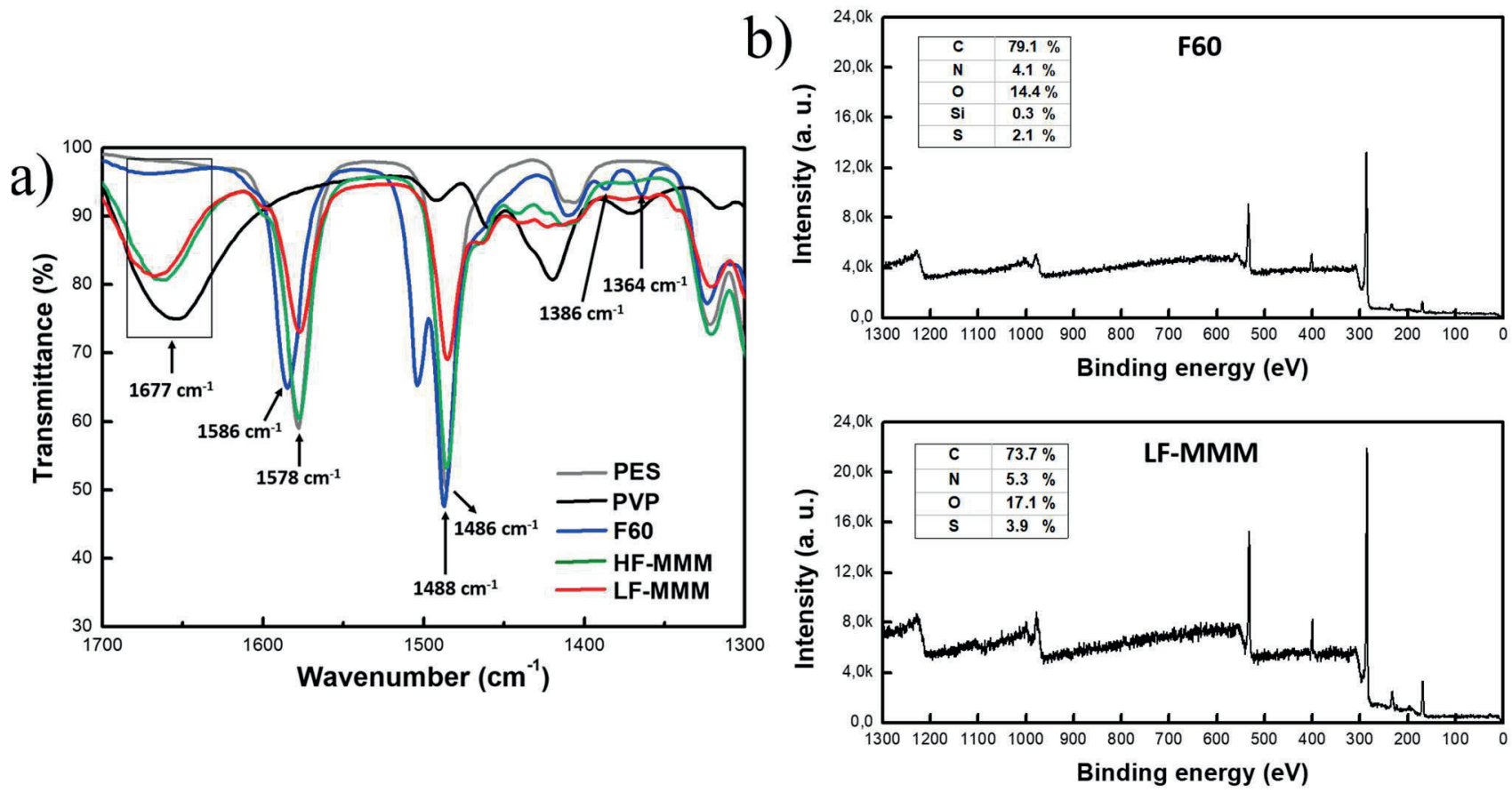

Figure 2. a) ATR-FTIR results of LF-MMM and comparison with HF-MMM, Polysulfone ${ }^{\circledR}$ F60, pure PES and pure PVP material. b) XPS results of LF-MMM and comparison with Polysulfone ${ }^{\circledR}$ F60 commercial fiber. 
For the production of HF-MMM and LF-MMM, we used PES as membrane forming polymer which has thermal stability, mechanical strength, chemical inertness and it can withstand all sterilization techniques [36, 45]. However, its hydrophobic nature favours proteins adhesion, which not only affects membrane performance but can also trigger a series of other reactions such as activation of the coagulation cascade, platelets and red blood cells adhesion with risk of blood clotting and complement and fibrinolysis reactions $[36,45,46]$. The incorporation of hydrophilic additives to the PES membrane guarantees, thanks to the formation of a hydration layer, a reduction of proteins adhesion [36, 46, 47]. Moreover, it is well known that membranes having a combination of hydrophilic and hydrophobic domains, rather than a uniformly hydrophilic or hydrophobic composition, have advantages with respect to biocompatibility [36, 48]. In the membrane-forming dope solutions of HF-MMM and LF-MMM, PES blends with PVP thanks to the strong donoracceptor interaction between $\mathrm{O}=\mathrm{CN}$ functional groups from $\mathrm{PVP}$ and $\mathrm{O}=\mathrm{S}=\mathrm{O}$ from the benzene ring of PES [45]. PVP is a non-ionic, highly polar, amphiphilic, physiologically inert water-soluble polymer which acts as hydrophilic agent [45], thus preventing adsorption and deposition of plasma proteins. Even though blending is the most widely used method in industry, many researchers believe that PVP does not form miscible blends with different grades of PES, and, as a consequence, PVP would be leached out during membrane formation and over time $[45,46]$. In this work, we used PVP K-90 for the production of both MMM. PVP K-90 has been shown to be the most hydrophilic, highly negatively charged, with the lowest fouling behaviour and lowest tendency to leach out of the membrane compared to PVP with lower molecular weights [45]. The ATR-FTIR and XPS results (Figure 2) show that, in our case, PVP is still present at the lumen surface of both MMM and could contribute to low interaction with blood plasma proteins and low membrane fouling. Besides, during the hemocompatibility experiments (Paragraph 3.2.2) the pressure in the inlet and outlet was monitored and there was no indication of changes there indicating no membrane fouling. Moreover, in preliminary 4 hours dialysis simulating experiments, we estimated that the amount of plasma proteins adsorbed on the LF-MMM and PES/PVP was very low ( approximately $3 \%(n=3)$ and 0.5 
$\%(n=3)$ of the starting amount of plasma proteins, respectively). These data were obtained using a miniaturized set-up in diffusion mode $(\mathrm{TMP}=0$ Bar) and applying blood plasma and dialysate flow rates of $1 \mathrm{~mL} / \mathrm{min}$ and $10 \mathrm{~mL} / \mathrm{min}$, respectively. These flow rates are much lower compared to those used in clinic, which are in the range of $300-500 \mathrm{~mL} / \mathrm{min}$ for the blood flow rate and $500-800 \mathrm{~mL} / \mathrm{min}$ for the dialysate flow rate $[2,49]$. The use of such low blood and dialysate experimental flow rates was dictated by the fact that they allowed to mantain the TMP equal to 0 Bar (diffusion mode) with our experimental set-up during the simulating hemodialysis experiment. It is possible that the protein amount missing from the system is due not only to proteins adsorption on the membrane surface but also to possible concentration polarization phenomena at the membrane surface. In fact, at low flow rates, retained blood proteins concentrate preferably at the lumen surface of the membrane instead of being well dispersed in the flowing blood and the measured concentration of the proteins in the blood can be therefore lower.

\subsection{Hemocompatibility study}

\subsubsection{Mini-modules preparation and rheology}

The geometries (inner diameter and wall thickness) of the fibers used in this work are very different from each other and, moreover, the minimodules differ in effective membrane surface area, effective fibers length and number of fibers in the modules (see Table 3). As a result, the rheological parameters for the experimental and reference fibers differ from each other. Table 6 presents the equations used for the calculation of the wall shear rate, Table 7 presents the estimated values of the wall shear rate for all fibers; other rheological parameters are reported in the Supplementary Information. It is well known that geometrical differences influence flow conditions inside the minimodules and, as a consequence, the hemocompatibility results. $[32,45]$. Especially, the wall shear-stress (defined as the tangential force that is exerted by the flowing fluid on the surface of the hollow fiber) is an important parameter affecting the interaction of the blood cells with the membrane; high wall shear-stress can, 
on one hand, reduce protein deposition, but on the other hand, it can induce rupture of red blood cells $[50,51]$. The wall shear-stress linearly correlates with the wall shear rate in case of Newtonian fluids and the blood approaches Newtonian behaviour at shear rates $>2001 / \mathrm{s}[2,52]$. Since, in our study, the wall shear rate in Polysulfone ${ }^{\circledR}$ F60 and Cuprophan ${ }^{\circledR}$ F1 fibers is roughly 5 and 8 times, respectively, higher compared to HF-MMM and that in LF-MMM and PES/PVP is even higher (Table 7), we first performed a pilot experiment (Table 8) to evaluate the effect of wall shear rate on complement activation (C5a generation) and activation of coagulation pathway (TAT generation).

Table 6. Equations used for the calculations of the wall shear rate.

\begin{tabular}{|c|c|}
\hline $\begin{array}{c}\text { Average flow velocity in } \\
\text { one fiber }(\mathrm{cm} / \mathrm{min})\end{array}$ & Wall shear rate $\left.\mathbf{s}^{-1}\right)$ \\
\hline$v=\frac{4 \cdot Q_{B}}{\pi \cdot d_{\text {in }}^{2} \cdot n}$ & $W=\frac{8 \cdot v}{d_{\text {in }}}$ \\
\hline$Q_{B}=$ blood flow \\
$d_{\text {in }}=$ inner diameter of the fiber \\
$n=$ number of fibers \\
\hline
\end{tabular}

Table 7. Flow velocity and wall shear rate values for the different minimodules used in the hemocompatibility experiments.

\begin{tabular}{|l|c|c|}
\hline Membrane/Filter & $\begin{array}{c}\text { Flow velocity in } \\
\text { one fiber (cm/min) }\end{array}$ & $\begin{array}{c}\text { Wall shear } \\
\text { rate }\left(\mathbf{s}^{-1}\right)\end{array}$ \\
\hline HF-MMM & 40 & 79 \\
\hline Cuprophan $^{\circledR}$ F1 & 92 & 613 \\
\hline Polysulfone $^{\circledR}$ F60 & 56 & 373 \\
\hline PES/PVP & 200 & 886 \\
\hline LF-MMM & 182 & 778 \\
\hline
\end{tabular}

We used two different blood flow rates, i. e. $0.5 \mathrm{~mL} / \mathrm{min}$ and $5 \mathrm{~mL} / \mathrm{min}$, to obtain wall shear rates values of $\sim 60 \mathrm{~s}^{-1}$ and $\sim 600 \mathrm{~s}^{-1}$, respectively. As expected, the generation of C5a was higher for 
Cuprophan ${ }^{\circledR}$ F1 minimodules compared to Polysulfone ${ }^{\circledR}$ F60. Importantly, the difference in the wall shear rates applied in this pilot experiment is not large enough to have an impact on the generation of the C5a. The generation of TAT was higher for Polysulfone ${ }^{\circledR}$ F60 compared to Cuprophan ${ }^{\circledR}$ F1, due to the more hydrophobic nature of the polysulfone-based membrane. As for C5a generation, when comparing TAT generation at different wall shear rate values, we could not observe statistically differences depending on the blood flow rate. This indicates that in our set-up differences in fibers geometry, minimodules design and, in particular, in wall shear rate did not affect the C5a generation and generation of TAT (Table 8). Complement (via C5a generation) and coagulation pathway (via TAT-generation) were chosen to study the effect of the rheological parameters (represented by the wall shear rate) on the hemocompatibility profile [53] and, therefore, we decided to proceed with the hemocompatibility experiments of all membranes.

Table 8. Pilot experiment on minimodule rheology and hemocompatibility $(n=3)$.

\begin{tabular}{|l|c|c|c|c|}
\hline \multicolumn{1}{|c|}{ Membrane/filter } & $\begin{array}{c}\text { Blood flow rate } \\
(\mathbf{m L} / \mathbf{m i n})\end{array}$ & $\begin{array}{c}\text { Wall shear rate } \\
\left(\mathbf{s}^{-1}\right)\end{array}$ & $\begin{array}{c}\text { C5a } \\
(\boldsymbol{\mu g} / \mathbf{L})\end{array}$ & $\begin{array}{c}\text { TAT } \\
(\boldsymbol{\mu g} / \mathbf{L})\end{array}$ \\
\hline Cuprophan $^{\circledR} \mathrm{F} 1$ & $0.5 \mathrm{~mL} / \mathrm{min}$ & $60 \mathrm{~s}^{-1}$ & $77 \pm 10$ & $10 \pm 4$ \\
\hline Cuprophan $^{\circledR} \mathrm{F} 1$ & $5 \mathrm{~mL} / \mathrm{min}$ & $600 \mathrm{~s}^{-1}$ & $73 \pm 17$ & $7 \pm 1$ \\
\hline Polysulfone $^{\circledR} \mathrm{F} 60$ & $0.5 \mathrm{~mL} / \mathrm{min}$ & $60 \mathrm{~s}^{-1}$ & $6 \pm 2$ & $39 \pm 24$ \\
\hline Polysulfone $^{\circledR} \mathrm{F} 60$ & $5 \mathrm{~mL} / \mathrm{min}$ & $600 \mathrm{~s}^{-1}$ & $5 \pm 2$ & $25 \pm 12$ \\
\hline
\end{tabular}

\subsubsection{Hemocompatibility tests}

The red blood cells (RBC), white blood cells (WBC) and platelets counts, activation of coagulation, activation of the complement system and hemolysis are important parameters for the assessment of the hemocompatibility profile of medical devices [53]. Here, we investigated these parameters according to ISO 10993-4 after 3 hours of blood contact with all membranes. 
a)

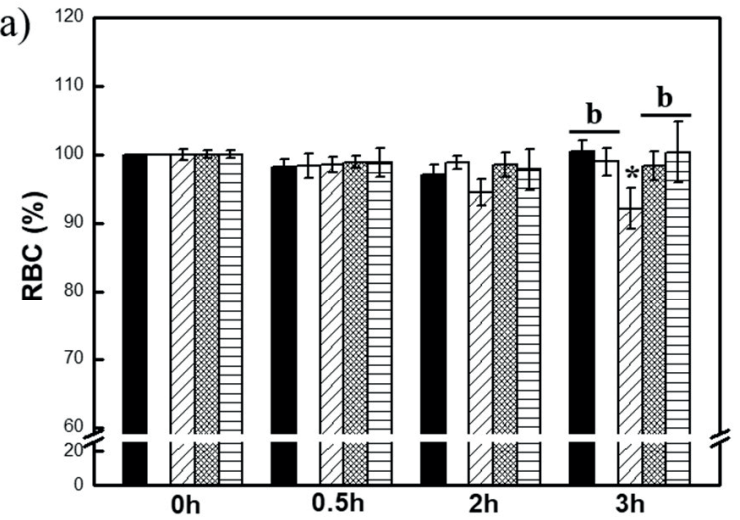

c)

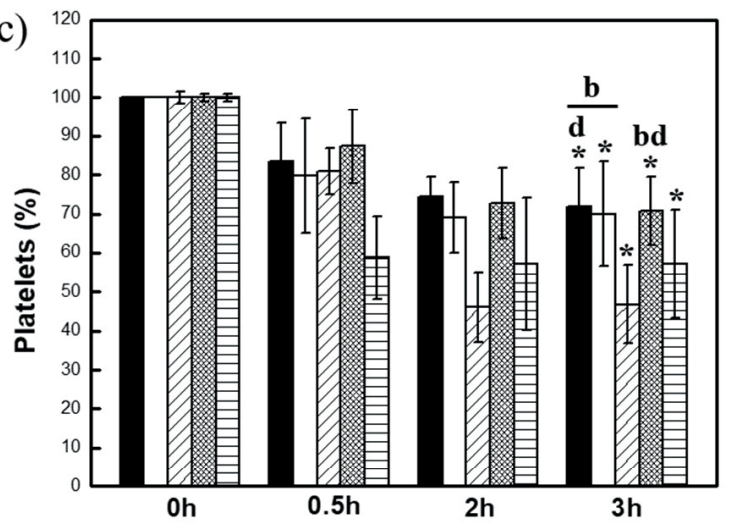

e)

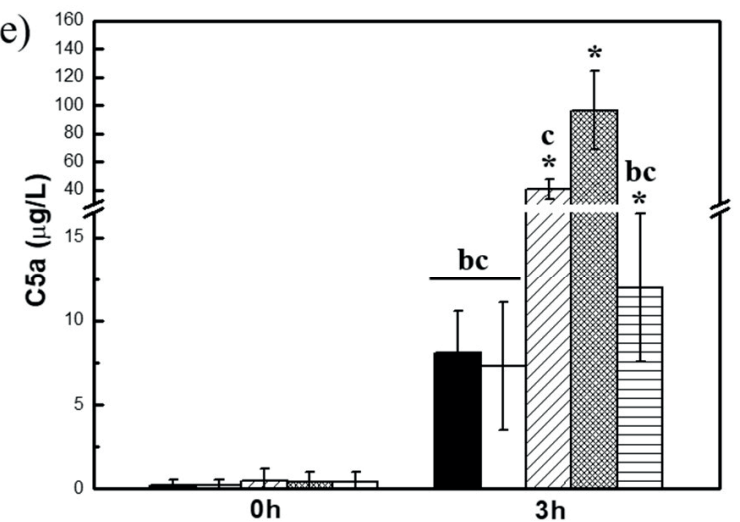

b)

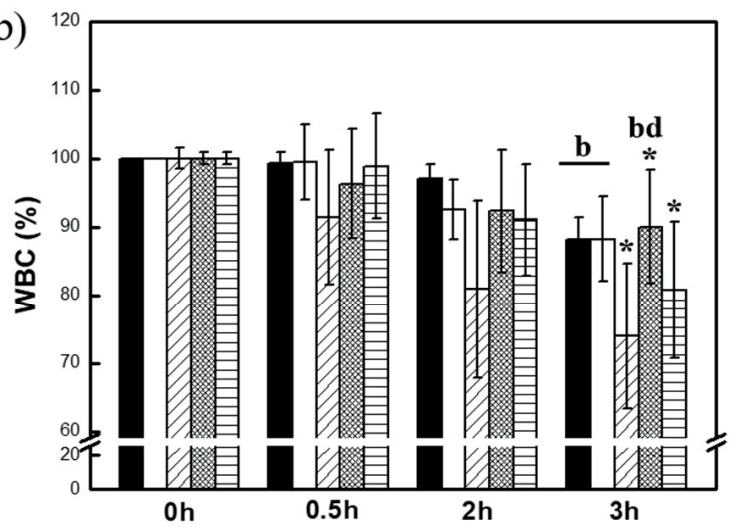

d)

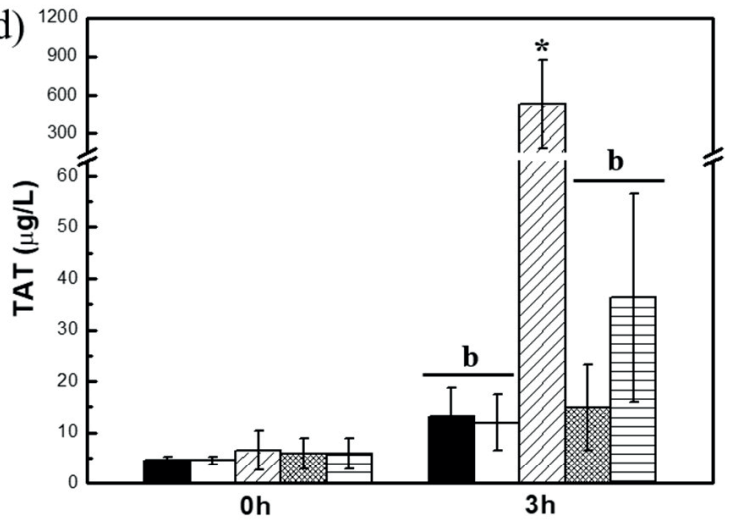

f)

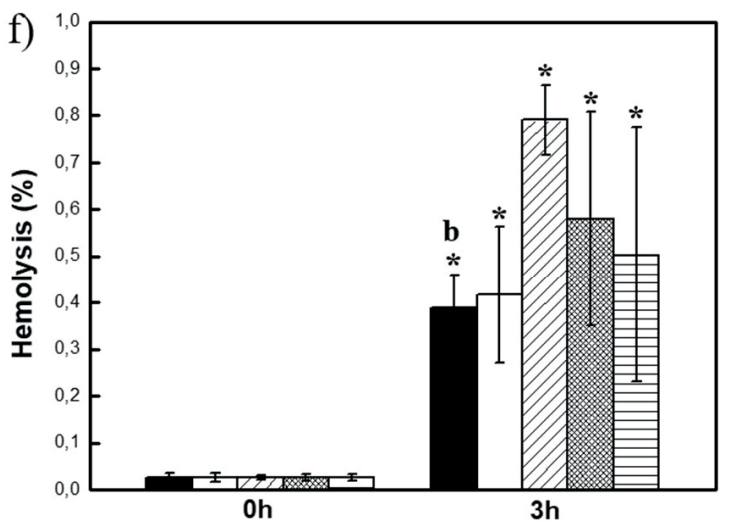

LF-MMM

PES/PVP

7 HF-MMM

Cuprophan F1

Polysulfone F60

Figure 3. Results of hemocompatibility test performed on LF-MMM, PES/PVP, HF-MMM, Cuprophan ${ }^{\circledR}$ F1 and Polysulfone ${ }^{\circledR}$ F60. a) Red blood cells count (RBC, \%); b) White blood cells count (WBC, \%); c) Platelets count (\%); d) Thrombin-antithrombin III complex (TAT) generation $(\mu \mathrm{g} / \mathrm{L})$; e) C5a generation ( $\mu \mathrm{g} / \mathrm{L})$; f) Hemolysis $(\%)$. "b" denotes significant difference versus HF-MMM ( $<<0.05)$; “c" denotes significant difference versus Cuprophan ${ }^{\circledR} F 1(p<0.05)$; " $d$ " denotes significant difference versus Polysulfone ${ }^{\circledR}$ F60 $(\mathrm{p}<0.05)$; “*” denotes significant difference versus the same fiber at time $0 \mathrm{~h}$. 


\section{$R B C$ count and hemolysis}

The damage of erythrocytes can lead to reduced oxygen transport to tissues and organs in vivo and microvesicles derived from erythrocytes can promote thrombus formation [53]. The shear-stress typical of circulatory assist devices affects the mechanical properties of $\mathrm{RBC}$, subsequently impairing their deformability and leading to mechanical fragility [50]. In flowing blood, RBC are stretched to ellipsoids and further stretching imposed by high shear-stress conditions would eventually lead to their rupture [51]. The roughness of the contact surface is also a key factor able to trigger their rupture [54]. In fact, there is a direct correlation of RBC rupture to surface roughness [54]. Hemolysis is the consequence of red blood cell lysis leading to hemoglobin release into the bloodstream, thus an increased concentration of free hemoglobin in the plasma is a direct indicator of erythrocytes destruction $[51,53]$.

In our experiment we observed a significant drop of RBC at 3 hours compared to 0 hours only for the HF-MMM (Figure 3a). Moreover, the level of induced hemolysis of HF-MMM (Figure 3f) was higher compared to the other fibers, consistent with the RBC count results. Since the wall shear rate for HFMMM is considerably lower compared to the other fibers (Table 7), the significant drop of RBC is not expected to be related to high shear-stress conditions. Instead, it is probably due to the roughness of the lumen surface (Fig. 1) which entraps or damages the small RBC. Moreover, based on the FTIR results (Fig. 2a), there is no difference in the amount of PVP at the lumen surface between HF-MMM and LF-MMM, suggesting that the drop of RBC for the HF-MMM cannot be due to chemical adsorption/interaction of the RBC with the lumen surface. Importantly, figure $3 \mathrm{f}$ shows that some hemolysis was observed for all tested membranes at 3 hours (significantly difference compared to 0 hours) but it was below $0.8 \%$ which compares well with the accepted limits for red cell concentrates stored for 42 days in blood banks in Europe (0.8\%) [55] and in USA (1\%) [56]. Moreover, hemolysis less than 5\% is considered as no-toxic according to ASTM F-756-08 standard [57]. Comparable hemolysis results were obtained in earlier studies for flat sheet membranes fabricated with the same blend of PES/PVP as for the PES/PVP hollow fiber and the inner selective layer of the LF-MMM 
used in this work [58]. Frank and co-workers measured the RBC count after two hours of ex-vivo blood recirculation with commercial membranes [59]. In accordance with our results, they did not observe statistical drop in the RBC count when using Polysulfone ${ }^{\circledR}$ and Cuprophan ${ }^{\circledR}$ dialyzers [59]. Also, no significant effects on the RBC were observed by Luo et al. on 17 patients treated with polysulfone membranes [60]. Medina et al. reported destructive influence of HD on RBC and thus evidence of HD contributing to anemia in patients receiving long-term HD with cellulose acetate membrane [61]. The latter results can be related to the poor biocompatibility of cellulose-based mamberanes, resulting in the production of free radicals and reactive oxidant species that can damage RBC and, ultimately, lead to microhemolysis [60].

\section{WBC count}

A drop in WBC count (called leukopenia) is normally observed in clinical HD and indicates adhesion of leukocyte cells to the vessel walls of the lungs in patients and to the dialysis membrane [32, 62]. HD-induced leukopenia is the result of many complex mechanisms cross-talking to each other. On one hand, leukopenia is induced by the activation of the complement system which induces the overexpression of the leukocyte receptors CD11b/CD18 and CD15s. As a consequence, WBC adhere and aggregate to the membrane surface [63]. On the other hand, the adsorption of plasma proteins on the membrane surface triggers the adhesion and aggregation of platelets, which activate the WBC [63]. The latter can result in the up-regulation of adhesive molecules on the cell surface. Moreover, activated cells are more prone to undergo apoptosis, which may ultimately lead to leukopenia [36]. As expected, a decrease of WBC was observed for all the fibers at 3 hours, but statistical difference compared to the WBC at 0 hour was obtained only for HF-MMM, Cuprophan ${ }^{\circledR}$ F1 and Polysulfone ${ }^{\circledR}$ F60 (Figure 3b). For the LF-MMM and PES/PVP fibers, the smaller surface area, the shorter blood residence time in the fibers (Supplementary Information) and the high amount of PVP at the lumen surface (Fig. 2) may explain the lower adsorption of the WBC in comparison to the control fibers. The highest drop of WBC at 3 hours was observed for HF- MMM and Polysulfone ${ }^{\circledR}$ F60 (Figure 3b). 
Given the similar surface lumen chemistry of HF-MMM to LF-MMM (Fig. 2), it is unlikely that the WBC drop is due to the adsorption on the lumen surface; however, the roughness of the inner surface (Fig. 1) might be responsible for entrapment of the WBC, as for the RBC. Besides, the more hydrophobic lumen surface of the Polysulfone ${ }^{\circledR}$ F60 (Fig. 2) is expected to have higher protein adsorption and, consequently, higher WBC deposition. Frank et al. did not observe a significant drop of WBC count after recirculation of human blood in ex-vivo experiments using Polysulfone ${ }^{\circledR}$ and Cuprophan ${ }^{\circledR}$ membranes [59]. Other studies focusing on the inflammatory state of HD patients treated with Polysulfone membranes showed that these hydrophobic membranes can cause neutrophil activation which can accelerate apoptosis $[36,64]$.

\section{Platelets count}

Evaluation of the platelets count after contact with blood is a common parameter to investigate the potential of the material to trigger activation of the coagulation cascade $[65,66]$. High loss of platelets indicates adhesion of platelets on the membrane surface. The first event responsible for the adhesion and aggregation of platelets on the membrane surface is blood protein adhesion [53]. Fibrinogen has been shown to be the key protein in the adhesion process, while other proteins such as von Willebrand factor, immunoglobulins, vitronectin and fibronectin have only supporting effects that may be related to platelet activation [63]. After adhesion, activated platelets accelerate the formation of thrombin [63]. We observed a significant decrease of platelets count for all fibers at 3 hours compared to the start of the experiment (Figure 3c). As for the WBC, the highest drop of platelets occurred for HFMMM and Polysulfone ${ }^{\circledR}$ F60 (Fig. 3c), probably due to the rough and porous lumen surface of the HF-MMM (Fig. 1, which could entrap platelets) and to the hydrophobic character of Polysulfone ${ }^{\circledR}$ F60 (Fig. 2, which represents favourable proteins and, consequently, platelets adsorption sites). It has actually been reported that for Polysulfone ${ }^{\circledR}$ membranes the platelets adhesion is associated and is proportional to proteins adsorption on the membrane surface $[67,68]$ and that platelet counts was lower for Polysulfone ${ }^{\circledR}$ compared to Cuprophan ${ }^{\circledR}$ membranes after blood recirculation [59]. Togo et 
al. [69] measured the plateteles count after blood recirculation using two different types of polysulfone hollow fibers. They found that after 4 hours the platetels count was approximately $40 \%$ lower compared to the start of the experiment. The results of these studies are consistent to our findings [69].

\section{Thrombin generation}

The interaction of plasma proteins with artificial surfaces triggers the coagulation pathway by contact activation which consists of a series of reactions leading to the conversion of factor $\mathrm{X}$ into factor $\mathrm{Xa}$ able to convert prothrombin to thrombin. Thrombin is the final enzyme in the coagulation cascade. It cleaves fibrinogen into fibrin monomers which can then polymerize and cross-link to form a fibrous mesh $[63,66]$. Antithrombin III inhibits thrombin by forming a thrombin-antithrombin III (TAT) complex. This complex reflects a functional state of the coagulation system and can be quantified to measure the amount of thrombin in the blood [53].

In this work, we found statistically higher amount of TAT only for HF-MMM at 3 hours compared to 0 hours (Fig. 3d). This is probably due to the interaction of blood plasma proteins with the AC particles since the selective layer of the HF-MMM has rather high porosity (Fig. 1J) and albumin leakage. Proteins adsorb on the AC due to its hydrophobic nature resulting in the activation of the coagulation pathway, which leads to the conversion of prothrombin into thrombin (detected in this work via the measurement of TAT complex) [53]. The TAT generation by LF-MMM is not statistically different to the amount of TAT at the start of the experiment and to PES/PVP fiber at 3 hours (Fig. 3d). This finding highlights that the lumen surface of the LF-MMM does not induce coagulation cascade activation itself and indicates that the inner layer of the LF-MMM could act as safe barrier to avoid the contact of blood components with the AC present in the outer layer. Frank et al. observed that Cuprophan ${ }^{\circledR}$ and Polysulfone ${ }^{\circledR}$ membranes induce TAT generation in the same order 
of magnitude as the values obtained in our case for the commercial fibers, thus confirming the validity of our results [59].

\section{Complement system activation}

The complement system is a key component of the innate immune system, which can be activated in case of invading microorganisms and when blood contacts with artificial surfaces [53, 70]. Complement activation during HD occurs via the alternative pathway $[53,66,71]$. The final step in the complement cascade is the formation of the membrane attack complex (MAC, C5b9) which leads to cell lysis [63]. Besides the formation of the MAC complex, the anaphylotoxins C3a and C5a are generated. $\mathrm{C} 3 \mathrm{a}$ and $\mathrm{C} 5 \mathrm{a}$ are potent agents capable of producing intense inflammation reactions as vascular smooth muscle contraction [66] and migration of activated neutrophils to the lungs [71]. This latter effect results in mild pulmonary dysfunction during hemodialysis, particularly if dialysate containing acetate is used [71].

Here, we evaluated the activation of the complement system by measuring the generation of C5a fragments in the blood [48, 53] (Fig. 3e). We observed an increase of C5a generation at 3 hours for all the fibers compared to 0 hours; importantly, the LF-MMM induces low activation of the complement system similarly to that induced by Polysulfone ${ }^{\circledR}$ F60 and PES/PVP (Fig. 3e). As expected, the generation of $\mathrm{C} 5 \mathrm{a}$ with Cuprophan ${ }^{\circledR} \mathrm{F} 1$ is statistically higher compared to all other fibers (Fig. 3e). This well-known property of cellulose-based membranes to activate the complement system is due to the presence of free hydroxyl groups [36, 66]. Similarly, in in vitro blood compatibility experiments using flat sheet membranes we observed an increased activation of the complement system with cellulose membranes compared to PES and PES/PVP membranes [58]. Moreover, Frank et al. reported that the $\mathrm{C} 5 \mathrm{a}$ concentration during ex vivo recirculation with Cuprophan ${ }^{\circledR}$ was increased at a high extent, while only low level of C5a activation was detectable following recirculation with the Polysulfone ${ }^{\circledR}$ [59]. Also, Uhlenbush-Körwer et al. observed higher C5a generation for Cuprophan ${ }^{\circledR}$ membranes compared to Polysulfone ${ }^{\circledR}$ membrane [72]. 
The generation of C5a for the HF-MMM at $3 \mathrm{~h}$ is significantly different to that at $0 \mathrm{~h}$. Due to the high porosity of the selective layer of HF-MMM (MWCO > 66.5 KDa) [17], C5a ( $13 \mathrm{KDa})$ could reach the $\mathrm{AC}$ on the outer surface. We do not know if the amount of C5a measured for the HF-MMM derives only from C5a generation or if it is the result of generation and adsorption on the AC; further experiments should elucidate this point. In other studies it has been observed that the concentration of the low molecular weight protein $\mathrm{C} 5 \mathrm{a}$ in the blood represents the net result of the simultaneous processes of generation and any dialytic removal (diffusion, convection, adsorption) that may occur $[48,73,74]$. For example, PAN (polyacrylonitrile) membranes have high adsorptive capacity for complement fragments. Thus, the net effect is only a modest elevation of these products systemicly $[48,66]$. In another work it was observed that the peak C3a concentration obtained with large pore cellulose acetate membrane was significantly less than that obtained with its smaller pore counterpart and was not significantly different from that of low-flux polysulfone [48].

\section{Overall hemocompatibility assessment}

In summary, LF-MMM has hemocompatibility profile similar to that of fibers currently used in clinics. The PVP-enriched lumen surface which prevents proteins adsorption, the smooth lumen surface and, especially, the lumen selective layer which protects the blood to contact the AC in the outer layer, are responsible for the good hemocompatibility of the LF-MMM. Moreover, the morphology of the LF-MMM guarantees high removal of uremic toxins, especially protein-blood toxins $[19,31]$. In the case of HF-MMM, there is activation of the coagulation pathway due to plasma protein leakage across the selective inner layer and the interaction with the AC. Furthermore, the reduction of red and white cells and platelets observed for the HF-MMM is attributable to the high surface roughness of its inner layer. 


\section{Conclusion and outlook}

In this work, we studied the hemocompatibility profile of two mixed matrix membranes (MMM), one with low flux properties and no albumin leakage (LF-MMM) and another characterized by high flux and albumin leakage (HF-MMM). The surface characterization of the lumen of the LF-MMM shows that the high amount of polyvinylpyrrolidone and the smoothness of the lumen blood-contacting surface limit adsorption of blood proteins and adsorption and/or entrapment of blood cells. Importantly, we consider that the good hemocompatibility performance of LF-MMM compared to the HF-MMM is also due to the protective selective layer of the LF-MMM which represents a safe barrier to prevent leakage of plasma proteins and contact of blood with activated carbon.

As a next step, we aim to upscale the production of the LF-MMM and modules and to test its performance in terms of toxins removal and hemocompatibility profile using full blood and dialysate flow rates usually applied in clinics.

\section{Acknowledgments}

This work was supported by the EU Marie Curie ITN Project BIOART (grant no. 316690, EU-FP7PEOPLE-ITN-2012) and by the European Union's Horizon 2020 research and innovation program TheLink (Marie Skłodowska-Curie grant agreement no. 642890, http://thelink-project.eu/). 


\section{References}

[1] M. Storr, R.A. Ward, Membrane innovation: closer to native kidneys, Nephrology, Dialysis, Transplantation 33(suppl. 3) (2018) 22-27.

[2] C. Ronco, W.R. Clark, Haemodialysis membranes, Nature Reviews Nephrology 14(6) (2018) 394-410.

[3] W.R. Clark, F. Ferrari, C. Ronco, Chapter 189 - Sorbents: From basic structure to clinical application, in: C. Ronco, R. Bellomo, J.A. Kellum, Z. Ricci (Eds.), Critical Care Nephrology (Third Edition) (2019) 11371154

[4] H.R. Wang, J. Pan, A.D. Shang, Y.Q. Lu, Time-dependent haemoperfusion after acute paraquat poisoning, Scientific Reports 7(1) (2017) 2239-2239.

[5] T. Shimizu, T. Miyake, M. Tani, History and current status of polymyxin B-immobilized fiber column for treatment of severe sepsis and septic shock, Annals of Gastroenterological Surgery 1(2) (2017) 105-113.

[6] T. Iba, L. Fowler, Is polymyxin B-immobilized fiber column ineffective for septic shock? A discussion on the press release for EUPHRATES trial, Journal of Intensive Care 5 (2017) 40-40.

[7] X. Yang, S. Xin, Y. Zhang, T. Li, Early hemoperfusion for emergency treatment of carbamazepine poisoning, The American Journal of Emergency Medicine 36(6) (2018) 926-930.

[8] K. Kogelmann, D. Jarczak, M. Scheller, M. Druner, Hemoadsorption by CytoSorb in septic patients: a case series, Critical Care 21(1) (2017) 74.

[9] H. Yatzidis, A convenient hemoperfusion microapparatus over charcoal for the treatment of endogenous and exogenous intoxification: Its use as an effective artificial kidney, Proceedigns of the European Dialysis and Transplantation Association 1 (1964) 83-87.

[10] S.V. Mikhalovsky, Emerging technologies in extracorporeal treatment: focus on adsorption, Perfusion 18 (Suppl. 1) (2003) 47-54.

[11] E. Erturk, M. Haberal, E. Piskin, Towards the commercialization of a haemoperfusio column. Part I: Selection of activated carbon, Clinical Materials 2(1) (1987) 55-65.

[12] P.K. Tyagi, J.F. Winchester, D.A. Feinfeld, Extracorporeal removal of toxins, Kidney International 74(10) (2008) 1231-1233.

[13] J.F. Winchester, C. Ronco, Sorbent hemoperfusion in end-stage renal disease: An in-depth review, Advances in Chronic Kidney Disease 9(1) (2002) 19-25. 
[14] N. Cai, Q. Li, J. Zhang, T. Xu, W. Zhao, J. Yang, L. Zhang, Antifouling zwitterionic hydrogel coating improves hemocompatibility of activated carbon hemoadsorbent, Journal of Colloid and Interface Science 503 (2017) 168-177.

[15] M. Sternkopf, S. Thoroe-Boveleth, T. Beck, K. Oleschko, A. Erlenkotter, U. Tschulena, S. Steppan, T. Speer, C. Goettsch, V. Jankowski, J. Jankowski, H. Noels, A bifunctional adsorber particle for the removal of hydrophobic uremic toxins from whole blood of renal failure patients, Toxins 11(7) (2019).

[16] C.A. Howell, S.R. Sandeman, Y. Zheng, S.V. Mikhalovsky, V.G. Nikolaev, L.A. Sakhno, E.A. Snezhkova, New dextran coated activated carbons for medical use, Carbon 97 (2016) 134-146.

[17] M.S. Tijink, M. Wester, G. Glorieux, K.G. Gerritsen, J. Sun, P.C. Swart, Z. Borneman, M. Wessling, R. Vanholder, J.A. Joles, D. Stamatialis, Mixed matrix hollow fiber membranes for removal of protein-bound toxins from human plasma, Biomaterials 34(32) (2013) 7819-28.

[18] M.S.L. Tijink, M. Wester, J. Sun, A. Saris, L.A.M. Bolhuis-Versteeg, S. Saiful, J.A. Joles, Z. Borneman, M. Wessling, D.F. Stamatialis, A novel approach for blood purification: Mixed-matrix membranes combining diffusion and adsorption in one step, Acta Biomaterialia 8(6) (2012) 2279-2287.

[19] D. Pavlenko, E. van Geffen, M.J. van Steenbergen, G. Glorieux, R. Vanholder, K.G.F. Gerritsen, D. Stamatialis, New low-flux mixed matrix membranes that offer superior removal of protein-bound toxins from human plasma, Scientific Reports 6 (2016) 34429.

[20] D.C. Dinh, N.S. Recht, T.H. Hostetter, T.W. Meyer, Coated carbon hemoperfusion provides limited clearance of protein-bound solutes, Artificial Organs 32(9) (2008) 717-724.

[21] S.R. Ash, Sorbents in treatment of uremia: a short history and a great future, Seminars in Dialysis 22(6) (2009) 615-22.

[22] B. Bammens, P. Evenepoel, H. Keuleers, K. Verbeke, Y. Vanrenterghem, Free serum concentrations of the protein-bound retention solute p-cresol predict mortality in hemodialysis patients, Kidney International 69(6) (2006) 1081-7.

[23] S. Ito, M. Osaka, Y. Higuchi, F. Nishijima, H. Ishii, M. Yoshida, Indoxyl sulfate induces leukocyteendothelial interactions through up-regulation of E-selectin, Journal of Biological Chemistry 285(50) (2010) 38869-38875.

[24] A. Adijiang, S. Goto, S. Uramoto, F. Nishijima, T. Niwa, Indoxyl sulphate promotes aortic calcification with expression of osteoblast-specific proteins in hypertensive rats, Nephrology, Dialysis, Transplantation 23(6) (2008) 1892-1901. 
[25] C.J. Lin, C.F. Pan, H.L. Liu, C.K. Chuang, T. Jayakumar, T.J. Wang, H.H. Chen, C.J. Wu, The role of protein-bound uremic toxins on peripheral artery disease and vascular access failure in patients on hemodialysis, Atherosclerosis 225(1) (2012) 173-9.

[26] I.W. Wu, K.H. Hsu, C.C. Lee, C.Y. Sun, H.J. Hsu, C.J. Tsai, C.Y. Tzen, Y.C. Wang, C.Y. Lin, M.S. Wu, p-Cresyl sulphate and indoxyl sulphate predict progression of chronic kidney disease, Nephrology, Dialysis, Transplantation 26(3) (2011) 938-947.

[27] Y. Itoh, A. Ezawa, K. Kikuchi, Y. Tsuruta, T. Niwa, Protein-bound uremic toxins in hemodialysis patients measured by liquid chromatography/tandem mass spectrometry and their effects on endothelial ROS production, Analytical and Bioanalytical Chemistry 403(7) (2012) 1841-1850.

[28] A. Adijiang, Y. Higuchi, F. Nishijima, H. Shimizu, T. Niwa, Indoxyl sulfate, a uremic toxin, promotes cell senescence in aorta of hypertensive rats, Biochemical and Biophysical Research Communications 399(4) (2010) 637-641.

[29] S. Lekawanvijit, A. Adrahtas, D.J. Kelly, A.R. Kompa, B.H. Wang, H. Krum, Does indoxyl sulfate, a uraemic toxin, have direct effects on cardiac fibroblasts and myocytes?, European Heart Journal 31(14) (2010) 1771-1779.

[30] S. Eloot, W. Van Biesen, R. Vanholder, A Sad but Forgotten Truth: The Story of Slow-Moving Solutes in Fast Hemodialysis, Seminars in Dialysis 25(5) (2012) 505-509.

[31] I. Geremia, R. Bansal, D. Stamatialis, In vitro assessment of mixed matrix hemodialysis membrane for achieving endotoxin-free dialysate combined with high removal of uremic toxins from human plasma, Acta Biomater 90 (2019) 100-111.

[32] J. Mulvihill, J.P. Cazenave, J.P. Mazzucotelli, T. Crost, C. Collier, J.L. Renaux, C. Pusineri, Minimodule dialyser for quantitative ex vivo evaluation of membrane haemocompatibility in humans: comparison of acrylonitrile copolymer, cuprophan and polysulphone hollow fibres, Biomaterials 13(8) (1992) 527-536.

[33] T. Bouré, R. Vanholder, Which dialyser membrane to choose?, Nephrology, Dialysis, Transplantation 19(2) (2004) 293-296.

[34] S.K. Bowry, Dialysis membranes today, The International Journal of Artificial Organs 25(5) (2002) 447460.

[35] N.A. Hoenich, C. Woffindin, A. Brennan, P.J. Cox, J.N. Matthews, M. Goldfinch, A comparison of three brands of polysulfone membranes, Journal of the American Society of Nephrology 7(6) (1996) 871-876. 
[36] M. Kohlova, C.G. Amorim, A. Araujo, A. Santos-Silva, P. Solich, M. Montenegro, The biocompatibility and bioactivity of hemodialysis membranes: their impact in end-stage renal disease, Journal of Artificial Organs 22(1) (2019) 14-28.

[37] D. Pavlenko, D. Giasafaki, G. Charalambopoulou, E. van Geffen, K.G.F. Gerritsen, T. Steriotis, D. Stamatialis, Carbon adsorbents with dual porosity for efficient removal of uremic toxins and cytokines from human plasma, Scientific Reports 7(1) (2017) 14914.

[38] V.E.S. Hjerten, Simple micro method for concentration and desalting utilizing a hollow fiber, with special reference to capillary electrophoresis, 1999.

[39] B. Rasuli, N. Tabkhi, E. Kalantar, V. Zarezade, M.B. shiran, Influence of ultrasound on the clearance of toxic substances from Polysulfone hollow fiber membrane used in a dialyzer filter, Polish Journal of Medical Physics and Engineering 24 (2018) 91.

[40] C.M. Cripps, Rapid method for the estimation of plasma haemoglobin levels, Journal of Clinical Pathology 21(1) (1968) 110-112.

[41] R.E.N.P.R. Slowiaczek, K. Schindhelm, B. Milthorpe, Cell Separation Device, 1998.

[42] T. Masaki, J. Gilson, J.K. Leypoldt, A.K. Cheung, Effect of permeability on indices of haemodialysis membrane biocompatibility, Nephrology, Dialysis, Transplantation 14(5) (1999) 1176-81.

[43] S. Belfer, R. Fainchtain, Y. Purinson, O. Kedem, Surface characterization by FTIR-ATR spectroscopy of polyethersulfone membranes-unmodified, modified and protein fouled, Journal of Membrane Science 172(1) (2000) 113-124.

[44] M.T. Tsehaye, S. Velizarov, B. Van der Bruggen, Stability of polyethersulfone membranes to oxidative agents: A review, Polymer Degradation and Stability 157 (2018) 15-33.

[45] M. Irfan, A. Idris, Overview of PES biocompatible/hemodialysis membranes: PES-blood interactions and modification techniques, Materials Science and Engineering: C 56 (2015) 574-592.

[46] F. Ran, X. Niu, H. Song, C. Cheng, W. Zhao, S. Nie, L. Wang, A. Yang, S. Sun, C. Zhao, Toward a highly hemocompatible membrane for blood purification via a physical blend of miscible comb-like amphiphilic copolymers, Biomaterials Science 2(4) (2014) 538-547.

[47] J. Li, F. Liu, Y. Qin, J. He, Z. Xiong, G. Deng, Q. Li, A novel natural hirudin facilitated anti-clotting polylactide membrane via hydrogen bonding interaction, Journal of Membrane Science 523 (2017) 505-514.

[48] W.R. Clark, R.J. Hamburger, M.J. Lysaght, Effect of membrane composition and structure on solute removal and biocompatibility in hemodialysis, Kidney International 56(6) (1999) 2005-15. 
[49] N. Hoenich, Blood flow during hemodialysis, Seminars in Dialysis 11(4) (1998) 237-241.

[50] J.T. Horobin, S. Sabapathy, M.J. Simmonds, Repetitive supra-physiological shear stress impairs red blood cell deformability and induces hemolysis, Artificial Organs 41(11) (2017) 1017-1025.

[51] H.D. Polaschegg, Red blood cell damage from extracorporeal circulation in hemodialysis, Seminars in Dialysis 22(5) (2009) 524-531.

[52] C. Ronco, P.M. Ghezzi, A. Brendolan, C. Crepaldi, G. La Greca, The haemodialysis system: basic mechanisms of water and solute transport in extracorporeal renal replacement therapies, Nephrology, Dialysis, Transplantation 13 (Suppl. 6) (1998) 3-9.

[53] M. Weber, H. Steinle, S. Golombek, L. Hann, C. Schlensak, H.P. Wendel, M. Avci-Adali, Bloodcontacting biomaterials: in vitro evaluation of the hemocompatibility, Frontiers in Bioengineering and Biotechnology 6 (2018) 99.

[54] M. Umezu, T. Yamada, H. Fujimasu, T. Fujimoto, M. Ranawake, A. Nogawa, T. Kijima, Effects of surface roughness on mechanical hemolysis, Artificial Organs 20(6) (1996) 575-578.

[55] European Committee (Partial Agreement) on Blood Transfusion (CD-P-TS), Guide to the preparation, use and quality assurance of blood components, (19th Edition) (2017).

[56] Public Workshop: Pre-clinical evaluation of red blood cells for transfusion (2016).

[57] M. Tijink, J. Janssen, M. Timmer, J. Austen, Y. Aldenhoff, J. Kooman, L. Koole, J. Damoiseaux, R. van Oerle, Y. Henskens, D. Stamatialis, Development of novel membranes for blood purification therapies based on copolymers of N-vinylpyrrolidone and n-butylmethacrylate, Journal of Materials Chemistry B 1(44) (2013) 6066-6077.

[58] O. ter Beek, D. Pavlenko, M. Suck, S. Helfrich, L. Bolhuis-Versteeg, D. Snisarenko, C. Causserand, P. Bacchin, P. Aimar, R. van Oerle, R. Wetzels, P. Verhezen, Y. Henskens, D. Stamatialis, New membranes based on polyethersulfone - SlipSkin ${ }^{\mathrm{TM}}$ polymer blends with low fouling and high blood compatibility, Separation and Purification Technology 225 (2019) 60-73.

[59] R.D. Frank, J. Weber, H. Dresbach, H. Thelen, C. Weiss, J. Floege, Role of contact system activation in hemodialyzer-induced thrombogenicity, Kidney International 60(5) (2001) 1972-1981.

[60] J.F. Luo, J.H. Li, J.J. Nie, P.P. Li, H.D. Zhang, Y.J. Ma, Effect of hemodialysis on the red blood cell life span in patients with end-stage kidney disease, Therapeutic Apheresis and Dialysis 23(4) (2019) 336-340.

[61] A. Medina, C. Ellis, M.D. Levitt, Use of alveolar carbon monoxide measurements to assess red blood cell survival in hemodialysis patients, American Journal of Hematology 46(2) (1994) 91-94. 
[62] M.P. Grooteman, J.C. Bos, A.J. van Houte, J. van Limbeek, M. Schoorl, M.J. Nubé, Mechanisms of intradialyser granulocyte activation: a sequential dialyser elution study, Nephrology Dialysis Transplantation 12(3) (1997) 492-499.

[63] L. Bartolo, E. Curcio, E. Drioli, Membrane systems: For bioartificial organs and regenerative medicine, De Gruyter (2017).

[64] R.A. Ward, R. Ouseph, K.R. McLeish, Effects of high-flux hemodialysis on oxidant stress, Kidney International 63(1) (2003) 353-359.

[65] K. Kokubo, Y. Kurihara, K. Kobayashi, H. Tsukao, H. Kobayashi, Evaluation of the biocompatibility of dialysis membranes, Blood Purification 40(4) (2015) 293-297.

[66] R.M. Hakim, Clinical implications of hemodialysis membrane biocompatibility, Kidney International 44(3) (1993) 484-494.

[67] K. Namekawa, M. Fukuda, M. Matsuda, Y. Yagi, K. Yamamoto, K. Sakai, Nanotechnological characterization of human serum albumin adsorption on wet synthetic polymer dialysis membrane surfaces, American Society for Artificial Internal Organs Journal 55(3) (2009) 236-242.

[68] Y. Koga, H. Fujieda, H. Meguro, Y. Ueno, T. Aoki, K. Miwa, M. Kainoh, Biocompatibility of polysulfone hemodialysis membranes and its mechanisms: involvement of fibrinogen and its integrin receptors in activation of platelets and neutrophils, Artificial Organs 42(9) (2018) 246-258.

[69] K. Togo, M. Yamamoto, M. Imai, K. Akiyama, A.C. Yamashita, Comparison of blood compatibility in various membrane materials for continuous renal replacement therapy, Therapeutic Apheresis and Dialysis 24(1) (2020) 26-33.

[70] J.R. Dunkelberger, W.C. Song, Complement and its role in innate and adaptive immune responses, Cell Research 20(1) (2010) 34-50.

[71] J.F. Winchester, C. Ronco, J.A. Brady, L.D. Cowgill, J. Salsberg, E. Yousha, M. Choquette, R. Albright, J. Clemmer, V. Davankov, M. Tsyurupa, L. Pavlova, M. Pavlov, G. Cohen, W. Horl, F. Gotch, N.W. Levin, The next step from high-flux dialysis: Application of sorbent technology, Blood Purification 20(1) (2002) 8186.

[72] K. Uhlenbusch, S. Bonnie, A. Grassmann, J. Vienken, Understanding Membranes and Dialysers, Pabst Science Publisher (2003).

[73] F. Poppelaars, B. Faria, M. Gaya da Costa, C.F.M. Franssen, W.J. van Son, S.P. Berger, M.R. Daha, M.A. Seelen, The Complement System in Dialysis: A Forgotten Story?, Frontiers in Immunology 9 (2018) 71. 
[74] J. Mares, V. Thongboonkerd, Z. Tuma, J. Moravec, M. Matejovic, Specific adsorption of some complement activation proteins to polysulfone dialysis membranes during hemodialysis, Kidney International 76(4) (2009) 404-413. 


\section{Supplementary Information}

\section{Membrane morphology of Polysulfone ${ }^{\circledR}$ F60 and Cuprophan ${ }^{\circledR}$ F1}

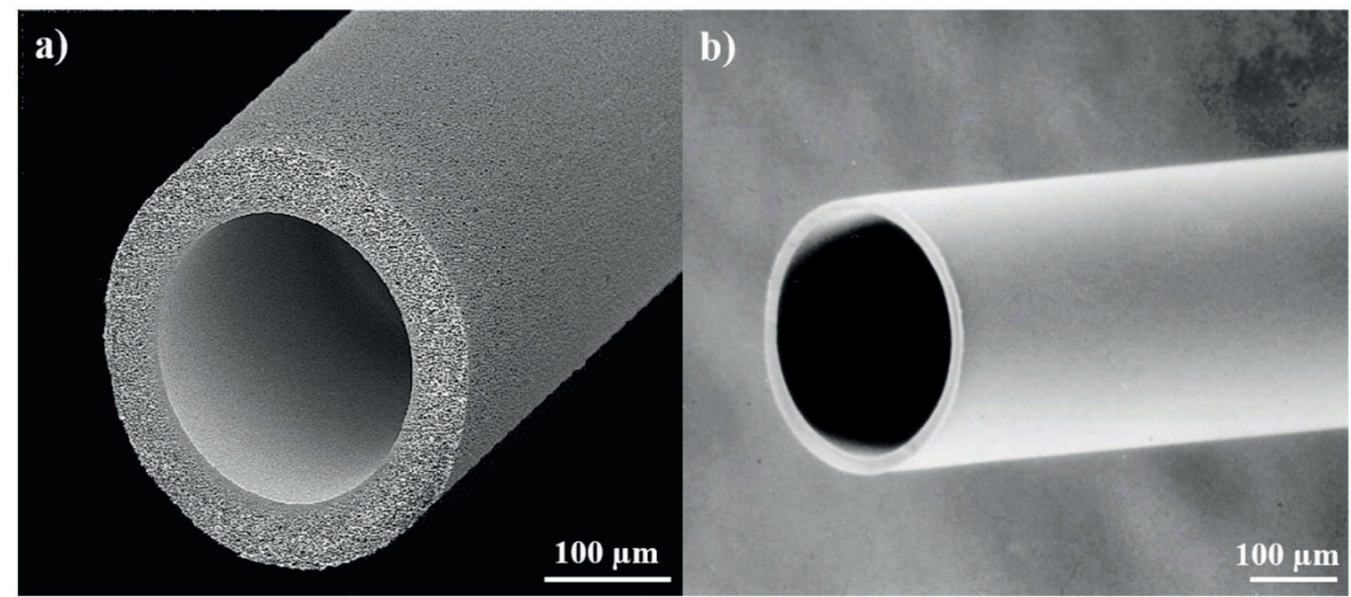

Figure S1. SEM images of (a) Polysulfone ${ }^{\circledR}$ F60 and (b) Cuprophan ${ }^{\circledR}$ F1 (kindly provided by Prof. Dr. J. Vienken)

Polysulfone ${ }^{\circledR}$ F60 membrane (F60) is characterized by a spongy structure with the selective layer at the inner lumen side of the hollow fiber and no visible pores. Cuprophan ${ }^{\circledR} \mathrm{F} 1$ hollow fiber has isotropic structure with no visible pores and with thinner membrane wall compared to the Polysulfone ${ }^{\circledR}$ F60. The dimensions of the two fibers are reported in Table 4 of Chapter 3.

\section{Rheological parameters}

Table S1. Equations used for the calculations of rheological parameters.

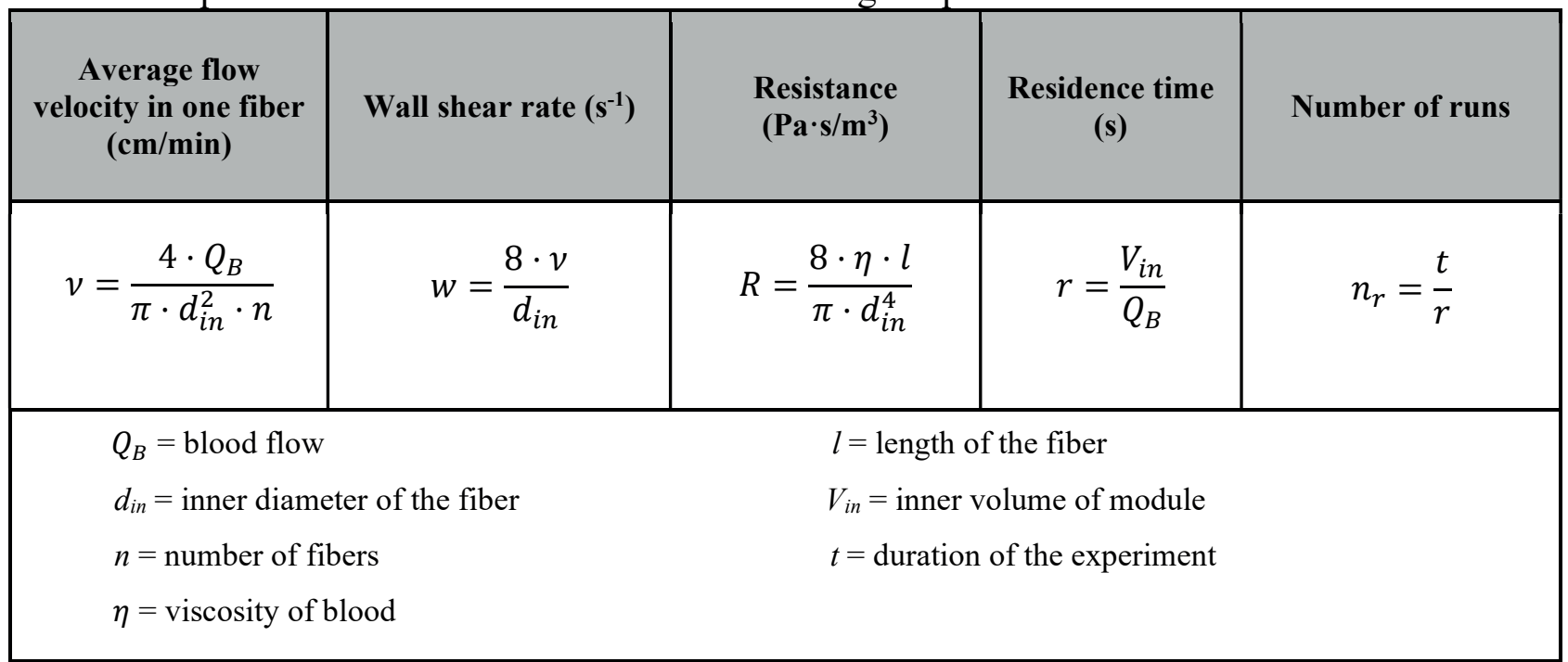


Average flow velocity refers to blood unit velocity inside a module and depends on the numbers of fibers. Wall shear rate affects the blood cells and proteins interactions with the membrane. Resistance depends on the effective length and inner diameter of the fibers, while longer residence time may enhance blood activation due to slower flow rate. The number of runs refers to the total blood volume, the blood flow rate during experiment and the duration of the experiment.

Table S2. Rheological parameters for the different minimodules used in the hemocompatibility experiments.

\begin{tabular}{|l|c|c|c|c|c|}
\hline \multicolumn{1}{|c|}{ Membrane/Filter } & $\begin{array}{c}\text { Flow velocity in } \\
\text { one fiber }(\mathbf{c m} / \mathbf{m i n})\end{array}$ & $\begin{array}{c}\text { Wall shear } \\
\text { rate }\left(\mathbf{s}^{-1}\right)\end{array}$ & $\begin{array}{c}\text { Resistance } \\
\left.(\mathbf{( P a} \cdot \mathbf{s}) / \mathbf{m}^{3}\right)\end{array}$ & $\begin{array}{c}\text { Residence } \\
\text { time (s) }\end{array}$ & $\begin{array}{c}\text { Number of } \\
\text { runs }\end{array}$ \\
\hline HF-MMM & 40 & 79 & $8 \cdot 10^{9}$ & 25 & 439 \\
\hline Cuprophan ${ }^{\circledR}$ F1 & 92 & 613 & $1 \cdot 10^{12}$ & 16 & 692 \\
\hline Polysulfone ${ }^{\circledR}$ F60 & 56 & 373 & $6 \cdot 10^{11}$ & 16 \\
\hline PES/PVP & 200 & 886 & $1 \cdot 10^{11}$ & 5 & 2142 \\
\hline LF-MMM & 182 & 778 & $1 \cdot 10^{11}$ & 6 & 1915 \\
\hline
\end{tabular}





\title{
Chapter 4
}

\section{Development of hollow fiber hemodialysis membranes for outside-in filtration}

\author{
I. Geremia ${ }^{\mathrm{a}}$, L. Bolhuis - Versteeg ${ }^{\mathrm{a}}, \mathrm{T} . \mathrm{Kelly}^{\mathrm{b}}, \mathrm{M} . \mathrm{Labib}^{\mathrm{c}}$ and D. Stamatialis ${ }^{\mathrm{a}}$ \\ a (Bio)artificial Organs, Department of Biomaterials Science and Technology, TechMed Centre, \\ Faculty of Science and Technology, University of Twente, P.O. Box 217, 7500 AE Enschede, The \\ Netherlands \\ ${ }^{\mathrm{b}}$ Medcatalyst Consulting LLC 118 Leonard Wood S \#202, Highland Park, IL, 60035, USA \\ ${ }^{\mathrm{c}}$ Novaflux, Inc., 1 Wall St., Princeton, NJ, 08540, USA
}




\section{Abstract}

In standard hemodialysis (HD), the blood flows in the lumen of hollow fiber hemodialyzer, while the dialysate into the inter-fiber space (IFS), in the so-called inside-out filtration mode. There thrombus can be deposited and blood clots can be formed at the inlet to the fiber leading to blocking of blood flow through the fiber and consequent to lowering of blood clearance and of filter life. The outsidein filtration (OIF), where the blood flows into the IFS and the dialysate flows in the fiber lumen could be applied to HD to prolong the operational hemodialyzer life. However, to achieve this one needs to develop new HD membranes suitable for OIF having outer blood compatible selective surface. Therefore, in this work, we investigate the development of hollow fibers suitable for OIF HD based on polyethersulfone/polyvinylpyrrolidone polymer blends. To achieve this, we tailor various spinning parameters, including dope and bore solutions composition, dope and bore flow rates. The resulting membranes have outer selective layer, high ultrafiltration coefficient $\left(>15 \mathrm{~mL} /\left(\mathrm{m}^{2} \cdot \mathrm{h} \cdot \mathrm{mmHg}\right)\right)$, low albumin leakage, very good creatinine clearance from human plasma as well as very good mechanical properties, matching successfully the properties of HD membranes used currently in the inside-out filtration mode. 


\section{Introduction}

End-stage kidney disease (ESKD) patients waiting for or not suitable for kidney transplantation need to undergo hemodialysis (HD) therapy. During HD, a semi-permeable membrane is responsible for the transfer of accumulated uremic solutes from the blood compartment to the dialysate compartment. Usually, HD treatment is performed three times per week and each session lasts four hours. Nevertheless, several studies have shown that prolonged HD sessions, for example performing extended nocturnal dialysis, would improve the removal of toxins from the blood of ESKD patients and may thereby increase patients' overall health and quality of life [1-6].

However, current commercial dialyzers are not designed to operate for long-term HD treatments. In standard HD, the blood flows in the intra-lumen space of the hollow fiber (HF) membranes, while the dialysate fluid into the inter-fibers space (IFS). In this mode of application, the so called "inside-out mode", thrombosis derived fiber clogging could limit the use of the dialyzers for prolonged time [7, 8]. Thrombus is deposited and blood clots are formed with contact to the artificial surfaces of a hemodialyzer. Clots form at the inlet to the fiber lumen, so the entire fiber is blocked, effectively stopping the blood flow through the individual fiber. As more fibers are blocked there is a decrease of the available membrane surface for blood detoxification and an increase in pressure [7]. When many fibers in the filter become clotted, the filter has to be replaced. Despite significant advances on improving membrane hemocompatibility over the last decades, the maximum fiber life is typically in the range of 15-40 hours even with the use of complex citrate anti-coagulation regimes $[7,9,10]$. In order to prevent fiber clotting, in 1992 Catapano et al. discussed the concept of "outside-in filtration" mode (OIF) for blood oxygenators [11]. In the OIF mode, the blood flows in the IFS instead of through the fiber lumen and it was found that thrombi deposited in the IFS have minimal impact on membrane function thanks to the fact that there the blood can flow around and bypass them. Hence, the effective membrane surface area is not reduced and mass transport disturbance is lower compared to the standard inside-out mode $[11,12]$. 
Only recently, the concept of OIF mode has been applied to hemodialysis. Dukhin et al. [7] showed that commercial dialyzers could operate for more than 100 hours when applied in the OIF mode. When the dialyzer operates in OIF mode, even though thrombosis will unavoidably occur, the thrombi will deposit in the IFS at the blood entrance. However, in OIF these small inlet thrombi have minimal effect on the blood flow and filtrate flux due to the three-dimensional system of interconnected hydrodynamic flow channels in the inter fiber space (IFS). Blood continues to flow through the filter and there is no appreciable loss of surface area (only 1-2\%), leading to only a minimal disturbance of the blood detoxification compared to standard HD [7]. Besides higher toxin removal, the longer operational time obtained with the OIF mode may lead to other several advantages, including a reduction of filter replacement costs, lower anti-coagulation requirements, lower blood loss, significant reduction of nursing time, lower disturbances in patient blood pressure and reduction of the likelihood of infection [7]. Importantly, the prolonged operational time of dialyzers and the higher membrane surface area (in comparison to the inside-out filtration) achieved during OIF could favor miniaturization and the clinical implementation of portable (PAK) and/or wearable artificial kidney (WAK) devices.

The HF membranes for OIF HD must have a smooth blood compatible thin selective layer on the outside fiber surface, for better hemocompatibility, lower protein adsorption and lower blood cells entrapment. Moreover, the membranes should have high ultrafiltration coefficient (KUf), in the range 8-60 $\mathrm{mL} /\left(\mathrm{m}^{2} \cdot \mathrm{h} \cdot \mathrm{mmHg}\right)$ with no appreciable albumin loss whether through sieving or membrane adsorption. Finally, the HF dimensions should be small to allow the assembling of several thousands of fibers into the dialyzers. To the best of our knowledge, there are only few studies on the development of HF for OIF and those have important limitations. Krause and co-inventors [13] produced HF based on polysulfone, polyethersulphone (PES) or polyarylethersulphone and a blend of at least two homo-polymers of polyvinylpyrrolidone (PVP), one low molecular weight (MW) PVP $(30-60 \mathrm{kDa})$ and one high MW PVP $(800-2000 \mathrm{kDa})$ with KUf in the range $4.7-470$ $\mathrm{mL} /\left(\mathrm{m}^{2} \cdot \mathrm{h} \cdot \mathrm{mmHg}\right)$. The described membranes have high molecular weight cut-off (MWCO), up to 
100000 Dalton, thus are not suitable for HD applications. Moreover, the manufacturing protocol is complex, including the heating of the spinneret and of the coagulation bath, which is a mixture of solvent and water. Gorsuch and co-inventors [14] also reported the development of HF for OIF mode composed by PES blended with polyethylene oxide or polyethylene glycol or by polysulfone modified with polyethylene oxide - polyethylene glycol copolymer. However, these membranes had very high KUf $\left(180-2400 \mathrm{~mL} /\left(\mathrm{m}^{2} \cdot \mathrm{h} \cdot \mathrm{mmHg}\right)\right)$ and very high plasma flux $(3000-72000$ $\left.\mathrm{mL} /\left(\mathrm{m}^{2} \cdot \mathrm{h} \cdot \mathrm{mmHg}\right)\right)$, thus being suitable for plasmapheresis and not for HD. Xu et al. [15] and Qin et al. [16] also reported protocols for the production of PES based HF for OIF able to retain albumin and with KUf in the range $61-217 \mathrm{~mL} /\left(\mathrm{m}^{2} \cdot \mathrm{h} \cdot \mathrm{mmHg}\right)$ and $81-394 \mathrm{~mL} /\left(\mathrm{m}^{2} \cdot \mathrm{h} \cdot \mathrm{mmHg}\right)$, respectively, but the reported fibers are very large to be bundled in hemodialyzers.

In this work we investigate the development of HF for OIF mode based on PES and PVP, which are the most commonly used polymers for HD membranes production. PES is widely known for its thermal stability, mechanical strength, chemical inertness and resistance to all sterilization techniques [17]. PVP is a polar, physiologically inert water-soluble polymer used as hydrophilic agent. Thus, PVP, thanks to the formation of a hydration layer, can prevent adsorption and deposition of plasma proteins on the membrane surface [17]. We optimize the spinning parameters by tuning the polymer dope concentration, dope and bore flow rates, external shower, bore solution composition, air gap length and take-up wheel speed to produce thin OIF fiber suitable for application in HD. The produced OIF fibers are characterized in terms of morphology (using scanning electron microscopy), surface chemistry (using fourier transformed infrared spectroscopy and X-ray photoelectron spectroscopy), mechanical properties and transport characteristics (using pure water, albumin and vitamin B12 aqueous solutions and human blood plasma spiked with creatinine). Fresenius F8HPS commercial HF is used as control for comparison. 


\section{Materials and methods}

\subsection{Membrane fabrication}

The HF for OIF were produced via non-solvent induced phase separation. Table 1 summarizes the parameters tuned for the fabrication of 16 different fibers, indicated as OIF1, OIF2 etc. to OIF16. The polymer dope solutions were prepared by dissolving Ultrason E6020 PES (BASF, Ludwigshafen, Germany) and PVP K90 (MW $\approx 360 \mathrm{kDa}$, Sigma-Aldrich Chemie GmbH, Munchen, Germany) in ultrapure N-methyl pyrrolidone (NMP) (Acros Organics, Geel, Belgium). All dope polymer solutions (Table 1) were mixed on a roller bench for 3 days, then they were filtered using a Bekipor ST AL3 $15 \mu \mathrm{m}$ filter (Bekaert, Kortrijk, Belgium), transferred in stainless-steel syringes and left to degas for at least 24 hours. Afterwards, the syringe containing the dope solution was connected to a highpressure syringe pump and to a spinneret for the preparation of the fibers (Figure 1). Ultrapure water was used as shower solution (Fig. 1), which was pumped through the spinneret at $0.3 \mathrm{~mL} / \mathrm{min}$. With the term "shower" is meant the external coagulant solution pressurized through the outer orifice of the spinneret (Fig. 1). Mixtures of ultrapure water and NMP at different concentrations were used as bore solutions (Table 1). The air gap between the spinneret and the coagulation bath was adjusted to $0.6 \mathrm{~cm}$ or $1.2 \mathrm{~cm}$ depending on the produced fiber (Table 1). A take-up wheel was used for the collection of the produced fibers. Different flow rates of the dope and bore solutions and different speeds of the take-up wheel were used for the fiber fabrication, see Table 1. The coagulation bath consisted of demineralized water at room temperature, approximately $20{ }^{\circ} \mathrm{C}$. The fabricated membranes were washed several times with demineralized water to remove any remaining solvent and were stored for further use. Commercial fiber F8HPS (Fresenius) was used as reference fiber for comparison of the results (scanning electron microscopy (SEM) images of Fresenius F8HPS fiber are reported in Supplementary Information). 
Table 1. Spinning parameters used for the production of the OIF fibers. Main variables are highlighted. Shower flow rate is for all the OIF fibers $0.3 \mathrm{~mL} / \mathrm{min}$.

\begin{tabular}{|c|c|c|c|c|c|c|}
\hline $\begin{array}{l}\text { OIF } \\
\text { fiber }\end{array}$ & $\begin{array}{c}\text { Dope } \\
\text { (PES/PVP/NMP) } \\
(\text { wt } \%)\end{array}$ & $\begin{array}{c}\text { Bore liquid } \\
\text { (NMP/water) } \\
\text { (wt\%) }\end{array}$ & $\begin{array}{c}\text { Dope flow rate } \\
\text { (mL/min) }\end{array}$ & $\begin{array}{l}\text { Bore flow rate } \\
(\mathrm{mL} / \mathrm{min})\end{array}$ & $\begin{array}{c}\text { Air gap } \\
(\mathrm{cm})\end{array}$ & $\begin{array}{l}\text { Take-up wheel } \\
(\mathrm{m} / \mathrm{min})\end{array}$ \\
\hline OIF1 & \multirow{13}{*}{$15 / 7 / 78$} & $50 / 50$ & 1.00 & 0.9 & 0.6 & $9 \pm 1$ \\
\hline OIF2 & & $50 / 50$ & 1.00 & 0.4 & 0.6 & $9 \pm 1$ \\
\hline OIF3 & & $75 / 25$ & 1.00 & 0.4 & 0.6 & $9 \pm 1$ \\
\hline OIF4 & & $90 / 10$ & 1.00 & 0.4 & 0.6 & $9 \pm 1$ \\
\hline OIF5 & & $75 / 25$ & 0.60 & 0.4 & 0.6 & $9 \pm 1$ \\
\hline OIF6 & & $75 / 25$ & 0.40 & 0.4 & 0.6 & $9 \pm 1$ \\
\hline OIF7 & & $75 / 25$ & 0.40 & 0.2 & 0.6 & $9 \pm 1$ \\
\hline OIF8 & & $75 / 25$ & 0.40 & 0.1 & 0.6 & $9 \pm 1$ \\
\hline OIF9 & & $75 / 25$ & 0.35 & 0.1 & 0.6 & $9 \pm 1$ \\
\hline OIF10 & & $75 / 25$ & 0.30 & 0.1 & 0.6 & $9 \pm 1$ \\
\hline OIF11 & & $75 / 25$ & 0.25 & 0.1 & 0.6 & $9 \pm 1$ \\
\hline OIF12 & & $75 / 25$ & 0.20 & 0.2 & 0.6 & $14 \pm 1$ \\
\hline OIF13 & & $75 / 25$ & 0.20 & 0.2 & 0.6 & $18 \pm 1$ \\
\hline OIF14 & \multirow{3}{*}{$12 / 5.6 / 82.4$} & $75 / 25$ & 0.40 & 0.1 & 0.6 & $9 \pm 1$ \\
\hline OIF15 & & $75 / 25$ & 0.40 & 0.2 & 0.6 & $9 \pm 1$ \\
\hline OIF16 & & $75 / 25$ & 0.50 & 0.2 & 1.2 & $9 \pm 1$ \\
\hline
\end{tabular}

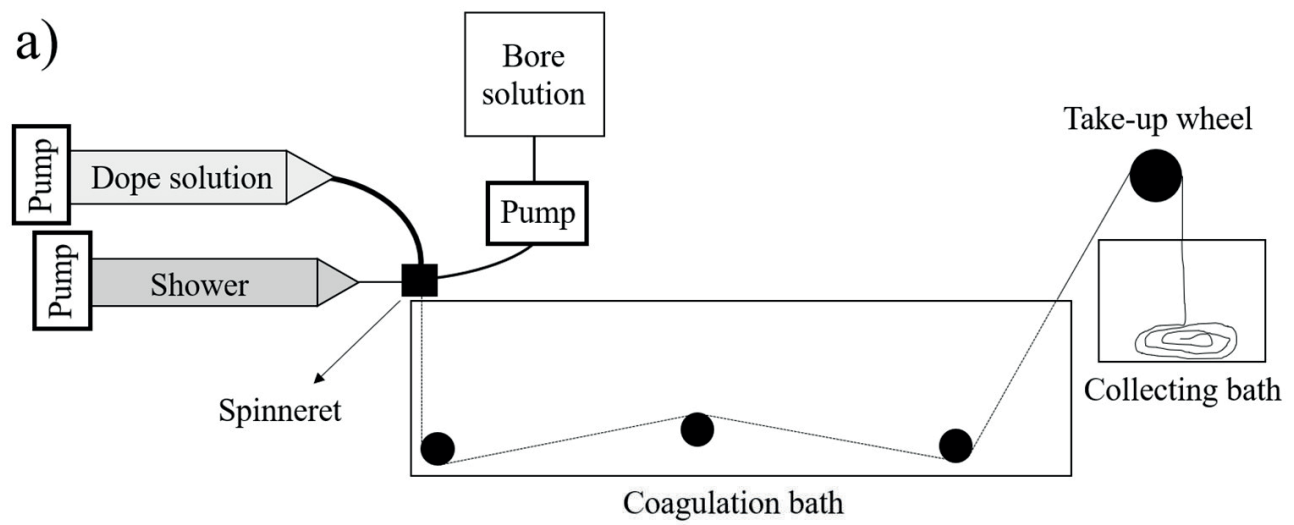

b)

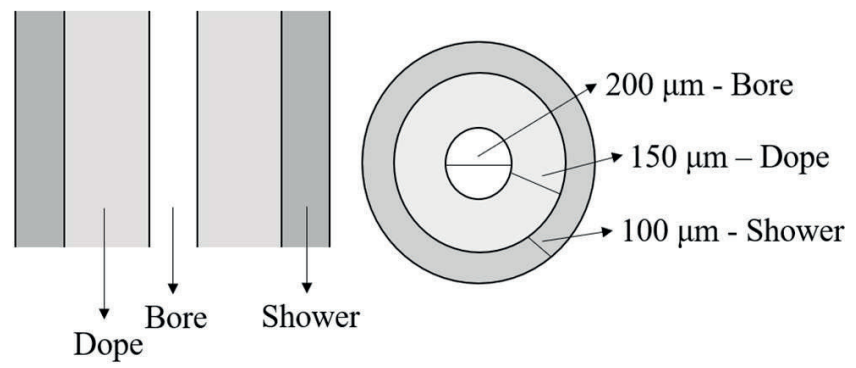

Figure 1. a) Scheme of the spinning set-up. b) Representation of the solutions at the exit of the spinneret (left) and specification of the spinneret dimensions (right). 


\subsection{Membrane characterization}

\subsubsection{Scanning electron microscopy (SEM)}

The morphology of the developed fibers was analyzed by SEM (JEOL JSM-IT 100, Tokyo, Japan). For the imaging of the cross-sections, the membranes were dried in air and fractured in liquid nitrogen. Prior to SEM imaging, the samples were gold sputtered using the Cressington 108 auto sputter (Cressington Scientific Instruments, Watford, UK).

\subsubsection{Attenuated Reflectance - Fourier Transmittance Infrared (ATR-FTIR)}

Analysis of the surface chemistry of selected fibers was performed by ATR-FTIR spectroscopy (Spectrum Two, PerkinElmer) and Spectrum Quant software. All scans were performed at room temperature in triplicate on various parts of the membrane surface at a resolution of $4 \mathrm{~cm}^{-1}$ and were compared to FTIR scans of pure PES, pure PVP materials and to commercial fiber Fresenius F8HPS.

\subsubsection{X-ray Photoelectron Spectroscopy (XPS)}

XPS analysis of selected fibers was performed using Quantera scanning XPS microprobe (Physical Electronics, Chanhassen, MN, USA) with Al Ka excitation radiation ( $h v=1486.6 \mathrm{eV})$. The given elemental atomic percentages were compared to Fresenius commercial fiber F8HPS. Data analysis was performed using Compass for XPS control, Multipak v 9.4.0.7.

\subsection{Transport experiments}

\subsubsection{Water transport}

Based on their morphology and size, OIF3, OIF4, OIF8, OIF14, OIF15 and OIF16 were selected for water transport measurements. They were dried in air and membrane modules with known surface areas (OIF3, $\mathrm{n}=3,4.1 \pm 0.8 \mathrm{~cm}^{2} ;$ OIF4, $\mathrm{n}=2,4.1 \pm 0.0 \mathrm{~cm}^{2} ;$ OIF8, $\mathrm{n}=2,2.6 \pm 0.0 \mathrm{~cm}^{2} ;$ OIF $14, \mathrm{n}$ 
$=2,2.7 \pm 0.0 \mathrm{~cm}^{2} ;$ OIF15, $\mathrm{n}=5,2.8 \pm 0.1 \mathrm{~cm}^{2} ;$ OIF $16, \mathrm{n}=5,4.8 \pm 2.9 \mathrm{~cm}^{2}$ ) were prepared using a 2-compontent epoxy glue (Griffon Combi Snel-Rapide, Bison International, Goes, The Netherlands). Before the water transport experiments, the fiber modules were pre-wetted with ethanol for 30 minutes at a transmembrane pressure (TMP) of $750 \mathrm{mmHg}$ and pre-compacted with ultra-pure water at a TMP of $750 \mathrm{mmHg}$ for at least $30 \mathrm{~min}$. Afterwards, the amount of permeated water was measured over time at TMP of 450, 600 and $750 \mathrm{mmHg}$. The resulting $\mathrm{KUf}\left(\mathrm{mL} /\left(\mathrm{m}^{2} \cdot \mathrm{h} \cdot \mathrm{mmHg}\right)\right)$ was calculated as the slope of the linear fit of the flux versus the TMP (in $\mathrm{mmHg}$ ) normalized by the surface area in $\mathrm{m}^{2}$

\subsubsection{Albumin transport}

After pre-wetting with ethanol at $750 \mathrm{mmHg}$ for at least 30 minutes and pre-pressurization with water at $750 \mathrm{mmHg}$ for at least 30 minutes, albumin filtration experiments were performed using membrane modules (prepared using a 2-component epoxy glue) with known effective membrane surface area (OIF3, $\mathrm{n}=2,3.6 \pm 0.6 \mathrm{~m}^{2} ;$ OIF4, $\mathrm{n}=2,4.1 \pm 0.0 \mathrm{~m}^{2} ;$ OIF8, $\mathrm{n}=2,2.6 \pm 0.0 \mathrm{~m}^{2} ;$ OIF $15, \mathrm{n}=5,2.8 \pm$ $0.1 \mathrm{~m}^{2}$; OIF16, $\mathrm{n}=4,5.8 \pm 3.1 \mathrm{~m}^{2}$ ) in dead-end configuration. For these experiments, bovine serum albumin (BSA) (66.5 KDa) was used (Sigma-Aldrich Chemie GmbH, Munchen, Germany). BSA solution at a concentration of $0.6 \mathrm{~g} / \mathrm{L}$ in Phosphate Buffer Saline (PBS) at pH 7.4 was pressurized in OIF mode at a pressure of $750 \mathrm{mmHg}$. After 30 minutes the permeate was collected and albumin concentration was measured using UV spectrophotometer (NanoDrop Technologies, Wilmington, DE). The albumin sieving coefficient (SC) was calculated using Equation 1.

$$
S C=\frac{C_{p}}{C_{f}}
$$

Equation 1

$C_{p}=$ Concentration permeate, $C_{f}=$ Concentration feed 


\subsubsection{Vitamin B12 transport}

After pre-wetting with ethanol at $750 \mathrm{mmHg}$ for at least 30 minutes and pre-pressurization with water at $750 \mathrm{mmHg}$ for at least 30 minutes, Vitamin B12 filtration experiments were performed on membrane modules of OIF16 $\left(\mathrm{n}=3,9.9 \pm 0.3 \mathrm{~m}^{2}\right)$ in dead-end configuration. Vitamin B12 solution at a concentration of $0.1 \mathrm{~g} / \mathrm{L}$ in PBS ( $\mathrm{pH} 7.4$ ) was pressurized in OIF mode from the dialysate compartment to the lumen compartment of the fibers at a pressure of $750 \mathrm{mmHg}$. After 30 minutes the permeate was collected and Vitamin B12 concentration was measured using UV spectrophotometer (NanoDrop Technologies, Wilmington, DE). The sieving coefficient was calculated using Equation 1.

\subsubsection{Creatinine transport experiments}

The removal of creatinine from human plasma by the OIF16 was investigated in diffusion mode (TMP $=0$ ) and in counter-current configuration using a dedicated set up (Convergence, Enschede, The Netherlands). $50 \mathrm{~mL}$ of human plasma (obtained by healthy donors in compliance with local ethical guidelines - Sanquin, Amsterdam, The Netherlands) spiked with creatinine (Sigma-Aldrich Chemie $\mathrm{GmbH}$, Schnelldorf, Germany) $(0.1 \mathrm{~g} / \mathrm{L})$ was recirculated at a flow rate of $10 \mathrm{~mL} / \mathrm{min}$ in the space outside the fibers (IFS). $50 \mathrm{~mL}$ of dialysate model solution was recirculated at a flow rate of $1 \mathrm{~mL} / \mathrm{min}$ in the intraluminal space. To prepare the dialysate model solution, $2 \mathrm{mM} \mathrm{KCl,} 140 \mathrm{mM} \mathrm{NaCl}, 1.5$ $\mathrm{mM} \mathrm{CaCl}_{2}, 0.25 \mathrm{mM} \mathrm{MgCl}_{2}, 35 \mathrm{mM} \mathrm{NaHCO}_{3}$ (all from Sigma-Aldrich Chemie GmbH, Schnelldorf, Germany) and $5.5 \mathrm{mM}$ glucose (Life Technologies Europe BV, Bleiswijk, The Netherlands) were dissolved in MilliQ water. Membrane modules composed of 3 fibers with a total outer surface area of $2.9 \pm 0.1 \mathrm{~cm}^{2}$ were used. The experiments were performed in triplicate for 24 hours and samples were taken every hour for the first 4 hours and at 24 hours from the blood plasma and dialysate compartments for quantification of creatinine. Creatinine concentrations were analyzed by UV-VIS using reverse-phase high-performance liquid chromatography (RP-HPLC). Creatinine concentrations were analyzed both in plasma (after filtration through $30 \mathrm{kDa}$ filters, Amicon Ultracel-30 K, Merck 
Millipore Ltd) and in the dialysate. Creatinine diffusive removal by the fibers was calculated by the creatinine concentration found in the dialysate. All results were normalized to the outer surface areas of the fiber modules.

\subsection{Mechanical properties}

The mechanical properties of the OIF16 and of the commercial F8HPS (Fresenius) fiber was performed using a Zwick Z020 tensile tester equipped with a $500 \mathrm{~N}$ load cell at room temperature. 5 $\mathrm{cm}$ long fiber samples were clamped at both ends and pulled at constant elongation velocity of 50 $\mathrm{mm} / \mathrm{min}$ till break. Ultimate tensile strength, Young's modulus and elongation at ultimate strength were obtained. In total, 13 samples of the OIF16 and 5 samples of F8HPS fibers were tested.

\subsection{Statistics}

All the data are presented as mean \pm SD (standard deviation). Statistical analyses were performed using GraphPad Prism version 5.02 (GraphPad Prism Software, La Jolla, CA, USA). Statistical differences for the mechanical test were determined using unpaired students' t-test. Multiple comparisons between different groups were performed using one-way analysis of variance (ANOVA) with Bonferroni post-hoc test in order to determine statistical differences of the thicknesses of the selective layers of the developed fibers. 


\section{Results and discussion}

\subsection{Membrane morphology characterization - Scanning electron microscopy (SEM)}

In this work all fibers were spun using the wet-wet spinning process. A triple-orifice spinneret was used (Fig. 1), with the bore liquid composition determining the lumen surface structure, while the outside liquid ("shower") was used to tailor the morphology and properties of the outer surface of the fibers [18]. After the contact with the first coagulant, the shower, the fibers enter the coagulation bath (second coagulant). Water, a strong non-solvent for PES [15], was chosen as coagulant for both the shower liquid and the coagulation bath. A flow rate of $0.3 \mathrm{~mL} / \mathrm{min}$ was selected for the water shower because it allowed to obtain regular dense outside layer without morphological irregularities. When higher shower flow rates were used the morphology of the fibers was quite irregular, while when applying lower flow rates, it was not possible to obtain dense outer layer (results not shown).

HF to be used for HD in OIF mode must have the selective layer at the outer surface and be impermeable to albumin. To achieve this, we investigated in detail various spinning parameters including the bore solution composition, the dope and bore flow rates, the take-up wheel speed, the air-gap length and the polymer dope concentration. Figures 2-5 present typical SEM images and Table 2 summarizes the morphological characteristics of the fabricated fibers. In the next sections we will discuss in detail our results.

\subsubsection{Effect of NMP concentration in the bore solution}

In order to avoid the formation of the selective layer at the lumen side, we investigated the effect of the addition of solvent (NMP) in the bore solution. This is expected to delay the diffusion of NMP out of the nascent fiber leading to slower phase separation [18] and, therefore, to more open membrane structure at the lumen side $[16,18,19]$. Three different NMP concentrations $(50,75$ and $90 \mathrm{wt} \%$ ) in the bore fluid were used (Table 1). NMP concentration of $50 \mathrm{wt} \%$ leads to fibers (OIF1 and OIF2) with rather dense inner layer, see Figure 2. When the concentration of NMP is increased 
to $75 \mathrm{wt} \%$ (OIF 3) and $90 \mathrm{wt} \%$ (OIF 4), an open lumen layer is obtained, while the outer layer remains dense (Fig. 2). Moreover, the lumen layer of OIF4 presents much larger pores compared to OIF3. This could be due to the fact that when the solvent concentration in the bore solution is higher than in the nascent fiber, the solvent might even diffuse into the nascent fiber, diluting the polymer concentration prior to phase separation [18]. The OIF 1-4 present spongy structure, suggesting that with these spinning parameters and bore compositions used, the phase separation is slow enough to avoid the formation of macrovoids. The increase of NMP concentration in the bore solution from 50 $\mathrm{wt} \%$ to $75 \mathrm{wt} \%$ also leads to an increase of $30 \mu \mathrm{m}$ of the inner diameter and to a $30 \mu \mathrm{m}$ thicker membrane wall (Table 2). This increase might also be related to the slow phase separation and low shrinkage due to the presence of high solvent amount in the bore solution. The thicknesses of the selective layers of OIF2 $(2.6 \pm 0.6 \mu \mathrm{m})$ and OIF4 $(2.1 \pm 0.0 \mu \mathrm{m})$ are similar; however, the selective layer of OIF3 is much thicker $(6.9 \pm 0.2 \mu \mathrm{m}$, significant different per $\mathrm{p}<0.0001)$ (Fig. 2). 


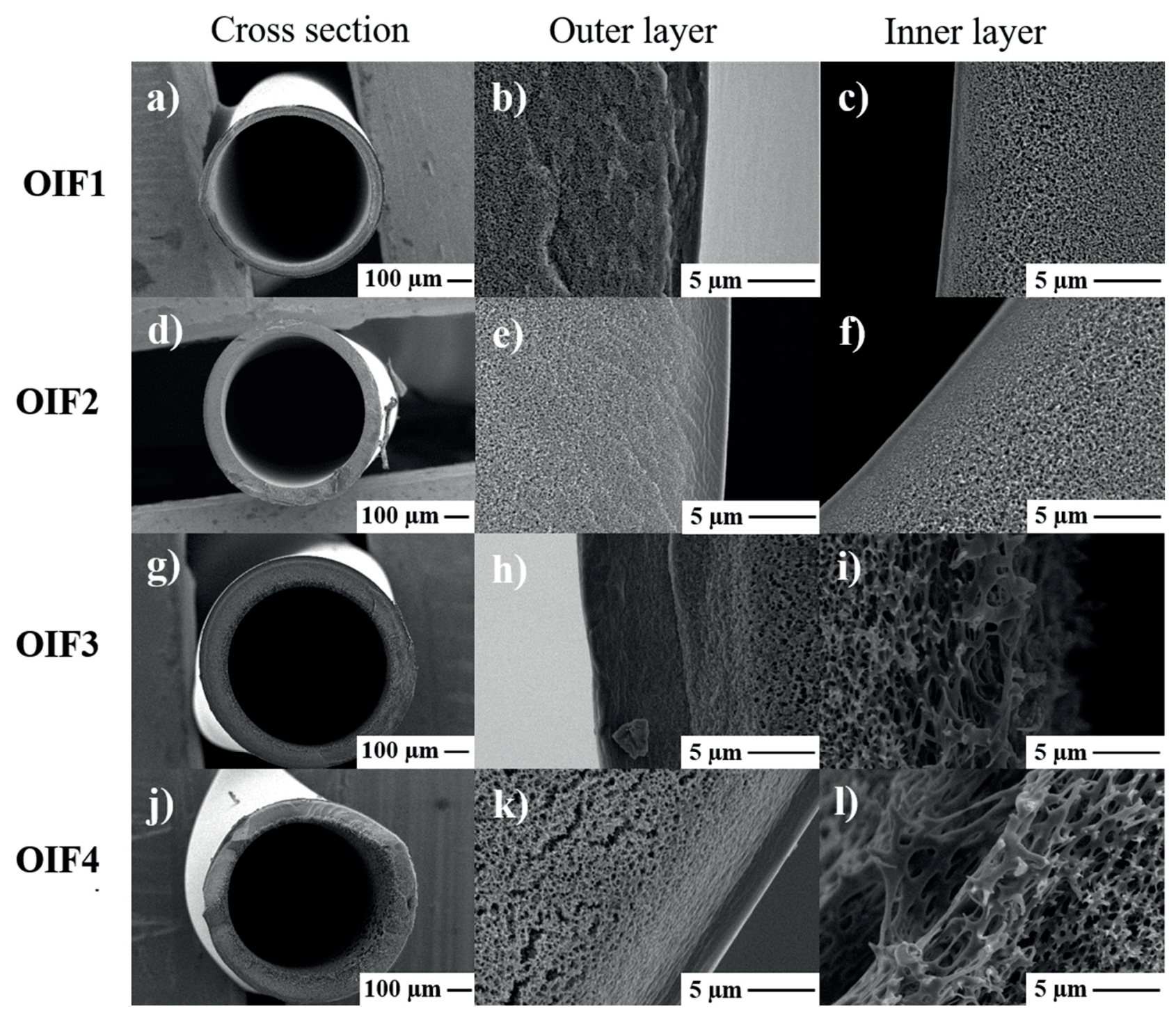

Figure 2. SEM images of OIF1, OIF2, OIF3 and OIF4. a, d, g and j) cross sections; b, e, h, k) magnifications of the outer layer; c, f, i, l) magnification of the inner layer. 


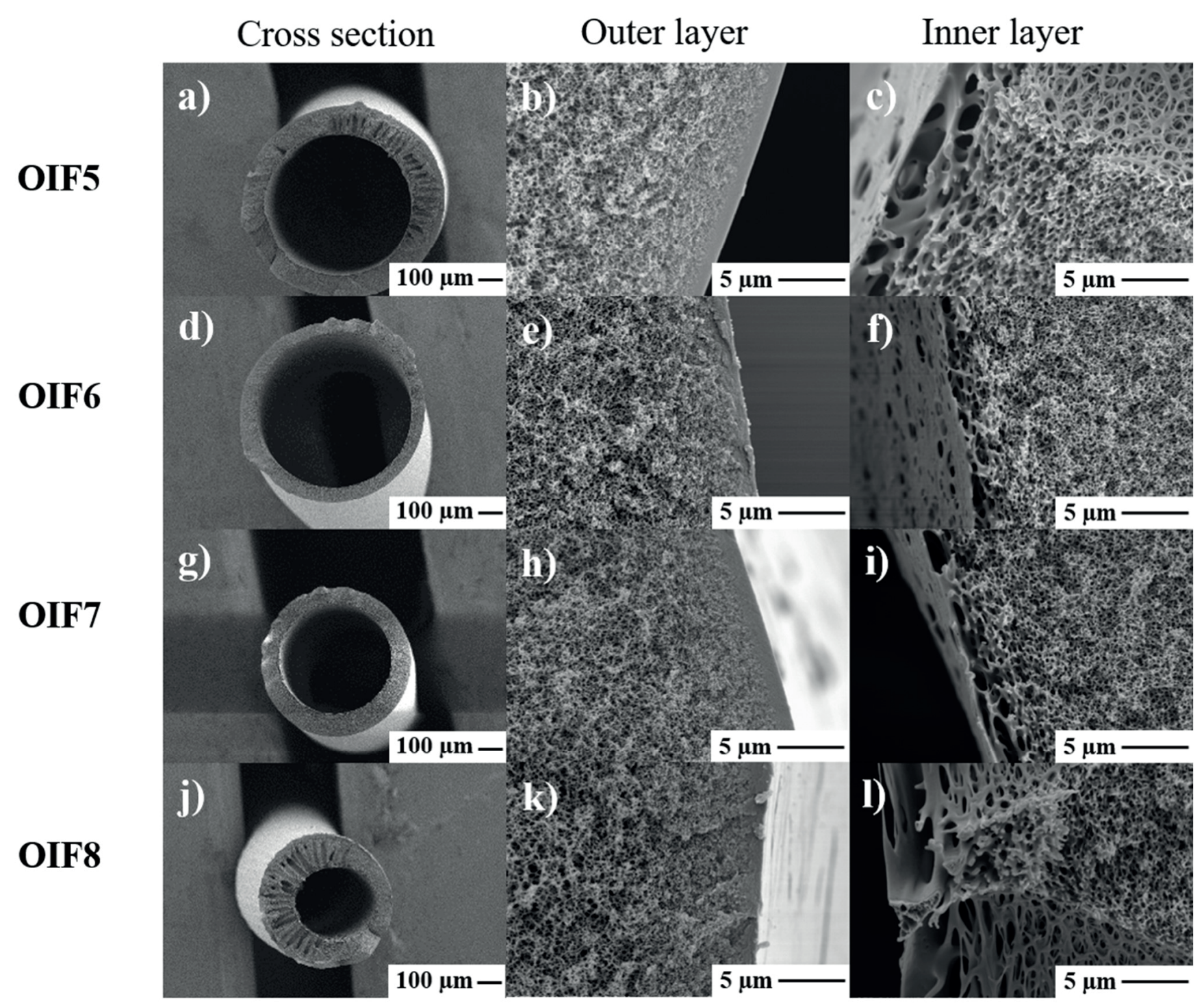

Figure 3. SEM images of OIF5, OIF6, OIF7 and OIF8. a, d, g and j) cross sections; $b, e, h, k$ ) magnifications of the outer layer; c, f, i, l) magnification of the inner layer.

\subsubsection{Effect of polymer dope flow rate}

In order to obtain small size fibers, which can be easily bundled in modules, the dope flow rate was tailored too. As expected $[16,20]$, by keeping all the other spinning parameters constant, the decrease of the dope flow rate (for OIF3: $1 \mathrm{~mL} / \mathrm{min}$, for OIF5: $0.6 \mathrm{~mL} / \mathrm{min}$ and for OIF6: $0.4 \mathrm{~mL} / \mathrm{min}$ ) leads to a significant reduction of the membrane wall thickness (approximately $70 \mu \mathrm{m}$ for OIF3, $65 \mu \mathrm{m}$ for OIF5 and $34 \mu \mathrm{m}$ for OIF6) (see Fig. 2, 3 and Table 2). Also, the thickness of the selective layer decreases with the reduction of the dope flow rate (OIF 3: $6.9 \pm 0.2 \mu \mathrm{m}$, OIF 5: $1.4 \pm 0.0 \mu \mathrm{m}$ and OIF $6: 1.3 \pm 0.4 \mu \mathrm{m})$ 
In order to produce even smaller fibers, the dope flow rate was further reduced for the fibers OIF8 OIF11 (0.4 mL/min, $0.35 \mathrm{~mL} / \mathrm{min}, 0.3 \mathrm{~mL} / \mathrm{min}$ and $0.25 \mathrm{~mL} / \mathrm{min}$, respectively) at a fixed bore flow rate of $0.1 \mathrm{~mL} / \mathrm{min}$. However, even though a significant decrease of the membrane size actually occurs (both wall thickness and inner diameter are reduced), the morphology of OIF9, OIF10 and OIF11 is irregular, with delamination of the inner lumen layer and not circular inner and outer circumference (see Fig. 4, Table 2). Based on these results, we concluded that when using very low bore flow rate of $0.1 \mathrm{~mL} / \mathrm{min}$, the lowest dope flow rate which can be used to produce fibers with regular shape is $0.4 \mathrm{~mL} / \mathrm{min}$. Moreover, it is interesting to observe that finger-like macrovoids are mainly found for the fibers OIF9-16, while the fibers OIF1-8 have, mainly, a spongy porous morphology. OIF 9-16 were spun using lower dope and bore flow rates compared to the other fibers, but with the same shower flow rate $(0.3 \mathrm{~mL} / \mathrm{min})$. It seems that due to the faster diffusion of the external coagulant (water shower) into the forming fiber compared to the outflow of solvent (NMP) for OIF 9-16, instantaneous demixing takes place, leading to the formation of macrovoids, in agreement to other studies $[15,18,21]$. 


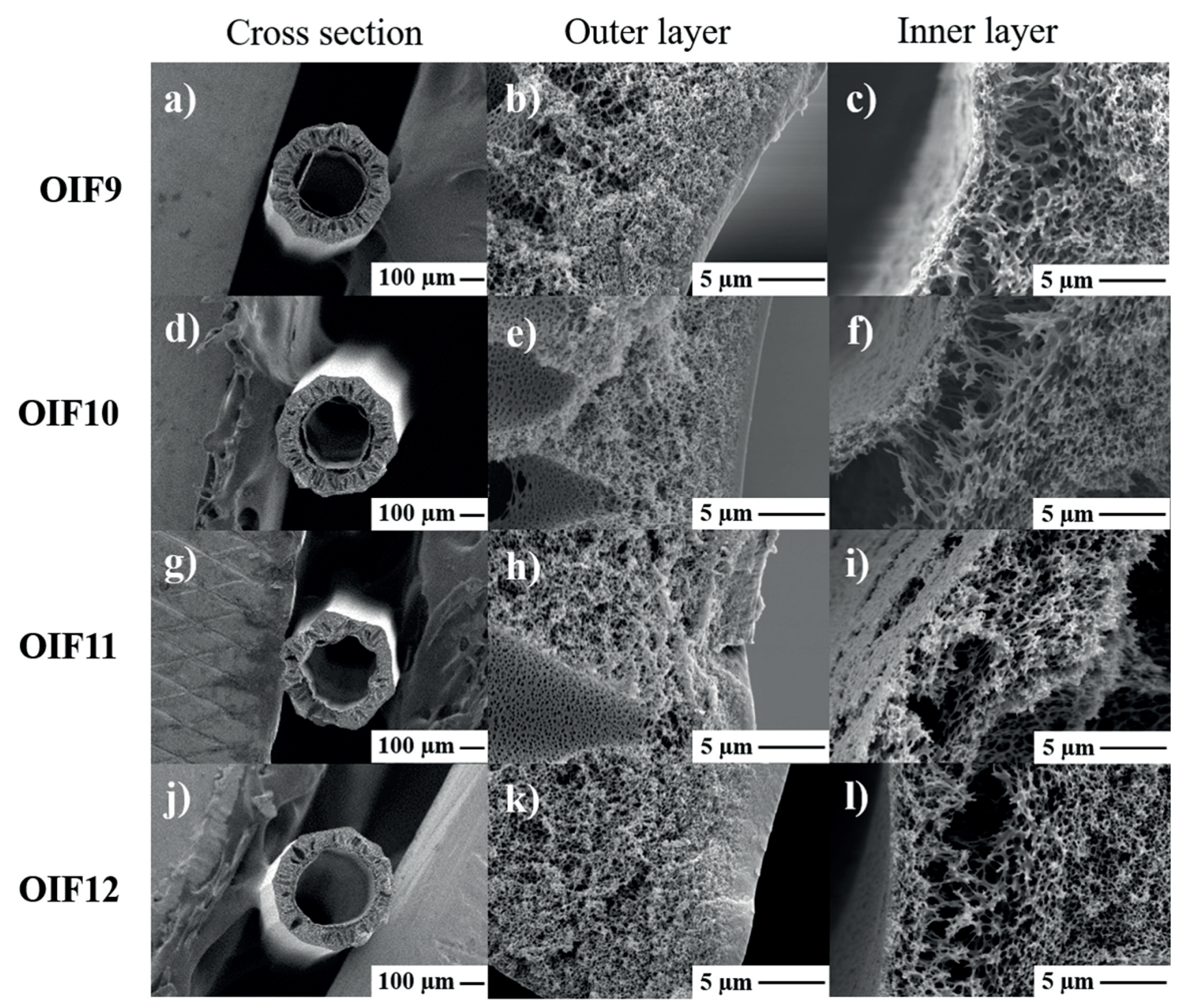

Figure 4. SEM images of OIF9, OIF10, OIF11 and OIF12. a, d, g, j) cross sections; b, e, h, k) magnifications of the outer layer; c, f, i, l) magnification of the inner layer.

\subsubsection{Effect of bore flow rate}

The effect of the bore flow rate on the membrane dimensions was also studied. It is expected that lower bore flow rates would lead to smaller inner fiber diameter [22]. Indeed, for the OIF1 and OIF2 the decrease of the bore flow rate from $0.9 \mathrm{~mL} / \mathrm{min}$ to $0.4 \mathrm{~mL} / \mathrm{min}$, respectively, results in a significant decrease of the inner diameter of about $200 \mu \mathrm{m}$ (Fig. 2, Table 2). When the bore flow rate is further reduced, for OIF $6(0.4 \mathrm{~mL} / \mathrm{min})$, OIF $7(0.2 \mathrm{~mL} / \mathrm{min})$ and OIF $8(0.1 \mathrm{~mL} / \mathrm{min})$ at a constant dope flow rate of $0.4 \mathrm{~mL} / \mathrm{min}$, the inner diameter of the fibers decreases considerably from about $369 \mu \mathrm{m}$ (OIF6) to $276 \mu \mathrm{m}$ (OIF7) and $191 \mu \mathrm{m}$ (OIF8); however, the wall thickness increases from $34 \mu \mathrm{m}$ 
(OIF6) to $72 \mu \mathrm{m}$ (OIF8) (Fig. 3, Table 2). In the case of OIF14 and OIF15, as a consequence of the increase of the bore flow rate from $0.1 \mathrm{~mL} / \mathrm{min}$ (OIF14) to $0.2 \mathrm{~mL} / \mathrm{min}$ (OIF 15), the inner diameter of the OIF15 increases by approximately $40 \mu \mathrm{m}$ (Fig. 5, Table 2). However, the morphology of the OIF14 is rather irregular along the fiber length but the OIF15 has regular and reproducible morphology all along the fiber length. Finally, the bore flow rate does not have any effect on the thickness of the selective layer: for the OIF1 and OIF2 is $2.4 \pm 0.3 \mu \mathrm{m}$ and $2.6 \pm 0.4 \mu \mathrm{m}$, respectively; for the OIF6, OIF7, OIF8 is $1.3 \pm 0.4 \mu \mathrm{m}, 1.3 \pm 0.0 \mu \mathrm{m}, 1.5 \pm 0.1 \mu \mathrm{m}$, respectively and for the OIF14 and OIF15 is $0.9 \pm 0.0 \mu \mathrm{m}$ and $0.7 \pm 0.1 \mu \mathrm{m}$, respectively.

\subsubsection{Effect of take-up wheel speed}

In order to reduce the fiber dimensions, the effect of the take-up wheel speed was also studied since it is expected that faster take-up speed would decrease the overall size of the fibers due to the pulling action [23]. In fact, all the fibers produced in this work were spun using a take-up wheel speed of $9 \pm$ $1 \mathrm{~m} / \mathrm{min}$, except for the OIF12 and OIF13 which were spun with speed of $14 \pm 1 \mathrm{~m} / \mathrm{min}$ and $18 \pm 1$ $\mathrm{m} / \mathrm{min}$, respectively. This leads as expected, to an overall decrease of the fiber's sizes but, similar to the findings for the OIF9-11, the OIF12 and OIF13 have irregular morphology characterized by delamination of the inner layer and irregular outer and inner circumferences (Fig. 4 and Table 2). 


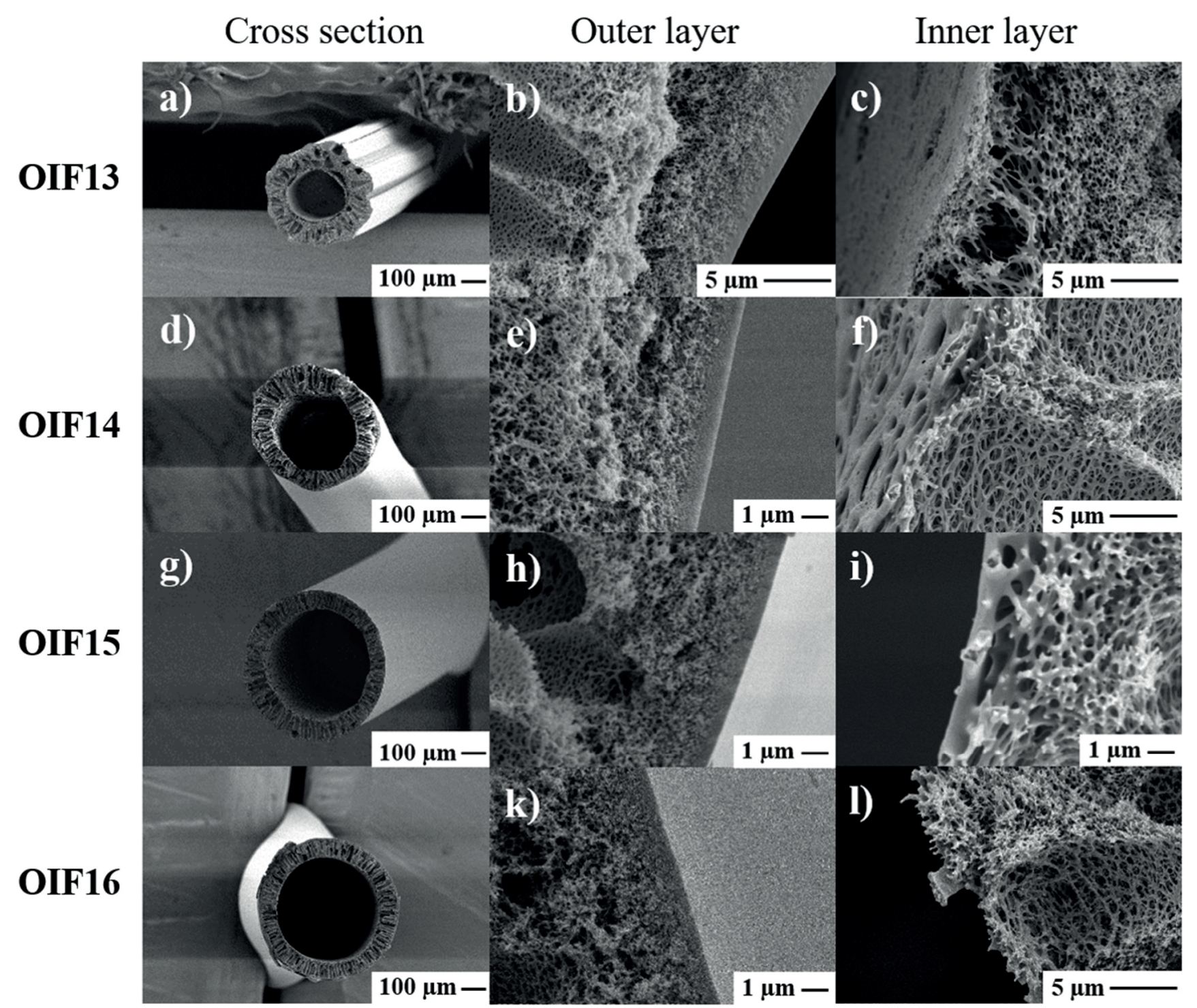

Figure 5. SEM images of OIF13, OIF14, OIF15 and OIF16. a, d, g, j) cross sections; b, e, h, k) magnifications of the outer layer; c, f, i, l) magnification of the inner layer. 
Table 2. Summary of the characteristics of the developed fibers. "OD" outer diameter, "ID" inner diameter. Fibers highlighted in grey were selected for further characterization.

\begin{tabular}{|c|c|c|c|c|c|}
\hline OIF fiber & $\begin{array}{l}\text { O.D. } \\
(\mu \mathrm{m})\end{array}$ & $\begin{array}{l}\text { I.D. } \\
(\mu \mathrm{m})\end{array}$ & $\begin{array}{l}\text { Wall thickness } \\
\qquad(\mu \mathrm{m})\end{array}$ & $\begin{array}{c}\text { Selective layer thickness }(\mu \mathrm{m}) \\
\text { and location }\end{array}$ & Comments \\
\hline OIF1 & 643 & 564 & 43 & $\begin{array}{c}2.4 \pm 0.3, \text { outside (dense layer } \\
\text { also in the inside) }\end{array}$ & No further characterized \\
\hline OIF2 & 448 & 368 & 41 & $\begin{array}{c}2.6 \pm 0.6, \text { outside (dense layer } \\
\text { also in the inside) }\end{array}$ & No further characterized \\
\hline OIF3 & 534 & 398 & 70 & $6.9 \pm 0.2$, outside & Further characterized \\
\hline OIF4 & 503 & 390 & 50 & $2.1 \pm 0.0$, outside & Further characterized \\
\hline OIF5 & 497 & 364 & 65 & $1.4 \pm 0.0$, outside & $\begin{array}{l}\text { Large fiber, no further } \\
\text { characterized }\end{array}$ \\
\hline OIF6 & 437 & 369 & 34 & $1.3 \pm 0.4$, outside & $\begin{array}{l}\text { Large fiber, no further } \\
\text { characterized }\end{array}$ \\
\hline OIF7 & 370 & 276 & 49 & $1.3 \pm 0.0$, outside & $\begin{array}{l}\text { Large fiber, no further } \\
\text { characterized }\end{array}$ \\
\hline OIF8 & 330 & 191 & 72 & $1.5 \pm 0.1$, outside & Further characterized \\
\hline OIF9 & 310 & 190 & 61 & Not measured, outside & $\begin{array}{c}\text { Irregular shape and inner layer } \\
\text { delamination. No further } \\
\text { characterized }\end{array}$ \\
\hline OIF10 & 294 & 188 & 47 & Not measured, outside & $\begin{array}{c}\text { Irregular shape and inner layer } \\
\text { delamination. No further } \\
\text { characterized }\end{array}$ \\
\hline OIF11 & 272 & 166 & 55 & Not measured, outside & $\begin{array}{c}\text { Irregular shape and inner layer } \\
\text { delamination. No further } \\
\text { characterized }\end{array}$ \\
\hline OIF12 & 297 & 196 & 50 & Not measured, outside & $\begin{array}{c}\text { Irregular shape and inner layer } \\
\text { delamination. No further } \\
\text { characterized }\end{array}$ \\
\hline OIF13 & 252 & 124 & 67 & Not measured, outside & $\begin{array}{c}\text { Irregular shape and inner layer } \\
\text { delamination. No further } \\
\text { characterized }\end{array}$ \\
\hline OIF14 & 317 & 201 & 52 & $0.9 \pm 0.0$, outside & $\begin{array}{c}\text { Irregular morphology, only } \\
\text { water transport measurement }\end{array}$ \\
\hline OIF15 & 338 & 243 & 48 & $0.7 \pm 0.1$, outside & Further characterized \\
\hline OIF16 & 322 & 236 & 42 & $0.6 \pm 0.1$, outside & Further characterized \\
\hline
\end{tabular}




\subsubsection{Effect of polymer concentration}

The OIF14, OIF15 and OIF16 were spun using lower polymer concentration (PES $12 \mathrm{wt} \%$, PVP 5.6 $w t \%$, NMP $82.4 \mathrm{wt} \%$ ) compared to all the other fibers (PES $15 \mathrm{wt} \%$, PVP $7 \mathrm{wt} \%$, NMP $78 \mathrm{wt} \%$ ). Application of lower polymer concentrations is expected to produce fibers with overall smaller size (especially thinner walls) and higher KUf [24]. In fact, the morphologies of OIF14 and OIF15 can be compared to those of OIF8 and OIF7, respectively. OIF14 was, indeed, spun using the same spinning parameters as for the spinning of OIF8 and the spinning of OIF15 with the same spinning parameters as OIF7, with the only exception of lower polymer dope concentration (Table 1). Even though both OIF14 and OIF8 have rather non uniform structure, still one can see that the average wall thickness of OIF14 is approximately $20 \mu \mathrm{m}$ thinner compared to that of OIF8. However, the lower polymer concentration applied for the fabrication of OIF15 leads to a reduction of the inner diameter of about $30 \mu \mathrm{m}$, instead to a decrease of the membrane wall thickness. Moreover, the decrease of the polymer dope concentration seems to reduce the thickness of the selective layer, too. In fact, the thickness of the selective layer of OIF14 $(0.9 \pm 0.1 \mu \mathrm{m})$ and $\operatorname{OIF} 15(0.7 \pm 0.1 \mu \mathrm{m})$ are significantly lower compared to those of OIF8 $(1.5 \pm 0.1 \mu \mathrm{m})$ and $\operatorname{OIF} 7(1.3 \pm 0.0 \mu \mathrm{m})$.

\subsubsection{Effect of air gap}

Unlike all other fibers for which an air gap of $0.6 \mathrm{~cm}$ was used, OIF16 (Fig. 5) was produced with an air gap of $1.2 \mathrm{~cm}$. Direct comparison of OIF16 with other fibers cannot be made since more parameters than the air gap were tailored. Some comparison can be made with OIF15, for which, besides the shorter air gap, a lower dope flow rate $(0.4 \mathrm{~mL} / \mathrm{min}$ instead of $0.5 \mathrm{~mL} / \mathrm{min})$ was used. Despite the faster dope flow rate, which is expected to increase the size of the fibers (especially the wall thickness), the dimensions of OIF16 are slightly smaller compared to OIF15 (Fig. 5, Table 2). This can be attributed to the longer air gap, which introduces an elongational stress on the fiber because of gravity $[15,18,23,25]$. In fact, polymer macromolecules may undergo die-swell when they exit the spinneret, but when the air gap is large, the swell may disappear before the nascent fiber 
enters the coagulation bath. With shorter air gap, the nascent fiber has no time to encounter elongation before entering the coagulation bath and the die-swelled fiber may be directly coagulated in water $[15,16]$

\subsection{Transport properties of the developed fibers for OIF}

\subsubsection{Water transport}

Based on their morphology and dimensions, OIF3, OIF4, OIF8, OIF14, OIF15 and OIF16 were selected for water transport measurements (see results in Table 3). The increase of NMP concentration in the bore solution from $75 \%$ to $90 \%$ leads to fibers with higher $\mathrm{KUf}\left(9.8 \pm 0.1 \mathrm{~mL} /\left(\mathrm{m}^{2} \cdot \mathrm{h} \cdot \mathrm{mmHg}\right)\right.$ for OIF3 and $238 \pm 45 \mathrm{~mL} /\left(\mathrm{m}^{2} \cdot \mathrm{h} \cdot \mathrm{mmHg}\right)$ for OIF4). As discussed earlier, the addition of solvent NMP in the bore solution slows down the phase separation thus contributing to the formation of more open fibers. The large difference between the water transport properties of OIF3 and OIF4 is also due to differences in their selective layer thickness $(6.9 \pm 0.2 \mu \mathrm{m}$ for OIF3 compared to $2.1 \pm 0.0 \mu \mathrm{m}$ for OIF4; Fig. 2, section 3.1.1). Despite the lower dope and bore flow rates used for the production of OIF8, this fiber presents comparable KUf $\left(11.3 \pm 1.3 \mathrm{~mL} /\left(\mathrm{m}^{2} \cdot \mathrm{h} \cdot \mathrm{mmHg}\right)\right)$ to that of OIF3. The thickness of the selective layers of OIF3 $(6.9 \pm 0.2 \mu \mathrm{m})$ and OIF8 $(1.5 \pm 0.1 \mu \mathrm{m})$ are quite different, however the porosity and sieving properties of the two fibers seem to be similar.

The effect of the polymer dope concentration on the membrane transport properties can be appreciated for OIF14 which was spun with the same spinning parameters as for OIF8, besides using lower polymer dope concentration (Table 1). Despite the thinner selective layer and thinner wall of the OIF8, the OIF14 has significantly higher KUf compared to OIF8. It is also important to note that the increase of the bore flow rate from $0.1 \mathrm{~mL} / \mathrm{min}$ (OIF14) to $0.2 \mathrm{~mL} / \mathrm{min}$ (OIF15) leads to approximately $50 \%$ reduction of the KUf. Perhaps the increase of the bore flow rate leads to faster phase separation and, consequently, to denser membrane structure. However, this is not yet totally clear especially because of the high concentration of solvent in the bore solution. Despite the faster 
dope flow rate and the longer air gap, OIF16 has similar KUf to that of OIF15. In fact, the morphology of the two fibers is quite similar (Fig. 5).

\subsubsection{Bovine serum albumin (BSA) filtration experiments}

Albumin filtration experiments were performed in the dead-end filtration mode by pressurizing BSA solution at a concentration of $0.6 \mathrm{~g} / \mathrm{L}$ in $\mathrm{PBS}(\mathrm{pH}$ 7.4) from the dialysate compartment to the lumen compartment of the fibers at a pressure of $750 \mathrm{mmHg}$ for the OIF3, OIF4, OIF8, OIF15, OIF16 and the calculated sieving coefficients (SC) (Equation 1) are shown in Table 3. Because of already mentioned structural defects of OIF14, we decided to not proceed further with its investigation.

The albumin SC of OIF3, OIF8 and OIF15 is low in accordance with their relatively low KUf values, while the OIF4 has high SC matching to its high KUf. Due to this, OIF4 was not characterized further in this study. OIF16 has slightly higher albumin SC compared to OIF3, OIF8 and OIF15, but still acceptable for HD applications. Based on the overall results related to fiber size, fiber morphology, transport properties and to the easier manufacturing of OIF16, due to the higher air gap applied compared to the other OIF fibers, OIF16 was selected for further studies.

Table 3. Transport characteristics of selected OIF. Values are shown as mean \pm standard deviation.

\begin{tabular}{|c|c|c|c|}
\hline Membrane & KUf $\left(\mathrm{mL} /\left(\mathrm{m}^{2} \cdot \mathbf{h} \cdot \mathbf{m m H g}\right)\right)$ & BSA, SC & Comments \\
\hline OIF3 & $9.8 \pm 0.1(\mathrm{n}=3)$ & $0.00 \pm 0.00(\mathrm{n}=2)$ & \multirow{6}{*}{ This study } \\
\hline OIF4 & $238 \pm 45(\mathrm{n}=2)$ & $0.98 \pm 0.00(\mathrm{n}=2)$ & \\
\hline OIF8 & $11.3 \pm 1.3(\mathrm{n}=2)$ & $0.02 \pm 0.01(\mathrm{n}=2)$ & \\
\hline OIF14 & $31.1 \pm 2.4(\mathrm{n}=2)$ & Not measured & \\
\hline OIF15 & $17 \pm 5.5(\mathrm{n}=5)$ & $0.02 \pm 0.01(\mathrm{n}=5)$ & \\
\hline OIF16 & $15 \pm 3.5(\mathrm{n}=5)$ & $0.05 \pm 0.04(\mathrm{n}=4)$ & \\
\hline F8HPS & $\begin{array}{c}10 \pm 4 \\
18\end{array}$ & $\begin{array}{l}0.03 \\
0.03\end{array}$ & $\begin{array}{l}{[26]} \\
{[27]}\end{array}$ \\
\hline
\end{tabular}




\subsubsection{Vitamin B12 filtration}

Vitamin B12 (MW $1355 \mathrm{Da}$ ), a neutral organic solute, is often used as a marker molecule for estimating the transport of middle size solutes across the HD membranes. Therefore, in this study, we also measured its transport across the OIF16 membrane by pressurizing Vitamin B12 solution at a concentration of $0.1 \mathrm{~g} / \mathrm{L}$ in PBS (pH 7.4) from the dialysate compartment to the lumen compartment of the fibers at a pressure of $750 \mathrm{mmHg}$. Its SC for the OIF16 was equal to $1.00(\mathrm{n}=3)$, as expected for HD membranes.

\subsubsection{Creatinine transport}

Figure 6 presents the kinetics of creatinine transport from human plasma across OIF16. The membrane removes approximately $2000 \mathrm{mg} / \mathrm{m}^{2}$ and $4700 \mathrm{mg} / \mathrm{m}^{2}$ in 4 and 24 hours, respectively.

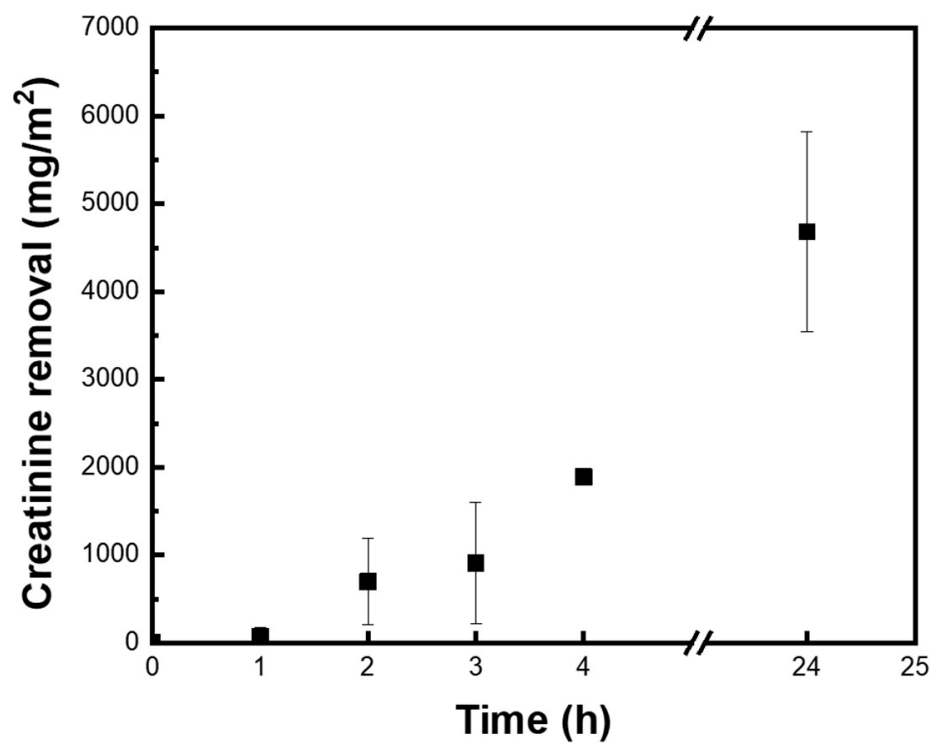

Figure 6. Creatinine removal by $\operatorname{OIF} 16(\mathrm{n}=3)$ from human plasma.

Based on the removal at $24 \mathrm{~h}$, considering a daily production of creatinine equal to $1800 \mathrm{mg}$ [28] and assuming the same creatinine removal in vivo, we estimate that $0.4 \mathrm{~m}^{2}$ of the OIF16 would be needed to remove the creatinine produced daily by an ESKD patient. This falls well into the range of dialyzers currently used in the clinic $\left(0.4-2.6 \mathrm{~m}^{2}\right)$ [29]. 
Direct comparison of creatinine removal by the OIF16 to that obtained by other HD membranes is quite difficult, since the later are actually operated in large modules, at higher flow rates of blood and dialysate and in the inside-out filtration mode. Nevertheless, the estimated creatinine clearance of OIF16, operated at plasma flow rate of $10 \mathrm{~mL} / \mathrm{min}$ in the IFS and dialysate flow rate of $1 \mathrm{~mL} / \mathrm{min}$ in the intraluminal space, is $90 \pm 28 \mathrm{~mL} /\left(\mathrm{min} \cdot \mathrm{m}^{2}\right)$ and is in the same range of the creatinine clearance reported for various HD membranes of comparable KUf, operated at higher blood and dialysate flow rates. For example, the creatinine clearance of F8HPS (KUf $=10\left(\mathrm{~mL} /\left(\mathrm{m}^{2} \cdot \mathrm{h} \cdot \mathrm{mmHg}\right)\right.$ ) is 124 $\mathrm{mL} /\left(\mathrm{m}^{2} \cdot \mathrm{min}\right)$ at blood flow rate $\mathrm{Qb}=300 \mathrm{~mL} / \mathrm{min}[30]$, the creatinine clearance of F7HPS $(\mathrm{KUf}=$ $10\left(\mathrm{~mL} /\left(\mathrm{m}^{2} \cdot \mathrm{h} \cdot \mathrm{mmHg}\right)\right)$ was $109 \mathrm{~mL} /\left(\mathrm{m}^{2} \cdot \mathrm{min}\right)$ at $\mathrm{Qb}=200 \mathrm{~mL} / \mathrm{min}[30]$ and the clearance of F10HPS $\left(\mathrm{KUf}=9.5\left(\mathrm{~mL} /\left(\mathrm{m}^{2} \cdot \mathrm{h} \cdot \mathrm{mmHg}\right)\right)\right.$ was $105 \mathrm{~mL} /\left(\mathrm{m}^{2} \cdot \mathrm{min}\right)$ at $\mathrm{Qb}=300 \mathrm{~mL} / \mathrm{min}[30]$. It is reasonable to assume that application of higher flow rates of plasma and dialysate in combination with the application of an alternative module design would further improve the clearance of the OIF16 membrane.

\subsection{Surface chemistry characterization}

In this study we used PES as membrane forming polymer. PES, on one hand, has exceptional filtering characteristics, thermal stability, mechanical strength, chemical inertness and it can withstand all sterilization techniques [31]. On the other hand, its hydrophobic nature favors proteins adhesion on the membrane, which not only affects membrane performance but also can trigger a series of other reactions such as activation of the coagulation cascade, blood clotting, complement and fibrinolysis reactions $[31,32]$. Blending of PES with hydrophilic additives leads to the reduction of protein adhesion and to improved blood compatibility [31-33]. In this work, PVP was used as hydrophilic additive to PES and it was important to access whether it is distributed well across the fiber and especially whether it is present at the blood contacting outer selective layer of the OIF16. This was investigated by means of ATR - FTIR and XPS. Figure 7a compares the ATR - FTIR spectra of the outer surfaces of OIF16 and F8HPS (Fresenius) fibers to the spectra of pure powder of PES and PVP. 
The peak at $1677 \mathrm{~cm}^{-1}$, corresponding to the carbonyl groups of PVP, has noticeably higher intensity for OIF16 in comparison to the F8HPS, indicating higher concentration of PVP at the outer surface of the OIF16. XPS measurements (Fig. 7b) performed on the outer and inner surface of OIF16 and F8HPS fibers confirm that the PVP is well distributed within the OIF16. The nitrogen percentage (exclusively part of PVP molecules) on the outer surface of OIF16 is similar to that on the inner surface of F8HPS, which is widely used for hemodialysis thanks to its good hemocompatibility profile.

a)

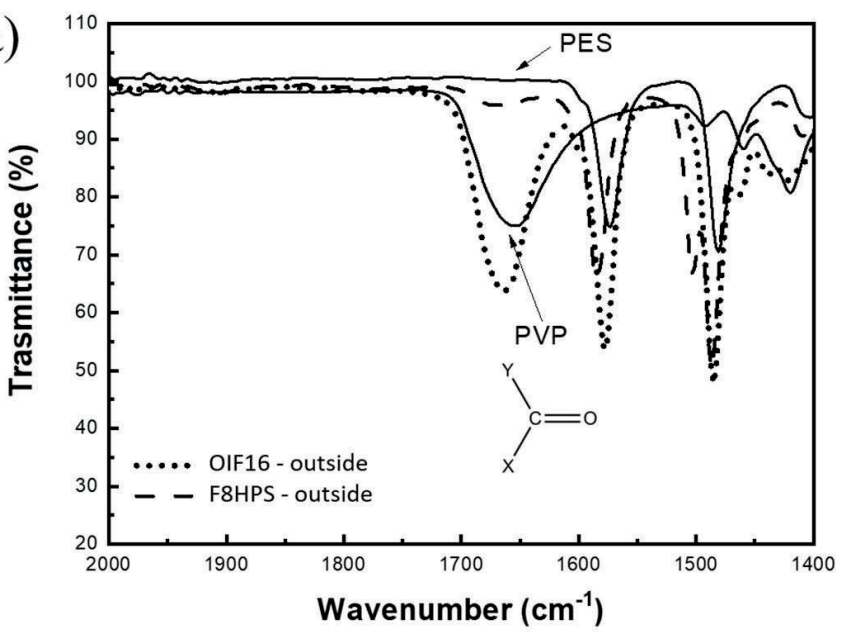

b)

\begin{tabular}{|l|c|c|c|c|}
\hline \multirow{2}{*}{} & \multicolumn{2}{|c|}{ OIF16 } & \multicolumn{2}{c|}{ F8HPS } \\
\cline { 2 - 5 } & Outside & Inside & Outside & Inside \\
\hline $\mathbf{C}(\%)$ & 75.79 & 75.30 & 80.43 & 82.08 \\
\hline $\mathbf{N}(\%)$ & $\mathbf{3 . 6 9}$ & 6.33 & 4.12 & $\mathbf{3 . 1 3}$ \\
\hline $\mathbf{O}(\%)$ & 17.26 & 15.18 & 13.67 & 12.67 \\
\hline Si (\%) & 3.26 & 3.19 & 1.78 & 2.12 \\
\hline $\mathbf{S ~ ( \% ) ~}$ & 1.1 & 2.0 & 2.3 & 1.5 \\
\hline
\end{tabular}

Figure 7. a) ATR-FTIR results of OIF16 and comparison to F8HPS fiber, pure PES and pure PVP materials. B) Elemental molar percentage results of OIF16 and F8HPS measured by XPS.

\subsection{Mechanical tests}

HD membranes should have enough mechanical strength to withstand mechanical stresses typical of HD treatment, especially when convection is applied, with little resistance for transport. Therefore, the mechanical properties of OIF16 were measured and compared to F8HPS fiber (Figure 8). On one hand, there is no significant difference between the two fibers concerning elastic deformation (Young's Modulus, Fig. 8a). On the other hand, the maximum strength and maximum elongation before break are lower for the OIF compared to the commercial fiber (Fig. 8b and 8c). Young's modulus is a measure of the stiffness of a material. Higher is the Young's modulus, smaller is the elastic strain of the material, indicating that the material is more rigid. Greater values of the Young's 
modulus help maintaining the integrity and the size of the membrane pores under pressure application. Therefore, the Young's modulus is the value particularly relevant for hemodialysis application. Based on this and thanks to the comparable Young's modulus to F8HPS fiber, we can conclude that the OIF16 can withstand typical stresses in hemodialysis.
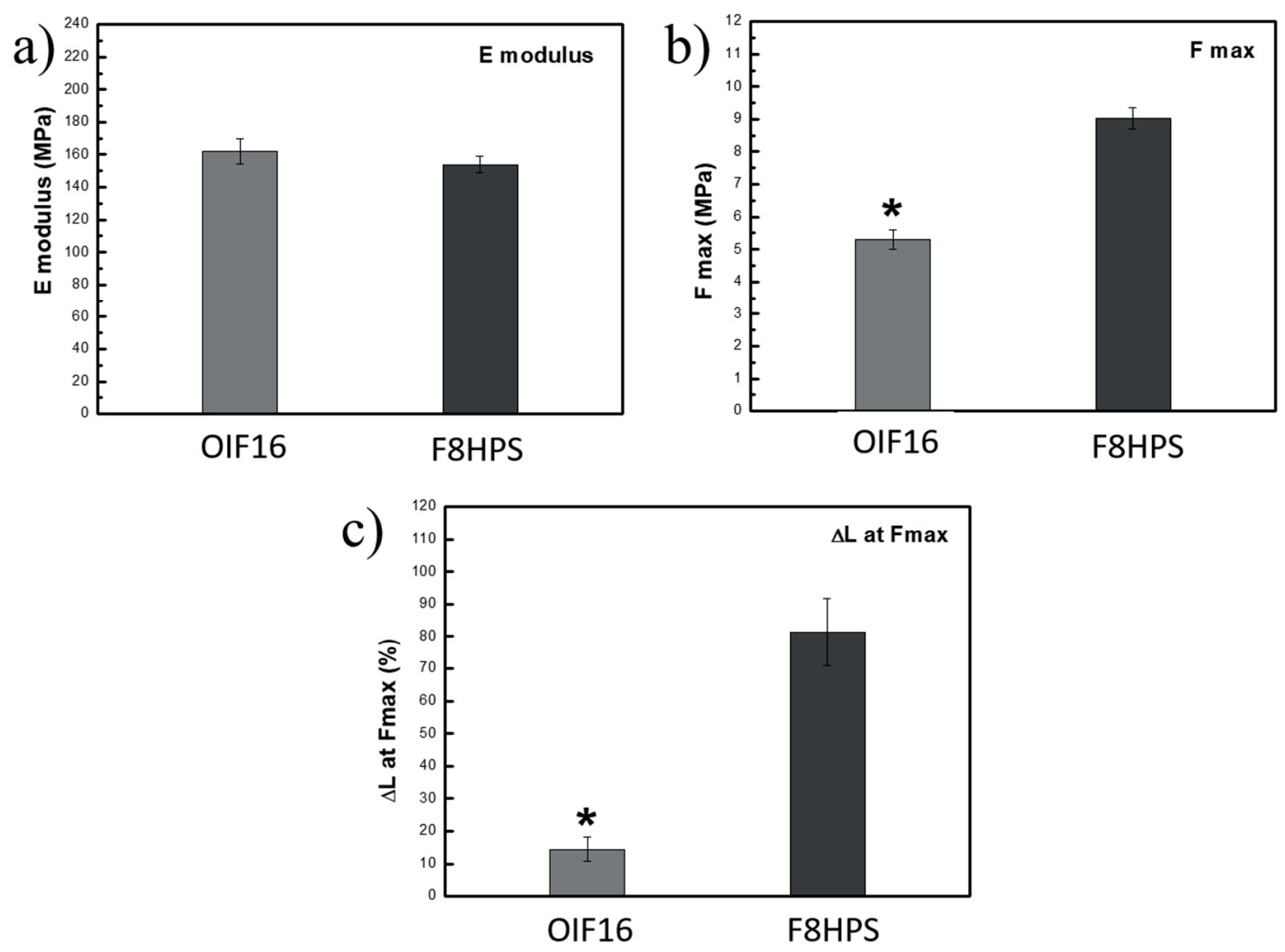

Figure 8. Mechanical test results of OIF16 and comparison to F8HPS. a) Young (E) modulus; b) Maximum strength before break; c) Maximum elongation before break. *: significant difference per $\mathrm{p}<0.05$.

\subsection{Comparison of OIF membranes developed here to literature}

This work presents a protocol for developing HD membranes for OIF (OIF16 has KUf $=15 \pm 3.5$ $\mathrm{mL} /\left(\mathrm{m}^{2} \cdot \mathrm{h} \cdot \mathrm{mmHg}\right)$ and albumin $\left.\mathrm{SC}=0.05 \pm 0.04\right)$, which has considerable advantages compared to other works. For example, Krause and co-inventors [13] also reported OIF fibers based on polysulfone, polyethersulphone (PES) or polyarylethersulphone and a blend of at least two homopolymers of polyvinylpyrrolidone (PVP) with KUf in the range $4.7-470 \mathrm{~mL} /\left(\mathrm{m}^{2} \cdot \mathrm{h} \cdot \mathrm{mmHg}\right)$. The 
described membranes have high diffusive transport (MWCO up to 100000 Dalton), thus are not suitable for HD applications. Besides, their manufacturing protocol is complex, including the heating of the spinneret and of the application of coagulation bath based on mixtures of solvent and water. In contrast, our protocol is simpler and eco-friendlier; it requires only water as non-solvent and the spinning takes place at room temperature. Gorsuch and co-inventors [14] reported OIF with KUf in the range $180-2400 \mathrm{~mL} /\left(\mathrm{m}^{2} \cdot \mathrm{h} \cdot \mathrm{mmHg}\right)$ and a plasma transmembrane flux in the range $3000-72000$ $\mathrm{mL} /\left(\mathrm{m}^{2} \cdot \mathrm{h} \cdot \mathrm{mmHg}\right)$, thus not being suitable for HD applications.

Another important advantage of the OIF16 developed here is the small fiber diameter / wall thickness compared to others described in literature. For example, Xu et al. [15] and Qin et al. [16] reported development of OIF fibers based on PES with high albumin retention and KUf in the range $61-217$ $\mathrm{mL} /\left(\mathrm{m}^{2} \cdot \mathrm{h} \cdot \mathrm{mmHg}\right)$ and $81-394 \mathrm{~mL} /\left(\mathrm{m}^{2} \cdot \mathrm{h} \cdot \mathrm{mmHg}\right)$, respectively. However, these fibers are quite large (inner diameter in the range of $550-720 \mu \mathrm{m}$ for Xu et al. [15] and $430-750 \mu \mathrm{m}$ for Qin et al. [16]) requiring application of large modules.

Finally, we believe that the protocol for OIF proposed here could be easily implemented to the current HD membrane production, since it uses PES and PVP, which are polymers already widely used for the production of HD membranes, and requires small tuning of spinning conditions that already applied for the manufacturing of standard inside-out HF.

\section{Conclusions and outlook}

In this work we developed an easy-to apply and eco-friendly protocol for the production of HF membranes for OIF. Our obtained HF has the selective hydrophilic layer on the outside of the fiber, high KUf and low albumin SC. Besides, the fiber diameter is small to achieve modules with high fiber packing density.

A range of spinning parameters was tuned in order to obtain the desired OIF fiber. By increasing the amount of solvent (NMP) in the bore solution, we could obtain fibers with more open structure at the 
lumen side. The decrease of the dope flow rate and bore flow rate led to thinner membrane walls and to smaller inner diameter of the fibers, respectively. Moreover, the increase of the take-up wheel led to a significant reduction of the membrane size. Finally, by decreasing the polymer dope concentration, fibers with high KUf and low albumin SC were obtained.

Long term studies with full blood will be performed in the future to investigate the hemocompatibility profile and the performance of the new OIF fibers. The reduction of thrombosis-derived clogging in OIF will also be assessed.

\section{Acknowledgments}

This work was conducted within the context of a research project funded by Novaflux, Inc., 1 Wall St., Princeton, NJ, 08540, USA. 


\section{References}

[1] S. Eloot, D. Schneditz, T. Cornelis, W. Van Biesen, G. Glorieux, A. Dhondt, J. Kooman, R. Vanholder, Protein-bound uremic toxin profiling as a tool to optimize hemodialysis, PLoS ONE 11(1) (2016) e0147159.

[2] M.P.M. Graham-Brown, D.R. Churchward, A.C. Smith, R.J. Baines, J.O. Burton, A 4-month programme of in-centre nocturnal haemodialysis was associated with improvements in patient outcomes, Clinical Kidney Journal 8(6) (2015) 789-795.

[3] M. Dam, P.J.M. Weijs, F.J. van Ittersum, B.C. van Jaarsveld, Physical performance in patients treated with nocturnal hemodialysis - a systematic review of the evidence, BMC Nephrology 20(1) (2019) 317.

[4] T. Cornelis, S. Eloot, R. Vanholder, G. Glorieux, F.M. van der Sande, J.L. Scheijen, K.M. Leunissen, J.P. Kooman, C.G. Schalkwijk, Protein-bound uraemic toxins, dicarbonyl stress and advanced glycation end products in conventional and extended haemodialysis and haemodiafiltration, Nephrology Dialysis Transplantation 30(8) (2015) 1395-1402.

[5] A. Davenport, Portable and wearable dialysis devices for the treatment of patients with end-stage kidney failure: wishful thinking or just over the horizon?, Pediatric Nephrology 30(12) (2015) 2053-2060.

[6] A. Davenport, V. Gura, C. Ronco, M. Beizai, C. Ezon, E. Rambod, A wearable haemodialysis device for patients with end-stage renal failure: a pilot study, The Lancet 370(9604) (2007) 2005-2010.

[7] S.S. Dukhin, Y. Tabani, R. Lai, O.A. Labib, A.L. Zydney, M.E. Labib, Outside-in hemofiltration for prolonged operation without clogging, Journal of Membrane Science 464 (2014) 173-178.

[8] S. Uchino, N. Fealy, I. Baldwin, H. Morimatsu, R. Bellomo, Continuous is not continuous: the incidence and impact of circuit "down-time" on uraemic control during continuous veno-venous haemofiltration, Intensive Care Medicine 29(4) (2003) 575-578.

[9] P.D. Brophy, M.J. Somers, M.A. Baum, J.M. Symons, N. McAfee, J.D. Fortenberry, K. Rogers, J. Barnett, D. Blowey, C. Baker, T.E. Bunchman, S.L. Goldstein, Multi-centre evaluation of anticoagulation in patients receiving continuous renal replacement therapy (CRRT), Nephrology Dialysis Transplantation 20(7) (2005) 1416-1421.

[10] R. Hofbauer, D. Moser, M. Frass, R. Oberbauer, A.D. Kaye, O. Wagner, S. Kapiotis, W. Druml, Effect of anticoagulation on blood membrane interactions during hemodialysis, Kidney International 56(4) (1999) 1578-1583.

[11] G. Catapano, A. Wodetzki, U. Baurmeister, Blood flow outside regularly spaced hollow fibers: the future concept of membrane devices?, The International Journal of Artificial Organs 15(6) (1992) 327-330. 
[12] E. Klein, The multidisciplinary approach to develop artificial organs, Blood Purification 21(4-5) (2003) 305-317.

[13] B. Kraus, H. Gohl, M. Hornung, C. Kraft, Hollow fiber membrane and method for manufacturing thereof, EP2083939B1, 2007.

[14] R.G. Gorsuch, H. Grage, H. Handley JR., H.W. Peters, J C. Kearns, Specialized hollow fiber membranes for plasmapheresis and ultrafiltration US 2007/0023353A1, 2007.

[15] Z.L. Xu, F. Alsalhy Qusay, Polyethersulfone (PES) hollow fiber ultrafiltration membranes prepared by PES/non-solvent/NMP solution, Journal of Membrane Science 233(1) (2004) 101-111.

[16] J.J. Qin, J. Gu, T.S. Chung, Effect of wet and dry-jet wet spinning on the shear-induced orientation during the formation of ultrafiltration hollow fiber membranes, Journal of Membrane Science 182(1) (2001) 57-75.

[17] M. Irfan, A. Idris, Overview of PES biocompatible/hemodialysis membranes: PES-blood interactions and modification techniques, Materials Science and Engineering: C 56 (2015) 574-592.

[18] Y. Liu, G.H. Koops, H. Strathmann, Characterization of morphology controlled polyethersulfone hollow fiber membranes by the addition of polyethylene glycol to the dope and bore liquid solution, Journal of Membrane Science 223(1) (2003) 187-199.

[19] X.M. Li, T. He, Does more solvent in bore liquid create more open inner surface in hollow fiber membranes?, Polymers for Advanced Technologies 19(7) (2008) 801-806.

[20] A.F. Ismail, M.I. Mustaffar, R.M. Illias, M.S. Abdullah, Effect of dope extrusion rate on morphology and performance of hollow fibers membrane for ultrafiltration, Separation and Purification Technology 49(1) (2006) 10-19.

[21] K. Kimmerle, H. Strathmann, Analysis of the structure-determining process of phase inversion membranes, Desalination 79(2) (1990) 283-302.

[22] J.J. Qin, T.S. Chung, Effects of orientation relaxation and bore fluid chemistry on morphology and performance of polyethersulfone hollow fibers for gas separation, Journal of Membrane Science 229(1) (2004) $1-9$.

[23] I.M. Wienk, F.H.A. Olde Scholtenhuis, T. van den Boomgaard, C.A. Smolders, Spinning of hollow fiber ultrafiltration membranes from a polymer blend, Journal of Membrane Science 106(3) (1995) 233-243.

[24] C. Barth, M.C. Gonçalves, A.T.N. Pires, J. Roeder, B.A. Wolf, Asymmetric polysulfone and polyethersulfone membranes: effects of thermodynamic conditions during formation on their performance, Journal of Membrane Science 169(2) (2000) 287-299. 
[25] T.S. Chung, Z.L. Xu, W. Lin, Fundamental understanding of the effect of air-gap distance on the fabrication of hollow fiber membranes, Journal of Applied Polymer Science 72(3) (1999) 379-395.

[26] O.E.M. ter Beek, D. Pavlenko, D. Stamatialis, Hollow fiber membranes for long-term hemodialysis based on polyethersulfone-SlipSkin ${ }^{\mathrm{TM}}$ polymer blends, Journal of Membrane Science 604 (2020) 118068.

[27] D. Kim, D. Stamatialis, High flux mixed matrix membrane with low albumin leakage for blood plasma detoxification, Journal of Membrane Science 609 (2020) 118187.

[28] M.S.L. Tijink, M. Wester, G. Glorieux, K.G.F. Gerritsen, J. Sun, P.C. Swart, Z. Borneman, M. Wessling, R. Vanholder, J.A. Joles, D. Stamatialis, Mixed matrix hollow fiber membranes for removal of protein-bound toxins from human plasma, Biomaterials 34(32) (2013) 7819-7828.

[29] G. Catapano, J. Vienken, Biomedical applications of membranes, Advanced Membrane Technology and Applications, John Wiley \& Sons, 2008, 489-517.

[30] Fresenius, F-Series, 2020. https://www.freseniusmedicalcare.asia/en/healthcareprofessionals/hemodialysis/dialyzers/f-series-dialyzers/. (Accessed 9 August 2020).

[31] M. Kohlova, C.G. Amorim, A. Araujo, A. Santos-Silva, P. Solich, M. Montenegro, The biocompatibility and bioactivity of hemodialysis membranes: their impact in end-stage renal disease, Journal of Artificial Organs 22(1) (2019) 14-28.

[32] F. Ran, X. Niu, H. Song, C. Cheng, W. Zhao, S. Nie, L. Wang, A. Yang, S. Sun, C. Zhao, Toward a highly hemocompatible membrane for blood purification via a physical blend of miscible comb-like amphiphilic copolymers, Biomaterials Science 2(4) (2014) 538-547.

[33] N. Cai, Q. Li, J. Zhang, T. Xu, W. Zhao, J. Yang, L. Zhang, Antifouling zwitterionic hydrogel coating improves hemocompatibility of activated carbon hemoadsorbent, Journal of Colloid and Interface Science 503 (2017) 168-177. 


\section{Supplementary Information}

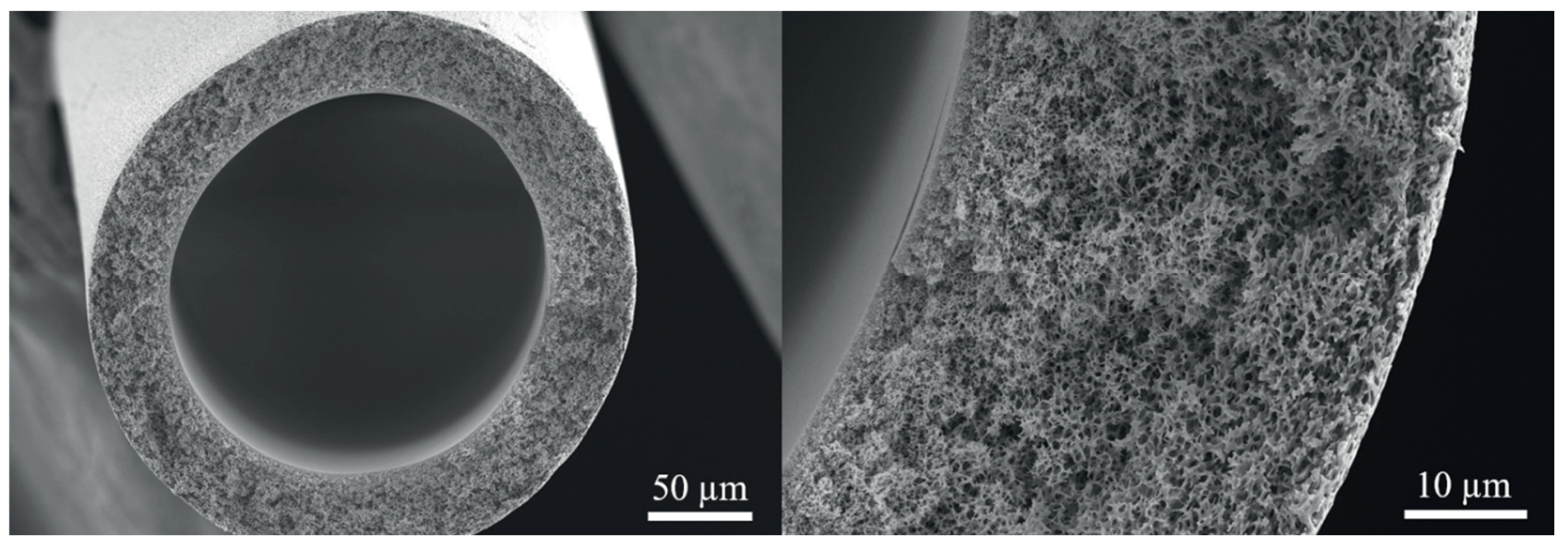

Figure S1. Overall cross section of F8HPS fiber (left) and magnification of the wall (right).

F8HPS Fresenius fiber presents a spongy structure with the selective layer at the lumen side and a porous outer surface. 



\section{Chapter 5}

\section{New mixed matrix membrane for the removal of urea from dialysate solution}

I. Geremia $^{\text {a }}$, J.A.W. Jong ${ }^{\text {b }}$, C.F. van Nostrum ${ }^{\text {b }}$, W.E. Hennink ${ }^{\text {b }}$, K.G.F. Gerritsen ${ }^{\text {c }}$, D. Stamatialis ${ }^{a}$

a (Bio)artificial organs, Department of Biomaterials Science and Technology, TechMed Centre, Faculty of Science and Technology, University of Twente, P.O. Box 217, 7500 AE Enschede, The Netherlands

${ }^{\mathrm{b}}$ Department of Pharmaceutics, Utrecht Institute for Pharmaceutical Sciences (UIPS), Utrecht University, Universiteitsweg 99, 3584 CG Utrecht, The Netherlands

${ }^{c}$ Department of Nephrology and Hypertension, University Medical Center Utrecht, Heidelberglaan 100, 3584 CX Utrecht, The Netherlands 


\section{Abstract}

Urea is the main waste product of nitrogen metabolism in mammals and is excreted into the urine. During hemodialysis, urea is removed from the plasma of the end stage kidney disease patients to the dialysate. Portable and wearable artificial kidneys (WAK) are based on continuous regeneration of a small volume of spent dialysate so that it can be reused in a closed loop. Urea removal from spent dialysate is one of the biggest challenges in dialysate regeneration since urea is a molecule with low reactivity that is difficult to adsorb while it is the waste solute with the highest daily molar production. In fact, several methods have been proposed for its removal, but they have not found clinical implementation mainly due to their limited efficiency. Carbonyl-type sorbents have high urea binding capacity; however, so far, they were studied for urea removal only in packed bed columns, showing slow binding kinetics and rather poor particle accessibility. In this work, we investigate the application of a new Mixed Matrix Membrane (MMM) for urea removal consisting of polystyrenebased ninhydrin particles, an already known carbonyl-type sorbent for urea, within a polyethersulfone/polyvinylpyrrolidone polymer blend matrix. Thanks to the good dispersion of small size particles, the MMM removes under static conditions, at $70^{\circ} \mathrm{C}, 2.1 \pm 0.1 \mathrm{mmol}$ of urea per grams of particles at 24 hours, while urea removal by the particles in suspension reaches $1.7 \pm 0.1 \mathrm{mmol} / \mathrm{g}$ under the same conditions. Importantly, in dynamic experiments (continuous recirculation), performed at $70{ }^{\circ} \mathrm{C}$ using a laboratory scale module, the MMM removes $3.4 \pm 0.3 \mathrm{mmol}$ of urea per grams of particles. Based on these results we can estimate that 215 grams of MMM are needed for removing the daily produced urea from spent dialysate. These results are very promising for application to portable and/or WAK, where miniaturization and lightweight are required. 


\section{Introduction}

Hemodialysis (HD) treatment is a life-sustaining therapy for the treatment of end stage kidney disease (ESKD) patients waiting for or not being suitable for kidney transplantation. However, due to the intermittent treatment performed mostly in the clinics 4 hours - 3 times per week, accumulation of toxins and excess water occurs in between dialysis sessions [1, 2].

Portable (PAK) and wearable artificial kidney (WAK) devices have been proposed to improve blood purification as well as patients' quality of life. These systems would provide continuous or semicontinuous blood purification, mimicking closer the natural kidneys, leading to higher clearance of toxins and higher removal of excess water $[3,4]$. Moreover, they would allow for dialysis at home, improving patients' mobility and participation in social life and would significantly lower the amount of water needed for the treatment [5-7]. In fact, for a standard HD session of 4 hours $280-500 \mathrm{~L}$ of water is used to generate $120 \mathrm{~L}$ of pure dialysate $[8,9]$. In contrast, for a PAK and/or WAK a small volume of spent dialysate (preferably $0.5 \mathrm{~L}$ or lower) would be continuously regenerated and recirculated to guarantee portability and wearability $[6,7]$. In order to regenerate the spent dialysate, ions such as phosphate and potassium, small organic waste solutes such as creatinine and urea and middle molecules such as $\beta 2$-microglobulin need to be removed [10]. Ion-exchangers can be used for the removal of phosphate and potassium, while activated carbon can efficiently remove most organic waste solutes $[3,5,10,11]$. However, the biggest challenge is the removal of urea [10] which is the main waste product of nitrogen metabolism (waste solute with the highest daily molar production of 240 to $470 \mathrm{mmol}$, depending on protein intake) $[12,13]$. High urea plasma concentrations (in the range $20-30 \mathrm{mM}$, as for the ESKD patients) are associated with toxicity, including insulin resistance, disruption of the gastrointestinal barrier, production of radical oxygen species and endothelial changes promoting atherosclerosis [14]. Urea removal from spent dialysate is difficult because of its small molecular weight $(\mathrm{MW}=60 \mathrm{~g} / \mathrm{mol})$, its chemical similarity to water and because of the absence of charges at physiologic $\mathrm{pH}$, which does not allow for the formation of ionic bonds $[10,15]$. 
Many strategies have been proposed for its removal from spent dialysate. For example, enzymatic decomposition of urea by means of ureases is very efficient and was applied in the REDY (Recirculation of DialYsis) device. However, this system was withdrawn from the market because of production of toxic byproducts, high costs and lower efficiency compared to single-pass HD [16-20]. Electrooxidation of urea, which converts urea in nitrogen, carbon dioxide and hydrogen gases, is quite efficient too. The formed gases can be easily removed by a bubble trap; nevertheless, toxic side products, as nitrate, nitrite, ammonia, chloramines and active chlorine species are formed [10, 21-24]. Several sorbents have also been proposed for the removal of urea from spent dialysate via physisorption (non-covalent binding), coordination or covalent binding (chemisorption) [10]. Activated carbon [25-28], silica [29], zeolites [25, 30] and MXenes nanosheets were used for urea removal via physisorption [31]. However, they have rather low affinity for urea in water because they rely on hydrogen bonding, van der Waals and dipole interactions [10]. Another strategy for urea removal is the use of chitosan complexed with copper ions [32-35]. Although various studies reported high urea binding capacity, the toxicity derived by potential copper leaching is a concern [10]. Several other studies report the synthesis of urea-molecular imprinted polymers for urea detection and/or removal [36-39]. There, competition by other dialysate components for binding is avoided. However, as for the work of Alizadeh et al. [37], many questions arise from the high urea binding capacity compared to the much lower theoretical maximum urea binding capacity of the imprinted polymer [10]. Carbonyl-type sorbents, although not selective towards urea, possess high urea binding capacity, are not toxic when immobilized in the dialysate circuit and can form irreversible covalent bond between the electrophilic carbonyl groups and weakly nucleophilic urea molecules [40]. Several sorbents containing urea-reactive carbonyl groups have been reported in literature, such as aldehydes, $\alpha$-ketoaldehyde hydrates, ninhydrins, $\alpha$-ketoesters and glyoxaldehydes [41-50]. The urea binding is slow, but it can increase at higher temperatures $[10,41,50,51]$. Thus far, carbonyl-type sorbents have been investigated for urea removal only in suspension or in bed column systems [41-48, 51, 52], 
where high pressure drop, rather bad particle dispersion and aggregation could limit the amount of available binding sites, thus reducing their removal capacity.

In earlier studies, we have shown that the application of a Mixed Matrix Membrane (MMM), consisting of activated carbon sorbent particles embedded within a polyethersulphone (PES)/polyvinylpyrrolidone (PVP) polymer matrix, can remove a high amount of uremic toxins from blood plasma. The optimal distribution of small sorbent particles in the MMM guarantees optimal particle accessibility leading to high toxins removal [53-55]. Here, we investigate the application of a new MMM hollow fiber (HF) for urea removal from dialysate solution. This MMM consists of a carbonyl-type sorbent that contains reactive ninhydrin groups and is prepared from polystyrene particles (PS-Nin particles) embedded within a PES/PVP polymer matrix. The synthesis of the PSNin particles and their application for urea removal from dialysis fluid have been reported elsewhere $[48,51]$. They have quite high theoretical urea binding capacity of approximately $2.7 \mathrm{mmol} / \mathrm{g}$, considering that approximately $55 \%$ of the phenyl groups of polystyrene are transformed in ninhydrin units, as assessed by Smakman et al. $[48,51]$. Moreover, this material can be sterilized, do not leach any compounds into the dialysate and have been even suggested for oral use [51]. Here, we hypothesize that the optimal dispersion of small size PS-Nin sorbent particles within the MMM would minimize particle aggregation and lead to high particle accessibility and therefore high urea removal. The MMM hollow fiber is produced via dry-wet spinning technique and is characterized in terms of morphology (via scanning electron microscopy) and transport properties (clean water flux). The effects of sorbent particle size, temperature and incorporation in the polymer matrix on urea removal are first studied in static experiments. PES/PVP HF (without particles) is used as control to verify that the embedded PS-Nin particles are mainly responsible for urea removal. Finally, dynamic urea removal experiments, where a small volume of dialysate model solution spiked with urea is recirculated for 4 hours through the MMM HF, are performed to assess the urea removal by the MMM under conditions better mimicking PAK or WAK systems. 


\section{Materials and methods}

\subsection{Polystyrene-ninhydrin beads (PS-Nin beads) characterization}

\subsubsection{Polystyrene-ninhydrin (PS-Nin) beads}

Polystyrene beads containing indanone groups (PS-Ind beads), synthesized as described by Smakman et al. $[48,51]$, were obtained from Innovista (Nigtevecht, the Netherlands). The indanone groups in the particles were oxidized into ninhydrin groups using a method described previously $\left(90{ }^{\circ} \mathrm{C}, 1.0\right.$ eq. iodine and hydrogen iodide, 24 hours) [50]. These particles have theoretical urea binding capacity of approximately $2.7 \mathrm{mmol} / \mathrm{g}$, considering that the predicted amount of phenyl groups of polystyrene transformed in ninhydrin units is $55 \%$, as reported by Smakman et al. [48, 51].

\subsubsection{Optical microscopy}

The diameter of the sorbent beads was measured using a size calibrated Nikon eclipse TE2000-U optical microscope equipped with a digital camera (Nikon DS-2Mv camera and Nikon DS-U1 digital adapter, with a $4 \times$ magnification) and the NIS-elements basic research software package. Pictures of the beads were taken in the dry state and for 30 random beads 3 points on the perimeter of the beads were identified to allow calculation of the circular diameter by the program [56].

\subsubsection{Fourier Transform Infrared Spectroscopy (FTIR)}

Infrared (IR) spectra of the PS-Nin beads before and after reaction with urea were recorded neat using a Perkin Elkmer ATRU Spectrum 2. For the urea binding, the sorbent beads (50 mg per vial) were incubated for 24 hours at $70{ }^{\circ} \mathrm{C}$ with a urea solution $(5.0 \mathrm{~mL}, 50 \mathrm{mM})$ in Phosphate Buffer Saline (PBS) in two glass vials, after which the beads were allowed to settle and the urea concentration in the supernatant was determined. All scans were performed at room temperature at a resolution of 1 $\mathrm{cm}^{-1}$. 


\subsubsection{Brunauer-Emmett-Teller (BET) surface area measurement of PS-Nin beads}

$\mathrm{N}_{2}$ sorption isotherms were measured at $77 \mathrm{~K}$ using Micromeritics TriStar 3000 and TriStar II Plus apparatus. Prior to this, the samples were dried overnight under vacuum at room temperature. Surface areas were determined using the Brunauer-Emmett-Teller (BET) method, the micropore $(<2 \mathrm{~nm})$ volumes were based on a t-plot analysis using a Harkins-Jura thickness curve and the total pore volumes were derived from the amount of $\mathrm{N}_{2}$ adsorbed at $\mathrm{p} / \mathrm{p}_{0}=0.995$ [57].

\subsubsection{Scanning Electron Microscopy (SEM)}

PS-Nin beads were imaged using a scanning electron microscope (SEM, Phenom, FEI Company, The Netherlands). Dried beads were transferred onto $12 \mathrm{~mm}$ diameter aluminum specimen stubs (Agar Scientific Ltd., England) using double-sided adhesive tape. Prior to SEM analysis, the beads were coated with platinum using an ion coater under vacuum.

\subsection{Membrane fabrication}

Prior to membrane fabrication, PS-Nin beads were grinded using a mortar and pestle. Afterwards, the grinded PS-Nin particles were sieved through a $63 \mu \mathrm{m}$ sieve. For better clarity, throughout the manuscript, "PS-Nin beads" is used to indicate the large polystyrene-ninhydrin beads before grinding and sieving and "PS-Nin particles" indicates the small polystyrene-ninhydrin particles obtained after grinding and sieving.

The HF MMM was prepared by incorporating PS-Nin particles within PES/PVP polymer matrix. The HF MMM was produced via dry-wet spinning technique. The polymer dope solution was prepared by dissolving Ultrason E6020 PES (BASF, Ludwigshafen, Germany) and PVP K90 (MW ₹ 360 kDa, Sigma-Aldrich Chemie GmbH, Munchen, Germany) in ultrapure N-methylpyrrolidone (NMP) (Acros Organics, Geel, Belgium). PS-Nin particles were added to the dope solution in order to have a final weight of particles equal to $55 \%$ of the dried weight of the membrane. The PES/PVP/PS-Nin particles mixture was stirred for two days at $60{ }^{\circ} \mathrm{C}$ to ensure well-dispersion of the particles in the 
polymer solution; afterwards it was transferred in stainless-steel syringes and left to degas for 24 hours. The concentrations of PES, PVP and PS-Nin particles and the spinning parameters used in the study are specified in Table 1. After degassing, the syringe was connected to a high-pressure syringe pump and to a designed spinneret for preparing the HF (specifications given in Table 1). Ultrapure water was used as bore forming solution. The airgap between the spinneret and the coagulation bath was adjusted to $5.5 \mathrm{~cm}$. The HF was left to free-fall in the water coagulation bath. The fabricated membrane was washed with demineralized-water and stored in demineralized-water for further use.

Table 1. Specifications of the spinning parameters used for the fabrication of the MMM.

\begin{tabular}{|l|l|}
\hline Dope composition (PES/PVP/PS-Nin Particles/NMP) & $6.6 / 3.1 / 11.9 / 78.4 \mathrm{wt} \%$ \\
\hline Dope pumping speed & $1 \mathrm{~mL} / \mathrm{min}$ \\
\hline Spinneret - Thickness dope orifice & $0.6 \mathrm{~mm}$ \\
\hline Bore liquid & Ultrapure water \\
\hline Bore liquid pumping speed & $1 \mathrm{~mL} / \mathrm{min}$ \\
\hline Spinneret - Diameter bore needle & $1.35 \mathrm{~mm}$ \\
\hline Air gap & $5.5 \mathrm{~cm}$ \\
\hline Coagulation bath composition & Ultrapure water \\
\hline Fibers collection & Free falling \\
\hline
\end{tabular}

\subsection{Scanning Electron Microscopy (SEM)}

The morphology of the HF MMM and of the PS-Nin particles after grinding and sieving was analyzed by SEM (JEOL JSM-IT 100, Tokyo, Japan). Membrane samples were dried in air and fractured in liquid nitrogen for the imaging of the cross-sections. Prior to SEM imaging, the samples were gold sputtered using the Cressington 108 auto sputter (Cressington Scientific Instruments, Watford, UK). 


\subsection{Water transport experiment}

Membrane modules composed of $3 \mathrm{HF}$ with a total surface area of $13.9 \pm 0.1 \mathrm{~cm}^{2}$ were used. A 2compontent epoxy glue (Griffon Combi Snel-Rapide, Bison International, Goes, The Netherlands) was used for the preparation of the modules. Before water transport experiments, the HF modules (n =6) were pre-wetted with EtOH for 30 minutes at a trans-membrane pressure (TMP) of 0.2 Bar and pre-compacted with ultrapure water at a TMP of 0.6 Bar for 30 minutes. Afterwards, the amount of permeated water was measured over time at TMP of $0.2,0.4$ and 0.6 Bar. The resulting water permeance was calculated as the slope of the linear fit of the flux $\left(\mathrm{L} /\left(\mathrm{m}^{2} \cdot \mathrm{h}\right)\right)$ versus the TMP $($ Bar $)$.

\subsection{Static urea removal studies}

\subsubsection{Effect of temperature on urea removal kinetics on PS-Nin beads}

Urea removal experiments were performed at $37{ }^{\circ} \mathrm{C}, 50{ }^{\circ} \mathrm{C}$ and $70{ }^{\circ} \mathrm{C}$ in order to study the effect of temperature on the binding reaction of urea with the ninhydrin moiety in PS-Nin beads. The sorbent PS-Nin beads $(15 \mathrm{mg})$ were incubated in urea (Sigma-Aldrich, Zwijndrecht, The Netherlands) solution $(1.5 \mathrm{~mL}, 30 \mathrm{mM})$ in PBS ( $\mathrm{pH} 7.4)$. The samples $(\mathrm{n}=3$ for each time point $)$ were placed in an oven on a rotating device at 37,50 or $70^{\circ} \mathrm{C}$. After $1,2,4,8,16$ and 24 hours, the urea concentration in the supernatants was determined as described in section 2.7.

\subsubsection{Effect of particle size on urea removal kinetics}

In order to study the effect of the particle size on urea removal, kinetic urea removal experiments were performed at $70{ }^{\circ} \mathrm{C}$ on PS-Nin beads (before grinding and sieving) and on the PS-Nin particles after grinding and sieving (63 $\mu \mathrm{m}$ sieve). Both PS-Nin beads and PS-Nin particles (15 mg, each) were incubated in urea solution $(1.5 \mathrm{~mL}, 30 \mathrm{mM})$ in PBS ( $\mathrm{pH} 7.4)$. The samples $(\mathrm{n}=3$ for each time point) were shaken at $70{ }^{\circ} \mathrm{C}$ in a water bath and after $1,2,4,8,16$ and 24 hours the supernatants were 
collected via filtration. Urea concentration in the supernatant was determined as described in section 2.7 .

\subsubsection{Effect of incorporation of PS-Nin particles $(<63 \mu \mathrm{m})$ in the MMM}

In order to study the effect of the incorporation of the PS-Nin particles in the polymer matrix of the MMM, a urea removal experiment was performed at $70{ }^{\circ} \mathrm{C}$ with the MMM containing grinded and sieved particles. Results were compared with the urea removal kinetics of the grinded and sieved particles alone (see 2.5.2). The MMM (27 mg containing $15 \mathrm{mg}$ of embedded particles, $\mathrm{n}=3$ ) was incubated in dialysate model solution $(1.3 \mathrm{~mL})$, consisting of urea $(30 \mathrm{mM}), 2 \mathrm{mM} \mathrm{KCl}, 140 \mathrm{mM}$ $\mathrm{NaCl}, 1.5 \mathrm{mM} \mathrm{CaCl}_{2}, 0.25 \mathrm{mM} \mathrm{MgCl}, 35 \mathrm{mM} \mathrm{NaHCO}_{3}$ (all from Sigma-Aldrich Chemie $\mathrm{GmbH}$, Schnelldorf, Germany) and $5.5 \mathrm{mM}$ glucose (Life Technologies Europe BV, Bleiswijk, The Netherlands) in ultrapure water at $\mathrm{pH} 7.4$ and shaken at $70{ }^{\circ} \mathrm{C}$ in a water bath. After $1,2,4,8,16$ and 24 hours, the supernatants were collected (via filtration for the particles) and the urea concentration was determined as described in section 2.7.

\subsubsection{Urea binding isotherm of the MMM HF and PES/PVP control HF.}

The binding of urea by the MMM and a PES/PVP control HF was measured at various concentrations. PES/PVP control HF was prepared via dry-wet spinning technique as described by Geremia et al. [55]. Briefly, the polymer dope solution was prepared by dissolving Ultrason E6020 PES (15 wt\%) (BASF, Ludwigshafen, Germany) and PVP K90 (7 wt\%) (MW $\approx 360 \mathrm{kDa}$, Sigma-Aldrich Chemie $\mathrm{GmbH}$, Munchen, Germany) in ultrapure N-methylpyrrolidone (NMP) (78 wt\%) (Acros Organics, Geel, Belgium). The polymer solution was mixed on a roller bench for 3 days, then it was transferred into a stainless-steel syringe and left to degas for 24 hours. Afterwards, the syringe was connected to a high-pressure syringe pump and to a designed spinneret. The dope solution pumping speed was set at $0.4 \mathrm{~mL} / \mathrm{min}$. Ultrapure water was used as bore forming solution and the bore solution pumping speed was set at $1.2 \mathrm{~mL} / \mathrm{min}$. The airgap between the spinneret and the coagulation bath was adjusted 
to $10 \mathrm{~cm}$. A collecting wheel (speed $8.3 \mathrm{~m} / \mathrm{min}$ ) was used for the collection of the produced HF. The fabricated membrane was washed with demineralized-water and stored for further use. $27 \mathrm{mg}$ of the MMM (15 mg of particles in the MMM, $n=3)$ and $27 \mathrm{mg}$ of the PES/PVP control HF $(\mathrm{n}=3)$ were incubated in Eppendorf tubes in $1.5 \mathrm{~mL}$ dialysate model solution ( $\mathrm{pH}$ 7.4) spiked with urea at different concentrations. The samples were placed in a horizontally shaking water bath at $70{ }^{\circ} \mathrm{C}$. After 24 hours, the supernatants were collected and the urea concentration in the supernatants was determined as described in section 2.7 .

\subsection{Dynamic urea removal by the MMM}

Membrane modules composed of $3 \mathrm{HF}$ with a total surface area of $23.3 \pm 0.4 \mathrm{~cm}^{2}$ and with a total amount of particles embedded in the PES/PVP matrix equal to $77 \mathrm{mg}$ were used to study urea removal in dynamic conditions. A 2-compontent epoxy glue (Griffon Combi Snel-Rapide, Bison International, Goes, The Netherlands) was used for the preparation of the modules. Before dynamic urea removal experiments, the HF modules $(n=5)$ were kept in demineralized-water. Urea dynamic experiments were performed in filtration mode $(\mathrm{TMP}=0.15 \mathrm{Bar})$ with $30 \mathrm{mM}$ urea in a dialysate model solution continuously recirculated through the fibers at a flow rate of $20 \mathrm{~mL} / \mathrm{min}$ using a dedicated set up (Convergence, Enschede, The Netherlands). The removal experiments were performed at $70^{\circ} \mathrm{C}$ for 4 hours. In order to maintain a temperature of the urea solution of $70^{\circ} \mathrm{C}$ inside the recirculation system, the feed solution was heated at $70{ }^{\circ} \mathrm{C}$, the tubing was insulated and the HF module was immerged in a water bath heated at $90{ }^{\circ} \mathrm{C}$. In that way, the temperature within the module was kept as $70{ }^{\circ} \mathrm{C}$. This was confirmed by measuring the temperature of the urea solution at the exit of the module. Samples of the urea solution were collected every hour for quantification. At the end of the removal experiment, the HF module was removed from the water bath and was totally emptied. $24.5 \mathrm{~mL}$ of ultrapure water at room temperature were recirculated through the module at a flow rate of $20 \mathrm{~mL} / \mathrm{min}$ for 1 hour in order to elute unbound or loosely bound urea from the MMM. Urea concentration was determined as described in section 2.7 . 


\subsection{Quantification of urea concentration}

For the urea removal experiments with the PS-Nin particles, the MMM and the PES/PVP control HF, urea concentration was determined by the enzymatic assay Urea FS* (Diasys, Holzheim, Germany). For the urea removal experiments with the PS-Nin beads, an AU 5800 routine chemistry analyzer (Beckman Coulter, Brea, CA) for the determination of urea concentration was used. Both methods are based on a coupled enzyme reaction, which results in a colorimetric product proportional to the urea concentration. Via the mass balance, the amount of urea adsorbed was calculated from the depleted amount of urea in the solution. 


\section{Results and discussion}

\subsection{PS-Nin beads and PS-Nin particles characterization}

\subsubsection{Optical microscopy and Scanning Electron Microscopy (SEM) of the PS-Nin beads and}

particles

Figure 1 shows the pictures of the PS-Nin beads before grinding and sieving (Fig. 1a) and of the PS-

Nin particles after grinding and sieving with the mortar and pestle (Fig. 1b). Pictures of the 30 randomly selected PS-Nin beads were taken, before grinding and sieving, in the dry state. Three points on the perimeter of the beads were identified to allow calculation of circular diameter by the program [56]. The average diameter \pm standard deviation of PS-Nin beads was $483 \pm 282 \mu \mathrm{m}$.

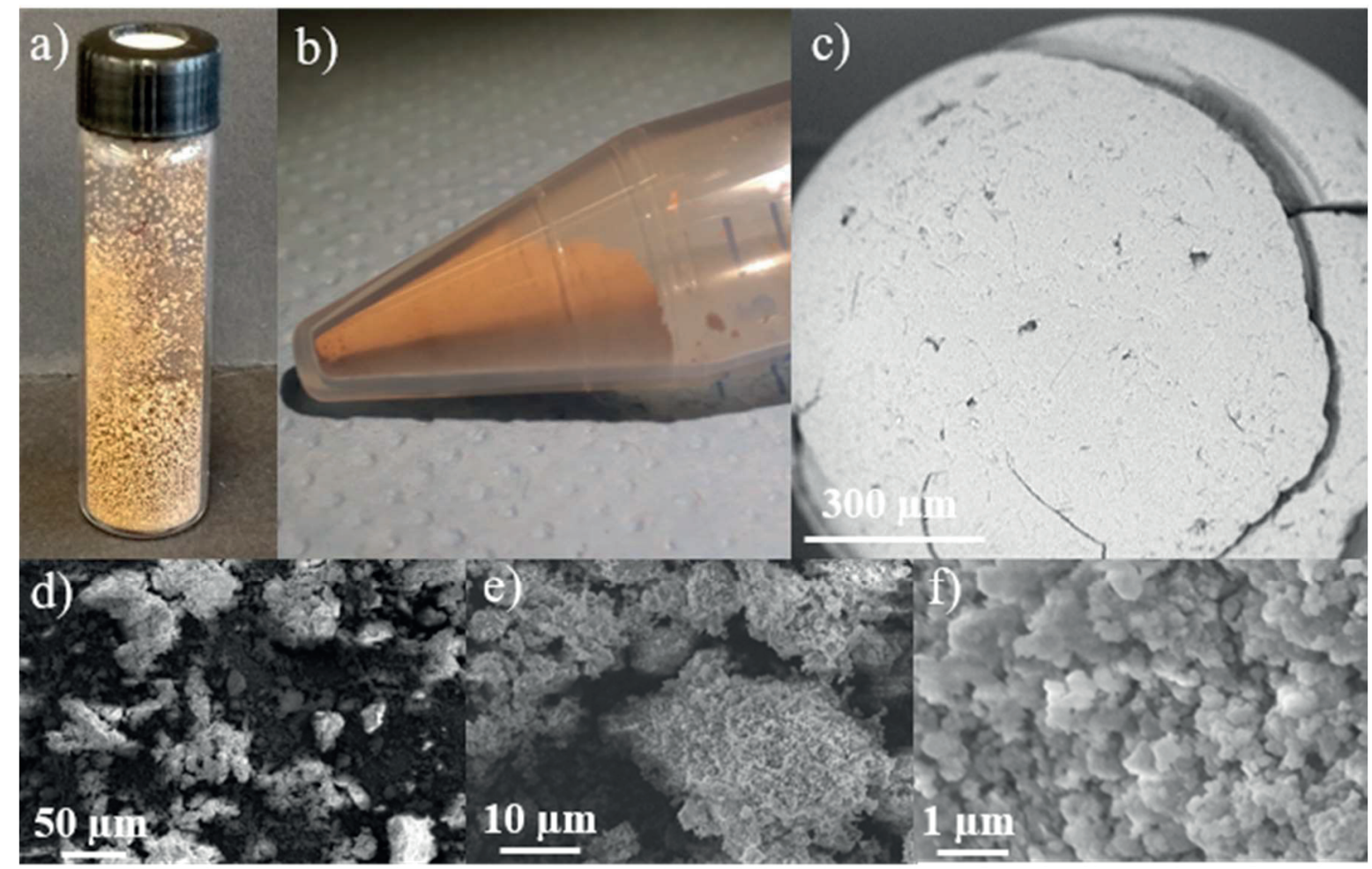

Figure 1. Pictures of the PS-Nin beads before grinding and sieving (a) and of the PS-Nin particles after grinding and sieving (b). SEM images of the PS-Nin beads before grinding and sieving (c) and of the PS-Nin particles after grinding and sieving at different magnifications: d) 370X, e) 2300X, f) 20000X.

SEM imaging of the PS-Nin particles after grinding and sieving (Fig. $1 \mathrm{~d}$, e, f) reveals that the particles are characterized by a granular morphology, where the biggest particles are composed by stable aggregates of submicron particles. 


\subsubsection{FTIR of PS-Nin beads before and after binding of urea}

In order to confirm the oxidation of the indanone groups to ninhydrin groups, the sorbent PS-Nin beads were characterized by means of FTIR spectroscopy and compared to the FTIR spectrum of pure ninhydrin (Fig. 2a). The PS-Nin beads spectrum is characterized by the peaks corresponding to the stretching vibrations of the $\mathrm{C}=\mathrm{O}$ bonds $\left(1700\right.$ and $\left.1750 \mathrm{~cm}^{-1}\right)$, the aromatic $\mathrm{C}=\mathrm{C}$ bonds $(1600$ $\left.\mathrm{cm}^{-1}\right)$ and the $\mathrm{C}-\mathrm{O}$ bonds $\left(1090 \mathrm{~cm}^{-1}\right)$ confirming the presence of ninhydrin groups in the oxidised sorbent beads, as already reported by Jong et al. for very similar ninhydrin-type sorbent [50].

a)

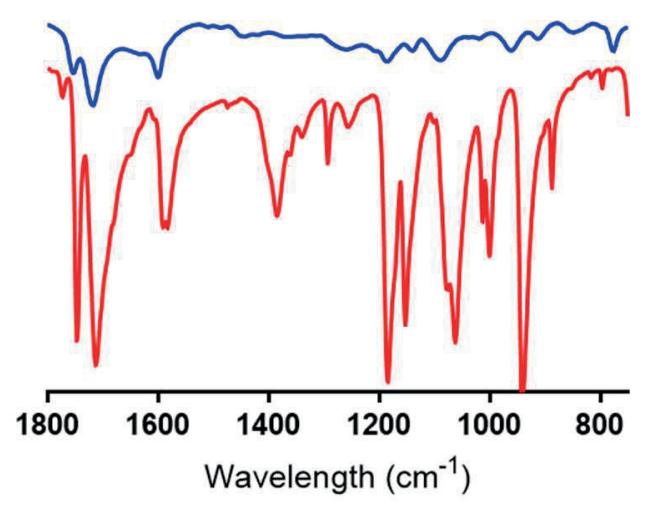

- PS-Nin

- Ninhydrin
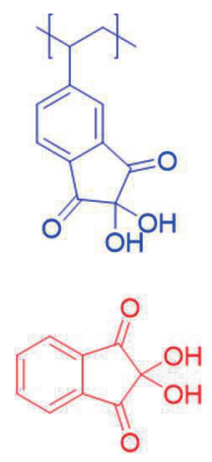

b)
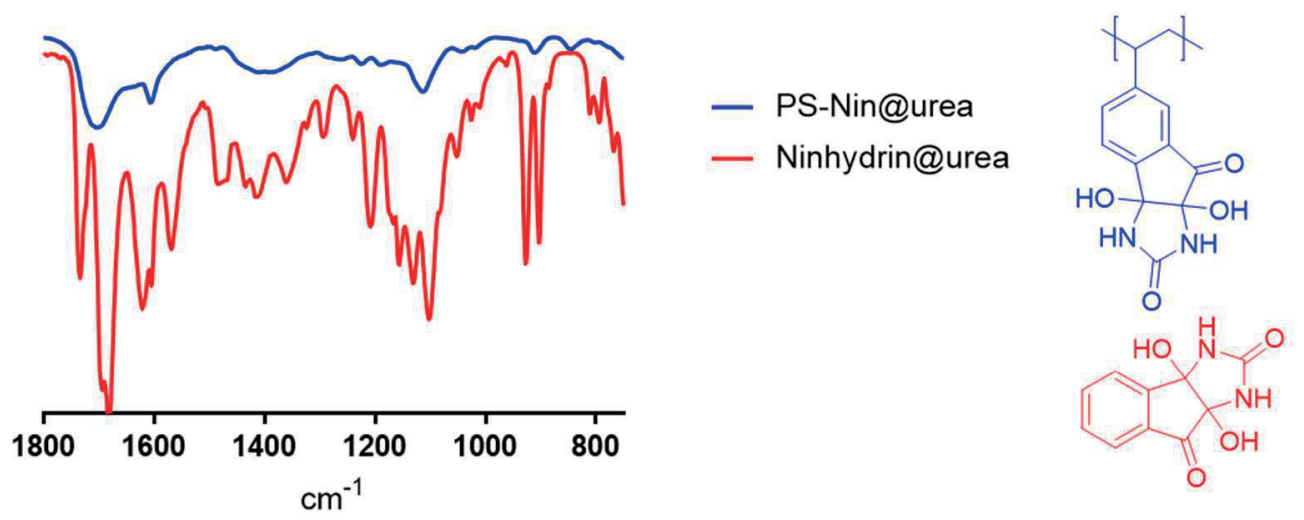

c)<smiles>[R]c1ccc2c(c1)C(=O)C(O)(O)C2=O</smiles>
and<smiles>[R]c1ccc2c(c1)C1(O)NC(=O)NC1(O)C2=O</smiles>

Figure 2. a) Infrared spectra of ninhydrin and PS-Nin beads; b) Infrared spectra of the reaction product of ninhydrin and urea (Ninhydrin@urea) and PS-Nin beads after reaction with urea (PS-Nin@urea); c) Reaction scheme of the addition of urea to ninhydrin. 
The chemical reaction between ninhydrin and urea is shown in Fig. 2c [58]. Ninhydrin has the ability to form an irreversible covalent bond between its electrophilic carbonyl group and the weakly nucleophilic urea molecule. A hemiaminal is the result of the nucleophilic attack of the electron pair on the nitrogen atom of urea to the ninhydrin carbonyl groups. In order to investigate the ability of the ninhydrin groups on the PS-Nin beads to bind urea, we compared the infrared spectra of the PSNin beads after reaction with urea with pure ninhydrin-urea complex (Fig. 2b). Both for the PS-Nin beads after reaction with urea and for pure ninhydrin-urea complex it is possible to observe the presence of characteristic peaks of urea, as the peak at $1680 \mathrm{~cm}^{-1}$ corresponding to the $\mathrm{C}=\mathrm{O}$ symmetric stretching, the peak at $1420 \mathrm{~cm}^{-1}$ corresponding to the $\mathrm{N}-\mathrm{C}-\mathrm{N}$ asymmetric stretching and the peak at $1110 \mathrm{~cm}^{-1}$ corresponding to the C-N stretching $[59,60]$, thus confirming the ability of the PS-Nin beads to covalently bind urea.

\subsubsection{Brunauer-Emmett-Teller (BET) surface area measurement of PS-Nin beads}

The surface area (SBET) and pore volume of the PS-Nin beads as determined by nitrogen physisorption are $23.7 \mathrm{~m}^{2} / \mathrm{g}$ and $0.14 \mathrm{~mL} / \mathrm{g}$, respectively, which are quite low. For comparison, the SBET of Norit A Supra activated carbon (AC) is $1700 \mathrm{~m}^{2} / \mathrm{g}$ and of CMK-3 is $1250 \mathrm{~m}^{2} / \mathrm{g}$ [61]. The plot of the pore volume versus the pore diameter (Fig. 3) shows that the pore diameters present in the material are mainly smaller than $100 \mathrm{~nm}$. 


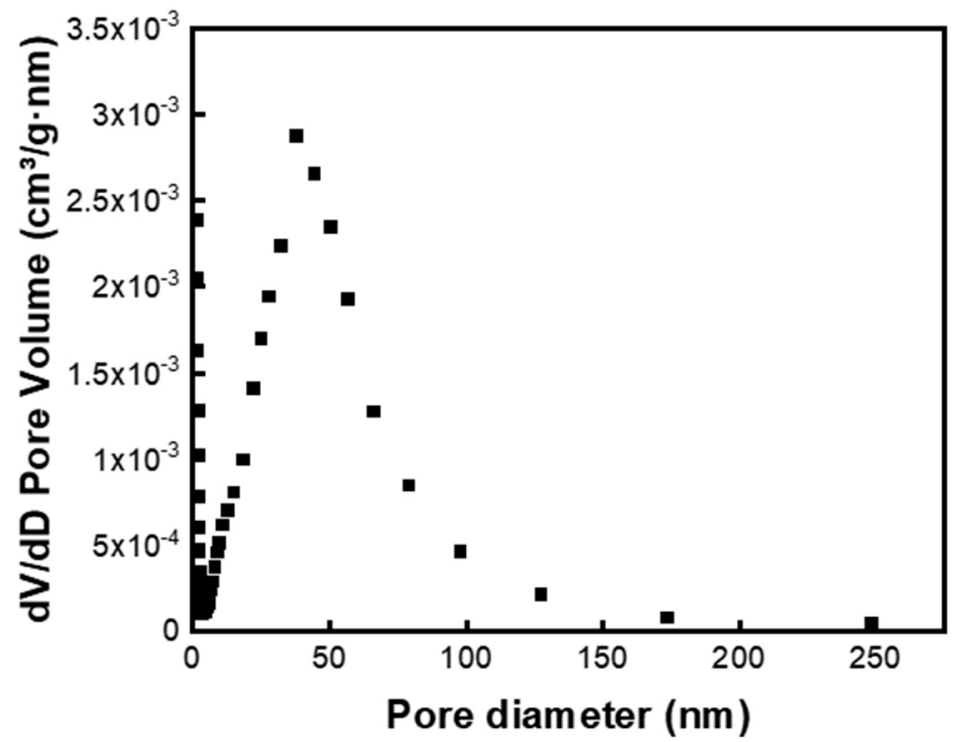

Figure 3. Pore volume-diameter distribution of PS-Nin beads.

\subsection{Membrane morphology}

Figure 4 presents the SEM images of the developed HF MMM. The MMM is composed of blended PES and PVP, which are polymers already widely used for the production of HD membranes. PES has exceptional filtering characteristics, thermal stability, mechanical strength, chemical inertness and it can withstand all sterilization techniques [62]. In order to develop a membrane with high water transport and low fouling properties, we blended PES with PVP, a non-ionic, highly polar, amphiphilic, physiologically inert water-soluble polymer which acts as hydrophilic agent [63].

The cross-section image of the MMM (Fig. 4a) shows corrugated lumen morphology of the membrane. The grooves are well axially aligned with the flow direction and they are not expected to disturb mass transfer, flow rate and transmembrane pressure along the fiber. In addition, their presence in the lumen side of the fiber increases the active membrane surface area and therefore the membrane flux. Also, the rather thick membrane wall allows high particles loading, thus enhancing binding properties of the MMM.

From the magnification of the wall of the MMM (Fig. 4b), a finger-like macrovoids structure, typical of PES-based membranes [64, 65], is visible at the lumen and outer sides of the membrane. These macrovoids vanish along the center of the wall cross-section, where more PS-Nin particles are hosted 
in the polymer matrix. The sorbent particles seem to be quite well dispersed, without aggregation and they are well surrounded by the polymer solution (white arrows in Fig. 4b). Both the lumen and the outer layers of the MMM (Fig. 4c and 4d, respectively) present very thin dense layers with no visible macropores at the lumen surface (Fig. 4e) or at the outer surface (Fig. 4f). However, the outer dense layer (Fig. $4 \mathrm{~d})$ is slightly thicker $(0.5 \mu \mathrm{m})$ compared to the inner dense layer $(0.2 \mu \mathrm{m})$. For this reason, we think that the membrane selective layer, determining the flux and selectivity, is the outer dense layer. We did not observe elution of the particles from the membrane matrix neither during storage in demineralized-water and handling of the MMM HF nor during transport, static and dynamic adsorption experiments (results presented later).

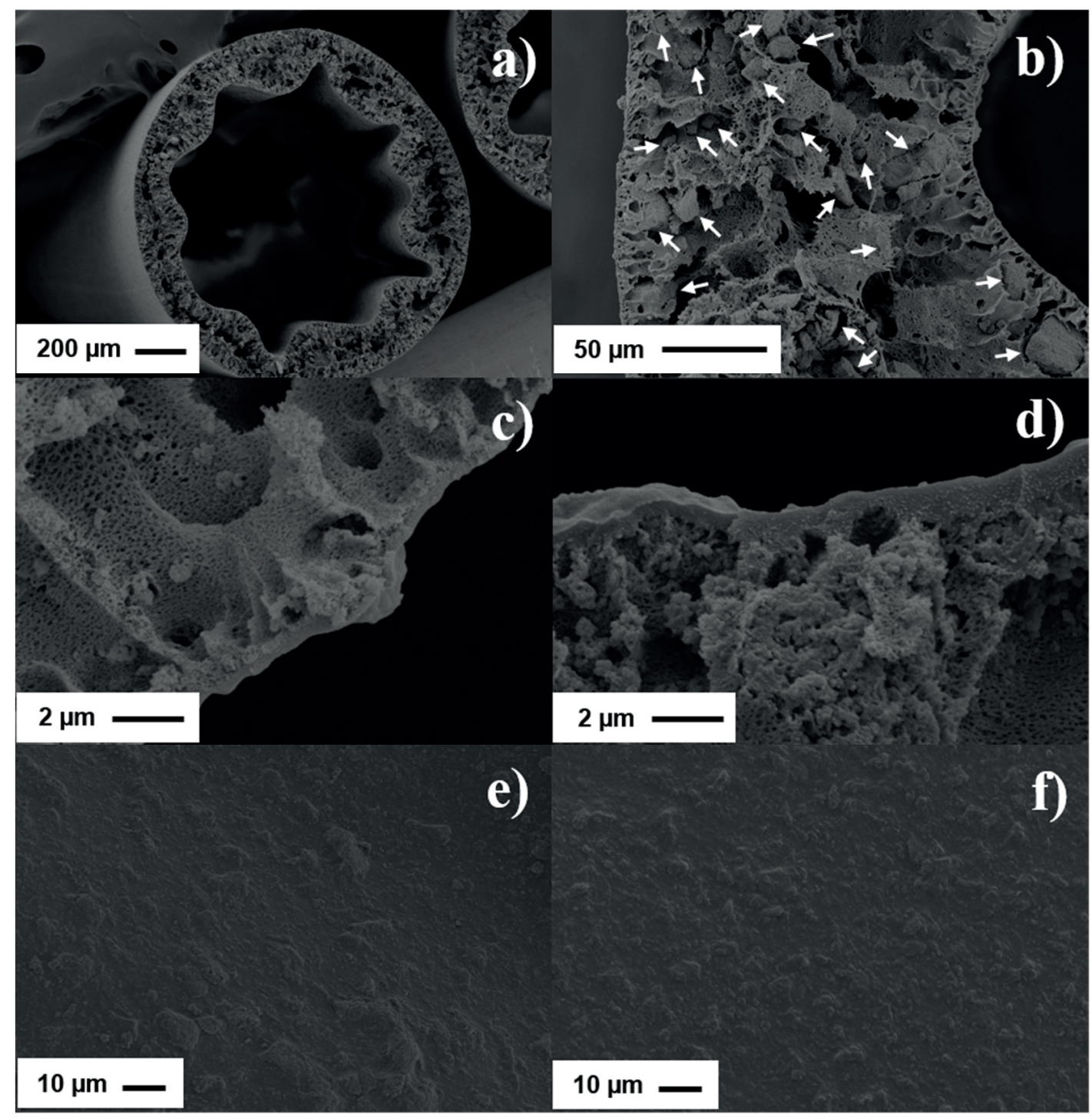

Figure 4. SEM images of the MMM. a) Cross section, b) Magnification of the wall, c) Magnification of the lumen layer, d) Magnification of the outer layer, e) Magnification of the lumen surface, f) Magnification of the outer surface. 


\subsection{Water transport experiments}

Figure 5 presents the graph of the membrane water flux versus TMP. The membrane water permeance, estimated from the slope, is quite high $\left(238 \pm 9 \mathrm{~L} /\left(\mathrm{m}^{2} \cdot \mathrm{h} \cdot\right.\right.$ Bar $\left.)\right)$, typical of a high flux ultrafiltration membrane. The water flux through the membrane increases linearly over pressure, without any indication of membrane compaction. Overall, these results suggest that the MMM has proper morphology characteristics, filtration properties and good mechanical stability to be used for the filtration of urea.

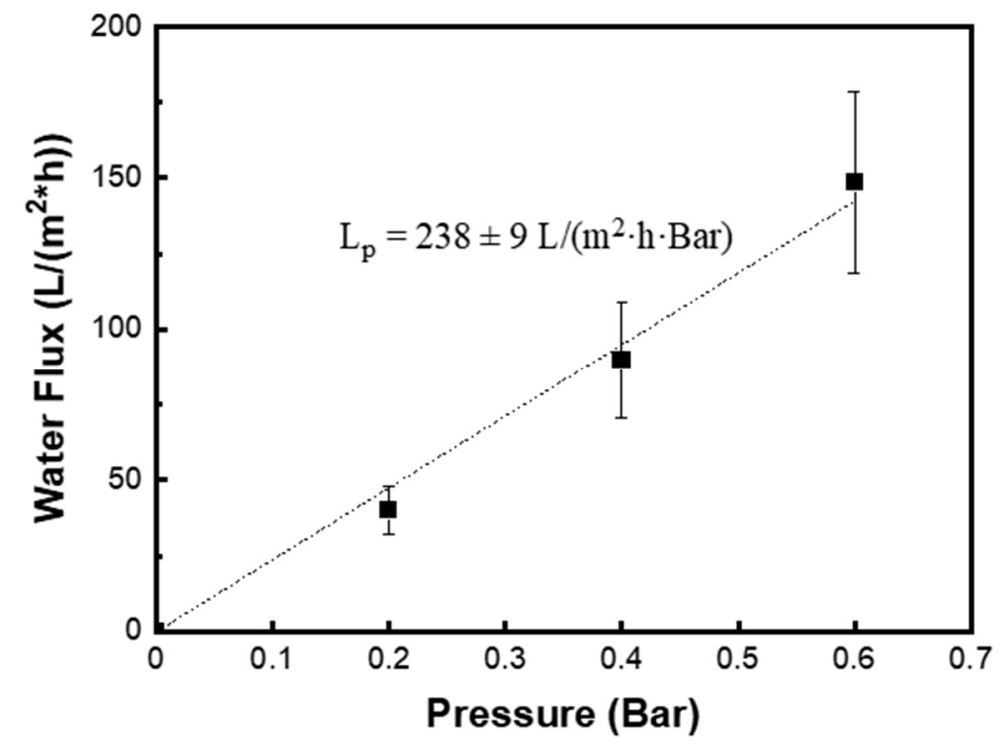

Figure 5. Water permeance of the $\operatorname{MMM}(\mathrm{n}=6$; average \pm standard deviation).

\subsection{Static urea removal studies}

\subsubsection{Effect of temperature on static urea removal by PS-Nin beads $(483 \pm 282 \mu \mathrm{m})$}

Carbonyl-type urea sorbents are characterized by relatively slow urea sorption [10]. The covalent binding of urea is a thermally activated process and the reaction rate increases at higher temperatures. To assess the urea binding kinetics of the PS-Nin beads and the influence of temperature under static conditions, the beads were incubated with a $30 \mathrm{mM}$ urea solution in PBS at 37,50 , and $70{ }^{\circ} \mathrm{C}$ and the urea concentration in the supernatant was monitored for $24 \mathrm{~h}$. Figure 6 shows that, indeed, at higher temperatures the urea binding kinetics is higher, as already observed for similar ninhydrin-type 
sorbent [50]. At $70^{\circ} \mathrm{C}$ the PS-Nin beads remove $1.4 \pm 0.0 \mathrm{mmol} / \mathrm{g}$ of urea over a period of 24 hours, which is significantly higher compared to the removal at $50^{\circ} \mathrm{C}$ and $37^{\circ} \mathrm{C}$ for the same period of time. It also seems that maximum urea binding of approximately $2.7 \mathrm{mmol} / \mathrm{g}$ (estimated considering that the predicted amount of phenyl groups of polystyrene transformed in ninhydrin units is $55 \%$, as reported by Smakman et al. $[48,51])$ is not reached at 24 hours at $70^{\circ} \mathrm{C}$. Possibly longer experiments are required for this.

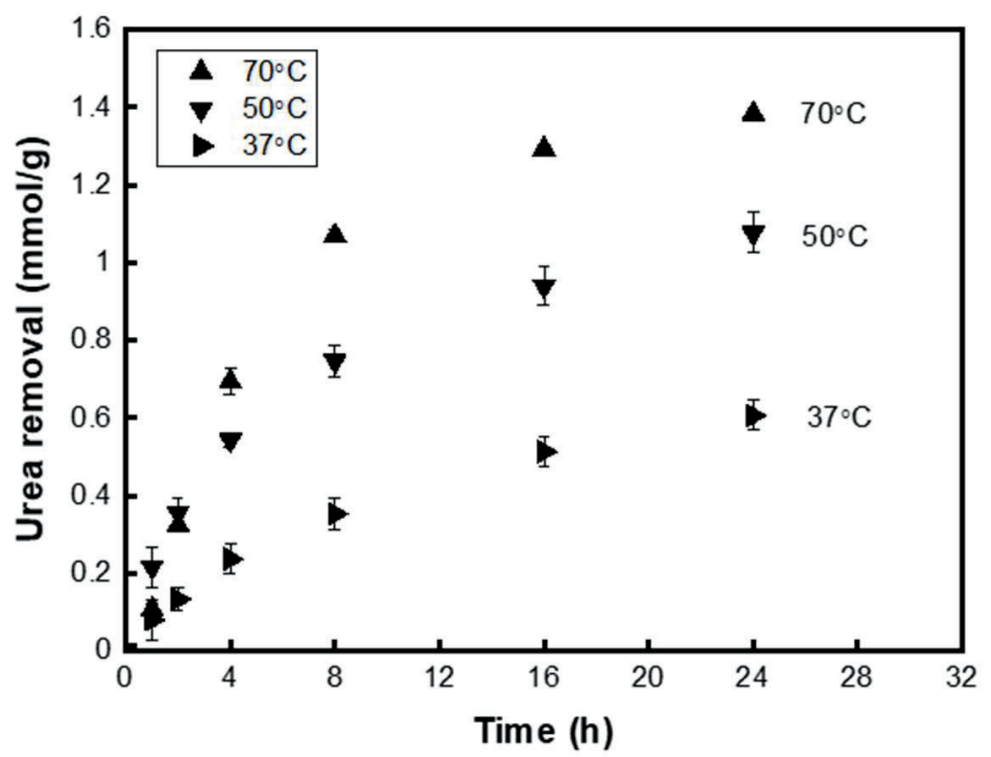

Figure 6. Urea removal of PS-Nin beads $(483 \pm 282 \mu \mathrm{m})$ at different temperatures $(\mathrm{n}=3$; average \pm standard deviation).

\subsubsection{Static urea removal by PS-Nin beads, PS-Nin particles and MMM}

Figure 7a presents the static urea removal at $70{ }^{\circ} \mathrm{C}$ of the PS-Nin beads $(483 \pm 282 \mu \mathrm{m})$ and of the PSNin particles $(<63 \mu \mathrm{m})$. Urea removal is significantly faster for the small particles; for example, at 4 hours urea removal is more than double that by the big PS-Nin beads. Urea removal seems to reach saturation at $1.7 \pm 0.1 \mathrm{mmol} / \mathrm{g}$ for the small PS-Nin particles at approximately 4 hours, whereas for the large PS-Nin beads urea removal reaches $1.4 \pm 0.1 \mathrm{mmol} / \mathrm{g}$ at $24 \mathrm{~h}$. We think that the faster kinetics and higher removal of urea for the smaller PS-Nin particles is due to the increased specific surface area of the particles which allows for the better accessibility of urea molecules to the ninhydrin moieties. Nevertheless, the maximum theoretical binding capacity (approximately $2.7 \mathrm{mmol} / \mathrm{g}$, 
considering that the predicted amount of phenyl groups of polystyrene transformed in ninhydrin units is $55 \%$, as reported by Smakman et al. $[48,51]$ ) was not reached, perhaps due to particle aggregation and poor dispersion of the particles in the suspension, leading to limited accessibility of the ninhydrin moieties to urea. Despite that, our results clearly indicate the advantage of using small particles to achieve faster and higher urea removal.

Figure $7 \mathrm{~b}$ compares the static urea removal of the small PS-Nin particles and of the MMM containing the small PS-Nin particles at $70{ }^{\circ} \mathrm{C}$. For both cases, the urea removal is expressed as mmol of urea removed per grams of sorbent particles. Urea static binding kinetics of the particles in suspension and of the particles in the MMM is comparable over a period of 24 hours (Fig. 7b). Interestingly, at 8 hours urea removal by the particles in suspension seems to reach a plateau, while the removal by the particles embedded in the MMM continues to increase. At 24 hours, urea removal by the particles in suspension is $1.7 \pm 0.1 \mathrm{mmol} / \mathrm{g}$, while the MMM showed binding up to $2.1 \pm 0.1 \mathrm{mmol} / \mathrm{g}$. The higher urea removal in the latter could be due to the better particle dispersion without aggregation within the MMM (SEM image, Fig. 4). Moreover, the polymer matrix does not limit the accessibility of the particles for urea; as a matter of fact, the polymer matrix surrounding the particles is highly porous (Fig. 4) as also indicated by the very high water permeance of the membrane (Fig. 5).

Fig. 7c presents the sorption isotherm of urea on the MMM and on the PES/PVP control HF at 70 ${ }^{\circ} \mathrm{C}$ versus equilibrium urea concentration $(\mathrm{mM})$. Urea binding is expressed as mmol of urea per grams of sorbent particles for the MMM and per grams of membranes for the PES/PVP control HF. The PES/PVP control HF does not remove urea, indicating that the sorbent particles in the MMM are solely responsible for urea binding. Interestingly, at the equilibrium concentration of $90.1 \mathrm{mM}$, urea removal by the MMM corresponds to $3.4 \pm 0.1 \mathrm{mmol} / \mathrm{g}$, which is much higher than the maximum theoretical urea binding (approximately $2.7 \mathrm{mmol} / \mathrm{g}$, considering that the predicted amount of phenyl groups of polystyrene transformed in ninhydrin units is $55 \%,[48,51])$. This exciting result suggests that there is another mechanism playing a role in urea removal by the MMM besides chemisorption of urea to the ninhydrin moieties. This may be based on physisorption, possibly via hydrogen bonding 
between multi-layered urea molecules, similar to what was described by Cheah et al. for mesoporous silica [29]. Possibly, the local urea concentration within the MMM polymer network and around the particles is high leading to enhanced hydrogen bonding between urea molecules and high urea physisorption.
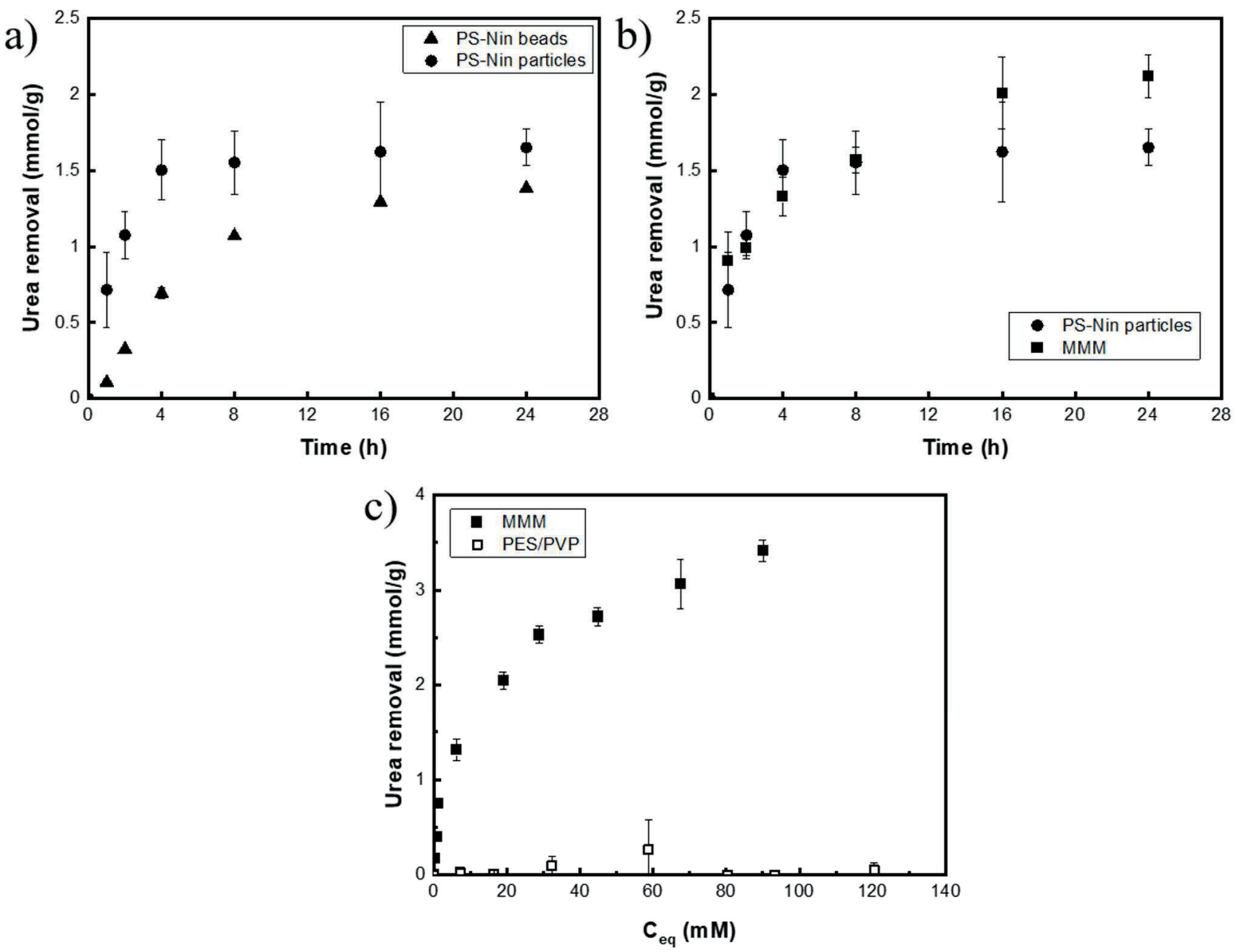

Figure 7. a) Static urea removal of the PS-Nin beads $(483 \pm 282 \mu \mathrm{m})$ and of the PS-Nin particles $(<63 \mu \mathrm{m})$. b) Static urea removal of the PS-Nin particles and of the MMM. c) Urea binding isotherm on the MMM and on the PES/PVP control HF. For a), b) and c) the removal experiments were performed at $70{ }^{\circ} \mathrm{C}$. The data are expressed as average \pm standard deviation $(n=3)$. Urea removal of the MMM is calculated as millimoles of urea removed per grams of particles incorporated in the membrane matrix. Urea removal of the PES/PVP HF is calculated as millimoles of urea removed per grams of membrane. 


\subsection{Dynamic urea removal}

Figure 8 presents the removal of urea by the MMM during filtration and recirculation of a dialysate model solution spiked with urea through the MMM HF at $70{ }^{\circ} \mathrm{C}$ and comparison with the removal of urea by the MMM in static conditions $\left(70{ }^{\circ} \mathrm{C}\right)$. The results show that, over a period of 4 hours, the membrane is able to remove $3.4 \pm 0.3 \mathrm{mmol} / \mathrm{g}$ of urea without reaching saturation (mmol of urea removed per gram of particles incorporated in the MMM). Moreover, the removal kinetics is much higher compared to the static conditions (Fig. 8). As a matter of fact, over a period of 2 hours the MMM is able to remove almost double amount of urea $(2.0 \pm 0.6 \mathrm{mmol} / \mathrm{g})$ than the same MMM in static conditions $(1.0 \pm 0.1 \mathrm{mmol} / \mathrm{g})$ (Fig. 8). Possibly, recirculation and filtration of urea solution through the MMM enhances the particles accessibility to urea.

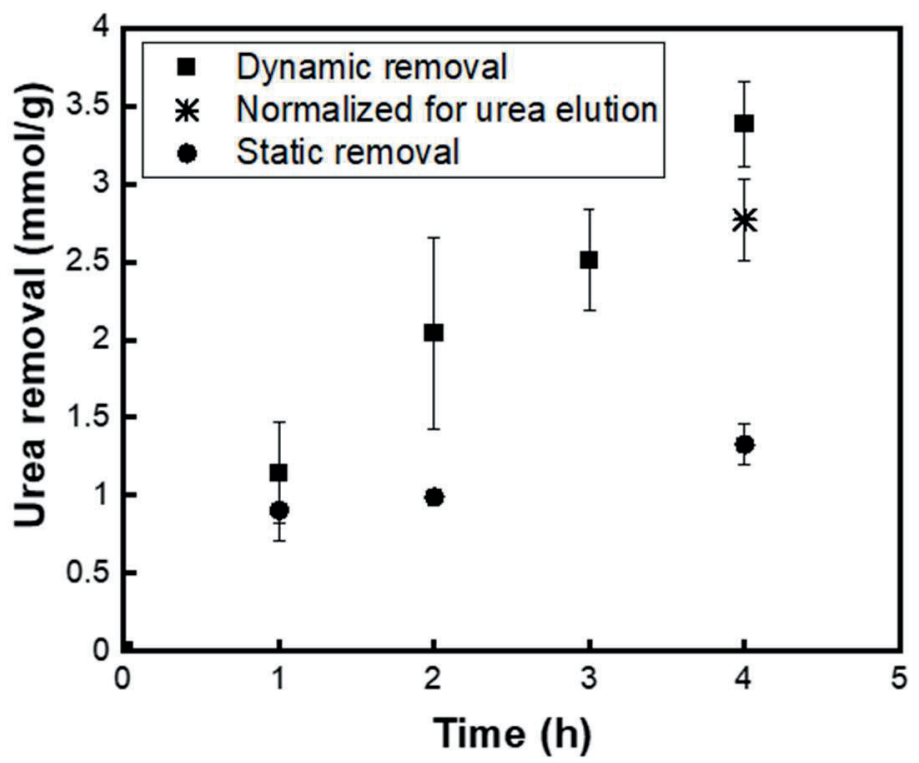

Figure 8. Dynamic removal of urea by the MMM at $70{ }^{\circ} \mathrm{C}(\mathrm{n}=5$, average \pm standard deviation $)$ and comparison with urea removal by the MMM in static conditions at $70^{\circ} \mathrm{C}$. Urea removal by the MMM is calculated as millimoles of urea per grams of particles incorporated in the membrane matrix. * at $4 \mathrm{~h}$ represents the remaining urea in the MMM after eluting loosely bound urea $(n=2$, average \pm standard deviation $)$

Besides higher urea binding kinetics, the total amount of urea removed by the MMM under dynamic conditions is higher compared to the static conditions (Fig. 8). Again, the amount of urea removed per gram of particles in the MMM is higher than the maximum removal of urea expected based on the ninhydrin moieties in the particles (approximately $2.7 \mathrm{mmol} / \mathrm{g}[48,51]$ ). As discussed earlier for 
the binding isotherm, urea molecules are probably adsorbed via hydrogen bonding (physisorption) on urea covalently bound (chemisorption) to the ninhydrin moieties of the particles, thus having multilayered adsorption [29]. When the MMM modules $(n=2)$ were rinsed with ultrapure water at the end of the experiment for 1 hour, 0.05 millimoles of urea were eluted (see Fig. 8) indicating that there the urea removal by the MMM is a combination of chemisorption and physisorption.

Based on the removal in dynamic conditions at $4 \mathrm{~h}$, considering a daily production of urea equal to $400 \mathrm{mmol} /$ day for a typical western diet $[12,13]$ and assuming comparable urea removal when applied in WAK or portable systems, we estimate that 215 grams of MMM would be needed to remove the daily produced urea from spent dialysate at $70^{\circ} \mathrm{C}$. Obviously in the spent dialysate there are other organic compounds (such as creatinine) which could compete with urea for adsorption to the particles and possibly the required MMM weight should be higher in the clinical situation. An important consideration is that the PS-Nin particles, as all the other carbonyl-based sorbents, are not selective towards urea [10] and can also react with other nucleophilic compounds present in the spent dialysate (such as creatinine). However, the amount of membrane estimated here is quite small indicating its good potential for application to PAK/WAK, where miniaturization and lightweight are required. Besides, the competitive adsorption can be avoided by inserting an activated carbon (AC) sorbent column upstream of the urea sorbent MMM module [10].

In comparison to literature, the binding of urea by our MMM $\left(3.4 \pm 0.3 \mathrm{mmol} / \mathrm{g}, 4\right.$ hours, $\left.70{ }^{\circ} \mathrm{C}\right)$ is one of the highest values reported so far (Table 2) [10]. For example, the removal of urea by means of physisorption with $\mathrm{AC}$, graphene oxide, zeolites and MXenes nanosheets is reported for most of the works lower than $1 \mathrm{mmol} / \mathrm{g}[25,31]$. Interestingly, Cheah et al. reported a high removal of urea on mesoporous silica via physisorption (hydrogen bonding) in the order of $8-9 \mathrm{mmol} / \mathrm{g}$ [29] (Table 2). However this work reports an unlikely high urea adsorption capacity for AC used as reference (compared to other studies for AC), thus suggesting that this work might need further validation [10]. Sorbent systems composed of chitosan complexed with metal ions, as copper and zinc, have higher affinity for urea compared to activated carbon, graphene oxide, zeolites and MXenes nanosheets, in 
certain cases reaching urea removal values higher than $4 \mathrm{mmol} / \mathrm{g}[33,35,66,67]$. Nevertheless, the toxicity derived by potential copper leaching is a concern which limits the use of these systems [10]. Finally, other studies implemented oxystarch [41, 43], oxycellulose [42] and cyclodextrin [68] as urea sorbents and reported high urea removal, comparable to the removal with our MMM. However, in contrast to these systems the carbonyl-type sorbents optimally distributed within the MMM can overcome typical issues of sorbents bed column systems, such as aggregation and pressure drop.

Table 2. Selected urea sorbent materials reported in literature $([10,49,50])$ and their urea binding in comparison to the urea binding of the MMM.

\begin{tabular}{|l|c|c|}
\hline \multicolumn{1}{|c|}{ Material } & $\begin{array}{c}\text { Urea binding } \\
\text { reported (mmol/g) }\end{array}$ & Reference \\
\hline PS-Nin particles embedded in PES/PVP MMM & 3.4 & This work \\
\hline Commercial activated carbon (Sigma-Aldrich Co. St. Louis) & 0.4 & {$[25]$} \\
\hline Graphene oxide & 0.5 & {$[25]$} \\
\hline Zeolite ZSM-5 with $\mathrm{SiO}_{2}: \mathrm{Al}_{2} \mathrm{O}_{3}=23: 400$ & 0.7 & {$[25]$} \\
\hline $\mathrm{Ti}_{3} \mathrm{C}_{2} \mathrm{~T}_{\mathrm{x}} \mathrm{MXenes}$ nanosheets & 0.3 & {$[31]$} \\
\hline Silica (SBA-15) & 8.3 & {$[29]$} \\
\hline Silica (Amine functionalized SBA-15) & 9.0 & {$[29]$} \\
\hline Chitosan/Cu ${ }_{2}{ }^{+}$macroporous particles & 2.0 & {$[33]$} \\
\hline Cross-linked Chitosan/Cu${ }_{2}{ }^{+}$copolymer & 4.4 & {$[35,66]$} \\
\hline Cross-linked Chitosan/Zn ${ }_{2}^{+}$particle & 0.8 & {$[67]$} \\
\hline Oxystarch gel & $0.1-0.2$ & {$[41]$} \\
\hline Oxycellulose gel & $0.1-0.2$ & {$[42]$} \\
\hline Oxystarch particles & 3.0 & {$[43]$} \\
\hline Oxidized crosslinked cyclodextrin particles & 1.1 & {$[68]$} \\
\hline Zr/glyoxal complex immobilized on activated carbon or on Zirconium sorbent & $0.2-0.5$ & {$[44]$} \\
\hline Polystyrene Divinylbenzene copolymer - Glyoxaldehyde & 1.5 & {$[47]$} \\
\hline Polystyrene Divinylbenzene copolymer - ninhydrin & $1.1-2.0$ & {$[48]$} \\
\hline Polystyrene - phenylglyoxaldehyde (PGA) beads & $1.4-1.8$ & {$[49]$} \\
\hline Polyvinylphenylethan-1-one - phenylglyoxaldehyde (PGA) beads & $1.8-2.2$ & {$[49]$} \\
\hline Polyvinylindanone - ninhydrin beads & 3.2 & {$[50]$} \\
\hline
\end{tabular}

Since the results of our study were obtained at $70^{\circ} \mathrm{C}$, application of MMM in a WAK or PAK would require heating up of the MMM module. To avoid this, future research would focus on further improving urea removal by the $\mathrm{MMM}$ at $37^{\circ} \mathrm{C}$, for example, by using highly porous sorbent particles 
with higher density of ninhydrin groups. Also, application of high dialysate flow rates and / or higher transmembrane pressure could further improve urea removal.

\section{Conclusions and outlook}

In this work, a new MMM for urea removal from dialysate was developed based on PS-Nin sorbent particles, which can bind urea, embedded in a PES/PVP polymer matrix. Our results show that the incorporation of small sorbent particles in the MMM significantly increases urea removal in comparison to the particles in suspension, thanks to the good dispersion of the sorbents in the MMM without aggregation therewith improving accessibility of urea to the ninhydrin moieties. In dynamic removal experiments, the MMM removes approximately $3.4 \mathrm{mmol} / \mathrm{g}$ of urea after 4 hours of recirculation, which is much higher than the theoretical urea removal expected based on the covalent binding to the ninhydrin moieties on the particles. Elution experiments with water suggest that part of this removal is due to urea physisorption, possibly via urea hydrogen bonding to urea molecules already covalently bound to the sorbent particles (chemisorption). Although our experiments were done using dialysate model solution with only urea, and not any other compounds which could adsorb to the particles, the amount of MMM estimated for the removal of the daily produced urea $(215 \mathrm{~g})$ is quite small suggesting that this MMM can be a promising candidate for application in WAK and PAK systems.

\section{Acknowledgments}

I. Geremia. acknowledges the financial support of Health-Holland TKI project NOVAMEM (project no: LSHM16059-SGF). D. Stamatialis and K. G. F. Gerritsen acknowledge the support by the Strategic Agency of the University of Twente, University of Utrecht and University Medical Center Utrecht and the "European Uremic Toxin working group" (EUTox) of the "European Society for 
Artificial Organs" (ESAO) endorsed by the "European Renal Association-European Dialysis Transplantation Association" (ERA-EDTA), of which D. Stamatialis is a member. J. A. W. Jong, C. F. van Nostrum, W. Hennink and K. G. F. Gerritsen acknowledge the Dutch organization for Scientific Research NWO-TTW (project no: 14433) and the Dutch Kidney Foundation. 


\section{References}

[1] C. Zoccali, U. Moissl, C. Chazot, F. Mallamaci, G. Tripepi, O. Arkossy, P. Wabel, S. Stuard, Chronic fluid overload and mortality in ESRD, Journal of the American Society of Nephrology 28(8) (2017) 2491-2497.

[2] W.R. Clark, N.L. Dehghani, V. Narsimhan, C. Ronco, Uremic toxins and their relation to dialysis efficacy, Blood Purification 48(4) (2019) 299-314.

[3] J. Himmelfarb, B. Ratner, Wearable artificial kidney: problems, progress and prospects, Nature Reviews Nephrology 16 (2020) 558-559.

[4] W.H. Fissell, S. Roy, A. Davenport, Achieving more frequent and longer dialysis for the majority: wearable dialysis and implantable artificial kidney devices, Kidney International 84(2) (2013) 256-264.

[5] M.K. van Gelder, S.M. Mihaila, J. Jansen, M. Wester, M.C. Verhaar, J.A. Joles, D. Stamatialis, R. Masereeuw, K.G.F. Gerritsen, From portable dialysis to a bioengineered kidney, Expert Review of Medical Devices 15(5) (2018) 323-336.

[6] P. Armignacco, A. Lorenzin, M. Neri, F. Nalesso, F. Garzotto, C. Ronco, Wearable devices for blood purification: principles, miniaturization, and technical challenges, Seminars in Dialysis 28(2) (2015) 125-130.

[7] P. Armignacco, F. Garzotto, M. Neri, A. Lorenzin, C. Ronco, Wak engineering evolution, Blood Purification 39(1-3) (2015) 110-114.

[8] J.W.M. Agar, Green dialysis: the environmental challenges ahead, Seminars in Dialysis 28(2) (2015) 186192.

[9] C. Boccato, D.W. Evans, R. Lucena, J. Vienken, Water and Dialysis Fluids: A Quality Management Guide, Pabst Science Publishers, 2015.

[10] M.K. van Gelder, J.A.W. Jong, L. Folkertsma, Y. Guo, C. Blüchel, M.C. Verhaar, M. Odijk, C.F. Van Nostrum, W.E. Hennink, K.G.F. Gerritsen, Urea removal strategies for dialysate regeneration in a wearable artificial kidney, Biomaterials 234 (2020) 119735.

[11] J.W.M. Agar, Review: Understanding sorbent dialysis systems, Nephrology 15(4) (2010) 406-411.

[12] I.D. Weiner, W.E. Mitch, J.M. Sands, Urea and ammonia metabolism and the control of renal nitrogen excretion, Clinical Journal of the American Society of Nephrology 10(8) (2015) 1444-1458. 
[13] C.S. Shinaberger, R.D. Kilpatrick, D.L. Regidor, C.J. McAllister, S. Greenland, J.D. Kopple, K. KalantarZadeh, Longitudinal associations between dietary protein intake and survival in hemodialysis patients, American Journal of Kidney Diseases 48(1) (2006) 37-49.

[14] R. Vanholder, T. Gryp, G. Glorieux, Urea and chronic kidney disease: the comeback of the century? (in uraemia research), Nephrology Dialysis Transplantation 33(1) (2017) 4-12.

[15] H.D. Lehmann, R. Marten, C.A. Gullberg, How to catch urea? Considerations on urea removal from hemofiltrate, Artificial Organs 5(3) (1981) 278-285.

[16] B. Krajewska, Ureases I. Functional, catalytic and kinetic properties: a review, Journal of Molecular Catalysis B: Enzymatic 59(1) (2009) 9-21.

[17] B. Krajewska, Ureases. II. Properties and their customizing by enzyme immobilizations: a review, Journal of Molecular Catalysis B: Enzymatic 59(1) (2009) 22-40.

[18] M. Roberts, The regenerative dialysis (REDY) sorbent system, Nephrology 4(4) (1998) 275-278.

[19] C. Mion, B. Branger, R. Issautier, H.A. Ellis, M. Rodier, S. Shaldon, Dialysis fracturing osteomalacia without hyperparathyroidism in patients treated with HCO3 rinsed REDY cartridge, American Society for Artificial Internal Organans Journal 27(1) (1981).

[20] S.R. Ash, Sorbents in treatment of uremia: a short history and a great future, Seminars in Dialysis 22(6) (2009) 615-622.

[21] M. Wester, F. Simonis, N. Lachkar, W.K. Wodzig, F.J. Meuwissen, J.P. Kooman, W.H. Boer, J.A. Joles, K.G. Gerritsen, Removal of urea in a wearable dialysis device: a reappraisal of electro-oxidation, Artificial Organs 38(12) (2014) 998-1006.

[22] J.C. Wright, A.S. Michaels, A.J. Appleby, Electrooxidation of urea at the ruthenium titanium oxide electrode, American Institute of Chemical Engineers Journal 32(9) (1986) 1450-1458.

[23] B. Schuenemann, E. Quellhorst, H. Kaiser, G. Richter, K. Mundt, E. Weidlich, G. Loeffler, M. Zachariae, O. Schunk, Regeneration of filtrate and dialysis fluid by electro-oxidation and absorption, Transactions American Society for Artificial Internal Organs 28 (1982) 49-53.

[24] M. Wester, M.K. van Gelder, J.A. Joles, F. Simonis, D.H.M. Hazenbrink, T.W.M. van Berkel, K.R.D. Vaessen, W.H. Boer, M.C. Verhaar, K.G.F. Gerritsen, Removal of urea by electro-oxidation in a miniature dialysis device: a study in awake goats, American Journal of Physiology. Renal Physiology 315(5) (2018) 1385-1397. 
[25] Y.C. Cheng, C.C. Fu, Y.S. Hsiao, C.C. Chien, R.S. Juang, Clearance of low molecular-weight uremic toxins $\mathrm{p}$-cresol, creatinine, and urea from simulated serum by adsorption, Journal of Molecular Liquids 252 (2017).

[26] C.H. Ooi, W.K. Cheah, Y.L. Sim, S.Y. Pung, F.Y. Yeoh, Conversion and characterization of activated carbon fiber derived from palm empty fruit bunch waste and its kinetic study on urea adsorption, Journal of Environmental Management 197 (2017) 199-205.

[27] J.H. Kim, J.C. Kim, J.H. Moon, J.Y. Park, K.K. Lee, E. Kang, H.C. Kim, B.G. Min, C. Ronco, Development of a Cold Dialysate Regeneration System for Home Hemodialysis, Blood Purification 28(2) (2009) 84-92.

[28] C. Giordano, R. Esposito, P. Bello, E. Quarto, F.M. Gonzalez, Cold carbon apparatus for hemodialysis, Journal of Dialysis 1(2) (1976) 165-179.

[29] W.K. Cheah, Y.L. Sim, F.Y. Yeoh, Amine-functionalized mesoporous silica for urea adsorption, Materials Chemistry and Physics 175 (2016) 151-157.

[30] V. Wernert, O. Schäf, H. Ghobarkar, R. Denoyel, Adsorption properties of zeolites for artificial kidney applications, Microporous and Mesoporous Materials 83(1) (2005) 101-113.

[31] F. Meng, M. Seredych, C. Chen, V. Gura, S. Mikhalovsky, S. Sandeman, G. Ingavle, T. Ozulumba, L. Miao, B. Anasori, Y. Gogotsi, MXene sorbents for removal of urea from dialysate: a step toward the wearable artificial kidney, ACS Nano 12(10) (2018) 10518-10528.

[32] J. Liu, X. Chen, Z. Shao, P. Zhou, Preparation and characterization of chitosan/Cu(II) affinity membrane for urea adsorption, Journal of Applied Polymer Science 90(4) (2003) 1108-1112.

[33] Y.G. Zhou, Y.D. Yang, X.M. Guo, G.R. Chen, Effect of molecular weight and degree of deacetylation of chitosan on urea adsorption properties of copper chitosan, Journal of Applied Polymer Science 89(6) (2003) $1520-1523$.

[34] A. Pathak, S.K. Bajpai, Preparation of Cu(II)-Immobilized Chitosan (CIC) and preliminary urea uptake study, Polymer-Plastics Technology and Engineering 47(9) (2008) 925-930.

[35] L.D. Wilson, C. Xue, Macromolecular sorbent materials for urea capture, Journal of Applied Polymer Science 128(1) (2013) 667-675.

[36] T. Alizadeh, M. Akhoundian, Z. Movahed, Indirectly determination of urea using chloride ion selective electrode after its extraction and preconcentration by molecularly imprinted polymer, Analytical and Bioanalytical Electrochemistry 1(4) (2009) 246 - 256. 
[37] T. Alizadeh, Preparation of molecularly imprinted polymer containing selective cavities for urea molecule and its application for urea extraction, Analytica Chimica Acta 669(1-2) (2010) 94-101.

[38] B. Khadro, C. Sanglar, A. Bonhomme, A. Errachid, N. Jaffrezic-Renault, Molecularly imprinted polymers (MIP) based electrochemical sensor for detection of urea and creatinine, Procedia Engineering 5 (2010) 371374.

[39] Y. Cheng, K. Xu, H. Li, Y. Li, B. Liang, Preparation of Urea-Imprinted Cross-Linked Chitosan and Its Adsorption Behavior, Analytical Letters 47(6) (2014) 1063-1078.

[40] J.A.W. Jong, R. Smakman, M.E. Moret, M.C. Verhaar, W.E. Hennink, K.G.F. Gerritsen, C.F. Van Nostrum, Reactivity of (vicinal) carbonyl compounds with urea, ACS Omega 4(7) (2019) 11928-11937.

[41] T. Shimizu, S. Fujishige, A newly prepared surface-treated oxystarch for removal of urea, Journal of Biomedical Materials Research 17(4) (1983) 597-612.

[42] D. Deepak, Evaluation of adsorbents for the removal of metabolic wastes from blood, Medical and Biological Engineering and Computing 19(6) (1981) 701-706.

[43] M.N.Z. Abidin, P.S. Goh, A.F. Ismail, N. Said, M.H.D. Othman, H. Hasbullah, M.S. Abdullah, B.C. Ng, S.H.S.A. Kadir, F. Kamal, Highly adsorptive oxidized starch nanoparticles for efficient urea removal, Carbohydrate Polymers 201 (2018) 257-263.

[44] R.J. Wong, Materials for removal of toxins in sorbent dialysis and methods and systems using same, WO2013101888A1, 2014.

[45] E. Kuntz, J.P. Quentin, Process for extracting urea from a solution with alkenylaromatic polymers with a-ketoalhydic groups, US4012317A, 1974.

[46] E. Kuntz, J.P. Quentin, Alkenylaromatics polymers with a-ketoalhydic groups, US3933753A, 1976.

[47] M.J. Poss, H. Blom, A. Odufu, R. Smakman, Macromolecular ketoaldehydes, US 2009/0218290A1, 2009.

[48] R. Smakman, Macromolecular carbonyl groups containing material suitable for use as sorbent for nitrogen compounds, US4897200A, 1990.

[49] J.A.W. Jong, Y. Guo, C. Veenhoven, M.E. Moret, J. van der Zwan, A. Lucini Paioni, M. Baldus, K.C. Scheiner, R. Dalebout, M.J. van Steenbergen, M.C. Verhaar, R. Smakman, W.E. Hennink, K.G.F. Gerritsen, C.F. van Nostrum, Phenylglyoxaldehyde-functionalized polymeric sorbents for urea removal from aqueous solutions, ACS Applied Polymer Materials 2(2) (2020) 515-527. 
[50] J.A.W. Jong, Y. Guo, D. Hazenbrink, S. Douka, D. Verdijk, J. van der Zwan, K. Houben, M. Baldus, K.C. Scheiner, R. Dalebout, M.C. Verhaar, R. Smakman, W.E. Hennink, K.G.F. Gerritsen, C.F. van Nostrum, A ninhydrin-type urea sorbent for the development of a wearable artificial kidney, Macromolecular Bioscience 20(3) (2020) 1900396.

[51] R. Smakman, A.W. van Doorn, Urea removal by means of direct binding, Clinical Nephrology 26 (Suppl.1) (1986) 58-62.

[52] R. Shapiro, N. Chatterjie, Cyclization reactions of ninhydrin with aromatic amines and ureas, The Journal of Organic Chemistry 35(2) (1970) 447-450.

[53] D. Pavlenko, E. van Geffen, M.J. van Steenbergen, G. Glorieux, R. Vanholder, K.G.F. Gerritsen, D. Stamatialis, New low-flux mixed matrix membranes that offer superior removal of protein-bound toxins from human plasma, Scientific Reports 6(1) (2016) 34429.

[54] M.S. Tijink, M. Wester, G. Glorieux, K.G. Gerritsen, J. Sun, P.C. Swart, Z. Borneman, M. Wessling, R. Vanholder, J.A. Joles, D. Stamatialis, Mixed matrix hollow fiber membranes for removal of protein-bound toxins from human plasma, Biomaterials 34(32) (2013) 7819-7828.

[55] I. Geremia, R. Bansal, D. Stamatialis, In vitro assessment of mixed matrix hemodialysis membrane for achieving endotoxin-free dialysate combined with high removal of uremic toxins from human plasma, Acta Biomaterialia 90 (2019) 100-111.

[56] C.C.L. Schuurmans, A. Abbadessa, M.A. Bengtson, G. Pletikapic, H.B. Eral, G. Koenderink, R. Masereeuw, W.E. Hennink, T. Vermonden, Complex coacervation-based loading and tunable release of a cationic protein from monodisperse glycosaminoglycan microgels, Soft Matter 14(30) (2018) 6327-6341.

[57] M. Thommes, K. Kaneko, A.V. Neimark, J.P. Olivier, F. Rodriguez-Reinoso, J. Rouquerol, K.S.W. Sing, Physisorption of gases, with special reference to the evaluation of surface area and pore size distribution (IUPAC Technical Report), Pure and Applied Chemistry 87(9-10) (2015) 1051-1069.

[58] J.A.W. Jong, M.-E. Moret, M.C. Verhaar, W.E. Hennink, K.G.F. Gerritsen, C.F. van Nostrum, Effect of substituents on the reactivity of ninhydrin with urea, Chemistry Select 3(4) (2018) 1224-1229.

[59] T. Uma Devi, N. Lawrence, R. Ramesh Babu, S. Selvanayagam, H. Stoeckli-Evans, K. Ramamurthi, Characterization of a newly synthesized organic nonlinear optical crystal: urea ninhydrin monohydrate, Journal of Crystal Growth 311(13) (2009) 3485-3490.

[60] M. Manivannan, S. Rajendran, Investigation of inhibitive action of urea- Zn 2+ system in the corrosion control of carbon steel in sea water, International Journal of Engineering Science and Technology 3(11) (2011) 8048-8060. 
[61] D. Pavlenko, D. Giasafaki, G. Charalambopoulou, E. van Geffen, K.G.F. Gerritsen, T. Steriotis, D. Stamatialis, Carbon adsorbents with dual porosity for efficient removal of uremic toxins and cytokines from human plasma, Scientific Reports 7(1) (2017) 14914.

[62] M. Kohlova, C.G. Amorim, A. Araujo, A. Santos-Silva, P. Solich, M. Montenegro, The biocompatibility and bioactivity of hemodialysis membranes: their impact in end-stage renal disease, Journal of Artificial Organs 22(1) (2019) 14-28.

[63] M. Irfan, A. Idris, Overview of PES biocompatible/hemodialysis membranes: PES-blood interactions and modification techniques, Materials Science and Engineering: C 56 (2015) 574-592.

[64] Y. Liu, G.H. Koops, H. Strathmann, Characterization of morphology controlled polyethersulfone hollow fiber membranes by the addition of polyethylene glycol to the dope and bore liquid solution, Journal of Membrane Science 223(1) (2003) 187-199.

[65] Z.-L. Xu, F. Alsalhy Qusay, Polyethersulfone (PES) hollow fiber ultrafiltration membranes prepared by PES/non-solvent/NMP solution, Journal of Membrane Science 233(1) (2004) 101-111.

[66] C. Xue, L.D. Wilson, Kinetic study on urea uptake with chitosan based sorbent materials, Carbohydrate Polymers 135 (2016) 180-186.

[67] X. Liu, S. Sun, Y. Tang, S. Li, J. Chang, L.A. Guo, Y. Zhao, Preparation and kinetic modeling of crosslinked chitosan microspheres immobilized Zn(II) for urea adsorption, Analytical Letters 45(12) (2012) 16321644.

[68] H. Bing-Lin, X.-B. Zhao, Study of the oxidation of crosslinked $\beta$-cyclodextrin polymer and its use in the removal of urea. I, Reactive Polymers 18(3) (1992) 229-235. 



\title{
Chapter 6
}

\section{Development of positively charged nanofiltration hollow fiber}

\section{membranes for ammonium removal}

\author{
I. Geremia, M. Damhuis, D. Stamatialis
}

(Bio)artificial organs, Department of Biomaterials Science and Technology, TechMed Centre, Faculty of Science and Technology, University of Twente, P.O. Box 217, 7500 AE Enschede, The Netherlands 


\begin{abstract}
When kidneys lose their functionality, patients need to undergo hemodialysis (HD). For standard HD of 4 hours, $280-500 \mathrm{~L}$ of water is used to generate $120 \mathrm{~L}$ of pure dialysate. The continuous regeneration of spent dialysate could lead to a significant reduction of water consumption in HD and is an important requirement for wearable artificial kidneys (WAK) development. The most difficult substance to remove from spent dialysate is urea, as it is small, water soluble and unreactive. Several methods have been proposed for this but they have not found clinical implementation due to their inefficiency and/or because lead to production of undesirable toxic byproducts.
\end{abstract}

One method for removing urea is its hydrolysis via urease and the consequent removal of the produced ammonium ions. Here, we investigate the development of positively charged nanofiltration hollow fibers (PCN) for the retention of ammonium ions. To fabricate the PCN fibers, we implement the 'Chemistry in the Spinneret' technique, using polyetherimide and polyethyleneimine as membrane forming material and positively charged crosslinker, respectively. Various spinning parameters are tailored, including the bore solution composition and flow rate as well as the polymer dope concentration. The new membrane can be applied to transmembrane pressure up to 6 Bar, it has rather low water permeance of $0.03 \mathrm{~L} /\left(\mathrm{m}^{2} \cdot \mathrm{h} \cdot \mathrm{Bar}\right)$ while its retention of ammonium ions is $50 \%$ after 30 minutes of filtration. 


\section{Introduction}

End Stage Kidney Disease (ESKD) patients have to undergo hemodialysis (HD) if not suitable for or while waiting for kidney transplantation. HD is a life-sustaining therapy; however, it heavily affects patients' social life. As a matter of fact, HD is mainly performed in the clinics 3 times per week for 4 hours, thus requiring a strict compliance of the patient. Also, the intermittence of dialysis treatment poses a problem, as normally the kidneys filter continuously and the body is adjusted to this $[1,2]$. This means that there are periods in which the waste and toxins build up in the blood before being removed, strongly impacting the patients' health $[1,2]$. Moreover, a very large amount of water is used for HD treatment; for standard HD of 4 hours, $280-500 \mathrm{~L}$ of water is used to generate $120 \mathrm{~L}$ of pure dialysate $[3,4]$. Portable $(\mathrm{PAK})$ and wearable artificial kidney (WAK) devices could provide continuous or semi-continuous blood purification and could ameliorate patients' quality of life. Moreover, these systems, for which a small volume of spent dialysate (preferably $0.5 \mathrm{~L}$ or lower) would be continuously regenerated and recirculated, would significantly lower the water consumption of the treatment [5-9]. Currently, the biggest challenge for the regeneration of the spent dialysate is the removal of urea [10], which is the main waste product of nitrogen metabolism [11], due to its small size, high water solubility and neutral charge. Many strategies have been proposed for its removal, including the application of the REDY (Recirculation of DialYsis) device with enzymatic decomposition of urea by mean of ureases [12], the electrooxidation of urea [13-16], which converts urea in nitrogen, carbon dioxide and hydrogen gases, as well as the application of several sorbent systems. The latter can remove urea via (i) physisorption to activated carbon (AC) [17-20], silica [21], zeolites [22] and MXenes nanosheets [23], (ii) via coordination bond, as chitosan-copper complexes, or (iii) covalent binding (chemisorption) such aldehydes, $\alpha-$ ketoaldehyde hydrates, ninhydrins, $\alpha$-ketoesters and glyoxaldehydes [24-29]. Unfortunately, some of the above strategies were withdrawn from the market due to production of toxic 
byproducts and others have not found, yet, clinical implementation due to their low urea removal [10]. Specifically, the urea sorbents had been mainly applied in bed column systems $[25-27,30$, 31], where high pressure drop, rather bad particle dispersion and aggregation limited the amount of available binding sites, thus reducing their urea removal capacity. Application of membranes for urea removal can be a cheaper alternative and could be applicable for longer period of time. Positively charged nanofiltration (PCN) membranes, which are currently mostly applied in water softening, desalination, dyes and heavy metals removal, could be suitable for the removal of ammonium ions derived from the hydrolysis of urea by means of urease, via Donnan exclusion and size retention effects. Nevertheless, most of the membrane forming polymers have negative charge at neutral $\mathrm{pH}[32,33]$ and, therefore, positively-charged membranes are commonly prepared using multi-step procedures, as layer-by-layer deposition $[34,35]$ or thin-film composite (TFC) method $[36,37]$. These multi-step procedures are time consuming, require many deposition's and washing's steps and produce a lot of waste material. Of particular interest for the fabrication of positively charged membranes is the modification of polyetherimide (PEI) membrane through crosslinking with amines, such as polyethyleneimine (pei). The crosslinking reaction there requires only two steps, i.e. membrane fabrication and post-treatment [38-40]. Vanherck et al. [41] reported a method for the crosslinking of several polyimides flat sheet membranes with a diamine or polyamine requiring no additional post-treatment. Kopec et al. [42] proposed the so-called "Chemistry in the spinneret" method for the crosslinking of polyimide hollow fibers with primary amine crosslinkers. There, a crosslinked P84 hollow fiber membrane was fabricated in a single step by dissolving the crosslinker, pei, in the bore solution. The crosslinking occurred while the fiber was formed, thus the hollow fiber membrane was fabricated in one step.

In this work we implement the "Chemistry in the spinneret" for the fabrication of PCN hollow fiber membranes for the potential removal of ammonium ions from the dialysate. These ions can 
be generated by the hydrolysis of urea by means of urease. The new PCN fibers are synthesized via crosslinking of PEI with the positively charged pei, see Figure 1 . The primary amine binds to the nitrogen atom of the imide. The reaction causes the opening of the imide ring and the formation of two amide groups.<smiles>[R]NC(=O)c1ccc([R])cc1C(=O)N[R]</smiles>

Figure 1. Reaction scheme between the imide group of PEI and a primary amine.

Various spinning parameters are tailored to obtain membranes with high ammonium ions retention. The morphology of the membranes is studied via Scanning Electron Microscopy (SEM) and their chemistry and surface charge are investigated by means of infrared spectroscopy, zeta potential and gel content measurements. The membranes' transport properties are accessed via clean water and ammonium ions transport experiments. 


\section{Materials and Methods}

\subsection{Positively-charged nanofiltration (PCN) hollow fibers fabrication}

For the preparation of the polymer dope, an appropriate amount of polyetherimide (PEI, Ultem 1000, molecular weight $(\mathrm{MW})=55000 \mathrm{~g} / \mathrm{mol}$, kindly provided by Sabic, Riyadh, Saudi Arabia) was weighed and added to N-methylpyrrolidone (NMP, analytical grade, Sigma-Aldrich Chemie $\mathrm{GmbH}$, Munchen, Germany) (Table 1). The mixture was allowed to dissolve at $70{ }^{\circ} \mathrm{C}$ under gentle stirring. The polymer solution was then filtered using a custom-made pressurized setup with a metal mesh STAL315 $\mu \mathrm{m}$ filter (Bekaert S.A., Kortrijk, Belgium) and transferred in a stainlesssteel syringe. The syringe was left overnight to degas. For the preparation of the bore solution, $10 \mathrm{wt} \%$ of polyethyleneimine (pei, MW = $25000 \mathrm{~g} / \mathrm{mol}$, Sigma-Aldrich Chemie GmbH, Munchen, Germany) was added to ultrapure water or to a mixture of NMP and ultra-pure water (see details on Table 1). The solution was then left on a roller-bank until fully mixed. The bore solution was then transferred into a plastic syringe and left overnight to degas.

The stainless-steel syringe containing the dope solution and the plastic syringe containing the bore solution were connected to high-pressure syringe pumps and to a spinneret for the preparation of the fibers. Two different spinnerets were used, depending on the concentration of PEI in the dope solution (Table 1 and 2). The dope solution was extruded at $1 \mathrm{~mL} / \mathrm{min}$ whereas the bore solution was extruded at various flow rates (Table 1). After exiting the spinneret, the nascent fiber travelled through a $10 \mathrm{~cm}$ airgap before entering a demineralized water coagulation bath under the influence of gravity (no take-up wheel was employed). After fabrication, the hollow fibers were rinsed several times with fresh demineralized water to remove any residual traces of NMP, before being stored in demineralized water. Table 1 summarises the spinning conditions for the various fibers prepared and Table 2 reports the dimensions of the spinnerets used for the hollow fiber fabrication. 
Table 1. Spinning conditions for the fabrication of the PCN hollow fibers

\begin{tabular}{|c|c|c|c|c|c|}
\hline Fiber & $\begin{array}{c}\text { Dope solution } \\
\text { (PEI/NMP) } \\
\text { (wt\%) }\end{array}$ & $\begin{array}{c}\text { Bore solution } \\
\text { (pei/NMP/water) } \\
\mathbf{( w t / )}\end{array}$ & $\begin{array}{c}\text { Dope flow rate } \\
\mathbf{( m L / m i n )}\end{array}$ & $\begin{array}{c}\text { Bore flow rate } \\
\text { (mL/min) }\end{array}$ & Spinneret \\
\hline F1 & $20 / 80$ & $10 / 0 / 90$ & 1 & 1 & $\mathrm{~S}$ \\
\hline F2 & $20 / 80$ & $10 / 0 / 90$ & 1 & 2 & $\mathrm{~S}$ \\
\hline F3 & $20 / 80$ & $10 / 0 / 90$ & 1 & 1 & $\mathrm{~S}$ \\
\hline F4 & $20 / 80$ & $10 / 20 / 70$ & 1 & 1 & $\mathrm{~S}$ \\
\hline F5 & $20 / 80$ & $10 / 45 / 45$ & 1 & 1 & $\mathrm{~S}$ \\
\hline F6 & $20 / 80$ & $10 / 70 / 20$ & 1 & 1 & $\mathrm{~S}$ \\
\hline F7 & $25 / 75$ & $10 / 0 / 90$ & 1 & 1 & $\mathrm{~B}$ \\
\hline F8 & $28 / 72$ & $10 / 0 / 90$ & & & \\
\hline
\end{tabular}

Table 2. Dimensions of the two spinnerets used for the fabrication of the PCN hollow fibers

\begin{tabular}{|l|c|c|}
\hline & Spinneret S & Spinneret B \\
\hline Needle Diameter $(\mathbf{m m})$ & 0.6 & 1.35 \\
\hline Dope Inner Diameter $\mathbf{( m m )}$ & 1.2 & 1.65 \\
\hline Dope Outer Diameter $(\mathbf{m m})$ & 1.4 & 2.4 \\
\hline
\end{tabular}

\subsection{Module Preparation}

The spun hollow fibers were cut into pieces of approximately $50 \mathrm{~cm}$. They were then placed in a 25 wt\% glycerol (Sigma-Aldrich Chemie GmbH, Munchen, Germany) solution and left on a horizontal shaker overnight. The fibers were dried overnight on tissue papers. Modules were made by placing 4 fiber pieces of approximately $25 \mathrm{~cm}$ in polyethylene tubing and gluing both ends with a 2-compontent epoxy glue (Griffon Combi Snel-Rapide, Bison International, Goes, The Netherlands). The fibers spun with Spinneret S had an average lumen surface area per module of around $16 \mathrm{~cm}^{2}$, while the fibers spun with Spinneret B had an average lumen surface area per module of around $34 \mathrm{~cm}^{2}$. 


\subsection{Scanning Electron Microscopy (SEM)}

Samples for SEM imaging were prepared from pieces of the spun fibers that have been treated with glycerol, dried in air and fractured using liquid nitrogen. For the inner and outer surface images, pieces of fiber were cut with a scalpel at a shallow angle. The samples were then sputtercoated with either gold, using a Cressington 108Auto (Cressington Scientific Instruments, Watford, UK), or platinum, using a JEOL JFC-1300 (JEOL, Tokyo, Japan). SEM images were made using JEOL JSM-6010LA (JEOL, Tokyo, Japan), with an accelerating voltage of $5 \mathrm{kV}$.

\subsection{Attenuated Total Reflection Fourier Transform Infrared Spectroscopy (ATR-FTIR)}

ATR-FTIR was performed on fiber samples not treated with glycerol. The samples were prepared by cutting small pieces of fiber axially and folding them open. The samples were measured using a PerkinElmer Spectrum Two (Waltham, Massachusetts, United States). The samples were scanned four times with a resolution of $4 \mathrm{~cm}^{-1}$ and the scans were averaged. For each fiber at least six samples $(n=6)$ were measured on both the inside surface and outside surface. The Perkin-Elmer Spectrum 10 control software was used to collect the spectra. After collection, the spectra were subjected to automatic baseline and ATR correction.

\subsection{Zeta Potential}

The zeta potential of the inner surface of the PCN hollow fibers $(n=2)$ was determined using a SurPASS Electrokinetic Analyzer (Anton Paar, Graz, Austria), controlled with the VisioLab software. The measurement was performed using $5 \mathrm{mM}$ potassium chloride (Sigma-Aldrich Chemie $\mathrm{GmbH}$, Munchen, Germany) as electrolyte solution, in streaming potential mode and the $\mathrm{pH}$ ranged from 5.5 to 10 . The $\mathrm{pH}$ was adjusted with $1 \mathrm{M}$ nitric acid (Sigma-Aldrich Chemie $\mathrm{GmbH}$, Munchen, Germany) solution and $1 \mathrm{M}$ sodium hydroxide (Sigma-Aldrich Chemie GmbH, Munchen, Germany) solution. 


\subsection{Gel Content}

The gel content of the fibers was determined by dissolving samples not treated with glycerol in NMP over 48 hours, after recording their weight. The NMP was then discarded and the fiber samples were rinsed thoroughly multiple times with ultrapure water. The samples were then dried in an oven at $37^{\circ} \mathrm{C}$ until no more change in weight was observed. Equation 1 was used to estimate the gel content from the initial and final mass $(m)$ of the fibers. The data are presented as average \pm standard deviation $(\mathrm{n}=4)$.

$G C=\frac{m_{\text {final }}}{m_{\text {initial }}} \times 100 \%$

Equation 1

\subsection{Water transport experiments}

Before the water transport experiments, the fiber modules were pre-compacted with ultrapure water at the transmembrane pressure (TMP) of 0.6 Bar for 30 minutes for the F1-5, TMP of 3 Bar for 2 hours for F7 and TMP of 6 Bar for 2 hours for F8. Afterwards, the amount of permeated water was measured over time at the TMP of 0.2, 0.4 and 0.6 Bar for F1-5 (30 minutes at each TMP), at TMP of 1, 2 and 3 Bar for F7 (1 hour at each TMP) and at TMP of 1, 2, 3, 6 Bar for F8 (1h at each TMP). The resulting water permeance $\left(\mathrm{L} /\left(\mathrm{m}^{2} \cdot \mathrm{h} \cdot\right.\right.$ Bar $\left.)\right)$ was calculated as the slope of the linear fit of the flux versus the TMP (Bar). A least 3 modules per fiber type $(n=3)$ were measured; the results are presented as average \pm standard deviations.

\subsection{Ammonium Acetate Retention}

The retention of ammonium acetate by the produced fibers was measured using a solution of 40 mM ammonium acetate (Sigma-Aldrich Chemie GmbH, Munchen, Germany) in ultrapure water. The retention was measured at 3 Bar in dead-end filtration configuration. The module was 
pressurized for 90 minutes and the permeate was collected every 30 minutes. The retentate was collected every period as well, using the tap at the end of the module. The membrane flux was also monitored throughout the experiment. The concentration of ammonium ions in the samples was measured using the Urea CT FS kit (Diasys, Diasys, Holzheim, Germany) with a Cary300 UV-Vis spectrophotometer (Agilent Technologies, Santa Clara, California, United States). The absorption was measured at $688 \mathrm{~nm}$. A modified protocol was used. Briefly, $5 \mu \mathrm{L}$ of $4 \mathrm{x}$ diluted sample was incubated with $500 \mu \mathrm{L}$ of reagent $\mathrm{R} 1$ for 10 minutes at room temperature (RT). A measurement was then performed to get the baseline. Then, $500 \mu \mathrm{L}$ of reagent $\mathrm{R} 2$ was added and the mixture was again incubated at RT for 10 minutes, before reading the absorbance of the sample. Every sample was measured in duplicate and the duplicates were averaged. The measured absorbances were converted to concentration using a calibration curve. The retention was calculated using Equation 2. In this equation, $C$ represents the concentration. The data are presented as average \pm standard deviation ( $\mathrm{n}=2$ for $\mathrm{F} 7, \mathrm{n}=4$ for $\mathrm{F} 8$ ).

Retention $=\left(1-\frac{c_{\text {permeate }}}{c_{\text {retentate }}}\right) \times 100 \%$ Equation 2 


\section{Results and discussion}

\subsection{Morphology and crosslinking of the hollow fiber membranes}

\subsubsection{SEM imaging}

Figure 2 shows the SEM images of the hollow fibers spun using different bore flow rates of 1 $\mathrm{mL} / \mathrm{min}$ (Fiber 1, F1), $2 \mathrm{~mL} / \mathrm{min}$ (Fiber 2, F2) and $3 \mathrm{~mL} / \mathrm{min}$ (Fiber 3, F3). All these fibers display a similar morphology with finger-like macrovoids originating from both the lumen (inside) and outer surface and separated by a spongy layer.

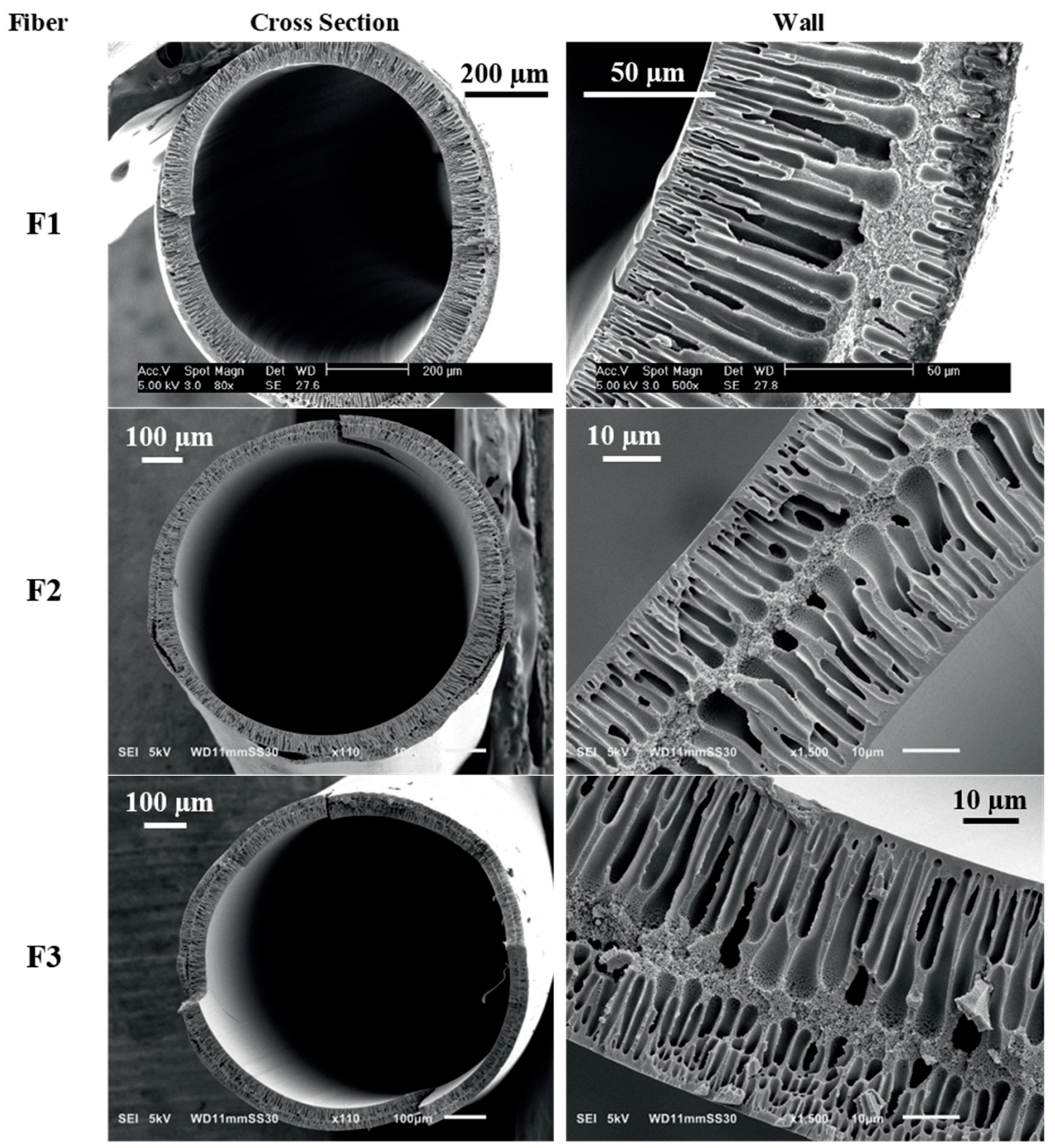

Figure 2. SEM images of fibers F1, F2 and F3. Left: cross section; Right: magnification of the wall 
The wall thickness of the fibers decreases with increasing of the bore flow rate, consistent to the literature $[43,44]$, due to the pressure of the bore solution after exiting the spinneret. On one hand, the presence of macrovoids is an indication that the solvent/non-solvent exchange is very fast $[45,46]$ due to the fact that both the bore solution and the coagulation bath consist of water. This also explains the formation of the dense layers on the inner and outer surface of the fibers. On the other hand, the exchange seems to be not fast enough to allow complete fiber crosslinking in the airgap $(10 \mathrm{~cm})$. This is why the two layers of macrovoids are present. Before full exchange has happened, the fibers enter the coagulation bath, where another rapid exchange of water and NMP starts in the outer part. 
Fiber
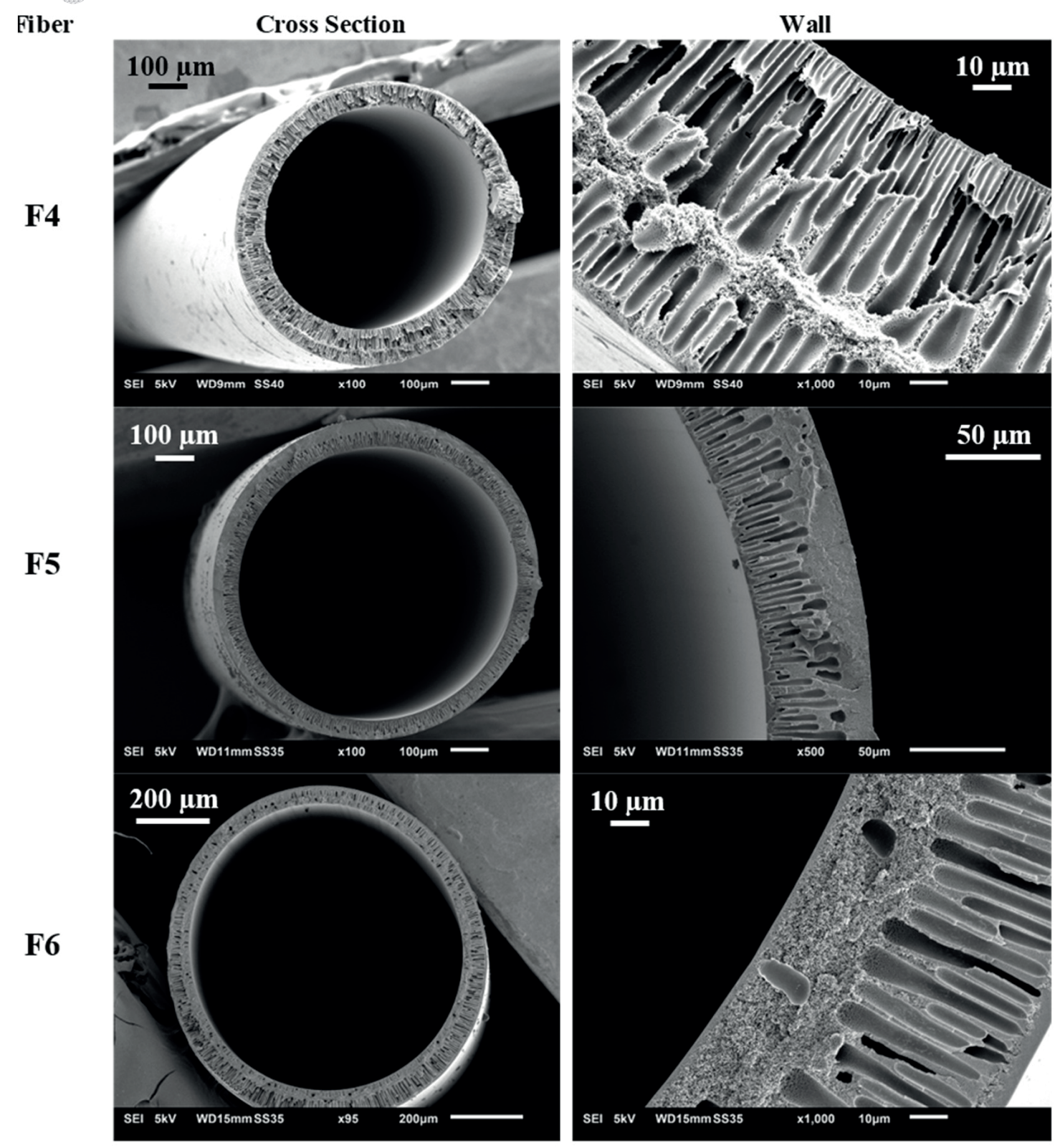

Figure 3. SEM images of fibers F4, F5 and F6. Left: cross section; Right: magnification of the wall.

Figure 3 shows the cross-section of the fibers F4, F5 and F6 spun with different concentrations of NMP in the bore, being $20 \mathrm{wt} \%, 45 \mathrm{wt} \%$ and $70 \mathrm{wt} \%$ of the polymer dope composition, respectively. F4 has similar morphology to the fibers spun with only water as bore liquid, having finger-like macrovoids growing from both sides of the wall towards the middle. F5 has only one layer of finger-like voids, growing from the lumen side towards the middle, while the outer part of the wall is spongy. F6 has the spongy and macrovoids layer in a reverse order compared to F5, with the spongy layer also having some voids. All these fibers have dense layers on both surfaces. It has to be mentioned that the spinning of F6 was difficult. The nascent fiber was 
unstable, fragile and, more importantly, it did not go straight to the bottom of the coagulation bath under the freefall condition used. This could be prevented by using a take-up wheel.

The outer surfaces of the three fibers are porous (Figure 4). The lumen surfaces of F4 and F5 have no visible pores, while F6 has pores on the lumen surface (Figure 4). As expected, the pore size on the lumen layer increases with the increase of the solvent concentration in the bore solution, which dilutes the dope solution at the lumen surface. The selective layer, which determines the performance and selectivity of the membranes, is present at the lumen side for all the three fibers.

F7 and F8 were spun with higher PEI concentration in the dope solution compared to fibers F1F6 (25 wt $\%$ for F7 and $28 \mathrm{wt} \%$ for F8), with dope and bore flow rate of $1 \mathrm{~mL} / \mathrm{min}$ and without NMP in the bore solution. Moreover, F7 and F8 were spun with a larger spinneret (Spinneret B) compared to F1-F6 (Spinneret S) because, due to the high dope concentration and simultaneous crosslinking, the spinneret $\mathrm{S}$ was blocked very often during the spinning of F7 and F8. We also tried to spin fibers with a concentration of $20 \mathrm{wt} \%$ PEI in the dope with the larger spinneret at the chosen flow rate of $1 \mathrm{~mL} / \mathrm{min}$. However, at these spinning conditions the nascent fiber broke in the airgap. 


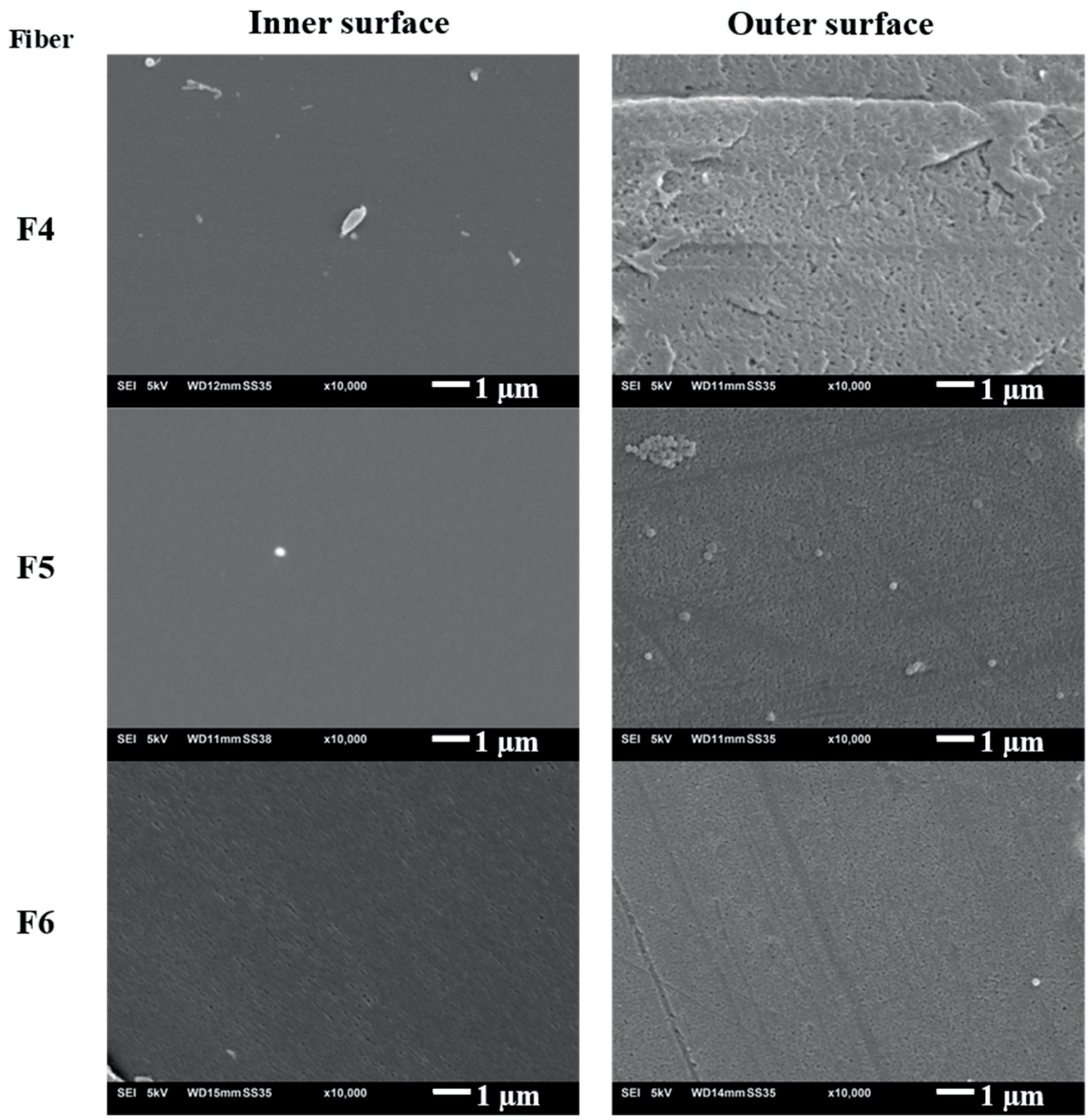

Figure 4. SEM images of the inner (left column) and outer (right column) surfaces of the fibers F4, F5 and F6.

Figure 5 shows that both F7 and F8 have an inner layer with finger-like macrovoids. F7 has a spongy layer in the outer part of the wall, while F8 presents parts of the cross-section with fingerlike macrovoids both in the lumen and outer side and other parts of the cross-section with spongy layer on the outer side of the wall. Figure 6 shows that F7 and F8 have quite porous outer surfaces while the lumen surfaces seem to be denser, suggesting that the selective layer for both fibers is present at the lumen side. The lumen surface of F7 appears smooth, while for F8 it appears to be rougher. This could be due to the higher crosslinking of PEI there 
Fiber

F7

F8
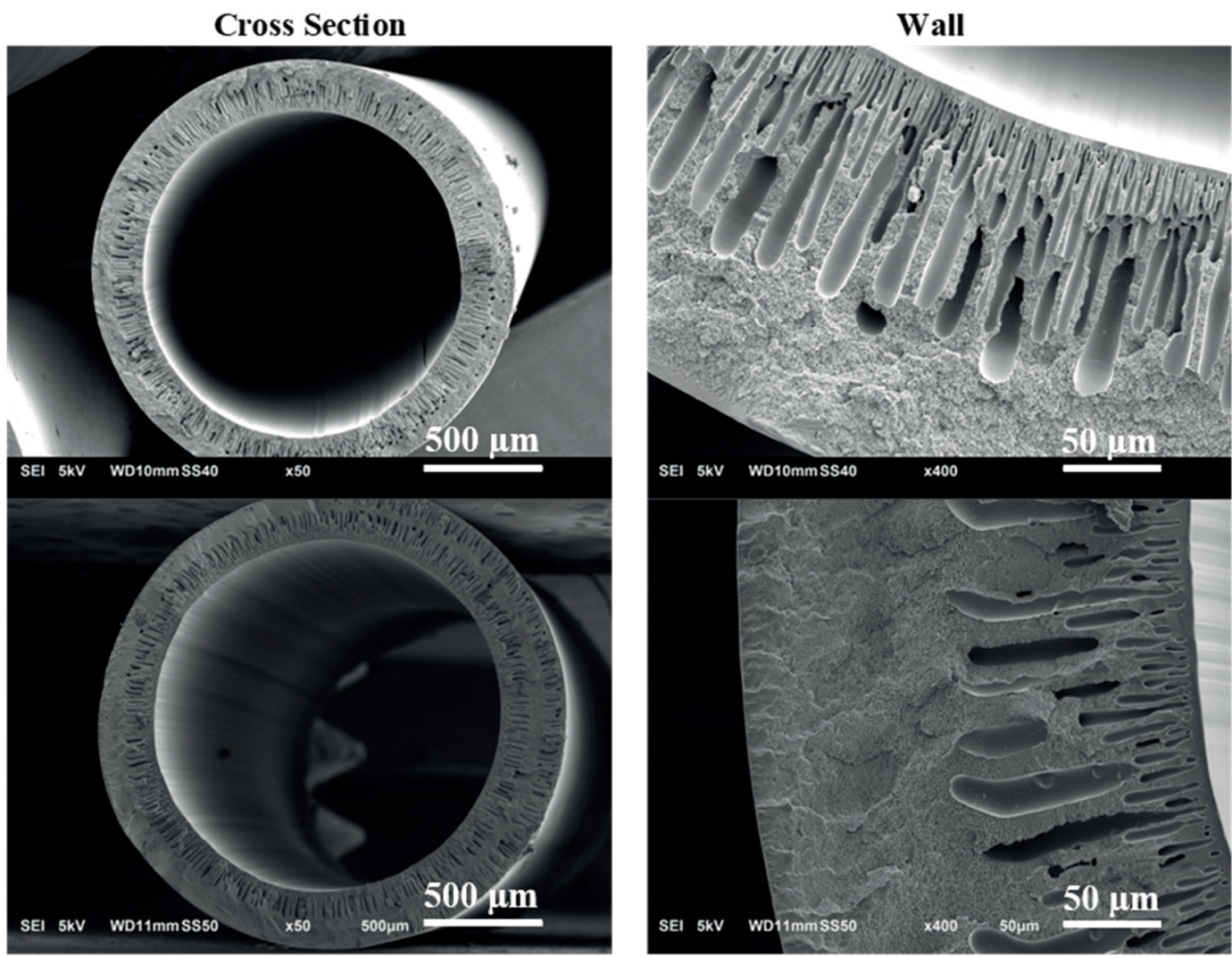

Figure 5. SEM images of fibers F7 and F8. Left: cross section; Right: magnification of the wall.
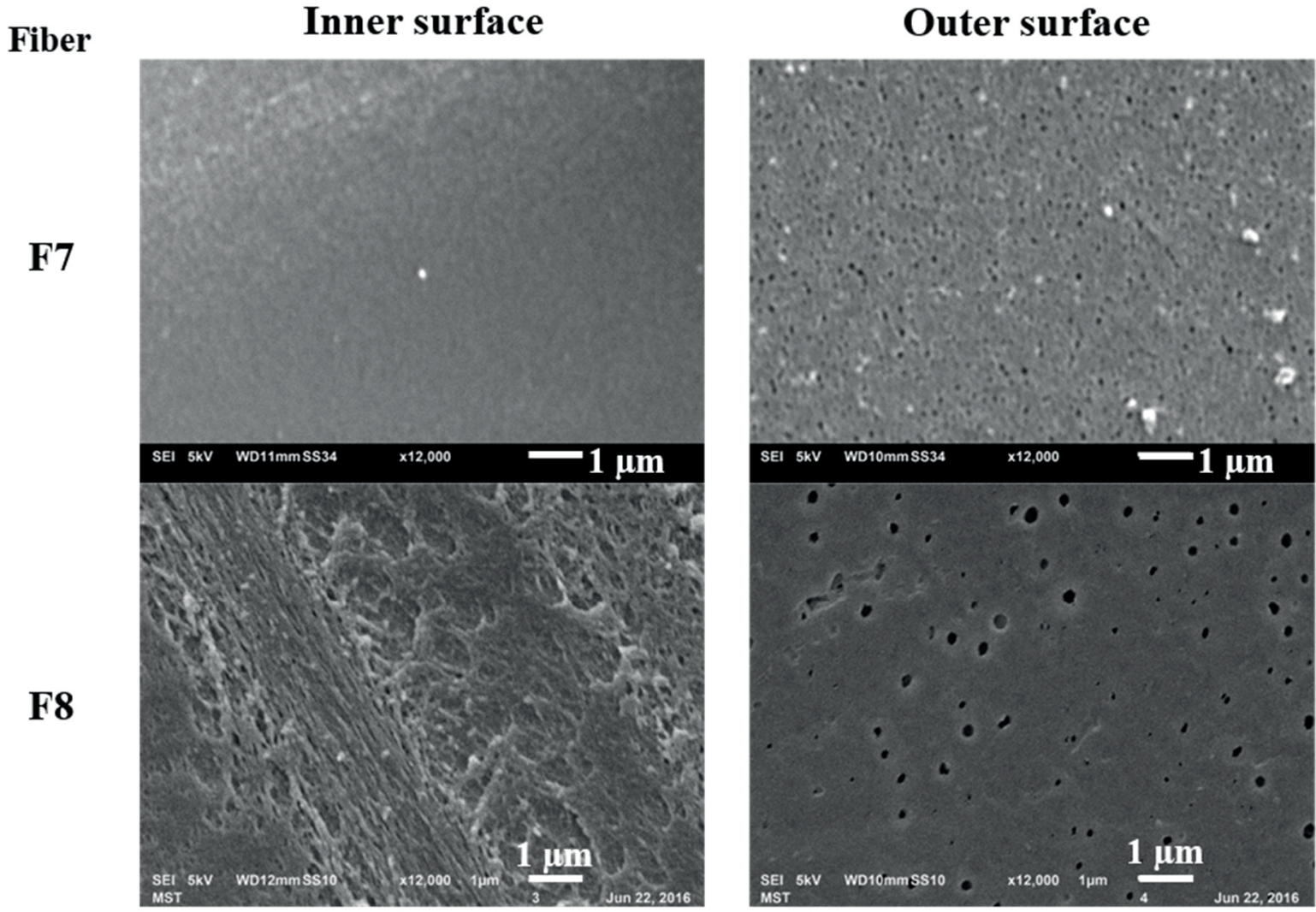

Figure 6. SEM images of the inner (left column) and outer (right column) surfaces of the F7 and F8. 


\subsubsection{Attenuated Total Reflection Fourier Transform Infrared Spectroscopy (ATR-FTIR)}

ATR-FTIR scans of both the lumen and outer surfaces were made for all fibers. The resulting spectra are shown in Figure 7, with the lumen surface in the left column and the outer surface in the right column.

In Figure 7, Box 1 between 3500 and $3000 \mathrm{~cm}^{-1}$ indicates the area where the primary and secondary amines show absorption peaks. Box 2 between 1700 and $1625 \mathrm{~cm}^{-1}$ highlights the absorption of the $\mathrm{C}=\mathrm{O}$ bond of amides and Box 3 between $1600 \mathrm{~cm}^{-1}$ and $1500 \mathrm{~cm}^{-1}$ highlights the absorption of the C-N bond of amides. The influence of the bore flow rate, the amount of solvent in the bore solution and the concentration of PEI in the dope solution on crosslinking with pei were studied and compared to non-crosslinked PEI.

Figures $7 \mathrm{a}$ and $7 \mathrm{~b}$ show the spectra of the lumen and outer surface of the fibers spun with bore flow rates of $1 \mathrm{~mL} / \mathrm{min}(\mathrm{F} 1), 2 \mathrm{~mL} / \mathrm{min}(\mathrm{F} 2)$ and $3 \mathrm{~mL} / \mathrm{min}(\mathrm{F} 3)$ and indicate that crosslinking occurs on both surfaces for all the three fibers, when comparing the spectra to the neat PEI spectrum. The peak intensity is related to the amount of crosslinking, as more functional groups result in a higher absorption. Comparison of the lumen and outer surface spectra suggests that F1 has the least difference in peak intensity between both surface spectra. F2 and F3 have bigger differences in peak intensities between their lumen and outer surfaces, with more crosslinking at the lumen surface. This suggests that when using low bore flow rate of $1 \mathrm{~mL} / \mathrm{min}$, pei permeates the fiber wall much more than when applying higher bore flow rates of $2 \mathrm{~mL} / \mathrm{min}$ or $3 \mathrm{~mL} / \mathrm{min}$. Perhaps there is a maximum value of bore flow rate at which the crosslinker travels through the wall of the nascent fiber and this might be due to the faster phase separation expected at higher bore flow rates. 
a)

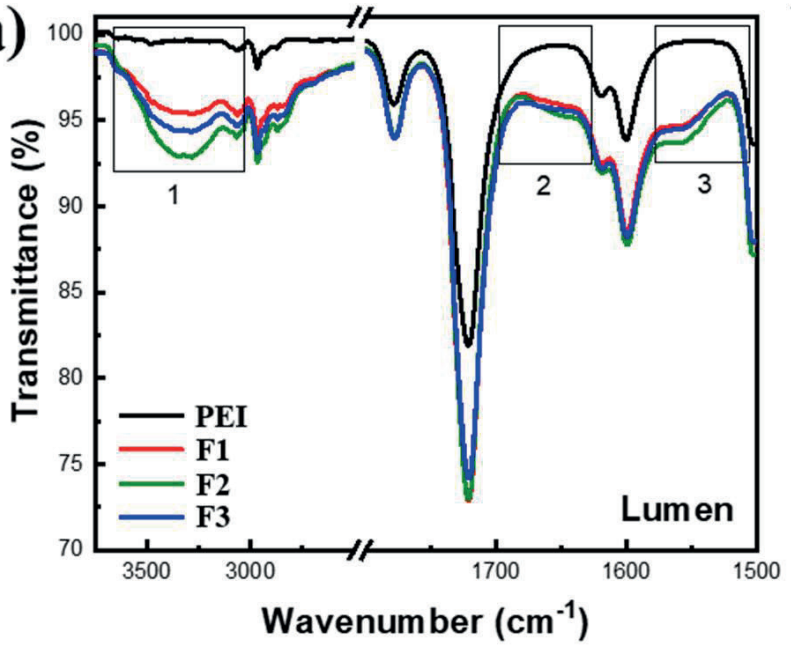

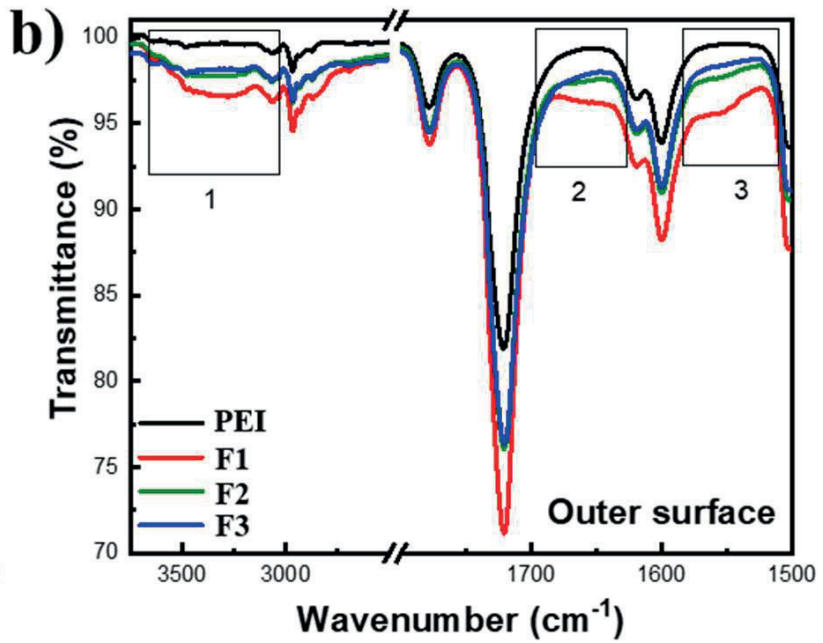

c)

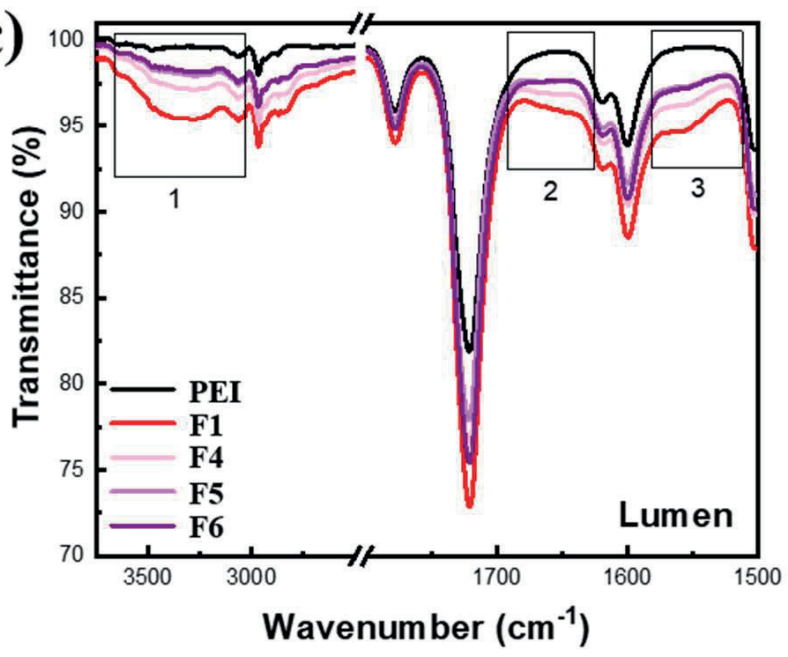

e)

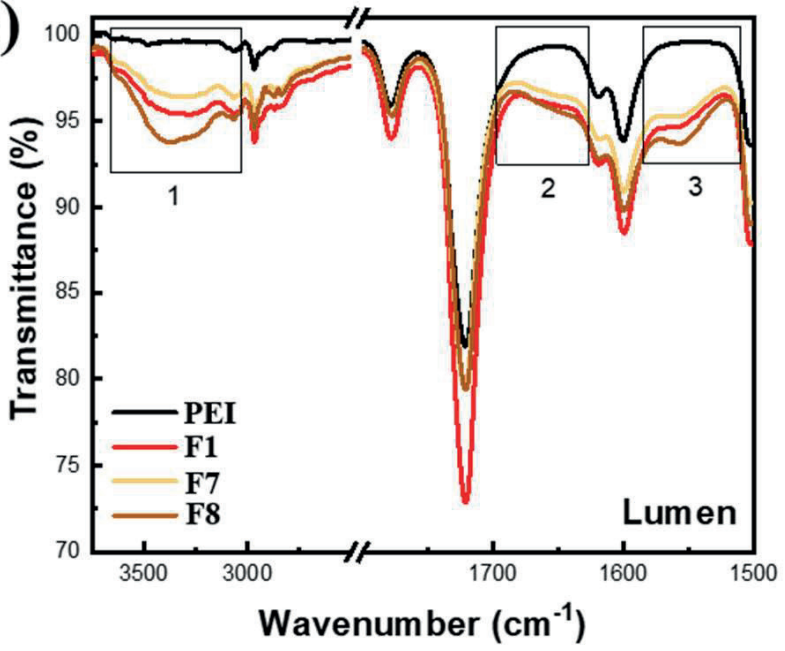

d)
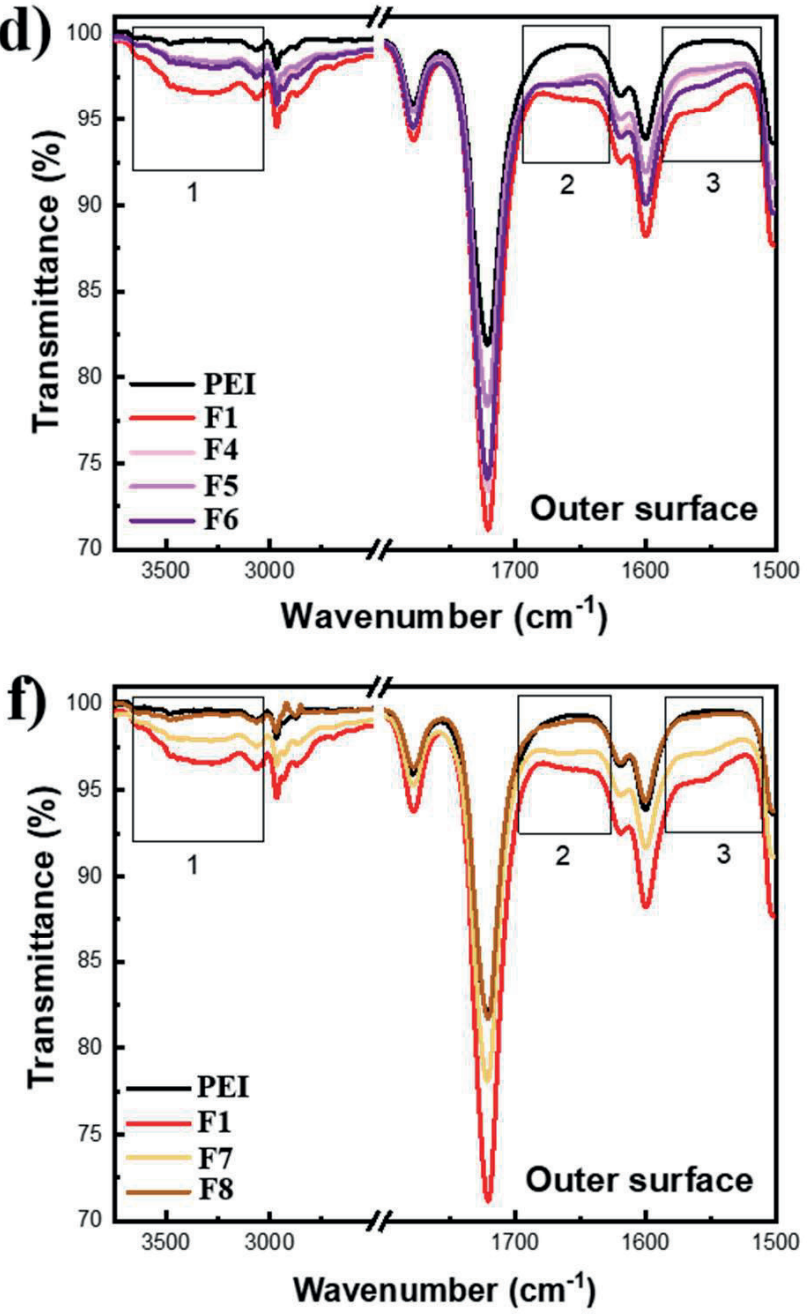

Figure 7: FTIR spectra for all the fibers. Lumen: spectra a), c) and e). Outer surface: spectra b), d) and f). All graphs contain a spectrum of neat PEI, which is a non-crosslinked sample. Box 1 in the graphs indicates the area of primary and secondary amines, while Box 2 and Box 3 in each graph indicate the amide peaks.

Figures $7 \mathrm{c}$ and $7 \mathrm{~d}$ show the ATR-FTIR spectra of the fibers spun with NMP solvent in the bore solution at $25 \mathrm{wt} \%$ (F4), $50 \mathrm{wt} \%$ (F5) and $75 \mathrm{wt} \%$ (F6). For comparison, $\mathrm{F} 1$ is included, as this fiber can be considered to be spun with the same conditions as the other three, with $0 \mathrm{wt} \% \mathrm{NMP}$ 
(only water) in the bore solution. There, the amine and amide peaks have little difference between the lumen and outer surfaces for all three fibers. Comparing the spectra of the lumen surfaces, it can be seen that the absorption in Boxes 1,2 and 3 decreases with increasing amounts of solvent in the bore and that there is no difference between F5 and F6. With regards to the outer surface, F1 has the highest peak in the three highlighted areas than the other three. There seems to be a relation between the amount of solvent in the bore solution and fiber crosslinking. As the amount of solvent in the bore solution increases, the peak intensity decreases on the lumen surface, indicating less crosslinking. The same holds for the fiber outer surface. The dilution of the lumen surface might be an explanation. This may cause less imide groups to be available for reaction with pei.

Figures $7 \mathrm{e}$ and $7 \mathrm{f}$ show the ATR-FTIR spectra for the fibers spun with $25 \mathrm{wt} \%$ (F7) and $28 \mathrm{wt} \%$ (F8) PEI in the dope solution; F1 is added as comparison. Figure 7e (lumen surface) shows that F8 has the highest absorbance in Box 1. However, in Boxes 2 and 3 there is little difference between the fibers. This suggests that there might be a limited amount of either PEI or pei available for crosslinking, as it seems that F8 has more free amines than F7 or F1. In Figure 7f, which displays the spectra of the outer surfaces, the spectrum of F8 looks very similar to that of neat PEI, indicating that there is hardly any crosslinking on the outer surface. The spectra of F7 has smaller amine and amide peaks than F1, but higher than F8 in the outer surface. When comparing the lumen and outer surface spectra of F7, the amine and amide peaks are slightly lower in the outer surface spectrum, indicating more crosslinking on the lumen surface. Based on these results, the crosslinking appears to be dependent on the concentration of PEI in the dope solution. When comparing F7 with F8, a higher concentration of PEI in the dope solution prevents the crosslinker from migrating to the outer surface. It is known that an increase in polymer concentration in the dope solution increases the overall density of the membrane and 
thus restricts pore size. This limits the ability of pei to migrate from the bore solution through the wall to the outer surface.

\subsubsection{Gel content}

To better investigate the crosslinking of the fibers, their gel content was measured. This was done by dissolving a known mass of fiber in NMP for 2 days, see Figure 8 . The residue, which consists of only the crosslinked part, was then thoroughly washed with water and dried in an oven at 37 ${ }^{\circ} \mathrm{C}$. Figure 9 presents the results of gel content, as well as SEM images of each fiber after gel content determination.

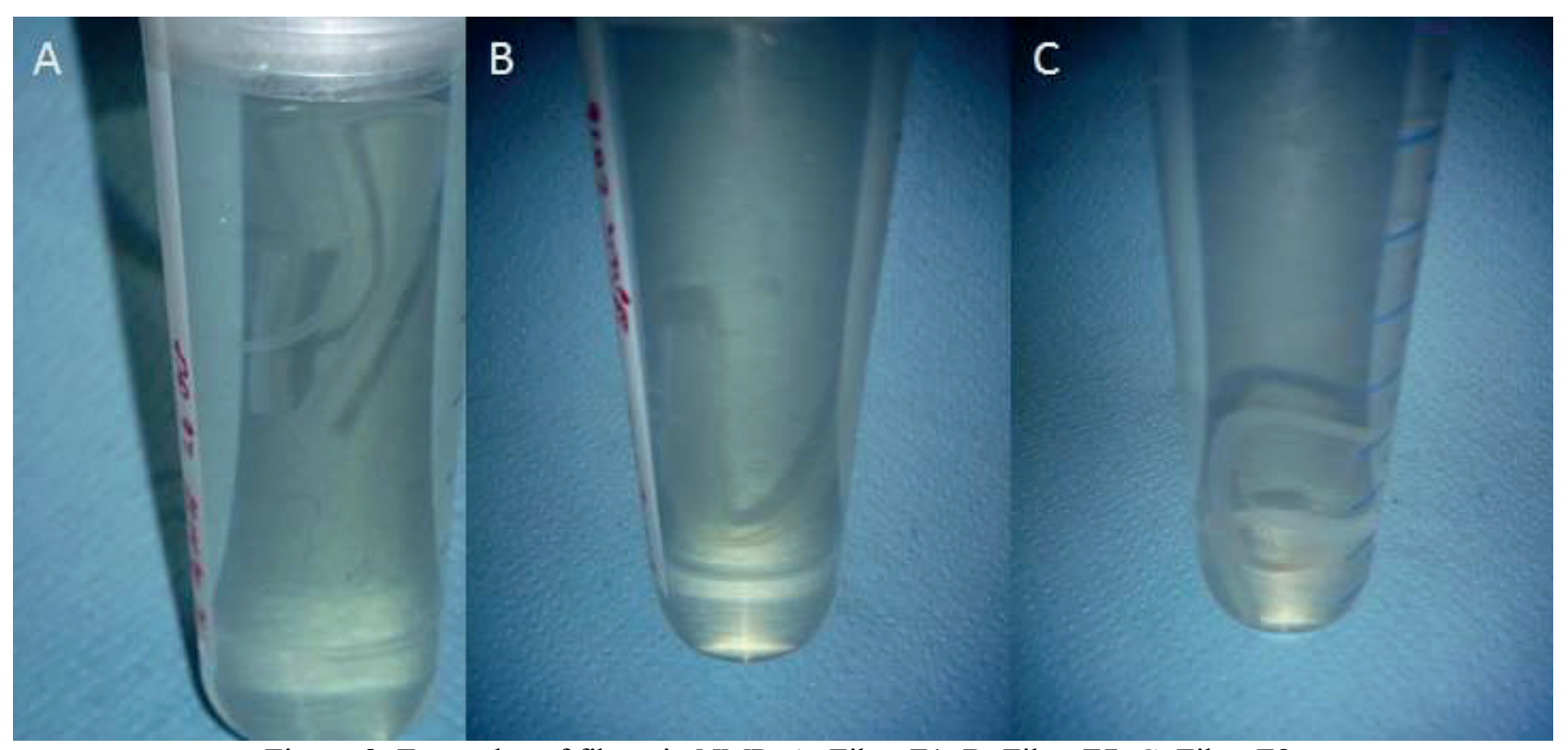

Figure 8: Examples of fibers in NMP. A: Fiber F1. B: Fiber F7. C: Fiber F8.

From the SEM images (Figure 9) it can be seen that the residues have round shape. This suggests that the crosslinking occurs in a homogeneous fashion all around the fibers. Figure 9a shows the results of the gel content measurement. The asterisk marks a statistically significant difference at $\mathrm{p}<0.05$, calculated using one-way ANOVA at $\mathrm{p}<0.05$ followed by Tukey's test, also at $\mathrm{p}<$ 0.05. All fibers were found to have significantly different gel contents. A trend can be seen in the results, with the gel content decreasing (lower fiber crosslinking) as the concentration of PEI in 
the dope solution increases. This can be explained by the higher membrane density resulting from higher dope concentrations which decreases the ability of the crosslinker to diffuse into the polymer matrix. This result is consistent to the findings of ATR-FTIR (Figure 7e and 7f), where for F8 crosslinking was not observed at the outer surface.
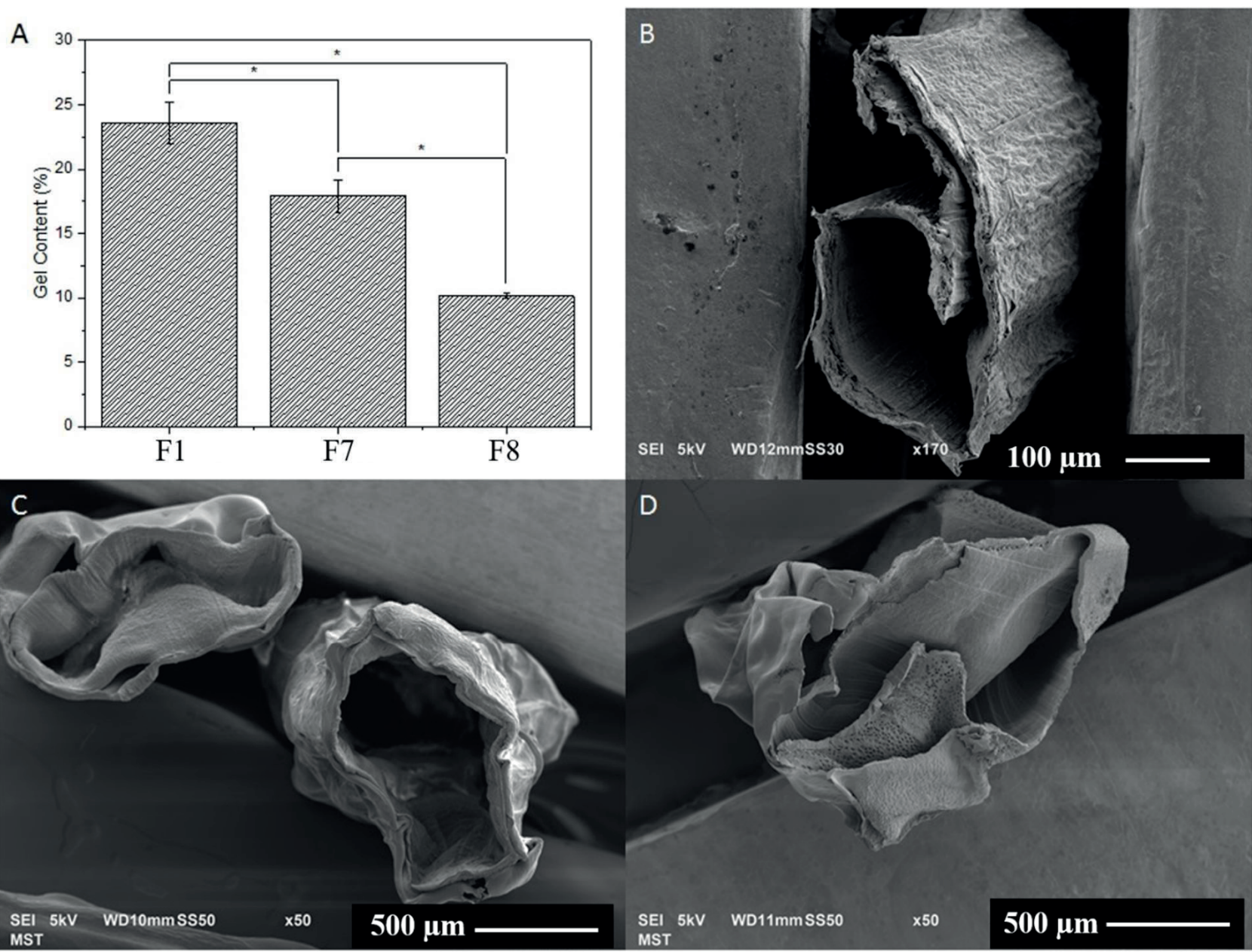

Figure 9. A) Gel content determination. Statistical significance is marked with $p<0,05$. B, C and D display SEM images of the residue after dissolution in NMP for F1 (B), F7 (C) and F8 (D), respectively.

\subsubsection{Zeta potential}

Zeta potential measurements were performed on F8 to measure the surface charge of the membrane. The inside surface charge of two samples of the same fiber was measured and the results are displayed in Figure 10. The fiber isoelectric point is at $\mathrm{pH} 9.9$ and till that value the surface charge is positive with a maximum around $22 \mathrm{mV}$. It is known that unmodified PEI is negatively charged, while the crosslinking with pei of various molecular weights causes the 
charge to become positive $[38,47,48]$. Therefore, the positive charge here can be attributed to the crosslinking of PEI with pei.

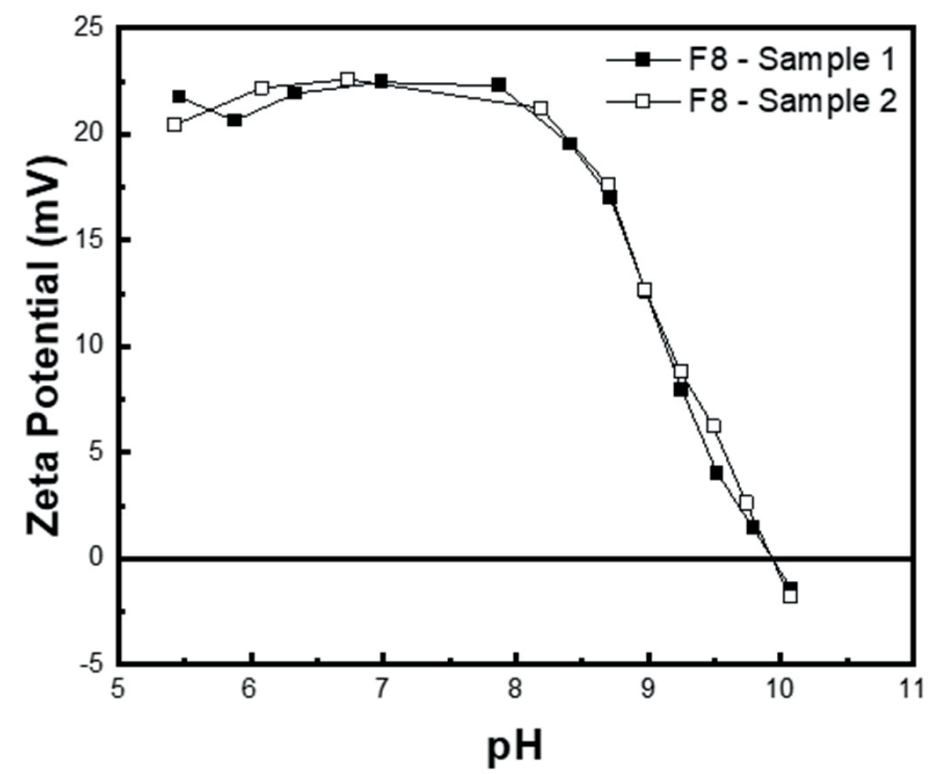

Figure 10: Zeta potential is plotted against the $\mathrm{pH}$ for two samples of Fiber 8.

\subsection{Membrane transport properties}

\subsubsection{Water transport}

The water transport across the produced fibers was also measured. Figure 11 shows the graphs of water flux versus TMP and Table 3 presents the water permeance estimated from the slopes.

Table 3. Water permeance of fibers F1-F8

\begin{tabular}{|c|c|}
\hline Fiber & $\begin{array}{c}\text { Clean Water Permeance } \\
\left(\mathbf{L} /\left(\mathbf{m}^{2} \cdot \mathbf{h} \cdot \text { Bar }\right)\right.\end{array}$ \\
\hline F1 & $75.3 \pm 11.0$ \\
\hline F2 & $124.1 \pm 16.3$ \\
\hline F3 & $104.5 \pm 34.1$ \\
\hline F4 & $81.6 \pm 26.7$ \\
\hline F5 & $107.7 \pm 29.7$ \\
\hline F6 & --- \\
\hline F7 & $7.0 \pm 0.9$ \\
\hline F8 & $0.03 \pm 0.00$ \\
\hline
\end{tabular}


a)

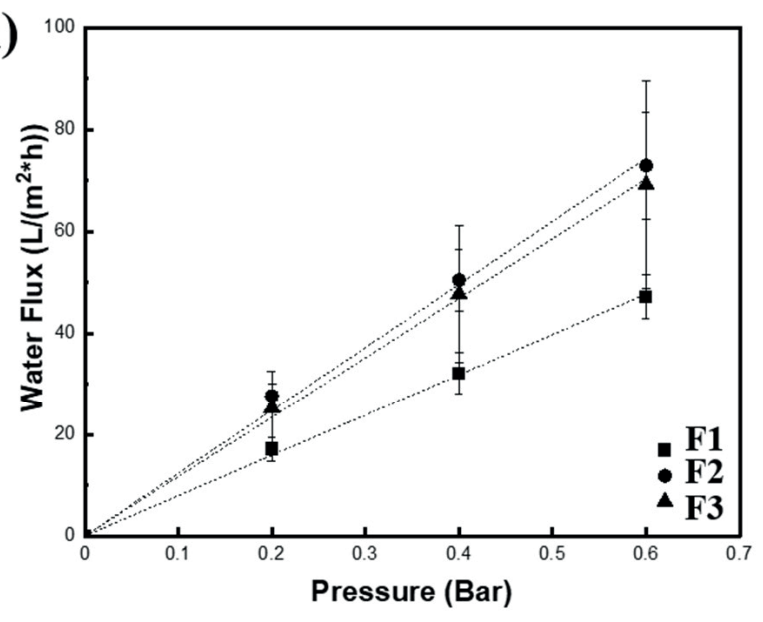

b)

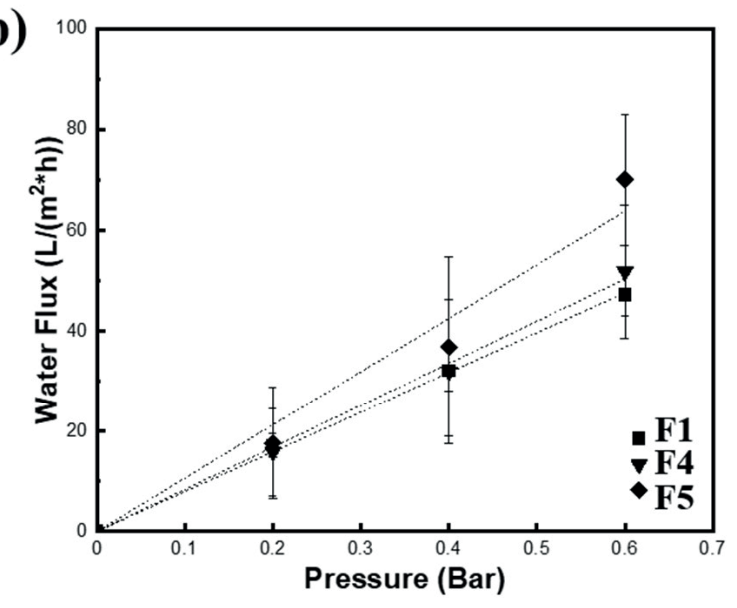

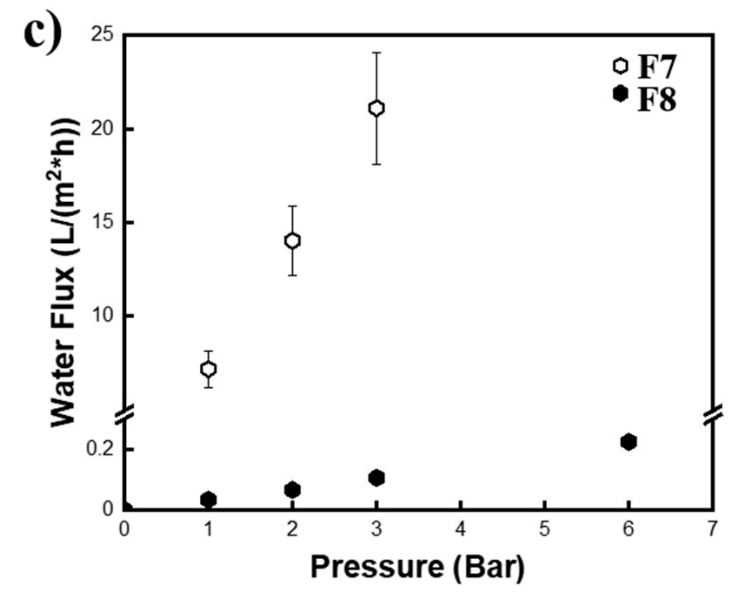

Figure 11: Water flux of the membranes versus TMP. a) F1-F3, comparison of the water flux of fibers spun at different bore flow rates; b) F1, F4 and F5, comparison of the water flux of fibers spun with different NMP concentrations in the bore solution; c) F7 and F8, comparison of the water flux of fibers spun with different concentrations of PEI in the dope solution.

The flux versus TMP is linear for all the fibers (except for F5) suggesting that no membrane compaction occurs at the TMP ranges used. No significant differences are observed for fibers F1-F5 (Figures 11a and 11b) suggesting that different bore flow rates and different NMP concentrations in the bore solution do not have an effect on membrane water flux. F6 could not be measured, as the fiber broke during handling. Figure $11 \mathrm{c}$ presents the water transport of F7 and F8 spun with different amounts of PEI in the dope solution. The water permeance of F8 is almost 200 times lower than that of F7. The values suggest that both are nanofiltration membranes. It is known that an increase in polymer concentration in the dope can increase the density of the membrane, thereby decreasing the membrane permeability. Moreover, in this work, the lumen surface is also crosslinked. 
Because the fibers spun with different bore flow rates (F1-F3) and different concentrations of solvent in the bore solution (F4 and F5) have water permeance much higher than those expected for nanofiltration membranes, they were not further investigated for the removal of ammonium ions.

\subsubsection{Ammonium acetate retention}

F7 and F8 have permeability in the nanofiltration range, therefore their retention of ammonium ions was tested in dead-end configuration. As a model solution, an aqueous solution of $40 \mathrm{mM}$ ammonium acetate was used. Figure 12 presents the results of F8, whereas F7 had no retention of ammonium ions.

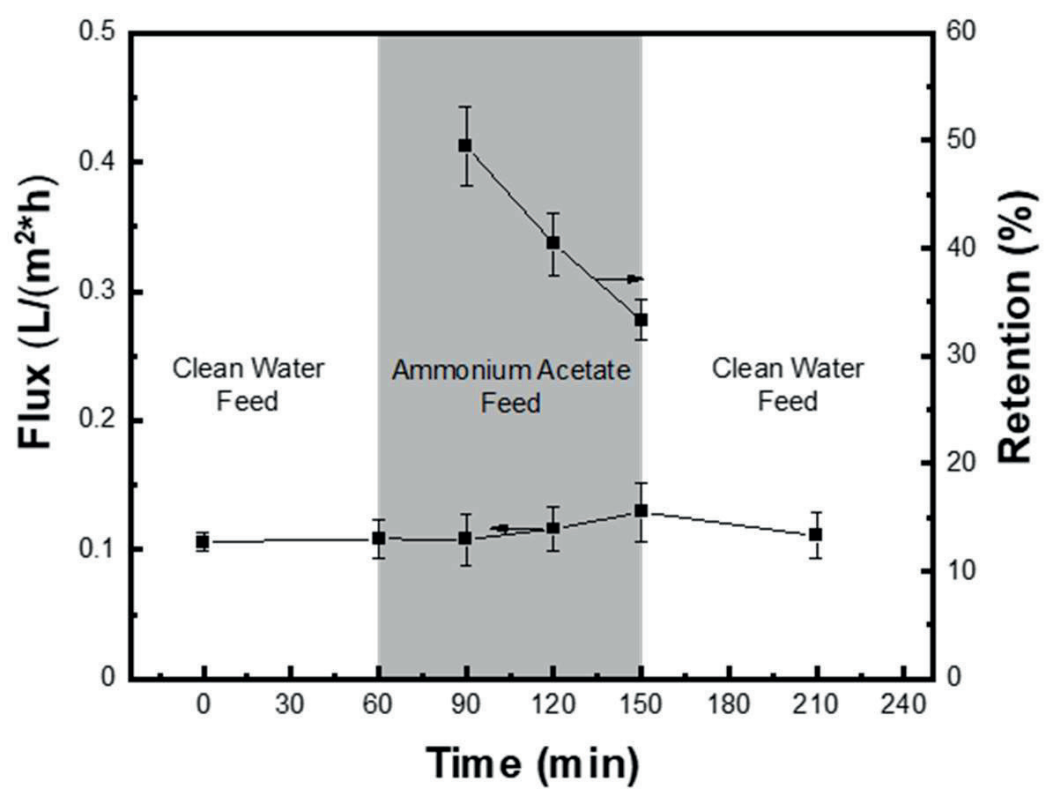

Figure 12: Results of ammonium acetate retentions experiments performed on F8. The grey area highlights the time in which ammonium acetate solution was used as feed.

F8 shows retention of $50 \%$ at 90 minutes from the start of the experiment, which decreased to $33 \%$ after 150 minutes. During the experiment, the concentration of ions in the lumen of the fibers increases, due to the continuous rejection of ions by the membrane. This may cause the charges on the lumen surface to become more shielded by the acetate counterions, reducing Donnan exclusion and resulting to decrease of ammonium ion retention [34,35]. 


\section{Conclusions and outlook}

In this work, positively-charged nanofiltration hollow fiber membranes were fabricated for the removal of ammonium ions from aqueous solutions. PEI was used as the membrane forming material and it was crosslinked with pei, in a process called 'Chemistry in the Spinneret', where the crosslinking occurs during the fiber spinning. The new membranes had positively charged lumen surface. The influence of the bore flow rate, amount of solvent in the bore solution and polymer concentration in the dope solution on the membrane morphology and performance was investigated. For all produced fibers crosslinking occurred, as shown by infrared spectroscopy and gel content determination. The fiber with the lowest water permeance $\left(0.03 \mathrm{~L} /\left(\mathrm{m}^{2} \cdot \mathrm{h} \cdot \mathrm{Bar}\right)\right)$ showed a retention of ammonium ions of approximately $50 \%$ after 30 minutes. However, the rejection decreased to $33 \%$ after 1.5 hours filtration, probably due to the shielding of the positive charges on the lumen surface.

Future works should focus on increasing the density of positive charges on the lumen of the fiber as well as increasing the membrane water permeance. Finally, studies should be performed using dialysate solution to investigate the effects of other components there on ammoniums ions rejection as a potential strategy to remove urea from spent dialysate.

\section{Acknowledgments}

This work was supported by the European Union's Horizon 2020 research and innovation program under the Marie Skłodowska-Curie grant agreement no. 642890 (http://thelinkproject.eu/). The European membrane Institute Twente (EMI) is acknowledged for the support with the zeta potential measurements. 


\section{References}

[1] C. Zoccali, U. Moissl, C. Chazot, F. Mallamaci, G. Tripepi, O. Arkossy, P. Wabel, S. Stuard, Chronic fluid overload and mortality in ESRD, Journal of the American Society of Nephrology 28(8) (2017) 24912497.

[2] W.R. Clark, N.L. Dehghani, V. Narsimhan, C. Ronco, Uremic toxins and their relation to dialysis efficacy, Blood Purification 48(4) (2019) 299-314.

[3] J.W.M. Agar, Green dialysis: The environmental challenges ahead, Seminars in Dialysis 28(2) (2015) 186-192.

[4] C. Boccato, D.W. Evans, R. Lucena, J.r. Vienken, Water and dialysis fluids: a quality management guide, Pabst Science Publishers, 2015.

[5] J. Himmelfarb, R. Vanholder, R. Mehrotra, M. Tonelli, The current and future landscape of dialysis, Nature Reviews Nephrology 16(10) (2020) 573-585.

[6] W.H. Fissell, S. Roy, A. Davenport, Achieving more frequent and longer dialysis for the majority: wearable dialysis and implantable artificial kidney devices, Kidney International 84(2) (2013) 256-264.

[7] M.K. van Gelder, S.M. Mihaila, J. Jansen, M. Wester, M.C. Verhaar, J.A. Joles, D. Stamatialis, R. Masereeuw, K.G.F. Gerritsen, From portable dialysis to a bioengineered kidney, Expert Review of Medical Devices 15(5) (2018) 323-336.

[8] P. Armignacco, A. Lorenzin, M. Neri, F. Nalesso, F. Garzotto, C. Ronco, Wearable devices for blood purification: principles, miniaturization, and technical challenges, Seminars in Dialysis 28(2) (2015) 125130.

[9] P. Armignacco, F. Garzotto, M. Neri, A. Lorenzin, C. Ronco, Wak engineering evolution, Blood purification 39(1-3) (2015) 110-114.

[10] M.K. van Gelder, J.A.W. Jong, L. Folkertsma, Y. Guo, C. Blüchel, M.C. Verhaar, M. Odijk, C.F. Van Nostrum, W.E. Hennink, K.G.F. Gerritsen, Urea removal strategies for dialysate regeneration in a wearable artificial kidney, Biomaterials 234 (2020) 119735. 
[11] I.D. Weiner, W.E. Mitch, J.M. Sands, Urea and ammonia Metabolism and the control of renal nitrogen excretion, Clinical journal of the American Society of Nephrology 10(8) (2015) 1444-1458.

[12] M. Roberts, The regenerative dialysis (REDY) sorbent system, Nephrology 4(4) (1998) 275-278.

[13] M. Wester, F. Simonis, N. Lachkar, W.K. Wodzig, F.J. Meuwissen, J.P. Kooman, W.H. Boer, J.A. Joles, K.G. Gerritsen, Removal of urea in a wearable dialysis device: a reappraisal of electro-oxidation, Artificial Organs 38(12) (2014) 998-1006.

[14] J.C. Wright, A.S. Michaels, A.J. Appleby, Electrooxidation of urea at the ruthenium titanium oxide electrode, American Institute of Chemical Engineers Journal 32(9) (1986) 1450-1458.

[15] B. Schuenemann, E. Quellhorst, H. Kaiser, G. Richter, K. Mundt, E. Weidlich, G. Loeffler, M. Zachariae, O. Schunk, Regeneration of filtrate and dialysis fluid by electro-oxidation and absorption, American Society for Artificial Internal Organans Journal 28(1) (1982).

[16] M. Wester, M.K. van Gelder, J.A. Joles, F. Simonis, D.H.M. Hazenbrink, T.W.M. van Berkel, K.R.D. Vaessen, W.H. Boer, M.C. Verhaar, K.G.F. Gerritsen, Removal of urea by electro-oxidation in a miniature dialysis device: a study in awake goats, American Journal of Physiology - Renal Physiology 315(5) (2018) 1385-1397.

[17] C.H. Ooi, W.K. Cheah, Y.L. Sim, S.Y. Pung, F.Y. Yeoh, Conversion and characterization of activated carbon fiber derived from palm empty fruit bunch waste and its kinetic study on urea adsorption, Journal of Environmental Management 197 (2017) 199-205.

[18] Y.C. Cheng, C.C. Fu, Y.S. Hsiao, C.C. Chien, R.S. Juang, Clearance of low molecular-weight uremic toxins $\mathrm{p}$-cresol, creatinine, and urea from simulated serum by adsorption, Journal of Molecular Liquids 252 (2017).

[19] J.H. Kim, J.C. Kim, J.H. Moon, J.Y. Park, K.K. Lee, E. Kang, H.C. Kim, B.G. Min, C. Ronco, Development of a cold dialysate regeneration system for home hemodialysis, Blood Purification 28(2) (2009) 84-92.

[20] C. Giordano, R. Esposito, P. Bello, E. Quarto, F.M. Gonzalez, Cold carbon apparatus for hemodialysis, Journal of Dialysis 1(2) (1976) 165-179. 
[21] W.K. Cheah, Y.L. Sim, F.Y. Yeoh, Amine-functionalized mesoporous silica for urea adsorption, Materials Chemistry and Physics 175 (2016) 151-157.

[22] V. Wernert, O. Schäf, H. Ghobarkar, R. Denoyel, Adsorption properties of zeolites for artificial kidney applications, Microporous and Mesoporous Materials 83(1) (2005) 101-113.

[23] F. Meng, M. Seredych, C. Chen, V. Gura, S. Mikhalovsky, S. Sandeman, G. Ingavle, T. Ozulumba, L. Miao, B. Anasori, Y. Gogotsi, MXene sorbents for removal of urea from dialysate: a step toward the wearable artificial kidney, ACS Nano 12(10) (2018) 10518-10528.

[24] J.A.W. Jong, R. Smakman, M.E. Moret, M.C. Verhaar, W.E. Hennink, K.G.F. Gerritsen, C.F. Van Nostrum, Reactivity of (vicinal) carbonyl compounds with urea, ACS Omega 4(7) (2019) 11928-11937.

[25] T. Shimizu, S. Fujishige, A newly prepared surface-treated oxystarch for removal of urea, Journal of Biomedical Materials Research 17(4) (1983) 597-612.

[26] D. Deepak, Evaluation of adsorbents for the removal of metabolic wastes from blood, Medical and Biological Engineering and Computing 19(6) (1981) 701-706.

[27] M.N.Z. Abidin, P.S. Goh, A.F. Ismail, N. Said, M.H.D. Othman, H. Hasbullah, M.S. Abdullah, B.C. Ng, S.H.S.A. Kadir, F. Kamal, Highly adsorptive oxidized starch nanoparticles for efficient urea removal, Carbohydrate Polymers 201 (2018) 257-263.

[28] J.A.W. Jong, Y. Guo, C. Veenhoven, M.E. Moret, J. van der Zwan, A. Lucini Paioni, M. Baldus, K.C. Scheiner, R. Dalebout, M.J. van Steenbergen, M.C. Verhaar, R. Smakman, W.E. Hennink, K.G.F. Gerritsen, C.F. van Nostrum, Phenylglyoxaldehyde-functionalized polymeric sorbents for urea removal from aqueous solutions, ACS Applied Polymer Materials 2(2) (2020) 515-527.

[29] J.A.W. Jong, Y. Guo, D. Hazenbrink, S. Douka, D. Verdijk, J. van der Zwan, K. Houben, M. Baldus, K.C. Scheiner, R. Dalebout, M.C. Verhaar, R. Smakman, W.E. Hennink, K.G.F. Gerritsen, C.F. van Nostrum, A ninhydrin-type urea sorbent for the development of a wearable artificial kidney, Macromolecular Bioscience 20(3) (2020) e1900396.

[30] R. Smakman, A.W. van Doorn, Urea removal by means of direct binding, Clinical nephrology 26 (Suppl.1) (1986) 58-62. 
[31] R. Shapiro, N. Chatterjie, Cyclization reactions of ninhydrin with aromatic amines and ureas, The Journal of Organic Chemistry 35(2) (1970) 447-450.

[32] J. Schaep, B. Van der Bruggen, C. Vandecasteele, D. Wilms, Influence of ion size and charge in nanofiltration, Separation and Purification Technology 14(1) (1998) 155-162.

[33] S. Cheng, D.L. Oatley, P.M. Williams, C.J. Wright, Positively charged nanofiltration membranes: review of current fabrication methods and introduction of a novel approach, Advances in Colloid and Interface Science 164(1) (2011) 12-20.

[34] Z. Qin, C. Geng, H. Guo, Z. Du, G. Zhang, S. Ji, Synthesis of positively charged polyelectrolyte multilayer membranes for removal of divalent metal ions, Journal of Materials Research 28(11) (2013) 1449-1457.

[35] G. Liu, D.M. Dotzauer, M.L. Bruening, Ion-exchange membranes prepared using layer-by-layer polyelectrolyte deposition, Journal of Membrane Science 354(1-2) (2010) 198-205.

[36] Y. Zheng, G. Yao, Q. Cheng, S. Yu, M. Liu, C. Gao, Positively charged thin-film composite hollow fiber nanofiltration membrane for the removal of cationic dyes through submerged filtration, Desalination 328 (2013) 42-50.

[37] T.K. Dey, R.C. Bindal, S. Prabhakar, P.K. Tewari, Development, characterization and performance evaluation of positively-charged thin film-composite nanofiltration membrane containing fixed quaternary ammonium moieties, Separation Science and Technology 46(6) (2011) 933-943.

[38] C. Trimpert, G. Boese, W. Albrecht, K. Richau, T. Weigel, A. Lendlein, T. Groth, Poly(ether imide) membranes modified with poly(ethylene imine) as potential carriers for epidermal substitutes, Macromolecular Bioscience 6(4) (2006) 274-284.

[39] C. Ba, J. Langer, J. Economy, Chemical modification of P84 copolyimide membranes by polyethylenimine for nanofiltration, Journal of Membrane Science 327 (2009) 49-58.

[40] J. Gao, S.-P. Sun, W.-P. Zhu, T.-S. Chung, Polyethyleneimine (PEI) cross-linked P84 nanofiltration (NF) hollow fiber membranes for Pb2+ removal, Journal of Membrane Science 452 (2014) 300-310. 
[41] K. Vanherck, A. Cano-Odena, G. Koeckelberghs, T. Dedroog, I. Vankelecom, A simplified diamine crosslinking method for PI nanofiltration membranes, Journal of Membrane Science 353(1) (2010) 135 143.

[42] K.K. Kopeć, S.M. Dutczak, M. Wessling, D.F. Stamatialis, Chemistry in a spinneret-On the interplay of crosslinking and phase inversion during spinning of novel hollow fiber membranes, Journal of Membrane Science 369(1) (2011) 308-318.

[43] Q.F. Alsalhy, H.A. Salih, R.H. Melkon, Y.M. Mahdi, N.A. Abdul Karim, Effect of the preparation conditions on the morphology and performance of poly(imide) hollow fiber membranes, Journal of Applied Polymer Science 131(12) (2014).

[44] G. Dong, H. Li, V. Chen, Factors affect defect-free Matrimid® hollow fiber gas separation performance in natural gas purification, Journal of Membrane Science 353(1) (2010) 17-27.

[45] W. Albrecht, T. Weigel, M. Schossig-Tiedemann, K. Kneifel, K.V. Peinemann, D. Paul, Formation of hollow fiber membranes from poly(ether imide) at wet phase inversion using binary mixtures of solvents for the preparation of the dope, Journal of Membrane Science 192(1) (2001) 217-230.

[46] G. Bakeri, A.F. Ismail, M. Shariaty-Niassar, T. Matsuura, Effect of polymer concentration on the structure and performance of polyetherimide hollow fiber membranes, Journal of Membrane Science 363(1) (2010) 103-111.

[47] S. Gassara, W. Chinpa, D. Quemener, R. Ben Amar, A. Deratani, Pore size tailoring of poly(ether imide) membrane from UF to NF range by chemical post-treatment using aminated oligomers, Journal of Membrane Science 436 (2013) 36-46.

[48] C. Feng, K. Khulbe, G. Chowdhury, T. Matsuura, V.C. Sapkal, Structural and performance study of microporous polyetherimide hollow fiber membranes made by solvent-spinning method, Journal of Membrane Science 189 (2001) 193-203. 



\title{
Chapter 7
}

\section{Conclusions and Outlook}

\author{
I. Geremia and D. Stamatialis
}

(Bio)artificial organs, Department of Biomaterials Science and Technology, TechMed Centre, Faculty of Science and Technology, University of Twente, P.O. Box 217, 7500 AE Enschede, The Netherlands 
In this thesis we investigated new membrane strategies for improved blood purification and spent dialysate regeneration in hemodialysis (HD). Specific attention was focused on strategies which could provide better and more continuous therapy, as well as strategies for efficient spent dialysate regeneration, which is crucial for the development of portable (PAK) and wearable (WAK) artificial kidney devices.

Continuous therapies would significantly improve the removal of toxins from the patient's blood and would lead to a better control of body water volumes as they overcome the intermittent character of standard HD therapy [1-6]. PAK and WAK, moreover, would ameliorate the patients' quality of life, as they would improve mobility and would allow participation to social life. Importantly these systems, which require low water consumption, would contribute to greener dialysis therapy.

In Chapters 2, 3 and 4 we developed membranes for better and preferably long-term blood purification. More specific, in Chapters 2 and 3 a Mixed Matrix Membrane (MMM) composed of activated carbon (AC) embedded in a polyethersulfone (PES)/polyvinylpyrrolidone (PVP) polymer matrix (AC/PES/PVP MMM) is investigated. In Chapter 2 we focused on the risk related to the bacteria contamination of the HD system during long-term therapy and on the ability of the MMM to simultaneously purify blood plasma and remove endotoxins from the dialysate, combined to acting as a safe barrier to pyrogens from the dialysate. In Chapter 3, we proved the excellent hemocompatibility profile of the MMM, which is a key requisite for better therapies. In Chapter 4 we discussed the development of hollow fibers (HF) for outside-in filtration (OIF) which could contribute to longer therapy by reducing the risk of fiber clotting by blood thrombi [7].

In Chapters 5 and 6, we focused on the development of membrane based strategies for the removal of urea from spent dialysate, which is one of the main challenges hampering nowadays the clinical implementation of dialysate regeneration systems in PAK and WAK [8]. Here, we proposed two strategies for the removal of urea from the dialysate. One, described in Chapter 5, concerns the use of a MMM based on PES/PVP and ninhydrin-polystyrene (PS-Nin) particles (PS-Nin MMM) which, 
thanks to the presence of ninhydrin moieties, can covalently bind urea. In Chapter 6, we studied the development of a positively-charged nanofiltration hollow fiber (PCN) membrane for the retention of ammonium ions obtained by the hydrolysis of urea by means of ureases.

In the next sections we will discuss the main conclusions, limitations and outlook of the work presented in the different scientific chapters.

\section{Mixed matrix membrane for improved blood purification and dialysate depyrogenation}

In Chapter 2 we have shown that the AC/PES/PVP MMM can simultaneously remove protein bound uremic toxins (PBTs) from blood plasma and endotoxins (lipopolysaccharide) from dialysate. Importantly, this MMM can remove approximately 10 times more endotoxins compared to HD commercial membranes and can act as a safety barrier to avoid the transport of endotoxins from the dialysate to the blood. This is an important achievement as it may open the way towards the future investigation of simultaneous purification of blood and dialysate using a single membrane. Based on our results, we have estimated that we would need quite low membrane surface area of $0.15 \mathrm{~m}^{2}$ for the removal of the daily production of indoxyl sulphate (IS) and hippuric acid (HA) while simultaneously depyrogenating the dialysate. This MMM could be suitable for a WAK/PAK device. In this work we have performed the simultaneous plasma and dialysate purification study only for 4 hours, therefore not simulating a prolonged treatment. However, we found out that the MMM was not saturated within this period (Figure 1) and could be potentially used for longer time. 
a)
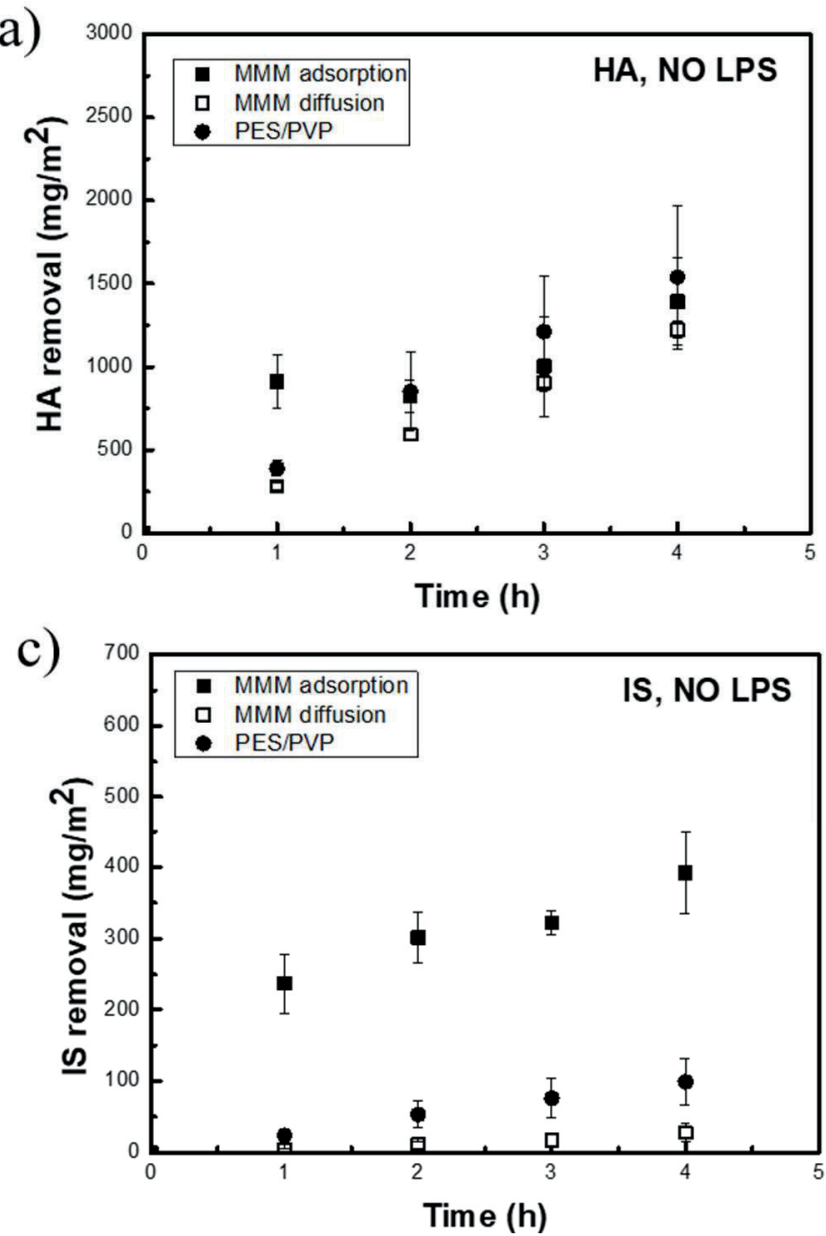

b)

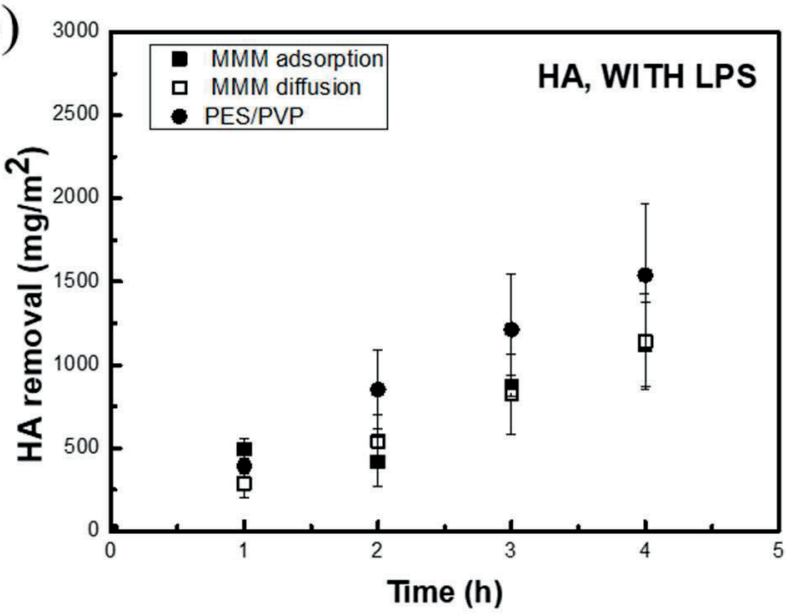

d)

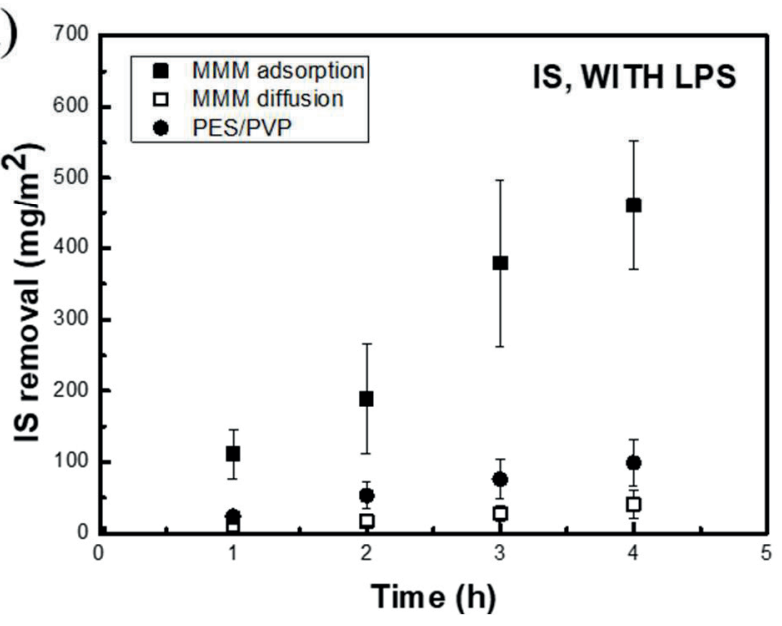

Figure 1. Adsorption and diffusion contributions of AC/PES/PVP Mixed Matrix Membrane (MMM) on hippuric acid (HA) removal and comparison with PES/PVP control hollow fiber without (a) and with (b) simultaneous lipopolysaccharide (LPS) removal from the dialysate. Adsorption and diffusion contributions of AC/PES/PVP Mixed Matrix Membrane (MMM) on indoxyl sulphate (IS) removal and comparison with PES/PVP control hollow fiber without (c) and with (d) simultaneous lipopolysaccharide (LPS) removal from the dialysate.

Therefore, the simultaneous removal experiment should be performed in the future for longer time. Of note, in long term experiments, the risk of dialysate contamination is higher and, therefore, it would also better simulate real conditions in prolonged therapies. Besides, here we have performed the study using human plasma spiked only with 2 PBTs (IS and HA). In the future, one should test the simultaneous blood/dialysate purification using patient plasma and full blood to study saturation of $\mathrm{AC}$ in this more complex situation. Furthermore, we have used a miniaturized set-up, with a small size module and plasma and dialysate flow rates much lower compared to those used in the clinics. The study should be repeated using a scaled-up module and flow rates similar to those applied in the clinic. One could anticipate that higher flow rates would lead to higher removal of uremic toxins from 
blood and endotoxins from dialysate by increasing the number of runs through the AC/PES/PVP MMM HF and by reducing fouling and concentration polarization phenomena.

An ultra-pure dialysate model solution spiked artificially with pyrogens was used in this study. In this way, we could not investigate the effect of the presence of uremic toxins in the dialysate which may compete with endotoxins for adsorption on the AC. Therefore, endotoxins removal experiments should also be performed in the future using spent dialysate derived from patients.

The most common concentrations of endotoxins found in tap water are in the range 7-8 EU/mL [911], therefore quite close to $10 \mathrm{EU} / \mathrm{mL}$ we used in the simultaneous plasma/dialysate purification experiment. One could anticipate that the MMM could be suitable for HD applications using dialysate directly prepared from tap water. It would be interesting to investigate the endotoxins removal there (with and without simultaneous plasma purification) and investigate possible effects deriving from the presence of minerals and other components of tap water. This application would be very relevant, especially for developing countries where often the quality of water used for the preparation of the dialysate is not good [12] and for emergency situations when there is no time/resources to purify water for the preparation of the dialysate.

Besides HD, there might be other applications where this MMM could be applied, as for example for the removal of endotoxins from medical/pharmacological preparations. In this contest, it could be used for the purification of the water used for the preparation of drugs (as a safety check point before the addition of the drugs constituents) to ensure that no pyrogens would be present in the final preparation.

Finally, the MMM could also be applied in sepsis for the removal of endotoxins and cytokines from patients' blood [13]. However, for this, the molecular weight cut-off (MWCO) of the membrane (approximately $12 \mathrm{KDa}$ ) needs to be adapted to allow the transport of endotoxins and cytokines through the selective layer. A recent study of Kim et al showed the development of a MMM with MWCO of $47 \mathrm{KDa}$ and $99 \%$ bovine serum albumin retention and narrow pore size distribution [14]. This MMM could be relevant for application to sepsis. 
In Chapter 3 the hemocompatibility profile of the AC/PES/PVP MMM was studied and we found that it is comparable to that of HD membranes currently applied in the clinic. The good hemocompatibility of the AC/PES/PVP MMM is due to the PVP-enriched lumen surface which prevents plasma proteins adsorption, to the smoothness of the lumen surface which avoids entrapment/damage of blood cells and platelets and to the selective lumen layer which prevents blood to contact with the AC. The hemocompatibility profile of the MMM was also compared to that of a previous generation high-flux MMM. For the latter, the activation of the coagulation pathway was observed, most probably due to the open selective layer which leads to the contact of blood with AC. The reduction of erythrocytes, white blood cells and platelets there was attributed to the high surface roughness of the lumen layer of the high-flux MMM which is expected to entrap and damage blood cells and platelets.

In this study the hemocompatibility experiments were performed only for 3 hours but, as we aim to apply the AC/PES/PVP MMM in PAK and WAK systems, the hemocompatibility should be assessed for longer time. Finally, as already mentioned for Chapter 2, we should perform the hemocompatibility experiments with scaled-up MMM modules to apply the same blood and dialysate flow rates as those applied in the clinics.

\section{Hollow fibers for Outside-In Filtration (OIF)}

In Chapter 4 we discussed the development of a new HF for applications in OIF HD with the enriched-PVP selective layer on the outside of the fiber, high ultrafiltration coefficient (KUf) and low albumin sieving coefficient. Also, the fiber diameter was small to achieve modules with high packing density. The developed protocol is simple and eco-friendly and presents many advantages compared to those already reported in literature [15-18]. For example, in our case the spinning occurs at room temperature, it requires only water in the coagulation bath without the need of solvents and it does 
not require a dedicated spinneret (a spinneret for the production of inside-out filtration fibers was used). A long-term blood purification experiment (possibly with uremic blood) in OIF configuration should be performed as here the OIF fiber was assessed only for creatinine removal from human plasma at 24 hours.

The developed OIF fiber is meant only for diffusion (and potentially convection) HD applications but it does not possess adsorption properties. Nevertheless, based on the exceptional results we have achieved for the AC/PES/PVP MMM (Chapter 2) [19-21], it would be interesting to investigate the development of MMM for OIF. Preliminary results on this have already been reported by Ter Beek et al. [22] who developed for the first time OIF MMM with superior performance in vitro for the removal of the PBTs IS and HA compared to the MMM designed for inside-out standard filtration mode. However, the selective layer of this OIF MMM is most probably at the lumen side. This might have many implications and drawbacks in term of hemocompatibility due to the risk of blood contact with the $\mathrm{AC}$ and blood cells and platelets entrapment in the porous outer membrane matrix. Therefore, future studies should investigate the combination of the protocols developed for the production of the OIF MMM [22] and for the spinning of the OIF fiber, described in our work, to obtain new OIF MMM with the selective layer on the outside.

\section{Urea removal from spent dialysate}

In Chapter 5 a new MMM based on PES/PVP and ninhydrin-enriched polystyrene particles (PSNin), named PS-Nin MMM, was developed for urea removal from spent dialysate. We have observed that the addition of small PS-Nin particles in the MMM increases urea removal kinetics and total urea removal in comparison to the particles in suspension. This is due to the good dispersion of the particles in the MMM without aggregation, which improves the accessibility of urea to the ninhydrin moieties. In recirculation dynamic experiments, the MMM can remove approximately $3.4 \mathrm{mmol}$ of urea per 
grams of particles after 4 hours, which is one of the highest urea removal values reported in literature. This urea removal is higher than the theoretical amount of urea expected if all the ninhydrin groups would react with urea. We hypothesise that this remarkable result is due to the physisorption (via hydrogen bonding) of urea on urea already covalently bound to the sorbent particles (chemisorption). Based on these results, we have estimated that $215 \mathrm{~g}$ of the MMM would be needed to remove the daily produced urea from spent dialysate, which is very promising for applications in PAK and WAK where lightness and miniaturization are essential.

Importantly, our work brings another confirmation that the incorporation of small particles in a MMM have positive effects in terms of solute removal compared to the particles alone in bed columns or in suspension, due to the good dispersion and absence of aggregation/agglomeration in the MMM. As a matter of fact, in an earlier study Tetala et al. [23] observed the superiority of a MMM composed of chitosan (CS) particles embedded in EVAL (ethylene vinyl alcohol) polymer matrix in copper ions removal compared to the same particles packed in bed columns. The better performance of the MMM there [23] was due to the better mass transfer within the MMM with lower pressure drop generation and to the fact that no particles aggregation and system plugging was observed compared to the bed column.

With regards to urea removal from dialysate, based on the high urea removal reported in literature for metal-chitosan (CS) coordination complexes [8, 24-27], we also investigated the development of MMM containing crosslinked-CS particles (55 wt \%) embedded in EVAL polymer matrix. CS was crosslinked with glutaraldehyde (using a protocol already reported in literature [26]) to avoid the formation of hydrogen bonds between CS chains thus leaving more amino groups on CS available for copper adsorption [26]. Copper (Cu (II)) adsorption on the MMM (Cu-CS-MMM) was performed to introduce favourable Lewis acid coordination sites for urea ligands, since it has been shown that urea could bind to unoccupied d-orbital of metal ions $[8,26]$. In Figure 2, we report an illustration of urea-Cu(II) coordination in CS-glutaraldehyde copolymer. Figure 3 shows a picture of the flat-sheet 
CS-MMM, SEM images of the cross-section of CS-MMM, comparison of copper adsorption on CS$\mathrm{MMM}$ and $\mathrm{CS}$ particles in suspension and urea adsorption on $\mathrm{Cu}-\mathrm{CS}-\mathrm{MMM}$ and on the $\mathrm{Cu}-\mathrm{CS}$ particles in suspension. Urea detection was performed using the same quantification method reported in Chapter 4 (enzymatic assay Urea FS* (Diasys, Holzheim, Germany)). As control, we tested copper and urea adsorption on EVAL membrane without particles: as expected, the results showed that EVAL membrane does not adsorb neither copper nor urea. As in the case of MMM with PS-Nin particles for urea adsorption (this study) and for the CS-EVAL MMM for copper adsorption [23], the MMM with $\mathrm{Cu}-\mathrm{CS}$ particles provided better urea removal compared to the particles in suspension (Figure 3e).

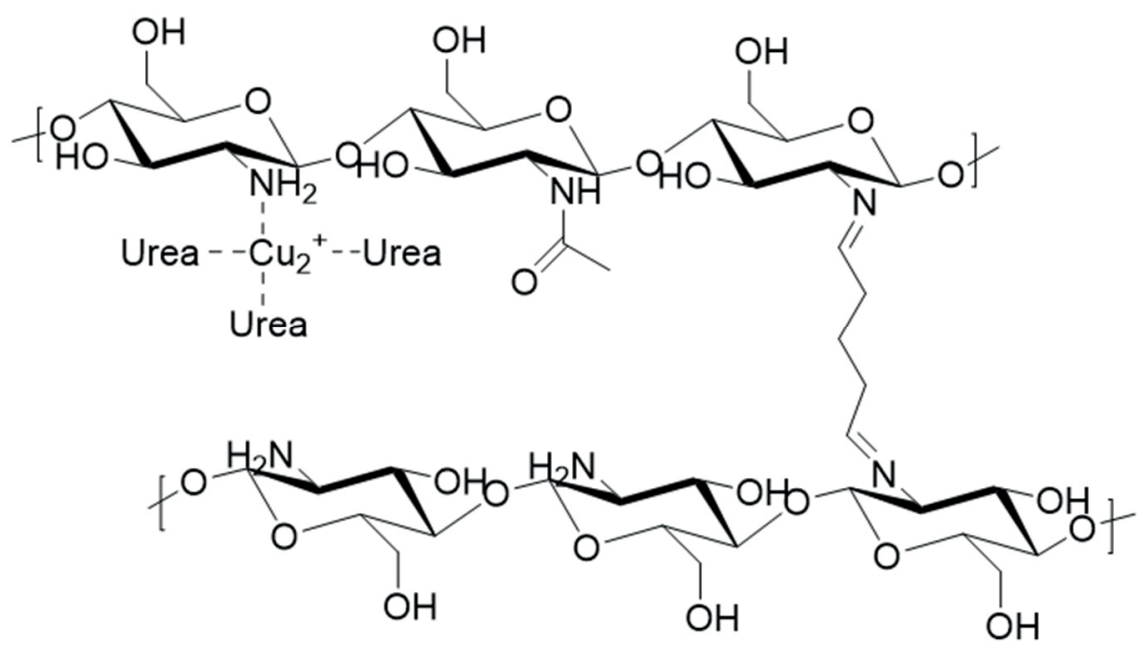

Figure 2. Illustration of urea-Cu(II) coordination in CS-glutaraldehyde copolymer ("pendant model"). The "bridge" model is another possible coordination model of $\mathrm{Cu}$ (II) in CS-glutaraldehyde copolymer, where $\mathrm{Cu}$ (II) binds to two amino groups of CS and to two molecules of urea [26]. 

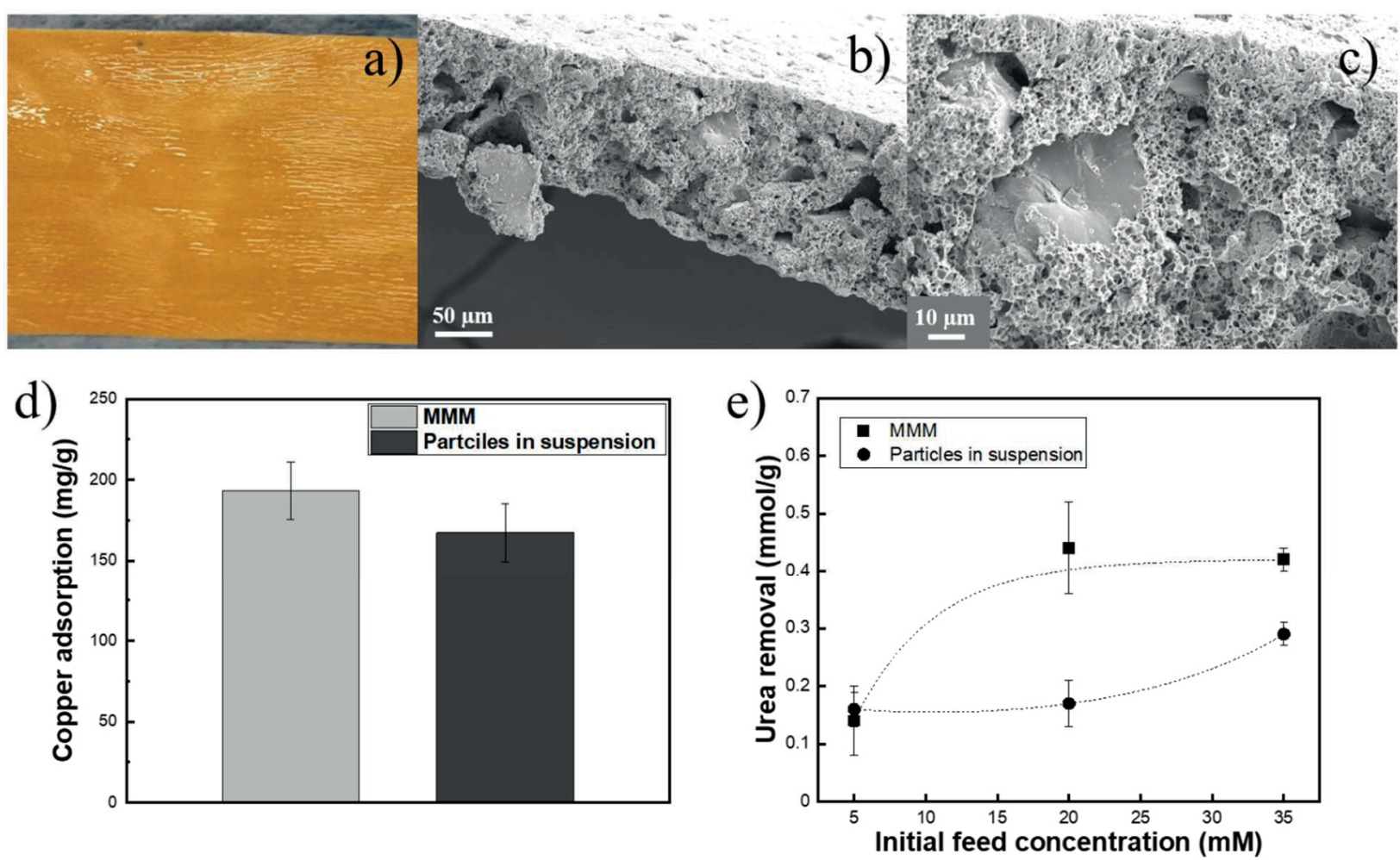

Figure 3. a) Picture of the CS- MMM (top layer); b) SEM image of the cross-section of the CS-MMM; c) SEM magnification of the cross-section of the CS-MMM; d) Copper adsorption by the CS-MMM (adsorption reported per grams of particles in the MMM) and CS particles in suspension (24h; $n=3$; Average \pm SD); e) Urea adsorption on Cu$\mathrm{CS}-\mathrm{MMM}$ (adsorption reported per grams of particles in the MMM) and $\mathrm{Cu}-\mathrm{CS}$ particles in suspension $(24 \mathrm{~h}$; $\mathrm{n}=3$; Average $\pm \mathrm{SD})$.

Using an initial urea concentration of $35 \mathrm{mM}$, we however obtained a urea removal equal to 0.4 $\mathrm{mmol} / \mathrm{g}$ (grams of CS-particles in the MMM), much lower than that reported for the Cu-CS sorbent systems in other studies [24-27]. For example, Zhou et al. [24] reported a urea removal value of 2.0 $\mathrm{mmol} / \mathrm{g}$ using microporous $\mathrm{Cu}-\mathrm{CS}$ particles and urea concentration equal to $22 \mathrm{mM}$. Wilson et al. [26] achieved $4.4 \mathrm{mmol} / \mathrm{g}$ of the same CS glutaraldehyde crosslinked copolymer as the one we used. It is still not clear why we could not achieve comparable urea removal results as Zhou et al. [24] and Wilson et al. [26]. In the work of Zhou et al. [24] the particle size of the sorbents is not reported, which, however, is an important factor affecting adsorption capacity. Moreover, they used a Nessler spectrophotometer method for urea quantification without reporting any detail on the experimental procedure. With regards to the work of Wilson et al. [26], they used urea concentration ranging from 1 to $30 \mathrm{mM}$ but the CS-urea ratio was not reported. Interestingly, in this work the molar ratio of amino 
groups of chitosan and aldehydes of glutaraldehyde was 1:2, meaning that theoretically all the amino groups of chitosan could have reacted with glutaraldehyde and converted into imines [8]. Moreover, urea quantification was performed by means of a very old spectrophotometer detection method using p-dimethyl-aminobenzaldehyde, which may be altered by the presence of other amino species [28]. This aspect should be better investigated due to the presence of amino groups on the CS chains.

With regards to our newly developed PS-Nin MMM, the urea removal results are very good. Future works should focus on optimizing the spinning conditions in order to obtain standard round-shaped lumen and also smaller dimensions (preferably smaller inner diameter) of the HF as this would allow to increase the fiber packing density in the module. Due to time limitation and limited amount of particles this was not been possible during the doctorate. In order to achieve a round lumen shape, the polymer dope flow rate or the polymer dope concentration should be increased. Other strategies may be exploited to reduce the overall size of the HF. A reduction of the bore flow rate is expected to decrease the inner diameter dimension; the use of a take-up wheel would decrease the overall size of the fiber due to the pulling action. Moreover, an increase of the air gap could introduce an elongation stress on the fiber because of gravity. In fact, polymer chains may undergo die-swell when they exit the spinneret, but with longer air gap the swell may disappear before the nascent fiber enters the coagulation bath.

One of the limitations of our work is that urea binding on ninhydrin moieties occurs at high temperatures $\left(70^{\circ} \mathrm{C}\right)$ due to the slow binding kinetic typical of urea carbonyl sorbents [8]. For applications to WAK, one would preferably need to improve the removal without warming up the system. For this purpose, one could investigate incorporation of higher particle loading in the MMM, to use porous sorbent particles and/or to apply particles with higher ninhydrin density. Also, the optimization of the experimental conditions (i.e. higher dialysate flow rate and/or higher 
transmembrane pressure) could potentially improve particle accessibility leading to higher urea removal.

Finally, PS-Nin particles, as all the other urea-carbonyl sorbents, are not selective towards urea and can bind other nucleophilic solutes present in the spent dialysate (such as creatinine and amino acids) [8]. In order to study the potential binding competition between urea and other nucleophilic solutes to the ninhydrin moieties one should perform experiments with spent dialysate, preferably collected from clinics after patients' HD sections. A possible way to avoid binding competition there for the PS-Nin particles would be the insertion of an additional AC sorbent column [8] or AC based MMM upstream in the dialysate regeneration system to remove first the nucleophilic molecules besides urea (as, for example, creatinine).

In Chapter 6 we described the development of a new PCN HF for the removal of ammonium ions. This HF is meant to retain the ammonium ions which derive from the hydrolysis of urea by means of urease, is based on polyetherimide (PEI), as membrane forming polymer, ad polyethyleneimine (pei), as positively-charged crosslinker. We produced this PCN HF in one step by implementing the "Chemistry in the Spinneret" method, already described by Kopec et al. [29]. In fact, the majority of membrane forming polymers are negatively charged and, usually, multiple steps are needed for the addition of the positive charges to the membranes, as for example for layer-by-layer-deposition [30, 31] and thin-film-composite membrane manufacturing [32, 33]. Our PCN HF was able to retain 50\% of ammonium ions from aqueous solution after 30 minutes. After 1.5 hours, retention decreases to $30 \%$. This decline in retention could be due to the shielding of the membrane charge by the acetate counterions which concentrate in the lumen of the fiber, reducing Donnan exclusion and increasing the passage of ammonium ions.

Future works should focus on increasing the membrane permeance (now it is quite low $=0.03 \mathrm{~L} /\left(\mathrm{m}^{2}\right.$. $\mathrm{h} \cdot$ Bar)) while maintaining high retention of ammonium ions. To achieve this, one could reduce the 
wall thickness by increasing the bore solution flow rate, reducing the dope flow rate and using a takeup pulling wheel. Moreover, another strategy would be the addition of a non-solvent in the dope solution, as for example ethanol. It has been reported that small additions of a non-solvent, as ethanol, in the dope solution would delay the phase separation, leading to the reduction of pore size and increased pore density at the lumen layer as well as to the formation of a spongier structure along the membrane wall.

Here, ammonium ions retention experiments have been performed using a solution of ammonium acetate in water. To better mimic real application, further studies should be performed using: a) A urea solution which would first pass through a urease-immobilised column, to hydrolyse urea in ammonium ions; b) Dialysate spiked with urea to see the effects of other components of the dialysate (especially salts), which may shield the positive charge on the lumen layer; c) Spent dialysate from patients to investigate the potential competition effect of other toxins in the dialysate. Finally, in this work we mainly investigated one of the developed PCN HF for the removal of ammonium ions from aqueous solution although several other PCN HF with higher transport properties have been produced too (clean water permeance ranging from 7 to $108 \mathrm{~L} /\left(\mathrm{m}^{2} \cdot \mathrm{h} \cdot\right.$ Bar $)$ ). Further characterization of those fibers, as assessing the lumen surface charge and the transport properties and, based on these, other biomedical applications could be considered. Applications of these PCN HF may include removal of negatively-charged inflammatory cytokines and lipopolysaccharide adsorption as well as used as heparin binding for low blood clotting membranes.

\section{General outlook}

Overall, we believe that the results described in this thesis can pave the way towards better blood purification therapies. The AC/PES/PVP MMM offers improved blood uremic toxins removal and both MMM and OIF HF could contribute to a more continuous blood purification therapy, which will 
improve patient's health. For the first time we showed the simultaneous removal of PBTs from blood plasma and endotoxins from dialysate with the MMM. This is a very important achievement as it may open the possibility of having one membrane for blood and dialysate purification. PS-Nin MMM and the PCN HF, thanks to their ability to remove urea (in the first case) and ammonium ions (in the second case), could also contribute to the development of systems for dialysate regeneration, which are crucial for the clinical implementation of PAK and WAK.

The application of continuous therapies can reduce medical care costs, dialysis deriving waste and water consumption. In fact, 300 million dialyzers are used annually worldwide [34] which, after the HD treatment, are discarded. It is estimated that 125 billion of litres of water are consumed per year and $3.5 \mathrm{kWh}$ of electric energy are required for each treatment [35]. Considering, furthermore, that the number of patients needing HD is increasing worldwide and is expected to reach 5.5 million in 2030 [36], new considerations have to be drawn to reduce the impact of HD on the environment. Continuous therapies and dialysate regeneration systems, producing less waste and consuming significantly less water, besides improving patients' health and quality of life, will contribute to ecofriendlier HD therapies. 


\section{References}

[1] S. Eloot, D. Schneditz, T. Cornelis, W. Van Biesen, G. Glorieux, A. Dhondt, J. Kooman, R. Vanholder, Protein-bound uremic toxin profiling as a tool to optimize hemodialysis, PLoS ONE 11(1) (2016) e0147159.

[2] M.P.M. Graham-Brown, D.R. Churchward, A.C. Smith, R.J. Baines, J.O. Burton, A 4-month programme of in-centre nocturnal haemodialysis was associated with improvements in patient outcomes, Clinical Kidney Journal 8(6) (2015) 789-795.

[3] M. Dam, P.J.M. Weijs, F.J. van Ittersum, B.C. van Jaarsveld, Physical performance in patients treated with nocturnal hemodialysis - a systematic review of the evidence, BMC Nephrology 20(1) (2019) 317.

[4] T. Cornelis, S. Eloot, R. Vanholder, G. Glorieux, F.M. van der Sande, J.L. Scheijen, K.M. Leunissen, J.P. Kooman, C.G. Schalkwijk, Protein-bound uraemic toxins, dicarbonyl stress and advanced glycation end products in conventional and extended haemodialysis and haemodiafiltration, Nephrology Dialysis Transplantation 30(8) (2015) 1395-1402.

[5] A. Davenport, Portable and wearable dialysis devices for the treatment of patients with end-stage kidney failure: wishful thinking or just over the horizon?, Pediatric Nephrology 30(12) (2015) 2053-2060.

[6] A. Davenport, V. Gura, C. Ronco, M. Beizai, C. Ezon, E. Rambod, A wearable haemodialysis device for patients with end-stage renal failure: a pilot study, The Lancet 370(9604) (2007) 2005-2010.

[7] S.S. Dukhin, Y. Tabani, R. Lai, O.A. Labib, A.L. Zydney, M.E. Labib, Outside-in hemofiltration for prolonged operation without clogging, Journal of Membrane Science 464 (2014) 173-178.

[8] M.K. van Gelder, J.A.W. Jong, L. Folkertsma, Y. Guo, C. Blüchel, M.C. Verhaar, M. Odijk, C.F. Van Nostrum, W.E. Hennink, K.G.F. Gerritsen, Urea removal strategies for dialysate regeneration in a wearable artificial kidney, Biomaterials 234 (2020) 119735.

[9] D. Simazaki, M. Hirose, H. Hashimoto, S. Yamanaka, M. Takamura, J. Watanabe, M. Akiba, Occurrence and fate of endotoxin activity at drinking water purification plants and healthcare facilities in Japan, Water Research 145 (2018) 1-11.

[10] W.B. Anderson, C.I. Mayfield, D.G. Dixon, P.M. Huck, Endotoxin inactivation by selected drinking water treatment oxidants, Water Research 37(19) (2003) 4553-4560.

[11] J. Rapala, K. Lahti, L.A. Räsänen, A.L. Esala, S.I. Niemelä, K. Sivonen, Endotoxins associated with cyanobacteria and their removal during drinking water treatment, Water Research 36(10) (2002) 2627-2635. 
[12] A. Sinha, A. Bagga, Maintenance dialysis in developing countries, Pediatric Nephrology 30(2) (2015) 211-219.

[13] J. Cohen, J.L. Vincent, N.K.J. Adhikari, F.R. Machado, D.C. Angus, T. Calandra, K. Jaton, S. Giulieri, J. Delaloye, S. Opal, K. Tracey, T. van der Poll, E. Pelfrene, Sepsis: a roadmap for future research, The Lancet Infectious Diseases 15(5) (2015) 581-614.

[14] D. Kim, D. Stamatialis, High flux mixed matrix membrane with low albumin leakage for blood plasma detoxification, Journal of Membrane Science 609 (2020) 118187.

[15] H.G. B. Kraus, M. Hornung, C. Kraft, Hollow fiber membrane and method for manufacturing thereof, EP2083939B1, 2007.

[16] Z.L. Xu, F. Alsalhy Qusay, Polyethersulfone (PES) hollow fiber ultrafiltration membranes prepared by PES/non-solvent/NMP solution, Journal of Membrane Science 233(1) (2004) 101-111.

[17] J.J. Qin, J. Gu, T.S. Chung, Effect of wet and dry-jet wet spinning on the shear-induced orientation during the formation of ultrafiltration hollow fiber membranes, Journal of Membrane Science 182(1) (2001) 57-75.

[18] R.G. Gorsuch, H. Grage, H. Handley JR., H.W. Peters, J.C. Kearns, Specialized Hollow Fiber Membranes for Plasmapheresis and Ultrafiltration US 2007/0023353A1, 2007.

[19] D. Pavlenko, E. van Geffen, M.J. van Steenbergen, G. Glorieux, R. Vanholder, K.G.F. Gerritsen, D. Stamatialis, New low-flux mixed matrix membranes that offer superior removal of protein-bound toxins from human plasma, Scientific Reports 6(1) (2016) 34429.

[20] M.S.L. Tijink, M. Wester, G. Glorieux, K.G.F. Gerritsen, J. Sun, P.C. Swart, Z. Borneman, M. Wessling, R. Vanholder, J.A. Joles, D. Stamatialis, Mixed matrix hollow fiber membranes for removal of protein-bound toxins from human plasma, Biomaterials 34(32) (2013) 7819-7828.

[21] I. Geremia, R. Bansal, D. Stamatialis, In vitro assessment of mixed matrix hemodialysis membrane for achieving endotoxin-free dialysate combined with high removal of uremic toxins from human plasma, Acta Biomaterialia 90 (2019) 100-111.

[22] O.E.M. Ter Beek, New Membranes for Improved Dialysis Therapy, University of Twente, 2020.

[23] K.K.R. Tetala, D. Stamatialis, Mixed matrix membranes for efficient adsorption of copper ions from aqueous solutions, Separation and Purification Technology 104 (2013) 214-220.

[24] Y.G. Zhou, Y.D. Yang, X.M. Guo, G.R. Chen, Effect of molecular weight and degree of deacetylation of chitosan on urea adsorption properties of copper chitosan, Journal of Applied Polymer Science 89(6) (2003) 1520-1523. 
[25] A. Pathak, S.K. Bajpai, Preparation of Cu(II)-Immobilized Chitosan (CIC) and preliminary urea uptake study, Polymer-Plastics Technology and Engineering 47(9) (2008) 925-930.

[26] L.D. Wilson, C. Xue, Macromolecular sorbent materials for urea capture, Journal of Applied Polymer Science 128(1) (2013) 667-675.

[27] C. Xue, L.D. Wilson, Kinetic study on urea uptake with chitosan based sorbent materials, Carbohydrate Polymers 135 (2016) 180-186.

[28] G.W. Watt, J.D. Chrisp, Spectrophotometric method for determination of urea, Analytical Chemistry $26(3)$ (1954) 452-453.

[29] K.K. Kopeć, S.M. Dutczak, M. Wessling, D. Stamatialis, Chemistry in a spinneret-On the interplay of crosslinking and phase inversion during spinning of novel hollow fiber membranes, Journal of Membrane Science 369(1) (2011) 308-318.

[30] Z. Qin, C. Geng, H. Guo, Z. Du, G. Zhang, S. Ji, Synthesis of positively charged polyelectrolyte multilayer membranes for removal of divalent metal ions, Journal of Materials Research 28(11) (2013) 1449-1457.

[31] G. Liu, D.M. Dotzauer, M.L. Bruening, Ion-exchange membranes prepared using layer-by-layer polyelectrolyte deposition, Journal of Membrane Science 354(1) (2010) 198-205.

[32] Y. Zheng, G. Yao, Q. Cheng, S. Yu, M. Liu, C. Gao, Positively charged thin-film composite hollow fiber nanofiltration membrane for the removal of cationic dyes through submerged filtration, Desalination 328 (2013) 42-50.

[33] T.K. Dey, R.C. Bindal, S. Prabhakar, P.K. Tewari, Development, characterization and performance evaluation of positively-charged thin film-composite nanofiltration membrane containing fixed quaternary ammonium moieties, Separation Science and Technology 46(6) (2011) 933-943.

[34] C. Ronco, W.R. Clark, Haemodialysis membranes, Nature Reviews Nephrology 14(6) (2018) 394-410.

[35] C. Boccato, D. Evans, R. Lucena, J. Vienken, Water and dialysis fluids, a quality management guide, Pabst Science Publishers, 2015.

[36] T. Liyanage, T. Ninomiya, V. Jha, B. Neal, H.M. Patrice, I. Okpechi, M.H. Zhao, J. Lv, A.X. Garg, J. Knight, A. Rodgers, M. Gallagher, S. Kotwal, A. Cass, V. Perkovic, Worldwide access to treatment for endstage kidney disease: a systematic review, The Lancet 385(9981) (2015) 1975-1982. 



\section{Summary}

The patients with End Stage Kidney Disease (ESKD) waiting for or not suitable for kidney transplantation have to undergo hemodialysis (HD) therapy. The latter is a life-sustaining therapy, removing only a small fraction of uremic toxins compared to the kidney. Moreover, several drawbacks derive from the intermittent character of HD therapy (mainly 4 hours / 3 times per week in the clinic), including fluctuations in blood values and water volumes as well as poor quality of life by limiting patients' mobility and social life. Continuous or semi-continuous blood purification therapies, using portable (PAK) or wearable artificial kidney (WAK) devices could provide better therapy and significantly improve patients' health and quality of life. Importantly, the application of PAK and WAK systems will require lower amounts of dialysate $(0.5$ to $2 \mathrm{~L})$, in contrast to the current HD which requires $\sim 120 \mathrm{~L}$ per therapy.

This thesis was focused on addressing some of the above challenges which hamper the development of continuous/semi-continuous blood purification systems. More specifically, it investigated new membrane strategies for improved blood purification and dialysate regeneration. Specific attention was focused on strategies which could provide better and more continues therapy with lower water consumption, as well as strategies which could provide efficient spent dialysate regeneration.

A more detailed introduction to this thesis' topic, the scope and the outline of this thesis are presented in Chapter 1.

In Chapter 2 we investigate the application of an activated carbon (AC)/polyethersulfone (PES)/polyvinylpyrrolidone (PVP) mixed matrix membrane (MMM) for achieving for the first-time endotoxin-free dialysate and high removal of uremic toxins from human plasma with a single membrane. The MMM acts as a safety barrier to avoid the transport of pyrogens to the blood compartment and, thanks to sorbent AC, can remove approximately 10 times more endotoxins from 
dialysate compared to commercial membranes. Importantly, endotoxins from dialysate and proteinbound toxins from human plasma can be removed simultaneously without compromising $\mathrm{AC}$ adsorption capacity. We estimated that only $0.15 \mathrm{~m}^{2}$ of MMM is needed to totally remove the daily production of the PBTs, indoxyl sulfate and hippuric acid, and to completely remove endotoxins. These results could open up new possibilities for dialysis therapy with low water consumption, including PAK/WAK devices. Moreover, the MMM can have an impact where purity and scarcity of water are limiting factors for HD treatment, for which nearly $500 \mathrm{~L}$ of water are needed for the production of pyrogen-free dialysate.

Due to the direct and long-term contact with blood, one of the key factors for the clinical implementation of HD membranes, besides blood purification efficiency, is an optimal hemocompatibility profile. The hemocompatibility of the MMM, described in Chapter 2, was evaluated in Chapter 3. In the MMM, the sorbent activated carbon particles are embedded in the outer membrane layer for achieving higher removal whereas the inner blood contacting selective membrane layer is expected to achieve optimal blood compatibility. We performed ex vivo hemocompatibility experiments using freshly donated human blood and following the norm ISO 10993-4. The hemocompatibility profile of the MMM, which is characterized by low flux and no albumin leakage, is compared with a previous generation high-flux PES/PVP/AC MMM, with homemade PES/PVP single layer hollow fiber and with various control fibers already applied in the clinic. Our results show that the MMM successfully avoids contact of blood with the AC and has very good hemocompatibility, comparable to membranes currently used in the clinic.

In standard HD the blood flows in the lumen of hollow fiber hemodialyzer, while the dialysate into the inter-fiber space (IFS), in the so-called "inside-out filtration mode". In this configuration, blood clots can be formed at the inlet of the fiber thus blocking the blood flow, consequently lowering blood clearance and filter life. The "outside-in filtration" (OIF), where the blood flows into the IFS and the 
dialysate flows in the fiber lumen, could be applied to HD to prolong the operational hemodialyzer life. However, to achieve this, new HD membranes suitable for OIF, having the blood compatible selective layer at the outer surface, have to be developed. In Chapter 4 we investigated the development of hollow fibers suitable for OIF HD based on PES/PVP polymer blends. To this end, we tailored various spinning parameters, including dope and bore solutions composition, dope and bore flow rates. The resulting membranes had outer selective layer, high ultrafiltration coefficient $\left(>15 \mathrm{~mL} /\left(\mathrm{m}^{2} \cdot \mathrm{h} \cdot \mathrm{mmHg}\right)\right)$, low albumin leakage, very good creatinine clearance from human plasma as well as very good mechanical properties, matching successfully the properties of HD membranes used currently in the inside-out filtration mode.

In Chapter 5 and Chapter $\mathbf{6}$ we focused on the development of strategies for the removal of urea from spent dialysate. As a matter of fact, due to its small molecular dimension and hydrophilicity, urea removal from spent dialysate is one of the biggest challenges hampering the dialysate regeneration and the development of PAK/WAK systems. Several methods have been proposed for its removal but have not found clinical implementation due to the fact that they were not safe for the patients' health and/or not efficient enough.

In Chapter 5 we described the development of new MMM consisting of polystyrene-based ninhydrin particles, an already known carbonyl-type sorbent for urea, within a PES/PVP polymer blend matrix. Carbonyl-type sorbents had been studied earlier for urea removal in packed bed columns, with several disadvantages as high pressure drop and rather poor particles dispersion. Here, thanks to the good dispersion of small size particles within the MMM, the kinetics of urea removal is significantly higher compared to that obtained by the same particles in suspension. Importantly, in crossflow experiments, the MMM removes $3.4 \mathrm{mmol} / \mathrm{g}$ of urea from dialysate model solution, which is one of the highest amounts of urea removal reported in literature. Based on this, we could estimate that 215 grams of 
MMM would be needed to remove the daily produced urea from spent dialysate. These results are very promising for application to PAK/WAK, where miniaturization and lightweight are required.

In Chapter 6, we investigated an alternative strategy for urea removal. We developed positively charged nanofiltration (PCN) hollow fibers for the retention of ammonium ions which would derive from the hydrolysis of urea by means of urease. To fabricate these PCN hollow fiber, we implemented the 'Chemistry in the Spinneret' technique, using polyetherimide and polyethylenimine being the membrane forming material and the positively charged crosslinker, respectively. Various spinning parameters were tailored, including the bore solution composition and flow rate and the polymer dope concentration. The developed membrane had rather low water permeance, $0.03 \mathrm{~L} /\left(\mathrm{m}^{2} \cdot \mathrm{h} \cdot \mathrm{Bar}\right)$; however, the membrane is stable at least up to transmembrane pressure of 6 Bar and can retain approximately $50 \%$ of ammonium ions from the feed solution after 30 minutes.

The main conclusions of this thesis are reported in Chapter 7. Here potential directions for future developments for improved blood purification membrane strategies as well as dialysate regeneration are discussed. 


\section{Nederlandse samenvatting}

Patiënten met nierfalen (End Stage Kidney Disease, ESKD) die wachten op of ongeschikt zijn voor niertransplantatie, ondergaan hemodialyse (HD). Dit laatste is een levensreddende therapie die, in tegenstelling tot goedwerkende nieren, alleen een klein deel van de uremische toxines kan verwijderen. Sterker nog, er zijn nog een aantal nadelen op te sommen wat betreft HD, zoals het periodieke karakter van HD (voornamelijk 4 uur / 3 keer per week in de kliniek) dat leidt tot fluctuerende bloedwaarden en water volumes, maar ook een slechte kwaliteit van leven door een beperkte mobiliteit en sociaal leven. Continue of semi-continue bloedzuiveringstherapieën die gebruik maken van een draagbare, artificiële nier (PAK of WAK) kunnen een betere therapie geven en zo de kwaliteit van leven van patiënten significant verbeteren. Daarnaast is het belangrijk dat een PAK of WAK systeem maar weinig dialyse water van hoge kwaliteit nodig heeft $(0.5$ tot $2 \mathrm{~L})$, in vergelijking met $\mathrm{HD}$, waarvoor $\sim 120 \mathrm{~L}$ per therapie nodig is.

Dit proefschrift adresseert een aantal van de hierboven genoemde uitdagingen die de ontwikkeling van (semi-) continue bloedzuiveringssystemen belemmeren. Zo zijn nieuwe membraanstrategieën onderzocht voor een verbeterde bloedzuivering én dialysaat regeneratie. Er is voornamelijk aandacht besteed aan strategieën die een verbeterde en continue therapie kunnen bieden met een laag waterverbruik, maar er is ook aandacht besteed aan strategieën die voor een efficiënte dialysaat regeneratie kunnen zorgen.

Een gedetailleerde introductie in het onderwerp en een overzicht van het proefschrift staan in

\section{Hoofdstuk 1.}

In Hoofdstuk 2 onderzoeken we de toepassing van een gemengde matrix membraan (MMM) bestaande uit geactiveerde koolstof (AC), polyethersulfoon (PES) en polyvinylpyrrolidon (PVP). Voor de eerste keer beschrijven we dat een enkel membraan zowel dialysaat vrij van endotoxines 
houdt als een hoge verwijdering van uremische toxines uit humaan plasma laat zien. De MMM fungeert als een veiligheidsbarrière die het transport van pyrogenen naar het bloed belemmert en, dankzij de AC, ongeveer tien keer meer endotoxines uit het dialysaat verwijdert vergeleken met commerciële membranen. Het is belangrijk om te melden dat deze processen simultaan kunnen plaatsvinden zonder de adsorptie capaciteit van de AC te verminderen. We schatten in dat er maar $0,15 \mathrm{~m}^{2} \mathrm{MMM}$ nodig is om endotoxines én de dagelijkse productie van eiwitgebonden afvalstoffen (PBTs), zoals indoxylsulfaat en hippurisch zuur, helemaal te verwijderen. Deze resultaten brengen nieuwe mogelijkheden voor dialyse therapieën met een lage water consumptie, zoals PAK/WAK systemen. Bovendien kan de MMM een verschil maken op plekken waar een lage waterkwaliteit of waterschaarste limiterende factoren zijn van HD behandelingen, waarvoor ongeveer $500 \mathrm{~L}$ water nodig is voor de productie van pyrogeenvrij dialyse vloeistof.

Door het langdurige en directe contact met het bloed is het, naast de bloedzuiveringsefficiëntie, zeer belangrijk voor de klinische implementatie van HD membranen dat ze goed compatibel zijn met het bloed. De bloed compatibiliteit, zoals beschreven in Hoofdstuk 2, is geëvalueerd in Hoofdstuk 3. In de MMM zijn de AC deeltjes ingebed in de buitenste laag om zo hogere verwijdering van uremische toxines en endotoxines te faciliteren, terwijl de binnenste selectieve laag, die in direct contact staat met het bloed, een optimale bloed compatibiliteit laat zien. Hiervoor hebben we ex vivo bloed compatibiliteitsexperimenten uitgevoerd volgens de ISO-10993-4 richtlijnen, met vers gedoneerd humaan bloed. De bloed compatibiliteit wordt gekarakteriseerd door een lage flux en geen albumine lekkage. De resultaten zijn vervolgens vergeleken met de vorige generatie hoge flux PES/PVP/AC MMM, met een zelfgemaakte enkellaags PES/PVP holle fiber en met verschillende fibers die al in de kliniek gebruikt worden.

In standaard HD stroomt het bloed aan de binnenkant van de holle fiber en de dialysevloeistof in de ruimte tussen de fibers (IFS). Dit is de zogenaamde 'binnenste-buiten' filtratie modus. In deze 
configuratie kunnen bloedpropjes ontstaan bij de ingang van de fiber die vervolgens de bloedstroom blokkeren. Het gevolg hiervan is een lagere bloedklaring en een korter filterleven. De omgekeerde 'buitenste-binnen'(OIF) configuratie, waarbij het bloed in de IFS stroomt en de dialysevloeistof aan de binnenkant van de fiber, zou toegepast kunnen worden in HD om het filterleven te verlengen. Echter, om dit te bereiken moeten nieuwe HD membranen ontwikkeld worden met de selectieve laag aan de buitenkant, met tevens een hoge bloed compatibiliteit. In Hoofdstuk 4 hebben we met behulp van PES/PVP polymeermengsels onderzocht of we holle fibers geschikt voor OID HDkunnen produceren. Om dit te bereiken hebben we de holle fiber spinparameters, zoals de samenstelling van de polymeermengsels (de 'polymer dopes') en de samenstelling van de oplossingen die voor het lumen van de holle fiber zorgen (de 'bore solution') en de stroomsnelheden hiervan nauwkeurig onderzocht. De membranen die hieruit voortkwamen hadden de selectieve laag aan de buitenkant, een hoge ultrafiltratie coëfficiënt $\left(>15 \mathrm{~mL} /\left(\mathrm{m}^{2} \cdot \mathrm{h} \cdot \mathrm{mmHg}\right)\right)$, weinig albumine lekkage, een hele goede creatinine klaring van humaan plasma en goede mechanische eigenschappen. Hiermee hebben we dus succesvol een fiber gemaakt voor de OIF configuratie, die de eigenschappen van de huidige HD membranen (waarbij de 'binnenste-buiten' configuratie wordt toegepast) laat zien.

In Hoofdstuk 5 en Hoofdstuk 6 focussen we op de ontwikkeling van strategieën voor de verwijdering van ureum uit het dialysaat. Het is zelfs zo dat de verwijdering van ureum, dat erg klein en hydrofoob is, uit gebruikt dialysaat één van de grootste uitdagingen is voor dialysaatregeneratie en de ontwikkeling van PAK/WAK systemen. Er zijn verschillende methoden voorgesteld voor de verwijdering van ureum, maar geen van deze methoden is in de kliniek geïmplementeerd, omdat ze niet veilig waren voor de patiënten of niet efficiënt genoeg.

In Hoofdstuk 5 beschrijven we de ontwikkeling van een nieuw MMM dat bestaat uit PES/PVP met daarin kleine deeltjes van op polystyreen gebaseerde ninhydrine, dat al bekend is als een carbonyltype sorptiemiddel voor ureum. Dit soort deeltjes zijn al eerder onderzocht in gepakte kolommen voor 
de verwijdering van ureum, maar deze kolommen vertoonden verschillende nadelen, zoals een hoge drukval en een vrij slechte verdeling van de deeltjes. In onze MMM is, dankzij de goede verdeling van deze kleine deeltjes, de kinetiek van ureumverwijdering significant hoger dan wanneer dezelfde deeltjes in suspensie zitten. Belangrijker, in de experimenten waarbij tegengestelde stroomrichtingen worden gebruikt voor de vloeistof door het lumen van de holle fiber en de vloeistof buiten de holle fiber ('cross-flow experiments), verwijdert de MMM 3,4 mmol/g ureum uit het dialysaat. Dat is één van de hoogste waarden voor ureumverwijdering zoals gerapporteerd in de literatuur. Gebaseerd op deze waarde schatten we dat we 215 gram MMM nodig hebben om de dagelijkse ureumproductie uit het dialysaat te verwijderen. Dit resultaat is veelbelovend voor de ontwikkeling van PAK/WAK systemen, waarbij zowel miniaturisatie als een lichtgewicht systeem een vereiste zijn.

In Hoofdstuk 6 hebben we nog een andere strategie voor de verwijdering van ureum onderzocht. We hebben namelijk positief geladen holle fibers voor nanofiltratie (PCN) ontwikkeld voor de retentie van ammoniumionen, die afkomstig zijn van de hydrolyse van ureum door het enzym urease. Om deze PCN te maken hebben we de 'scheikunde in een spindop' techniek toegepast. Hiervoor hebben we polyetherimide als het materiaal voor het membraan gebruikt en polyethyleeninime als de geladen crosslinker. We hebben verschillende parameters aangepast, zoals de samenstelling van de 'bore oplossing', de stroomsnelheid en de concentratie van de 'polymer dope oplossing'. De ontwikkelde membranen hebben een vrij lage water doorlaatbaarheid ('permeance') van $0,03 \mathrm{~L} /\left(\mathrm{h} \mathrm{m}^{2}\right.$ bar), maar ze zijn wel stabiel bij een transmembraandruk van 6 bar en ze kunnen ongeveer $50 \%$ van de ammoniumionen van de 'feedoplossing' (de oplossing die wordt aangevoerd) vasthouden.

De belangrijkste conclusies van dit proefschrift zijn gebundeld in Hoofdstuk 7. Verder worden in dit hoofdstuk potentiële, toekomstige ontwikkelingen voor verbeterde bloedzuiveringsstrategieën en dialysaatregeneratie besproken. 



\section{List of abbreviations}

AC: Activated Carbon

ATR - FTIR: Attenuated Total Reflectance-Fourier Transform Infrared Spectroscopy

BET: Brunauer-Emmett-Teller

BSA: Bovine Serum Albumin

CCL2: C-C motif Chemokine Ligand 2

CS: Chitosan

Ct: Cycle Threshold

ESAO: European Society for Artificial Organs

ESKD: End Stage Kidney Disease

EUDIAL: European Dialysis working group

EVAL: Ethylene Vinyl Alcohol

FBS: Fetal Bovine Serum

FCGR1: high affinity immunoglobulin gamma Fc Receptor 1

GAPDH: Glyceraldehyde 3-Phosphate Dehydrogenase

GFR: Glomerular Filtration Rate

HA: Hippuric Acid

$\mathrm{Hb}$ : Hemoglobin

HD: Hemodialysis

HDF: Hemodiafiltration

HeF: Hemofiltration

HF: Hollow Fiber

HF-MMM: High-Flux Mixed Matrix Membrane

HP: Hemoperfusion

HPLC: High-Performance Liquid Chromatography

GC: Gel Content

ID: Inner Diameter

IFS: Inter-Fiber Space

IL-1 $\beta$ : Interleukin 1 beta 
IL-6: Interleukin 6

iNOS: inducible Nitric Oxide Synthase

IS: Indoxyl Sulfate

KUf: Ultrafiltration coefficients

LAL: Limulus Amebocyte Lysate

LF-MMM: Low-Flux Mixed Matrix Membrane

Lp: Water permeance

LPS: Lipopolysaccharide

MAC: Membrane Attack Complex

MCP1: Macrophage Chemotactic Protein 1

MMM: Mixed Matrix Membrane

MW: Molecular Weight

MWCO: Molecular Weight Cut-Off

NMP: N-methylpyrrolidone

OD: Outer Diameter

OIF: Outside-In Filtration

PAK: Portable Artificial Kidney

PAN: Polyacrylonitrile

PBS: Phosphate Buffer Saline

PBTs: Protein-Bound uremic Toxins

PCN: Positively Charged Nanofiltration

PEG: Polyethylene Glycol

pei: Polyethyleneimine

PEI: Polyetherimide

PES: Polyethersulfone

PLT: Platelets

PMA: Phorbol 12-Myristate 13-Acetate

PS-Nin: Polystyrene-Ninhydrin

PSu: Polysulfone

PTFE: Polytetrafluoroethylene

PVP: Polyvinylpyrrolidone 
Qb: Blood flow rate

RBC: Red Blood Cells

RRT: Renal Replacement Therapies

RT: Room Temperature

SC: Sieving Coefficient

SD: Standard Deviation

SEM: Scanning Electron Microscopy

SS: Slipskin

TAT: Thrombin-Antithrombin III-complex

TCF: Thin-Film Composite

TMP: Trans-Membrane Pressure

TNF- $\alpha$ : Tumor Necrosis Factor alpha

XPS: X-ray Photoelectron Spectroscopy

WAK: Wearable Artificial Kidney

WBC: White Blood Cells 



\section{About the author - Ilaria Geremia}

From 2019

$2015-2019$

2014

$2010-2013$

$2007-2010$

$2002-2007$
Regulatory Scientist at TEAM mastery S.r.l. (Italy)

$\mathrm{PhD}$ candidate at the University of Twente (The Netherlands)

Intern at the Johannes Gutenberg University of Mainz (Germany)

University of Trieste (Italy)

Master of Science (Medical Biotechnology, curriculum: Nanobiotechnology) Master thesis: "Development and characterization of bioactive and nanocomposite membranes based on polysaccharides" with internship at Rescoll (France)

University of Trieste (Italy)

Bachelor of Science (Biotechnology)

Bachelor thesis: "Characterization of polymeric and nanocomposite systems in biomaterials field"

Liceo XXV Aprile, Portogruaro (Italy)

High School 


\section{List of publications}

I. Geremia, M. Borgogna, A. Travan, E. Marsich, S. Paoletti and I. Donati, Determination of the Composition for Binary Mixtures of Polyanions: The Case of Mixed Solutions of Alginate and Hyaluronan, Biomacromolecules, 2014, 15(3) 1069-1073.

O. Ter Beek, I. Geremia, D. Pavlenko, D. Stamatialis, Chapter 3: Advanced Blood Purification Therapies, book: Biomedical membranes and (bio)artificial organs, World Scientific, 2017, 59-82.

I. Geremia, R. Bansal, D. Stamatialis, In vitro assessment of mixed matrix hemodialysis membrane for achieving endotoxin-free dialysate combined with high removal of uremic toxins from human plasma, Acta Biomaterialia, 2019, 90, 100-111.

I. Geremia, D. Pavlenko, K. Maksymow, M. Rüth, H.D. Lemke, D. Stamatialis, Ex vivo evaluation of the blood compatibility of mixed matrix hemodialysis membranes, Acta Biomaterialia, 2020, 111, 118-128.

I. Geremia and D. Stamatialis, Innovations in dialysis membranes for improved kidney replacement therapy, Nature Reviews Nephrology, 2020, 16, 550-551.

I. Geremia, L. Bolhuis - Versteeg, T. Kelly, M. Labib and D. Stamatialis, Development of hollow fiber hemodialysis membranes for outside-in filtration, In preparation for submission.

I. Geremia, J. A. W. Jong, C. F. van Nostrum, W. E. Hennink, K. G. F. Gerritsen, D. Stamatialis, New mixed matrix membrane for the removal of urea from dialysate solution, In preparation for submission.

Compositions for use in the simultaneous removal of endotoxins and uremic solutes during the treatment of patients; I. Geremia, D. Stamatialis; EP18185495 (2018); WO 022894 A1 (2020).

Hollow fibers for outside-in dialysis applications; M. Labib, P. Materna, I. Geremia, D. Stamatialis; US 63/034,790 (2020).

Porous membranes comprising sorbent particles for improved urea capture; I. Geremia, K.G.F. Gerritsen, C.F. van Nostrum, R. Smakman, W.E. Hennink, J.A.W. Jong, G. Yong, D. Stamatialis, et al.; EP 19211034.4 (2020). 



\section{Acknowledgments}

I spent four amazing years at the University of Twente and completing my $\mathrm{PhD}$ thesis would have not been possible without a great team and without all the people surrounding me in this journey:

Dear Prof. dr. Dimitrios Stamatialis, there are many things I need to thank you for. Thanks for giving me the opportunity of being part of your team. Dimitrios, I perfectly remember your warm welcome when I came for the interview at the University of Twente and your telephone call announcing me that I was selected for the $\mathrm{PhD}$ position. Thanks for your guidance during these years, for pushing me up when I was down and for bringing me back in the right direction when I was lost. I will always remember our scientific discussions and weekly meetings, which, being both Mediterranean, were, I would say, "animated".

My sincere gratitude goes to the committee members, Prof. dr. D.W. Grijpma, Dr. ir. W.M. de Vos, Dr. K.G.F. Gerritsen, Prof. dr. J.P. Kooman, Prof. dr. J. Van Der Vlag and Prof. dr. G. Catapano. Many thanks for having accepted to evaluate my $\mathrm{PhD}$ thesis and for participating at the defense.

The BST Department is the best group I could have hoped for. Here, the people to thank are really a lot. Dear Dirk, thanks for leading this awesome group, where I felt completely "blended" from the first day. Dear Andre, Jai and Jos, thank you for the great scientific discussions during the BST meeting and chats in the corridor. Ruchi, I really enjoyed writing a manuscript with you, thanks for all the scientific (and not) tips you gave me.

Dear Karin, what would BST be without you? I really cannot find proper words to thank you for all the support you gave me from the moment I applied for the $\mathrm{PhD}$ position to the moment of finalizing thesis printing. I knew I could always come to you for "few encouraging words" and that meant really a lot.

Lydia, thank you so much for all your precious help in the lab, arranging things and cheering us up. The spinning sessions and the experiments we performed together had always a different, more joyful, tone.

Dear Marc, Hetty and Zlata, thanks for being so active, committed and always there for all of us in the BST. When you were not there we were lost. I will never forget our nice chats!

Kasia, dear friend, thanks for everything, inside and outside the lab. You made my years at UT definitely better. Your dedication was inspiring for me. Thank you for sharing your energy and, sometimes, your stress with me!

Thijs and Odyl, my office-mates! Thanks for our conversations and support when things were against us and thanks because with you I never felt "foreigner".

Natalia and Denys, we had great time in Enschede. Thank you for the time we spent together in the lab, but also for our outings with Lia and Felix (even though you made fun of my strong Italian accent!). 
Dear Aysun, you have been my colleague for all four years and even housemate during my last months in Enschede. You care about people and I will never forget the way you took care of me during the last months. Thank you.

Dear Pia, thanks for our friendly chats and for our outings. Now, fingers crossed for our 2021 !

I confess I was very excited when Anna and Dalila joined, even though just for short periods, BST. Thanks for the cheerfulness and for our Italian evenings. I am very lucky to have you so close in Italy with Mike and Adriano. Mike, isn't it true that Italy has bewitched you??

Iris and Dooli, you joined the BAO group during my last year. Your determination and dedication gave me the last push to complete my work, thank you. And thanks for being part of the BAO Spice Girls emerging group!

Aga, Bas, Jonas, Praneeth, Marcel, Duco, Zengchao, Jia, Piet and Magda thank you for being such good buddies, for our dinners, beach-volley, movies, Sinterklaas, wine and barbecues. Hope to meet you soon again!

During my PhD I had the luck to be part of TheLink ITN Marie Curie Project. Christof and Carolyn, thank you very much for managing the project and organising all the related events. Many thanks to all my TheLink buddies, I enjoyed a lot meeting you twice per year somewhere in Europe and sharing with you our PhD progresses!

Basia, Bram, Casper, Caterina and Giorgio, you have been like a family when mine was far. Thank you!

Simone, thanks for making my thesis cover and layout definitely better.

To my family, the best in the world, many thanks!

And now you, Save. Well, we made it! There won't ever be words to express my gratitude for all your support and patience (a lot of patience). Let's move on and let's focus now on a new adventure!!! 